Claremont Colleges

Scholarship@ Claremont

All HMC Faculty Publications and Research

HMC Faculty Scholarship

$1-1-1992$

\title{
Design of Turbopump Propellant Feed Systems
}

Mary P. Cardenas

Harvey Mudd College

\section{Recommended Citation}

Cardenas, Mary P."Design of Turbopump Propellant Feed Systems," pp. 155-218 in D. Hunzel and D. Huang, Modern Engineering for Design of Liquid-Propellant Rocket Engines, American Institute of Aeronautics and Astronautics, 1992.

This Book Chapter is brought to you for free and open access by the HMC Faculty Scholarship at Scholarship @ Claremont. It has been accepted for inclusion in All HMC Faculty Publications and Research by an authorized administrator of Scholarship @ Claremont. For more information, please contact scholarship@cuc.claremont.edu. 


\section{Design of Turbopump Propellant Feed Systems}

For high-thrust, long-duration liquid-propellant rocket engines, the use of turbopump-fed systems usually lowers system weight and raises performance as compared to pressurized-gas fed systems. Turbopump-fed systems require only relatively low pumpinlet pressures, and thus propellant-tank pressures, while the major portion of the pressure required at the thrust-chamber inlets is supplied by the pumps. This saves considerable tank weight, particularly in large vehicles.

As the overall trend toward higher chamber pressure for liquid-propellant rocket engines continues, the role of turbopumps in an engine system becomes of greater importance, particularly with the high-performance hydrogen-fueled engines. The advantage of pump-fed over pressure-fed engines increases as mission velocity requirements increase, and becomes very substantial as orbit-insertion velocities are approached.

Figure 6-1 shows a representative range of pump operating parameters for various liquid-propellant rocket engine applications. Figure 6-2 depicts an envelope of power requirements for a number of actual turbine designs.

\subsection{ELEMENTS OF TURBOPUMP-FED SYSTEMS}

The vehicle designer must insure that propellants go to the inlet of the pumps at required minimum pressures. The turbopump feed system raises the pressure of the propellants received from the vehicle tanks and delivers them to the main thrust chamber, through ducts and valves, at pressures and flow rates commensurate with rated engine operation. A turbopump feed system may consist of the following basic elements:

- Propellant pumps.

- Turbine(s) to $\cdot$ drive them.

- Power source for the turbine(s) (during engine start as well as main stage).

- Speed-reduction gear transmissions (if any).

- Lubrication system for bearings and gears.

- Accessory drives.

- Propellant inlet and discharge ducts.

- Turbopump mounts.

\section{Propellant Pumps}

The principal requirements of a rocket-engine propellant pump are high reliability, low cost, light weight, stable flow for the required operating range, high efficiency, adequate suction performance, and long life. The relative importance of these factors and their resulting influence on the design will vary depending on the application. The most widely used pump types are centrifugal (or radial), axial, or mixed flow. Centrifugal pumps are usually designed with a single stage; axial pumps, multistage. However, multistage centrifugal pumps with crossover-type diffusion systems have also been used effectively. Various pump configurations are shown schematically in Fig. 6-3.

Centrifugal pumps. Almost all operational rocket propellant pumps are of this type. They can handle large flows at high pressures efficiently as well as economically in terms of weight and size. The elements of a centrifugal pump are shown in Fig. 6-4 and 6-16. Centrifugal pumps, like other steady-flow rotating machinery, consist essentially of two basic elements: the rotor and the stator. These accelerate the fluid flow by imparting kinetic energy to it in the rotor and then decelerating, or "diffusing," it in the

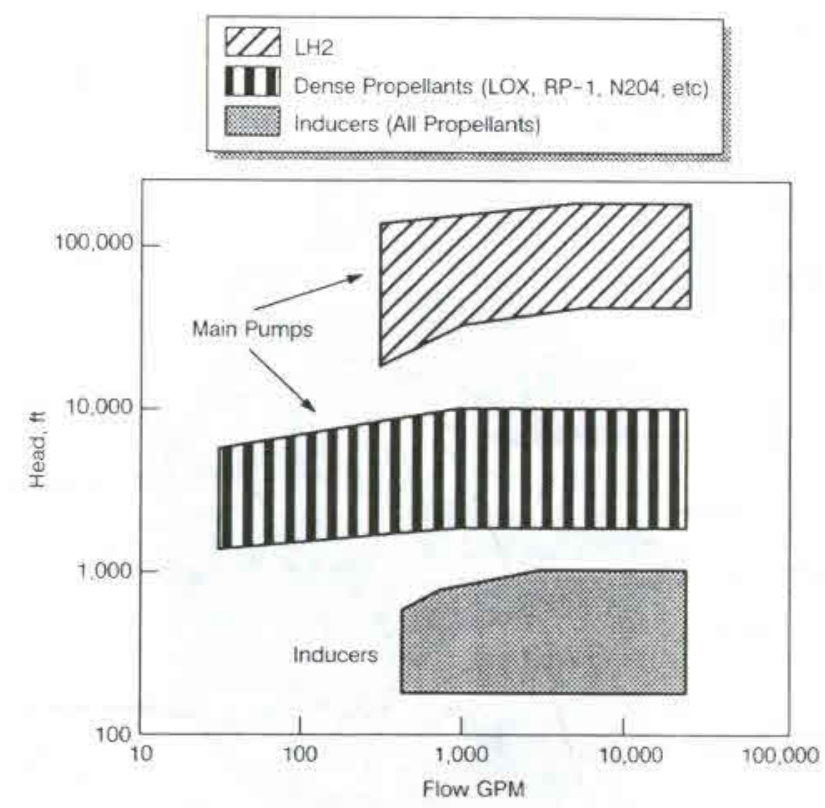

Fig. 6-1 Range of operation for typical propellant pumps.

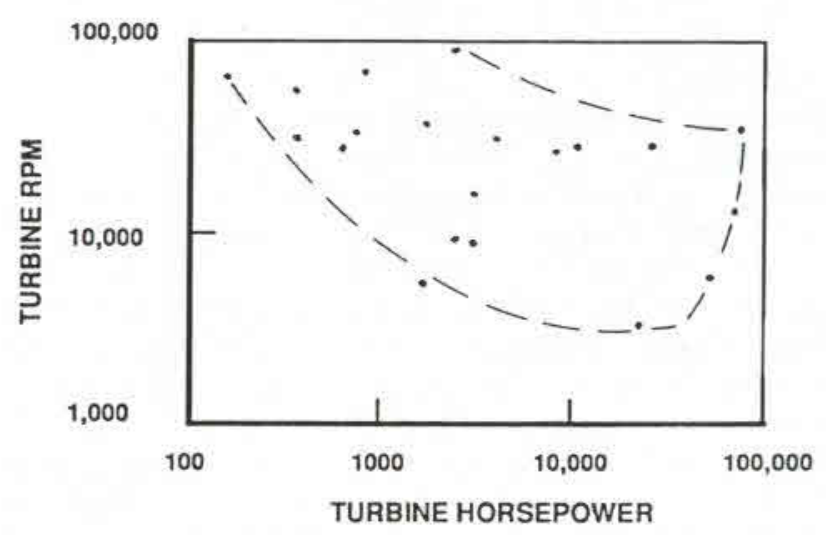

Fig. 6-2 Rocket-engine turbine design envelopes. 


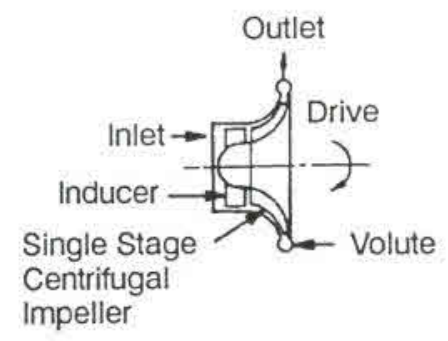

Single Stage Centrifugal Pump

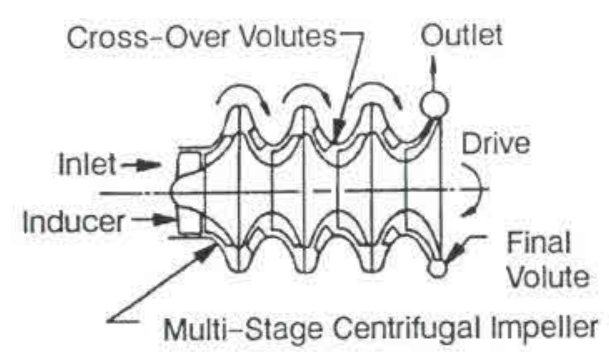

Multi-Stage Centrifugal Pump

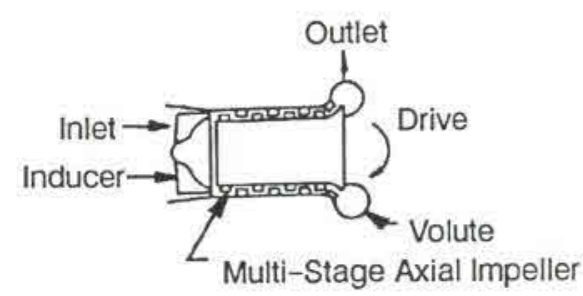

Multi-Stage Axial Pump
Fig. 6-3 Pump configurations.

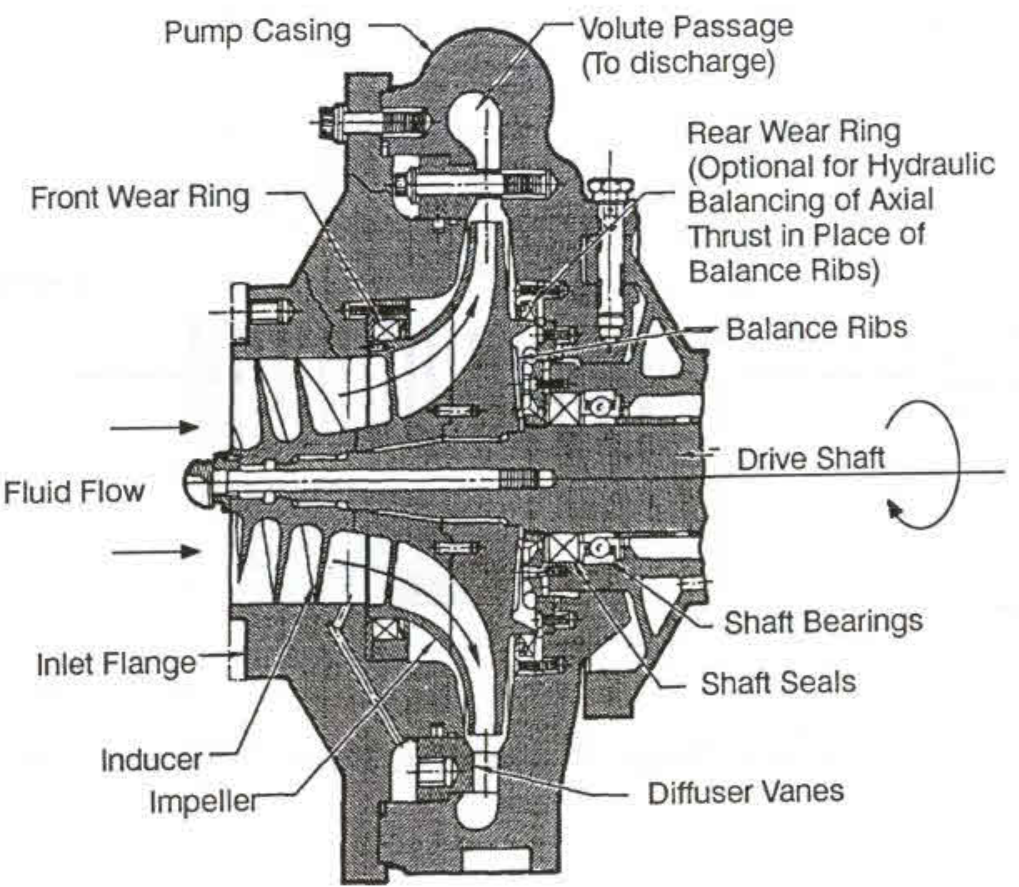

Fig. 6-4 Elements of a centrifugal-flow pump. stator. This results in increased fluid pressure head, The rotor assembly usually includes an inducer, an impeller, bearings, and a shaft. The stator assembly consists of a casing with stationary diffuser vanes, a volute with discharge outlet, and seals.

An inducer, an axial-flow rotor, increases total pressure of the entering fluid sufficiently to permit noncavitating operation of the main impeller. An inducer can reduce the pump-inlet-pressure net positive suction head (NPSH) requirements substantially. The impeller of a centrifugal (or radial) pump basically is a rotating wheel with blades that discharge the flow in a radial direction. Fluid is admitted axially to the impeller that, when rotating in an enclosure, ejects it at the periphery with increased absolute velocity (Fig. 6-4).
As primary functions, the pump stator assembly diffuses (i.e., decelerates) the fluid to convert the velocity head into pressure head, collecting and redirecting the fluid to the pump discharge outlet, and provides structural support and a pressure enclosure for the pump. Wear rings (see Fig. 6-4) provide axial-thrust control and minimize internal leakage-circulation of the fluid between the highpressure (discharge) and the low-pressure (inlet or suction) zones. Dynamic shaft seals prevent external leakage along the shaft.

Multistage centrifugal pumps. For higher pressure rises, multiplestage centrifugal pumps can be designed if a single stage proves limited. A multistage pump basically resembles a single-stage pump, except that it requires proper channeling of 
the fluid between stages. Figure 6-5 shows such a design used on the Space Shuttle Main Engine (SSME) high-pressure fuel turbopump (HPFTP).

Multistage axial pumps. This design permits achieving high head through multiple staging with smaller-diameter pumps. It has been used for liquidhydrogen pump applications. It is not as well suited for wide-flow-range operation, as will be discussed below, but can be more efficient in applications requiring relatively high ratios of flow vs. head (or at high "specific speeds," a term to be defined below). Elements of an axial-flow pump are shown in Fig. 6-6. The rotor assembly consists of an inducer, a cylindrical rotor with multiple rows of rotating blades, and a rotor shaft. The stator assembly includes a cylindrical casing with rows of stationary blades spaced between inducer and rotating blades, a volute casing, bearings, and seals.

An inducer placed at the pump inlet supplies the fluid to the main-pump section at the required pressure and velocity. Both rotor and stator blades have a hydrofoil shape. The rotor blades accelerate the flow relative to the stator and thus increase the kinetic energy of the fluid, while the stator blades, acting as diffusers, convert the velocity head of the fluid into pressure head. However, the velocity vector of the fluid in the axial direction is kept essentially constant throughout the various stages of the pump.

Inducer pumps. The simplest axial-flow pump consists of a single rotor designed as an inducer to achieve good suction, as shown in Fig. 6-7. The inducer can consist of either a single- or doubleblade row, an example of the latter being shown in Fig. 6-8. These pumps are used as low-speed "boost pumps" to raise the pressure sufficiently to permit the main pump to operate at much higher speeds to reduce its size and cost. The inducer design for this pump is similar to those used in either centrifugalor axial-flow pumps.

\section{Turbines}

The turbines (see Fig. 6-9 and 6-16) that provide shaft power to the propellant pumps typically derive their energy from the expansion of a high-pressure, hightemperature gas to lower pressures and temperatures. Turbines can be divided into two major types: impulse and reaction. Impulse turbines can be either single- or multiple-stage. Reaction turbines are usually multistage. Impulse turbines are most frequently used for high-pressure-ratio, low-flow applications. Reaction-bladed turbines are more frequently used for low-pressure-ratio, high-flow designs.

Single-stage, single-rotor impulse turbine. This turbine consists of a single-rotor disk or turbine wheel to which is attached a row of turbine blades or buckets. Gas is fed to the rotating blades through stationary nozzles (Fig, 6-10), In the nozzles, the gas pressure is converted into kinetic energy (velocity head) with attendant static-pressure drop. The gas flow reaches maximum velocity upon entering the rotating blades, where the kinetic energy of the gas is imparted to the turbine rotor as mechanical energy of rotation. Ideally, the static pressure of the gas remains constant when it passes through the rotating blades (except for the effects of friction).

Velocity-compounded impulse turbine. In this type of turbine, as shown schematically in Fig. 611, two separate rows of rotating blades instead of one transfer the kinetic energy of the gas discharged from the set of stationary nozzles. A row of stationary blades between the wheels guides the gas into the

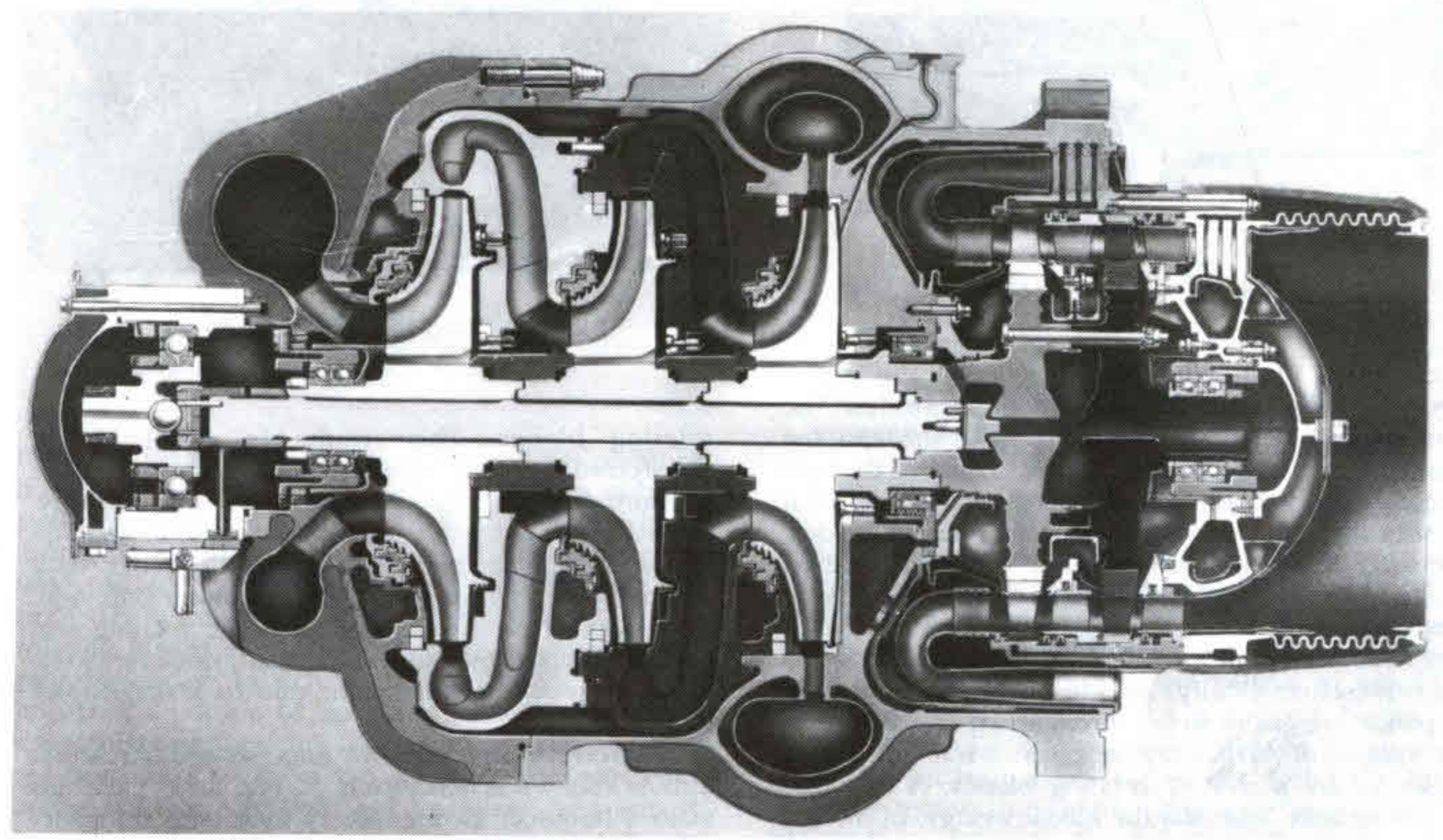

Fig. 6-5 SSME HPFTP. 


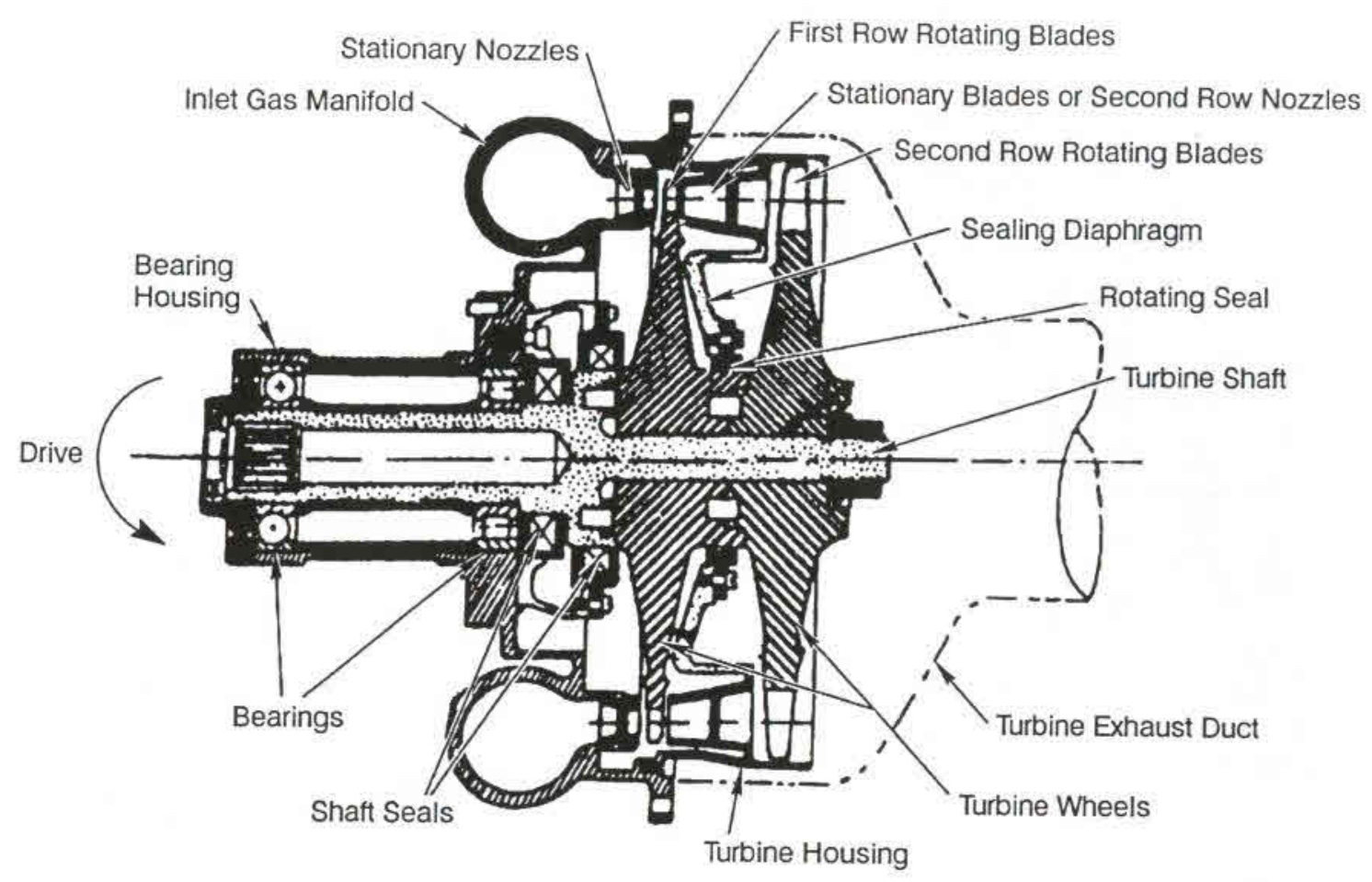

Fig. 6-9 Turbine elements.

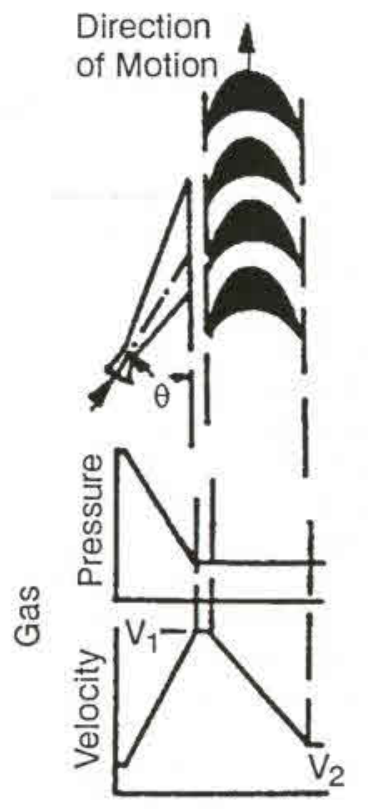

Fig. 6-10 Single-stage, single-rotor impulse turbine.

through the rotating blades, whereas the pressure does drop (expansion occurs) in a reaction turbine. Both impulse and reaction wheels are driven by a change in momentum of the gas. In a pure reactiontype turbine, the driving force is derived entirely from the reaction due to gas expansion within the rotating blades (similar to the gas expansion in a rocket nozzle). In actual reaction-turbine designs, however, a portion of the driving force comes from gas impingement on the rotating blades. The percent reaction is the ratio of the static-pressure decrease across the rotor divided by the pressure drop across both the nozzle and rotor expressed as a percentage. Thus, a $50 \%$-reaction design has equal pressure drops

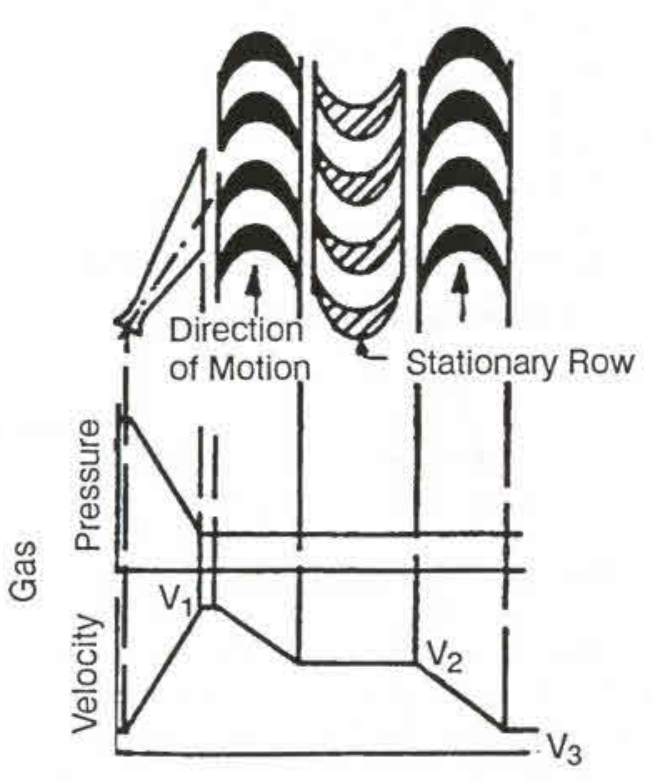

Fig. 6-11 Single-stage, two-rotor, velocitycompounded impulse turbine.

across the rotor and the nozzle. Any magnitude of reaction can be designed, but typically values of $50 \%$ or less are more efficient.

Hydraulic turbines. Occasionally it will be more economical in the engine system to use the high-pressure liquid from the pump discharge to drive a hydraulic turbine which derives its energy from the pressure drop across the turbine rather than the temperature of the driving gaseous fluids. The turbine shown in Fig. 6-7 does this to simplify sealing requirements by using liquid oxygen as the 


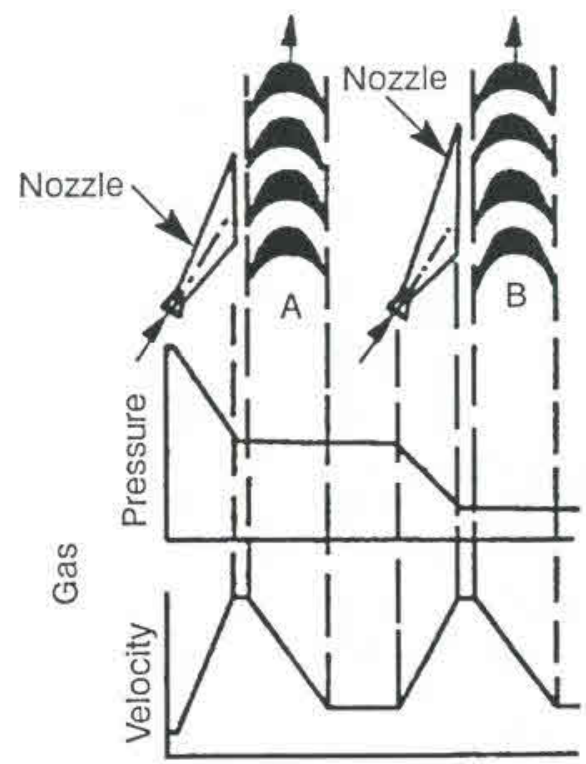

Fig. 6-12 Two-stage, pressure-compounded impulse turbine.

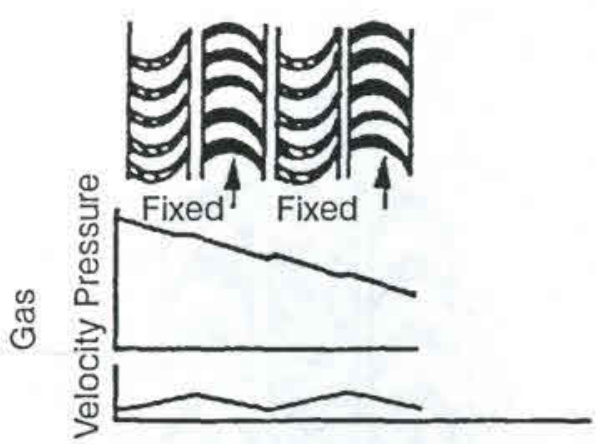

Fig. 6-13 Reaction turbine.

drive fluid in the turbine as well as in the pump. Such turbines are typically of the reaction type.

\section{Turbine Power Sources}

Several different types of engine cycle are available and can be classified primarily based on where the turbine-drive fluid originates and where it is discharged after leaving the turbine. The typical turbine power sources shown in Fig. 6-14 are described briefly here.

Bipropellant gas generator. This was the most widely used system before 1970 since it has the advantage of using the engine main propellants, and the turbine circuit is in parallel with the thrust chamber flow. The turbine-drive fluid consists of hot gas from the combustion process in the gas generator. The turbine flow is subsequently dumped overboard or to some low-pressure point. This system obviously operates with a high pressure ratio and low flowrate to minimize impact on engine specific impulse. The turbine for this system will typically be an impulse supersonic design.

Monopropellant gas generator. This provides the simplest gas-generating system, but it
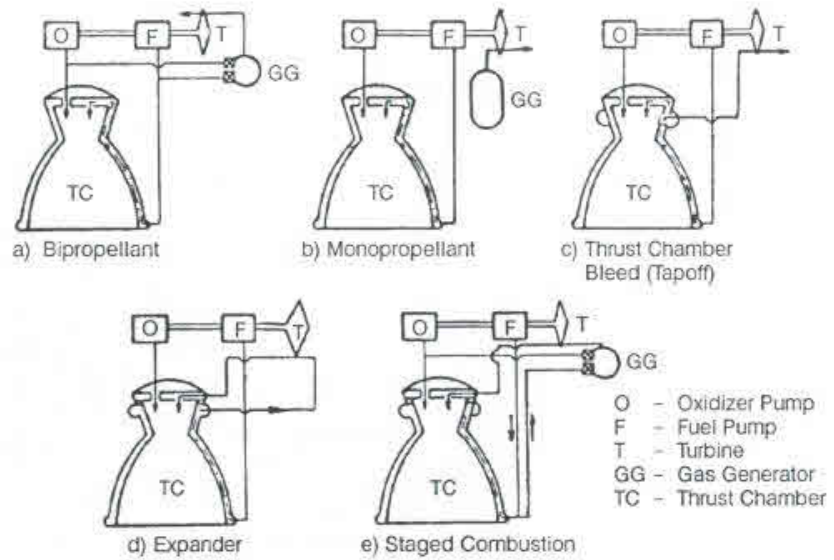

Fig. 6-14 Typical turbine power sources.

requires a third propellant if neither one of the main propellants is suitable for monopropellant application.

Thrust-chamber bleed. As applied in "tapoff" engine systems, gases will be bled off directly from the main combustion chamber to drive the turbine. The gas has an elevated temperature from the combustion process in the main chamber. Since the turbine-drive fluid is dumped overboard after leaving the turbine, this type of design is similar to those used for the gas-generator system.

Expander. In an expander cycle, such as with a hydrogen-fueled engine system, the heated hydrogen gas emerging from the thrust-chamber cooling jacket is used as the turbine working fluid before being injected into the main combustion chamber. In systems using a mono-propellant as one of the main propellants, the monopropellant can be decomposed and used to drive the turbine prior to injection into the main combustion chamber.

Dual (staged) combustion. Here the entire fuel flow reacts with a portion of the oxidizer in a bipropropellant gas generator and thus provides the gas to drive the turbine. The usually fuel-rich exhaust gas is then ducted into the main combustion chamber and reacts with the balance of the oxidizer. The turbine-discharge flow goes to the thrust chamber, resulting in a similar turbine design as the expander cycle, but at higher temperature. Stagedcombustion-cycle efficiency equals that of the expander cycle. The SSME engine is of this type. A variation of this cycle is to use an oxidizer-rich gas turbine for the oxidizer pump and a fuel rich turbine for the fuel pump.

Most systems require an auxiliary power source during engine start to drive the turbine until the main power source takes over. Evaluating start methods on a new engine usually calls for comparison between a spin and a tank-head start. A spin start uses an auxiliary power source to provide the initial buildup of turbine speed. A tank-head start uses propellants out of the main tanks for initial turbine power. As the speed increases, pump-discharge pressures increase, and the turbine flow and available power increase, resulting in the engine bootstrapping itself to mainstage. The primary fac- 


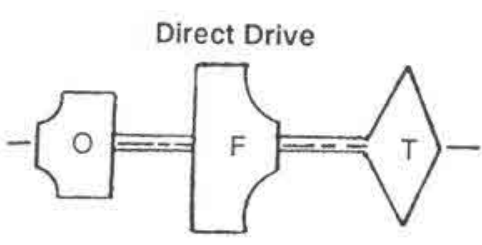

A. Pumps Back-To-Back

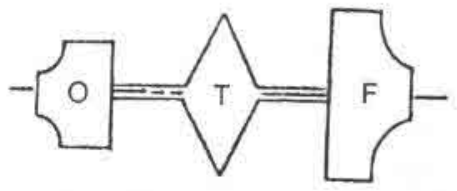

B. Turbine Between Pumps
B. Off-Set Turbine
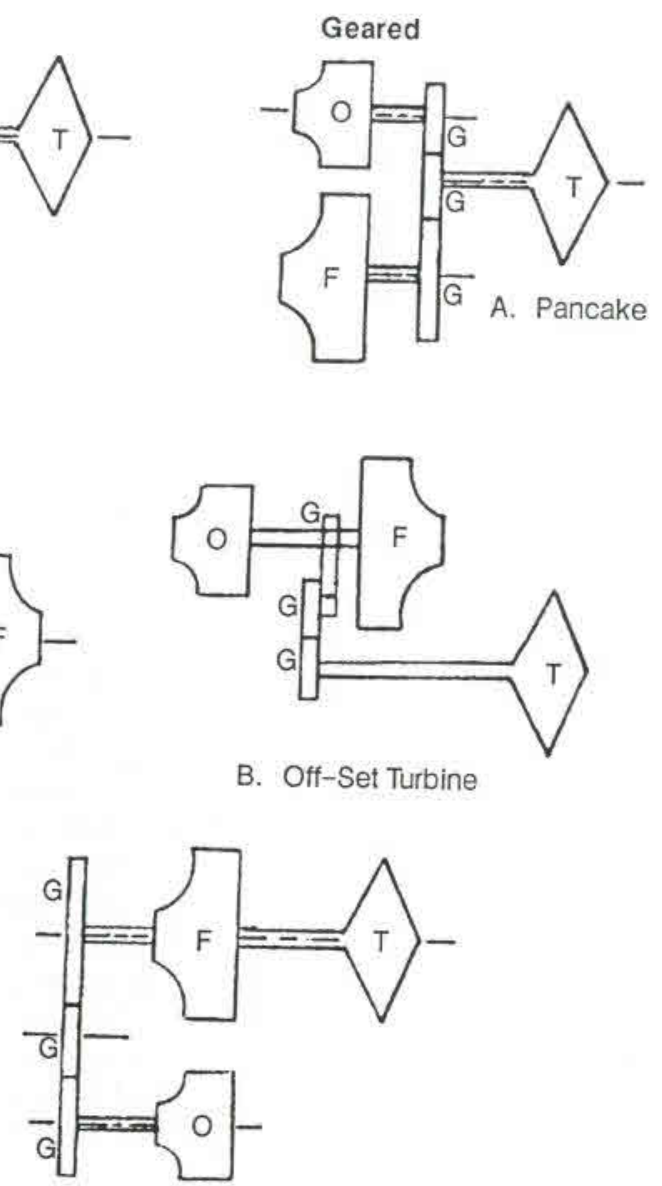

C. Single-Geared Pump

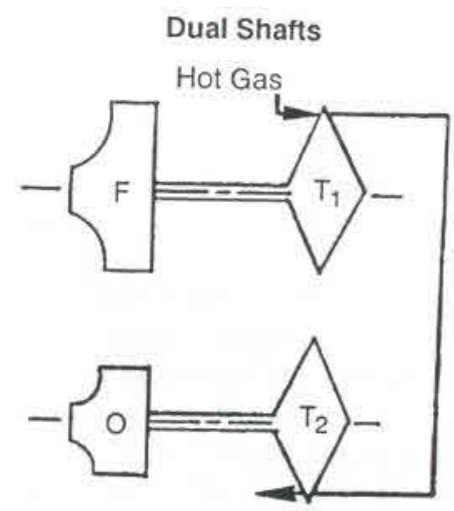

A. Turbines in Series

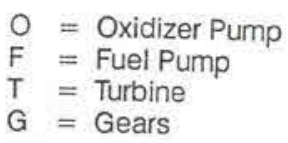

$T=$ Turbine

$\mathrm{G}=$ Gears

Fig. 6-15 Principal turbopump drives.

tors affecting the start method are the type of fuel used and the engine cycle. Whether the oxidizer is cryogenic or storable is not a major factor.

Storable fuel. A storable fuel will be a liquid at ambient temperature and pressure, such as RP-1 (kerosene) and the hydrazines. Since storables do not gasify at low pressures, relatively high initial flowrates can be obtained under tank-head conditions. This makes a tank-head start a reasonable option. Spin starts have also been used where stored high-pressure gas, a solid-propellant gas generator, or bipropellant start tanks generate the initial turbine power. Using a spin start will usually require less development but at the expense of the additional components.

Cryogenic-fuel; gas-generator cycle. All cryogenic pump-fed engines have used hydrogen for the fuel. The following discussion concerns using hydrogen, but would also apply for other cryogenic fuels. At mainstage, a gas-generator cycle tends to have a high turbine pressure-ratio. For a tank-head start, the initial turbine pressure-ratio will be lower than mainstage because the initial pressure driving the turbine has to be lower than the engine fuel-inlet pressure. This results in a relatively low initial power and a slow rate of bootstrap. Also, because hydrogen vaporizes easily at low pressure, it is difficult to get enough hydrogen flow to prevent temperature spikes in the gas generator. This makes a tank-head start very difficult. The gas-generator cycle does lend itself fairly easily to a spin start because only a small portion of the total hydrogen goes to the gas generator. During the spin portion of start, the main fuel valve will be open, providing a path for the main fuel flow to prevent stalling of the main fuel pump. Initial combustion for a spin start will be at a significantly higher pressure than a tank-head start, and will therefore reduce the likelihood of turbineinlet temperature spikes. Thus, a spin start will usually be recommended for hydrogen-fueled engines with a gas generator.

Cryogenic fuel; expander cycle. An expandercycle engine has a relatively low turbine pressureratio at mainstage because the turbine flow goes into the main combustion chamber. During start, prior to main-chamber prime, there is very low back-pressure. As a result, a tank-head start will be a turbine pressure-ratio equal to or higher than mainstage. This produces a high turbine power for a relatively rapid speed-buildup. Also, the expander does not have any combustion upstream of the turbines, so temperature spikes are not a problem. This cycle should therefore be fairly easy to tank-head start. It is also difficult to spin start because a significant 
portion of the fuel-pump flow goes through the turbine. Therefore, a high turbine-inlet pressure generated by a spin system restricts the fuel-pump flow, resulting in a low flow-coefficient. Because a hydrogen pump is very susceptible to stall and boilout at low flow, this cycle should use a tank-head start.

Cryogenic fuel; staged-combustion engine. In the staged-combustion engine, most of the fuel flow is used by the turbines; therefore it has the same potential fuel-pump stall problem (for a spin start) as the expander cycle. The tank-head start has the difficult problem of preventing turbine-inlet temperature spikes at low flowrates. Even though difficult, tank-head start is used for the staged-combustion cycle, but start temperature spikes can lead to turbine-blade damage. They can force use of coatings for thermal protection.

\section{Turbopump-Drive Arrangements}

The specific type of coupling between turbine and pumps depends not only upon the propellants being pumped but also on the design of the overall engine system. Various turbopump-drive arrangements are shown schematically in Fig. 6-15. Where a single turbine directly drives both propellant pumps through a common shaft, it can be located either on the shaft end (with back-to-back pump arrangement) or between pumps. Then both pumps and turbine will operate at the same shaft speed. Gear-driven turbopump arrangements include the pancake type, which uses different reduction gears and is applied where there are speed differentials between pumps and turbine; the offset turbine, with both pumps on one shaft but driven through a gear train; and the single-geared pump where one pump is mounted with the turbine on the same shaft, while the other is driven through a reduction gear. Dual-shaft turbopump arrangements with pump and turbine for each propellant on separate shafts include two gas turbines in series, with the discharge gas from the first turbine driving the second turbine, and two gas turbines in parallel, both receiving gas directly from the power source.

\section{Description of Developed Turbopump Systems}

Figure 6-16 shows the major elements of a typical geared liquid-bipropellant turbopump system for a rocket engine, Developed for a 188,000 -lb-thrust LOX/RP-1 booster engine, this dual-pump unit consists of oxidizer pump, fuel pump, reduction gearbox, accessory drive adapter, and a turbine. The turbine is started by hot gases from a turbine spinner (solid-propellant gas generator) and powered from a liquid-propellant gas generator during mainstage. The turbine shaft drives a series of reduction gears, which in turn drive the pump shaft. The turbopump gears and bearings are cooled and lubricated either by a separate oil-supply system or by a fuel-additive subsystem. During mainstage operation, the turbopump supplies oxidizer and fuel to the main thrust chamber as well as to the gas generator at the required pressures and flow rates. Table 6-1 lists operating characteristics and materials of construction for this turbopump.

Both oxidizer and fuel pumps are of single-entry, centrifugal-flow type. They are mounted back-to-back on a common shaft, one on each side of the gearbox. The fuel pump is bolted to the gearbox,

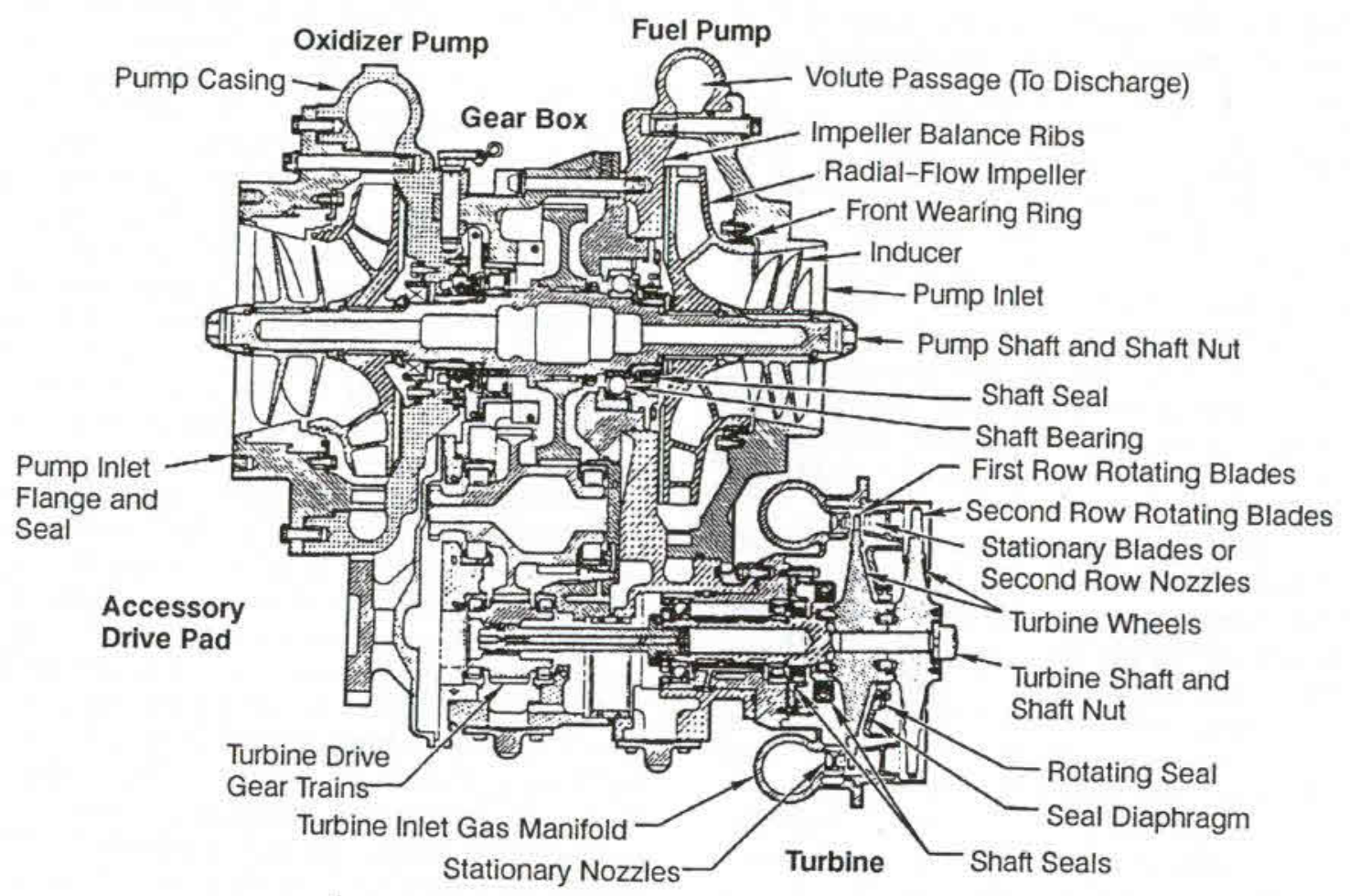

Fig. 6-16 Major elements of a geared turbopump. 
Table 6-1 Operating characteristics and construction materials for turbopump shown in Fig. 6-16.

\begin{tabular}{|c|c|c|}
\hline \multirow[b]{2}{*}{ Pumps: } & Oxidizer & Fuel \\
\hline & & \\
\hline Fluid & Liquid oxygen & RP-1 \\
\hline Inlet density & $71.38 \mathrm{lb} / \mathrm{t}^{3}$ & $50.45 \mathrm{lb} / \mathrm{ft}^{3}$ \\
\hline Inlet pressure (total) & 80.0 psia & 57.0 psia \\
\hline Discharge density & $70.95 \mathrm{lb} / \mathrm{ft}^{3}$ & $50.55 \mathrm{lb} / \mathrm{tt}^{3}$ \\
\hline Discharge pressure (total) & 915.2 psia & $1,023.0$ psia \\
\hline Pressure rise in pump & $835.2 \mathrm{psi}$ & $966.0 \mathrm{psi}$ \\
\hline Pump developed head & $1,696.2 \mathrm{ft}$ & $2,751.0 \mathrm{ft}$ \\
\hline Volume flow & $3,257.4 \mathrm{gpm}$ & $2,007.6 \mathrm{gpm}$ \\
\hline Flow rate & $518.0 \mathrm{lb} / \mathrm{s}$ & $225.7 \mathrm{lb} / \mathrm{s}$ \\
\hline Shaft speed & \multicolumn{2}{|c|}{$6537 \mathrm{rpm}$} \\
\hline Efficiency & $75.5 \%$ & $72.1 \%$ \\
\hline Shaft power & 2,117 bhp & 1,585 bhp \\
\hline NPSH required & $35.0 \mathrm{ft}$ & $35.0 \mathrm{ft}$ \\
\hline Casing material & \multicolumn{2}{|c|}{ TENS 50-T6 aluminum alloy sand casting } \\
\hline Inducer material & 7075-T6 aluminum alloy die forging & 2024-T351 aluminum alloy plate \\
\hline Impeller material & TENS 50-T6 aluminum alloy sand casting & $9669-48230-3$ aluminum alloy sand casting \\
\hline Shaft material & \multicolumn{2}{|c|}{4340 alloy steel } \\
\hline Bearing material & \multicolumn{2}{|c|}{9310 alloy steel } \\
\hline \multicolumn{3}{|l|}{ Turbine: } \\
\hline Inlet gas pressure (total) & \multicolumn{2}{|c|}{597.6 psia } \\
\hline Exit gas pressure (static) & \multicolumn{2}{|c|}{32.86 psia } \\
\hline Pressure ratio: Total inlet/static exhaust & \multicolumn{2}{|c|}{18.21} \\
\hline Inlet gas pressure (static) & \multicolumn{2}{|c|}{517.8 psia } \\
\hline Inlet gas temperature & \multicolumn{2}{|c|}{$1200^{\circ} \mathrm{F}$} \\
\hline Exit gas temperature & \multicolumn{2}{|c|}{$938^{\circ} \mathrm{F}$} \\
\hline Gas flow rate & \multicolumn{2}{|c|}{$17.34 \mathrm{lb} / \mathrm{s}$} \\
\hline Brake horsepower & \multicolumn{2}{|c|}{$3,793 \mathrm{hp}$} \\
\hline Shaft speed & \multicolumn{2}{|c|}{$31,740 \mathrm{rpm}$} \\
\hline Efficiency & \multicolumn{2}{|c|}{$66.2 \%$} \\
\hline Housing material & \multicolumn{2}{|c|}{ Hastelloy "B" } \\
\hline Nozzle block material & \multicolumn{2}{|c|}{ Hastelloy "B" } \\
\hline Wheel material & \multicolumn{2}{|c|}{ Timken alloy 16-25-6 AMS-5727 steel } \\
\hline Shaft material & \multicolumn{2}{|c|}{4340 alloy steel } \\
\hline Bearing material & \multirow{2}{*}{\multicolumn{2}{|c|}{9310 alloy steel }} \\
\hline Gearbox: & & \\
\hline Reduction speed ratio & \multicolumn{2}{|c|}{$1 / 4.855$} \\
\hline Gearbox material & \multicolumn{2}{|c|}{ TENS 50-T6 aluminum alloy sand casting } \\
\hline Gear and shaft material & \multicolumn{2}{|c|}{9310 alloy steel die forging } \\
\hline Bearing material & \multicolumn{2}{|c|}{9310 alloy steel } \\
\hline
\end{tabular}


while the oxidizer pump is secured to it by radially inserted steel pins. These pins allow the oxidizerpump housing to expand and contract during extreme temperature changes without distortion and misalignment. Each pump has an axial-flow inducer, a radial-flow impeller with backward curved vanes, stationary diffuser vanes, and a volute. The diffuser vanes and volute are designed to achieve uniform pressure at the outer diameter of the impeller to minimize radial loads. Balance ribs on the back side of the impellers neutralize pump-shaft axial thrust.

The gearbox includes a series of full-depth reduction spur gears with integral-bearing inner races, gear carrier and main-shaft bearings, accessory drives, pump-shaft bearing seals, and a bearing heater on the oxidizer-pump shaft. A drain manifold handles horizontal drainage. The gears reduce the speed of rotation between turbine and pump shaft by an overall ratio of 4.88 to 1 . Details of typical turbopump gears and bearings are shown in Fig. 6-17. The pump shaft turns clockwise as viewed from the oxidizer pump. The sequence of power transmission goes from turbine to high-speed pinion gear then and to pump-shaft gear. Power is also transmitted to a main accessory-drive gear from a drive pinion gear mounted on the intermediate gear shaft.

The turbine-an impulse-type, two-stage pressure-compounded unit (Fig. 6-12) bolted to the fuelpump housing - consists of a hot-gas inlet manifold, stationary nozzles and vanes, first- and second-stage turbine wheels, a turbine shaft, and a splined quill shaft connecting the turbine shaft to the high-speed pinion gear. The turbine shaft is supported on the inboard side by a ball bearing; on the outboard side, by a roller bearing. Carbon-ring shaft seals prevent hot-gas leaks. The turbine-inlet manifold distributes the gases to the first row of stationary nozzles. They, in turn, distribute the gases to the first row of rotating blades. When leaving these, the gases again increase velocity when passing through the second row of stationary nozzles. They finally pass through the second row of rotating blades and leave the turbine through an exhaust duct. A sealing diaphragm between the first and second turbine wheel prevents the hot gas from bypassing the second row of stationary nozzles.

Figure 6-18 shows a direct-drive liquid-oxygen turbopump for a dual-shaft turbopump system. The main pump element, a double-suction design, has a rotor consisting of mirror-image inducers and backto-back centrifugal impellers. The flow splits equally in the inlet, flows through the inducers and impellers, and is rejoined at the impeller outer diameter, subsequently flowing through the vaned diffuser and volute. As can be seen in Table 6-2, this pump has a discharge pressure at rated power level of 4100 psi, but $9.3 \%$ of the oxygen flow is tapped off downstream of the main pump and sent through the small centrifugal "kick" pump, which raises the pressure to $6952 \mathrm{psi}$. The shaft system rotates on duplex ball bearings, which are cooled by a positive flow of the liquid oxygen from the pump.

The turbine-drive gas comes from a preburner system, the turbopump being used on a stagedcombustion-cycle engine. The turbine, a two-stage axial-flow reaction design, has a small reaction rate to help minimize axial loads. A mixture of liquid hydrogen and hot gas cools the turbine disks and housing to attain structural integrity. The turbinedrive fluid is prevented from mixing with the liquid oxygen by a complex seal package consisting of a main LOX seal, double-floating-ring intermediate seal with an interior helium purge, and a double-

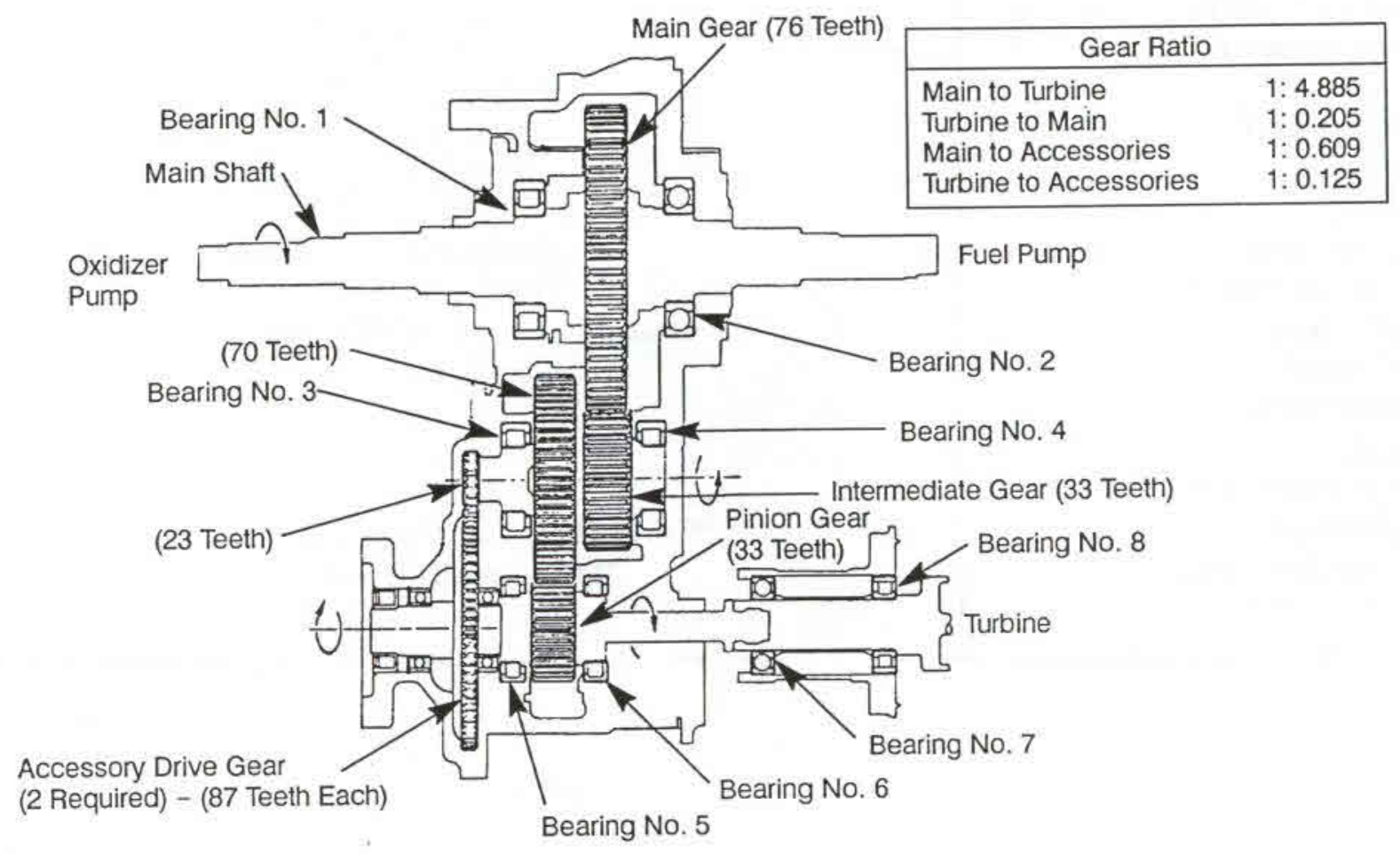

Fig. 6-17 Typical turbopump gears and bearings. 


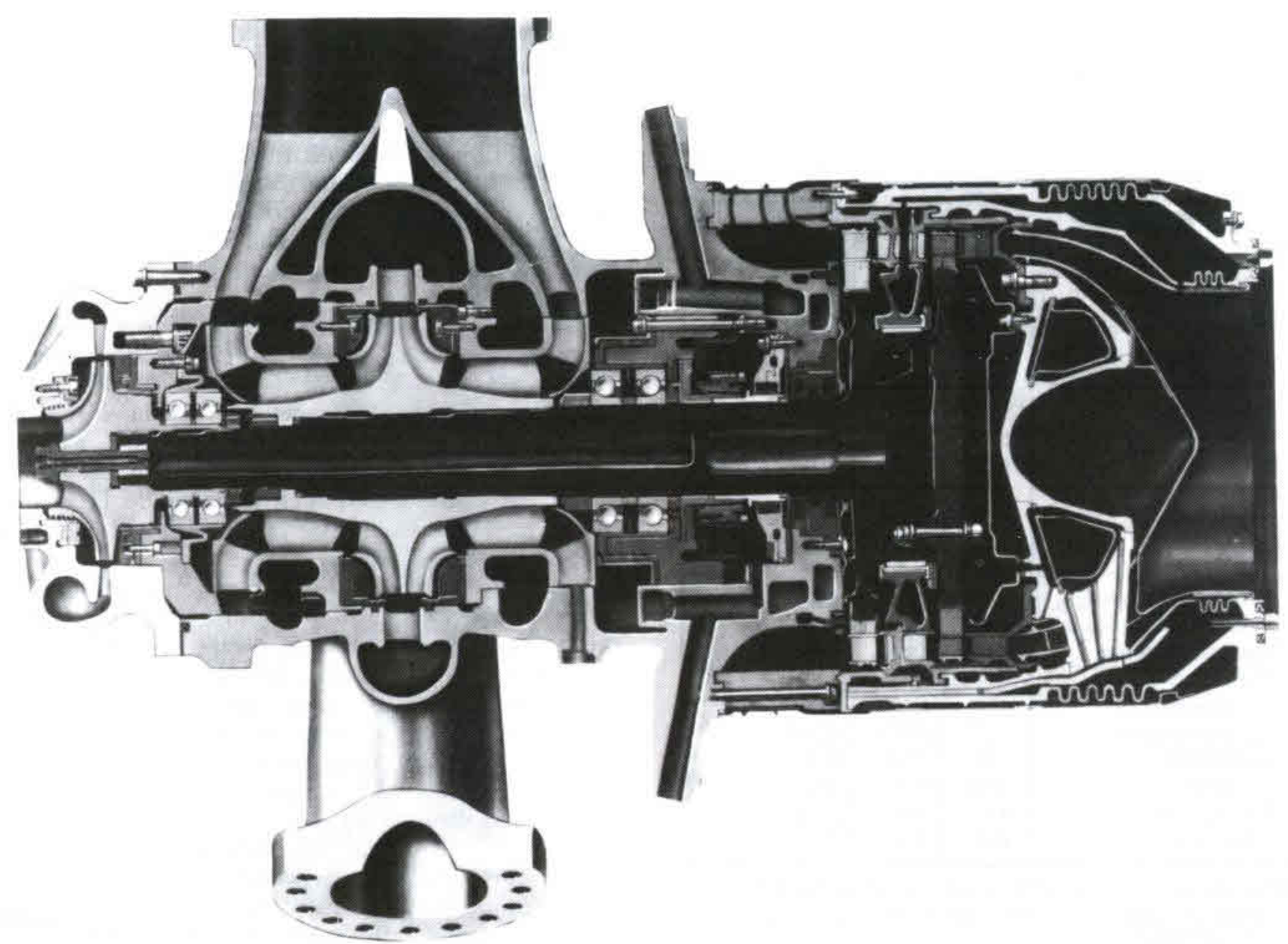

Fig. 6-18 SSME HPOTP.

floating-ring hot-gas seal. Overboard drains minimize the required helium-supply flowrate.

Axial thrust is controlled by a double-acting balance piston located on the front shrouds of the back-to-back main impeller.

\subsection{TURBOPUMP SYSTEM PERFORMANCE AND DESIGN PARAMETERS}

Being closely related, variation of the turbopump design and operating parameters will contribute to the optimization of both turbopump and engine system performance. The design procedure for the turbopump system includes evaluation of all possible design approaches and mechanical configurations that can satisfy engine system specifications. From this will be selected the best design with respect to overall systems reliability and performance. Further discussion of this subject can be found in Ref. 6-1.

\section{Turbopump System Performance}

Turbopump performance affects the vehicle payload in three ways:

1) Turbopump component weight. Since the turbopump components form part of stage burnout weight, they directly affect stage payload.
2) Required pump-inlet suction pressure. Required suction pressure directly translates into required main-propellant-tank pressure level. Suction pressure raised, tank and pressurization-system weights increase and thus reduce the stage payload for a given burnout weight.

3) Turbine-gas flowrate. For gas-generator cycles, the turbine-drive gases are usually ejected at a lower specific impulse than the thrust-chamber gases. Their flowrate decreases the overall $I_{S}$ of the engine system and thus for a given velocity increment it decreases the allowable stage-burnout weight. For a fixed weight of engines, tanks, guidance, and other equipment, a decrease in allowable stage-burnout weight decreases payload weight.

\section{Turbopump System Design Parameters}

In the design of turbopump systems, the following parameters, often interdependent, are considered paramount: propellant properties, pump-developed heads and flowrates, pump specific speeds, pump net positive suction head (NPSH), pump efficiencies, overall performance and operating efficiency of turbine, turbopump-system cycle efficiency, and turbopump-system calibration and off-design characteristics. No simple rules are available for optimizing the correlations of these parameters when designing a specific type of turbopump for a given engine 
Table 6-2 Operating parameters for turbopumps on the SSME at rated power level.

\begin{tabular}{|c|c|c|c|c|}
\hline \multirow[b]{2}{*}{$\begin{array}{l}\text { Turbopump } \\
\text { Figure Reference }\end{array}$} & \multirow[b]{2}{*}{$\begin{array}{l}\text { LPOTP } \\
6.7\end{array}$} & \multirow[b]{2}{*}{$\begin{array}{c}\text { HPFTP } \\
6-5\end{array}$} & \multicolumn{2}{|c|}{ HPOTP } \\
\hline & & & $\begin{array}{l}\text { Main } \\
6.18\end{array}$ & Boost \\
\hline \multicolumn{5}{|l|}{ Pump: } \\
\hline Fluid & LOX & $\mathrm{LH}_{2}$ & LOX & LOX \\
\hline Inlet density, $\mathrm{lb} / \mathrm{ft}^{3}$ & 71.03 & 4.32 & 70.28 & 70.28 \\
\hline Total inlet pressure, psia & 100.0 & 250 & 374.8 & 4,031 \\
\hline Total discharge pressure, psia & 408.8 & $6,024.8$ & $4,129.8$ & $6,952.2$ \\
\hline Pump developed head, $\mathrm{ft}$ & 625.9 & 168,920 & 7,591 & 5,934 \\
\hline Flow rate, lb/s & 890.3 & 148.5 & 1,067 & 101.2 \\
\hline Volumetric flow at inlet, gpm & 5,626 & 15,436 & 6,814 & 594.7 \\
\hline Shatt speed, rpm & 4,961 & 33,974 & 27,039 & 27,039 \\
\hline Efficiency, \% & 68.6 & 77.3 & 67.3 & 82.5 \\
\hline Shaft power, bhp & 1,476 & 58,970 & 21,882 & 1,330 \\
\hline \multicolumn{5}{|l|}{ Turbinea: } \\
\hline Fluid & LOX & Hot gas & Hot gas & \\
\hline Inlet total pressure, psia & 3.961 & 4,933 & 4,924 & \\
\hline Discharge total pressure, psia & 408.8 & 3,376 & 3,286 & \\
\hline Isentropic velocity ratio & 0.465 & 0.356 & 0.286 & \\
\hline Pressure ratio, T-T & .. & 1.461 & 1.498 & \\
\hline Inlet lemperature, 'R & 191.3 & 1835.9 & 1430 & \\
\hline Discharge temperature, 'R & 189.7 & 1698.3 & 1314.9 & \\
\hline Flow rate, lb/s & 176.9 & 145.6 & 60.6 & \\
\hline Horsepower & 1,476 & 58,972 & 23,212 & \\
\hline Shaft speed, rpm & 4,961 & 33,974 & 27,039 & \\
\hline Efficiency, \% & 63.1 & 79.6 & 78.1 & \\
\hline
\end{tabular}

a The turbine for the LPOTP is a hydraulic turbine, and the two high-pressure turbines are two-stage pressure compounded designs using $\mathrm{O}_{2}-\mathrm{LH}_{2}$ combustion products. systems application. Generally speaking, however, available pump suction pressure together with the basic pump flow characteristics will determine the maximum shaft speed at which the unit can operate. The higher this shaft speed, the lower the turbopump weight will likely be. Once the pump speed is determined, turbine type, turbine driving arrangement, and turbine power source will be selected on the basis of efficiency, weight, simplicity, and other factors.

Propellant properties. General data for some propellants used in liquid-rocket engines are given in Tables 1-3 to 1-5. Table 6-3 lists properties of commonly used liquid propellants that have specific significance in the design of pumps. These propellants include earth-storable liquids such as RP1 and $\mathrm{N}_{2} \mathrm{O}_{4}$, cryogenics such as $\mathrm{LO}_{2}$ and $\mathrm{LH}_{2}$, and liquids having a wide range of physical and chemical properties.

The low temperature of cryogenic liquids imposes design limitations that must be addressed in the selection of materials, seals, bearings, and lubricants. For example, the temperature is low enough to freeze any water vapor present, creating ice that can damage parts or cause them to malfunction. The total temperature range to which the structural elements of a cryogenic turbopump may be exposed varies from -300 to $-430^{\circ} \mathrm{F}$ at the pumps to 1200 to $1700^{\circ} \mathrm{F}$ at the turbine. This induces temperature gradients between the various turbopump components that must be accommodated, and requires structural flexibility or suitable devices to

Table 6-3 Fluid properties of commonly used liquid propellants.

\begin{tabular}{|c|c|c|c|c|c|c|}
\hline \multirow[b]{3}{*}{ Liquid } & \multicolumn{6}{|c|}{${ }^{a}$ Data at Normal Conditions } \\
\hline & \multirow[b]{2}{*}{$\begin{array}{l}\text { Temper- } \\
\text { ature, } \\
\cdot F\end{array}$} & \multirow[b]{2}{*}{$\begin{array}{c}\text { Vapor } \\
\text { Pressure, } \\
\text { psia }\end{array}$} & \multirow[b]{2}{*}{$\begin{array}{l}\text { Density } \\
\mathrm{Ib} / \mathrm{f}^{3}\end{array}$} & \multicolumn{2}{|c|}{ Conversion Factors } & \multirow[b]{2}{*}{$\begin{array}{l}\text { Viscosity, } \\
\text { |b-s/in? }\end{array}$} \\
\hline & & & & $\begin{array}{c}\text { Head ft/ } \\
\text { Pressure, } \\
\text { psi }\end{array}$ & $\underset{\mathrm{lb} \cdot \mathrm{s}}{\mathrm{gpm} /}$ & \\
\hline $\mathrm{N}_{2} \mathrm{O}_{4}$ & 60 & 11.1 & 90.7 & 1.59 & 4.96 & $0.637 \times 10^{-7}$ \\
\hline $\mathrm{H}_{2} \mathrm{O}_{2}(90 \%)$ & 60 & 0.026 & 87.8 & 1.64 & 5.12 & $1.868 \times 10^{-7}$ \\
\hline $\mathrm{N}_{2} \mathrm{H}_{4}$ & 60 & 0.158 & 63.3 & 2.28 & 7.12 & $1.49 \times 10^{-7}$ \\
\hline \multirow{2}{*}{$R P-1$} & 60 & 0.031 & 50.8 (max) & $2.83(\mathrm{~min})$ & 8.84 (min) & $3.22 \times 10^{-7}$ \\
\hline & & & 49.8 (min) & 2.89 (max) & 9.00 (max) & \\
\hline Ethyl-alcohol (95\%) & 60 & 0.062 & 50.4 & 2.85 & 8.91 & $2.22 \times 10^{-7}$ \\
\hline UDMH & 60 & 1.83 & 49.66 & 2.90 & 9.06 & $0.842 \times 10^{-7}$ \\
\hline $50 \%$ UDMH/N ${ }_{2} \mathrm{H}_{4}$ & 60 & 1.77 & 56.66 & 2.54 & 7.94 & $1.378 \times 10^{-7}$ \\
\hline $\mathrm{LO}_{2}$ & -297.6 & 14.7 & 71.17 & 2.02 & 6.32 & $0.2765 \times 10^{-7}$ \\
\hline $\mathrm{LF}_{2}$ & -307 & 14.7 & 94.21 & 1.54 & 4.79 & $0.353 \times 10^{-7}$ \\
\hline $\mathrm{CLH}_{2}^{2}$ & -422.9 & 14.7 & 4.43 & 32.5 & 101.5 & $0.0208 \times 10^{-7}$ \\
\hline $\mathrm{bLN}_{2}$ & -320.4 & 14.7 & 50.44 & 2.86 & 8.92 & $0.226 \times 10^{-7}$ \\
\hline$b_{\text {water }}$ & 60 & 0.256 & 62.37 & 2.31 & 7.2 & $1.64 \times 10^{-7}$ \\
\hline
\end{tabular}

\footnotetext{
${ }_{b}^{a}$ Normal conditions do not necessarily imply standard conditions, if tank pressures have been applied.

$b$ Included here because these fluids are frequently used as pump calibration media.

${ }^{c}$ Conversion factors are affected by thermodynamic effects for large pressure changes.
} 
permit the required thermal expansion and contraction. Radial connecting pins are often employed to permit a cryogenic pump to contract independently of a turbine and of a normal-temperature pump.

The vapor pressure of the propellants under normal engine operation conditions directly influences total suction-pressure requirements at the pump inlet. This will be further discussed in conjunction with the pump-inlet net positive suction head (NPSH).

The density variations of different propellants produce substantially different pump headrise requirements, as well as large differences in volume flow. The power required per unit weight flow and pressure rise of a pump is inversely proportional to the density of the fluid-dramatically evident in the case of liquid hydrogen, which has a density of less than $10 \%$ of that of other propellants. For the same weight flow and pressure rise, a liquid-hydrogen pump requires more than 10 times the volume flow and driving power of other propellant pumps. Thus the design of liquid-hydrogen pumps requires specific considerations.

The viscosity of the pumped fluids effects a boundary layer along the surface of the flow passages within the pump. There is a minimum size of the impeller cross-section below which pump performance will fall off rapidly. As the passage clearances in the impeller are decreased, a point will be reached where the flow is predominantly boundary layer, and viscous forces rather than turbulent forces become predominant. This completely changes the performance of a pump. The drag effect of the boundary layers, together with the rotation of the fluid in certain pump passages (such as in the impeller of a centrifugal pump), results in secondary flows being set up. These flows, together with friction losses (also in direct proportion to the viscosity of the fluid) and leakage losses, constitute a major portion of the energy losses in a pump. A high propellant viscosity tends to lower pump efficiency.

Some liquid propellants, such as $\mathrm{LF}_{2}$ and $\mathrm{N}_{2} \mathrm{H}_{4}$, are highly reactive chemically and are thermally unstable beyond certain temperature limits. In the design of turbopumps for these propellants, special consideration must be given to the selection of compatible materials as well as to the construction of mechanical parts. Seals, bearings, and the protection (insulation) of the pump section against heat influx from the turbine section following engine shutdown are typical problem areas.

Pump-developed beads and flow rates. The pump adds energy to the fluid preferably in the form of higher discharge static-pressure. The term "pumpdeveloped head" (DH), defined as the difference between pump-discharge total head and pump suction head, represents the energy added per pound of pumped fluid expressed as a change in enthalpy with units of $\mathrm{ft}-\mathrm{lb} / \mathrm{lb}$, which is typically shortened to units of "ft." For an incompressible fluid, developed fluid pressure $[\Delta \mathrm{p}(\mathrm{psi})]$ and fluid head. $[\Delta \mathrm{H}(\mathrm{ft})]$ have this relationship:

$$
\Delta \mathrm{H}=144 \Delta \mathrm{p} / \text { (fluid density) }
$$

where $\Delta p$ is in units of psi and density is in units of $\mathrm{lb} / \mathrm{ft}^{3}$. For a fluid like hydrogen that has a degree of compressibility, the head is typically defined as the isentropic enthalpy rise from inlet conditions to the discharge pressure. The specific head/pressure relationship is dependent on the fluid-thermodynamic state conditions. For small pressure changes (e.g., less than $1000 \mathrm{psi}$ ), the incompressible relationship shown above can generally be used as a first approximation.

The required pump-developed head at the design propellant flowrate (i.e., engine thrust level) is dictated by the sum of the hydraulic resistances within the engine's propellant-flow system. These resistances include the pressure drops across injector, thrust-chamber manifold, cooling jacket, propellant valves and ducts, as well as the thrustchamber pressure. In addition, for the staged combustion cycle and the expander-cycle engine, pressure differential across the turbine must be provided for in the pump-discharge pressure. Figure 6-19 shows an engine propellant-flow-system hydraulic-resistance curve representing the resistance head to flowrate relationship at various pump operating levels.

The head is developed by the rotating members imparting energy to the fluid primarily in the form of kinetic energy. This kinetic energy is then converted by the stationary members into pressure energy. The magnitude of the developed head for a given design is a function of both the pump speed and flow. The head is generally expressed by a head-flow (H-Q) characteristic at one or more constant values of speed, as shown in Fig. 6-19. At high flows, the pump-drive energy is consumed by internal flow losses, and the head rise decreases to zero. At the other extreme, as flow decreases the head continues to increase, at least at first. It can then level off, begin slowly to decrease, or, in some cases, drop off dramatically due to phenomena known as stall. That unstable condition, discussed below, must be avoided to achieve constant pump

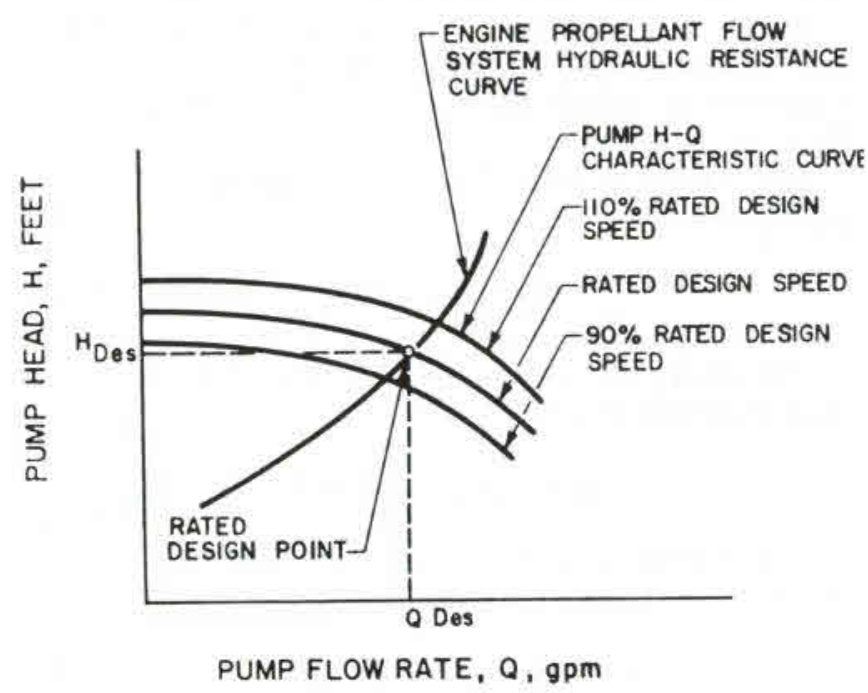

Fig. 6-19 Engine system resistance and pump characteristics. 
operation. Increasing speed increases the head by the speed-squared relationship provided the ratio of flow/speed can be maintained constant. This is strictly true only for an incompressible fluid, but approximately valid even for a compressible liquid like hydrogen.

Two dimensionless coefficients are frequently used to indicate the head and flow characteristics of a given pump: pump head coefficient $\psi$ and pump flow coefficient $\phi$. The pump head coefficient is the ratio of rated pump head ( $\mathrm{ft}$ ) to the maximum theoretical head at zero flow for meridional (axial) inlet (no prerotation), expressed as follows:

$$
\psi=\Delta \mathrm{H} /\left(\mathrm{U}_{2}{ }^{2} / g\right)
$$

where-

$\psi=$ overall pump head coefficient at rated design point (range is 0.4 to 0.7 for a singlestage centrifugal pump and 0.2 to 0.4 for a single-stage axial pump)

$\Delta \mathrm{H}=$ pump rated developed head, $\mathrm{ft}$

$\mathrm{U}_{2}=$ mean tip velocity of pump impeller at rated design rotating speed, $\mathrm{ft} / \mathrm{s}$

$g=$ gravitational constant, $32.2 \mathrm{ft} / \mathrm{s}^{2}$

The pump flow coefficient $\phi$ can be defined based either on inlet or discharge conditions, each as a ratio of the average meridional velocity $(\mathrm{cm})$ in $\mathrm{ft} / \mathrm{s}$ divided by a tip speed $\mathrm{U}$ in $\mathrm{ft} / \mathrm{s}$ (that is, $\phi=\mathrm{cm} / \mathrm{U}$ ). The inlet flow coefficient uses inlet velocity based on the flow and the geometric area from the first rotorinlet tip to the hub. The value of $\mathrm{U}$ is based on the inducer tip diameter, if present, or the impeller eye diameter (tip diameter at the inlet of the blade). For the discharge flow coefficient, the average meridional velocity at the impeller discharge is used together with the tip speed at the impeller exit. Inlet flow coefficients for rocket-engine pumps typically range from 0.07 to 0.30 . Discharge flow coefficients range from 0.01 to 0.15 .

Pump specific speeds. For any given pump design, the relation between fluid flow rate $Q$, developed fluid head $\Delta H$, required driving power $h p$, and rotating speed $\mathrm{N}$ can be defined by three expressions called the affinity laws of a pump. These laws state: speed:

1) Pump volume flowrate varies directly with

$$
\mathrm{Q}_{1} / \mathrm{Q}_{2}=\mathrm{N}_{1} / \mathrm{N}_{2}
$$

2) Pump-developed head varies directly as the square of the speed:

$$
\Delta \mathrm{H}_{1} / \Delta \mathrm{H}_{2}=\mathrm{N}_{1}^{2} / \mathrm{N}_{2}^{2}
$$

3) Pump driving power varies directly as a cube of the speed:

$$
\mathrm{hp} 1 / \mathrm{hp} 2=\mathrm{N}_{1} 3 / \mathrm{N}_{2} 3
$$

The affinity laws are based on the assumption that the pump efficiency is independent of the rotating speed. Actual pump operation has shown, however, that pump efficiency does change with speed. The degree depends on the individual pump design and the pumped fluid. Most rocket-engine propellants are basically incompressible and show less than 2 or $3 \%$ change in efficiency over a wide speed range. Thus the pump affinity laws hold quite well in most cases. Because much more strongly affected by speed, hydrogen pumps need efficiency calculated by a thermodynamic model of the pump system at the desired speeds.

The relationships established in Eq. (6-3a), (6$3 b)$, and (6-3c) permit deriving a useful pump-design parameter, the pump stage specific speed, NS:

$$
\mathrm{N}_{\mathrm{S}}=\mathrm{N}(\mathrm{Q})^{0.5 /(\Delta H)^{0.75}}
$$

$$
\begin{aligned}
\text { where- } & \\
\mathrm{N}_{\mathrm{S}} & =\text { pump specific speed } \\
\mathrm{N} & =\text { pump rotating speed, rpm } \\
\mathrm{Q} & =\text { pump flowrate, gpm } \\
\Delta \mathrm{H} & =\text { pump-developed head in a given stage }
\end{aligned}
$$

Pump specific speed is a characteristic value typically defined at the point of maximum efficiency, which is usually the design point. In pump design, this term is very useful to classify inducers or impellers on the basis of both performance and geometric proportions regardless of the actual size or speed at which they operate. $N_{S}$, a function of design configuration, does not vary significantly for a series of geometrically similar impellers (having the same angles and proportions), or for a particular impeller operating at any speed.

Figure 6-20 indicates typical pump specific speeds for various impeller geometries. For a given speed, a low value of specific speed is characteristic for low-volume flow, high-headrise pumps. Higher specific speed indicates a higher-volume-flow, lowhead-rise pump.

Radial-type impeller. The head is largely developed by action of centrifugal force. This radial type is used for specific-speed from 500 to 1200 . Geometric proportion $\left(\mathrm{r}_{2} / \mathrm{r}_{1}\right)$ varies from 2 to 3 .

Francis-type impeller. This type has an axial inlet and a radial discharge, and is used for lower heads. Specific speed generally ranges from 1000 to 2400. Geometric proportion $\left(r_{2} / r_{1}\right)$ varies from 1.3 to 1.8. The Francis type is most representative of rocket-engine pumps.

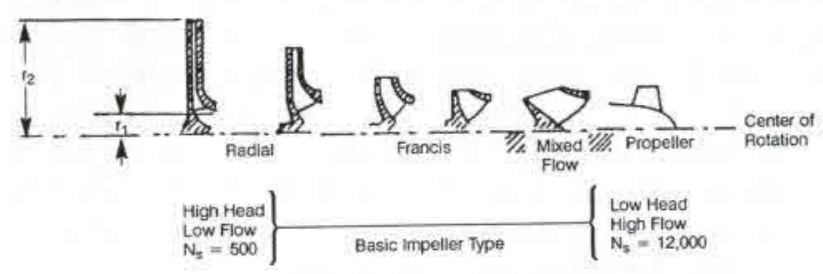

Fig. 6-20 Relationship between the pump specific speeds and pump-impeller geometries. 
Mixed-flow-type impeller. The head developed in this impeller is due partly to change in tangential velocity and partly to change in fluid velocity relative to the rotor. Discharge is partly radial and partly axial. The impeller blades are doubly curved. Specific speed varies from 2200 to 3500.

Propeller-type impeller. The head developed by this type is through push of the vanes only. Flow direction is axial. Specific speeds range from 3000 to 6000 for multi-stage impellers, and from 6000 to 12,000 for inducers.

\section{Sample Calculation 6-1}

The following data characterize the propellant pumps of the hypothetical A-1 Stage engine at the rated design point:

\section{Pump}

Fluid density, $\mathrm{lb} / \mathrm{ft} 3$

Inlet total pressure, psia

Pump discharge pressure, psia

Pump flowrate, $\mathrm{lb} / \mathrm{s}$

Pump speed, rpm

$\begin{array}{cl}\text { Oxidizer } & \text { Fuel } \\ 71.38 & 50.45 \\ 55 & 45 \\ 1505 & 1720 \\ 1971 & 892 \\ 7000 & 7000\end{array}$

\section{Problem}

Determine the pump specific speeds.

\section{Solution 6-1}

For the oxidizer pump: developed pressure developed head $=1450 \mathrm{psi}$

$=2930 \mathrm{ft}$ volumetric flowrate $\quad=12,420 \mathrm{gpm}$ specific speed $\quad=1980$

For the fuel pump:

developed pressure $\quad=1675 \mathrm{psi}$

developed head $\quad=4790 \mathrm{ft}$

volumetric flowrate $\quad=7960 \mathrm{gpm}$

specific speed $\quad=1083$

Pump net positive suction bead (NPSH); cavitation. Allowing the static pressure of the fluid at the pump inlet or any regions within the pump to drop below the local fluid vapor-pressure level will cause these regions to cavitate; i.e., the fluid will pass from liquid to vapor and form bubbles. The formation of vapor alters the effective flow passages of the fluid and can seriously affect normal pump performance. The subsequent collapse of these vapor regions creates local pressure forces that can cause flow instabilities and/or substantial damage.

To avoid cavitation during operation of a propellant pump, the pump-inlet available net positive suction head (NPSH) a furnished by the propellant-feed system upstream of the pump, must be higher than the suction head above the propellant vapor pressure at which cavitation would begin. (NPSH) $)_{a}$ is the difference between the propellant inlet total pressure head and the propellant vapor pressure, expressed as follows:

$$
(\mathrm{NPSH})_{\mathrm{a}}=(144 / \rho)\left[\mathrm{P}_{\mathrm{t}}-\Delta \mathrm{pf}-\mathrm{p}_{\mathrm{v}}\right]+\mathrm{Z}
$$

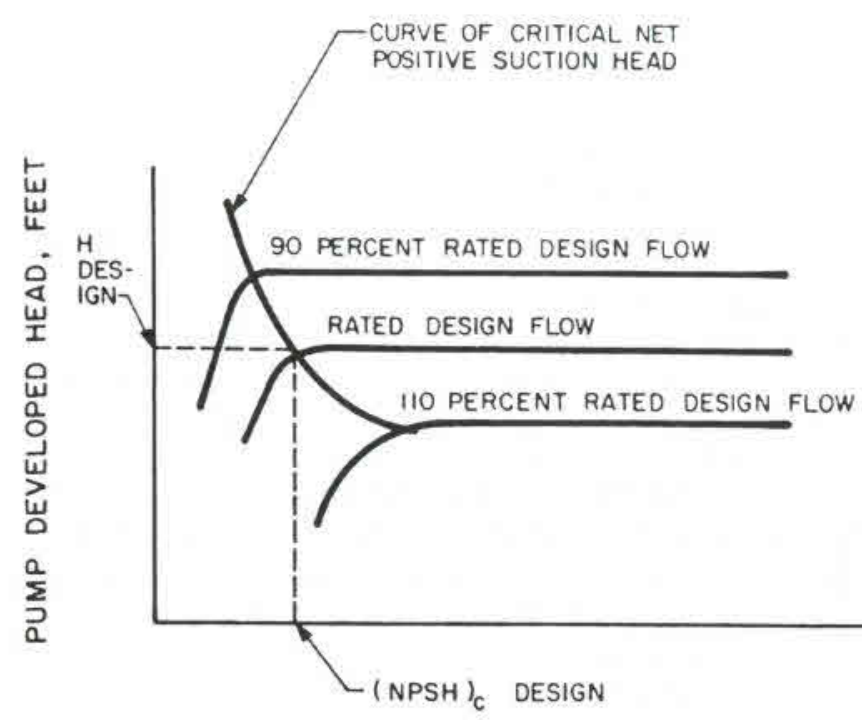

Fig. 6-21 Typical cavitation characteristics of a pump operated at rated design speed.

$$
\begin{aligned}
& \text { where- } \\
& (\mathrm{NPSH})_{\mathrm{a}}=\text { available net positive suction head, } \mathrm{ft} \\
& \mathrm{Pt}=\text { propellant-tank pressure, psia } \\
& \rho=\text { density of propellant, } \mathrm{lb} / \mathrm{ft}^{3} \\
& \mathrm{Z}=\text { height of propellant above the pump }
\end{aligned}
$$

Alternatively, total pressure at the pump inlet given by $\mathrm{P}_{\mathrm{t} 1}$, NPSH can be simply expressed as-

$$
\mathrm{NPSH}_{\mathrm{a}}=\left(\mathrm{P}_{\mathrm{t} 1}-\mathrm{p}_{\mathrm{v}}\right) 144 / \rho
$$

In design practice, the term "critical net positive suction head" $(\mathrm{NPSH})_{\mathrm{C}}$ defines the NPSH value that will result in a $2 \%$ head-generation loss at the rated design speed and flow rate of a given pump. (Some references use a $3 \%$ head falloff rather than $2 \%$.) Usually further reduction in inlet NPSH below the (NPSH)c point results in rapidly increasing cavitation. In turn, the developed head is further reduced, bringing on nonsteady flow. Figure 6-21 represents the cavitation characteristics of a typical pump operated at rated design speed. Although the head produced by the pump remains relatively constant down to the critical NPSH, there may be significant cavitation occurring in the pump that can produce other effects. This is particularly true of inducers, which are designed to operate with cavitation. However, the cavitation can produce physical damage to the hardware and increase oscillations of both flow and pressure in the pump. It is not uncommon to be able to visually see cavitation in inducers at NPSH values that are several times the 
critical, and the oscillations are typically noticeable in the range from one to two times the critical. These effects are dependent on the design, and it is not necessary, in general, to eliminate all cavitation to achieve a good design even for applications requiring long life. In fact, most rocket-engine pumps operate with cavitation to minimize weight. Below the critical NPSH, surging flow can occur or pump speed can run away due to the loss of head in the pump. Operation below this value must be carefully monitored.

To ensure a margin of safety for pump operation, the (NPSH)a supplied by the vehicle must be larger than the (NPSH) $)_{c}$ of the propellant pumps. The margin to be used will depend on the application. General guidelines are presented in the section on inducer design.

It is useful to compare the suction characteristics of various pump designs on the basis of a design parameter called suction specific speed $\mathrm{N}_{\mathrm{SS}}$, which is defined as follows:

$$
\mathrm{N}_{\mathrm{SS}}=\mathrm{N}(\mathrm{Q})^{0.5 /(\mathrm{NPSH})} \mathrm{C}^{0.75}
$$

where-

$$
\begin{aligned}
\mathrm{N}_{\mathrm{SS}} & =\text { pump suction specific speed } \\
\mathrm{N} & =\text { pump rated design speed, rpm } \\
\mathrm{Q} & =\text { pump rated design volume flow rate, } \\
& \text { gpm } \\
(\mathrm{NPSH})_{\mathrm{C}}= & \text { pump critical net positive suction } \\
& \text { head, } \mathrm{ft}
\end{aligned}
$$

Suction specific speed is related to the critical net positive suction head in the same manner the specific speed is related to overall pump-developed head. Design values of suction specific speeds for rocket-propellant pumps range from 10,000 without inducers to values over 100,000 for certain propellants with inducers.

Another coefficient describing pump suction characteristics, the Thoma parameter $r$, is the ratio of critical net positive suction head $(\mathrm{NPSH})_{\mathrm{C}}$ and rated pump-developed head $\Delta \mathrm{H}$ :

$$
\mathrm{r}=(\mathrm{NPSH})_{\mathrm{C}} / \Delta \mathrm{H}=\left(\mathrm{N}_{\mathrm{S}} / \mathrm{N}_{\mathrm{SS}}\right)^{1.333}
$$

This parameter is not commonly used today because it does not represent a common pump characteristic.

For a given vehicle (NPSH)a, the pump suction characteristics $\mathrm{N}_{\mathrm{SS}}$ determine the maximum permissible rpm at the design flowrate. A high pump $\mathrm{N}_{S S}$ capability or a high vehicle (NPSH)a permits a higher pump rpm, lower turbopump weight, and possibly higher turbine performance. It is desirable to operate a pump at the highest practical speed. Figure 6-22 represents the results of a parametric turbopump-system design study for a typical $\mathrm{LO}_{2} / \mathrm{RP}-1$ booster-stage rocket, such as the A-1 Stage engine. The study reflects the effects of N, (NPSH) , and $\mathrm{N}_{\mathrm{SS}}$ on the selection of turbopump configuration.

In addition to the pump (NPSH) ${ }_{C}$ values during steady-state operation, the engine starting transient pump $(\mathrm{NPSH})_{\mathrm{c}}$ must be determined and specified to permit satisfactory pump acceleration from zero to nominal design speed and flow rate in the desired time and manner. The starting (NPSH) $\mathrm{C}$ depends on the rate of acceleration and on the control system of the engine, as well as on vehicle acceleration, gravity effects, and propellant-suction-duct geometry. Therefore, sufficient tank pressure must be provided to accelerate the propellant and to overcome the hydraulic resistance in the suction duct, as well as to supply the necessary pump (NPSH) ${ }_{C}$ during all phases of system operation. Similarly, the NPSH during shutdown transients must be sufficient to permit a safe shutdown without a turbopump-overspeed condition.

Pump operational efficiency; losses. Several types of energy loss occuring during pump operation affect efficiency.

Hydraulic losses. These include friction losses in the passages and flow-turbulence losses. The friction losses are a function of the "wetted areas" in the passages and of the roughness of their surfaces. The turbulence losses are caused by disturbances in certain regions of the pump, such as at the inlet and outlet edge of the vanes of both impeller and diffuser and in the return guide-vanes. Flow in pump components is essentially always diffusing (lower relative velocity at the exit plane than at the inlet for a given blade row), which has associated losses. Tipclearance flows contribute losses in unshrouded regions. Cavitation in flow passages can increase these losses by causing local accelerations plus adding the losses associated with the cavitationcollapse mechanism.

Disk-friction losses. The energy required to rotate a disk, such as an impeller or inducer, in a fluid is known as disk-friction loss. The disk-friction losses are due to two actions: the actual friction of fluid on the disk, which is relatively minor, and a pumping action on the fluid in contact with the disk, whereby the fluid is circulated locally by centrifugal action. The energy loss due to disk friction is transformed into heat and can appreciably increase the temperature of the fluid.

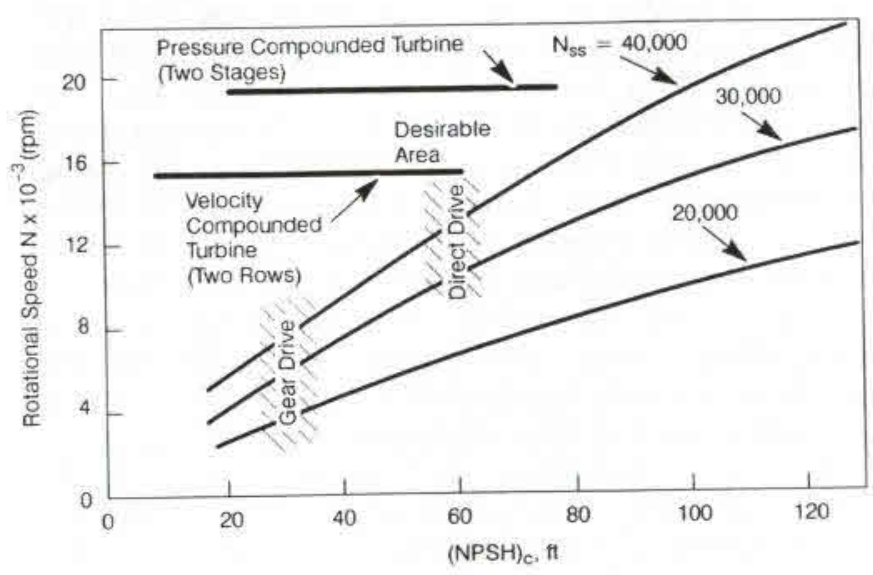

Fig. 6-22 Effects of N, (NPSH) $c$ and $\mathrm{N}_{\mathrm{Ss}}$ on turbopump selection for a typical $\mathrm{LO}_{2} / \mathrm{RP}-1$ booster-stage rocket engine system. 
Mechanical losses. These are losses in bearings and seals caused by mechanical friction.

Leakage losses. To prevent the pumped fluid from leaking back to the inlet after it has exited the impeller and is at outlet-pressure levels, closeclearance seals or wearing rings are provided. Increases in leakage require pumping power and introduce heat into the inlet fluid; both contribute to a loss in efficiency.

The overall efficiency of a pump $\left(\eta_{p}\right)$ can be expressed by the ratio of pump fluid-horsepower output, fhp, to brake-horsepower input by the pump drive, bhp:

$$
\eta_{p}=f h p / b h p
$$

The pump fluid horsepower-the actual usable output delivered by the pump-is the product of delivered propellant weight flow $\left[\mathrm{w}_{\mathrm{p}}(\mathrm{lb} / \mathrm{s})\right]$ times the actual head $\Delta \mathrm{H}(\mathrm{ft})$ developed by the pump, divided by a conversion factor:

$$
\text { fhp }=w_{p} \Delta H / 550
$$

The brake horsepower represents the mechanical horsepower delivered to the pump by the drive. It is consumed in the pump as fluid horsepower and as the various losses:

$$
b h p=f h p+(h p)_{h}+(h p)_{d f}+(h p)_{m}+(h p)_{l}
$$

$$
\begin{aligned}
\text { where- } & \text { brake horsepower } \\
\text { bhp } & =\text { fluid horsepower } \\
\text { fhp } & \text { horsepower required to overcome } \\
(\mathrm{hp})_{\mathrm{h}}= & \text { hydraulic losses } \\
(\mathrm{hp})_{\mathrm{df}}= & \text { horsepower required to overcome disk } \\
& \text { friction losses } \\
(\mathrm{hp})_{\mathrm{m}}= & \text { horsepower required to overcome } \\
& \text { mechanical losses } \\
(\mathrm{hp})_{1}= & \text { horsepower required to overcome } \\
& \text { leakage loss }
\end{aligned}
$$

The efficiency of a pump is related to the volume flow rate $Q$ and the pump specific speed $N_{S}$. There is a definite trend toward increased efficiency with higher pump capacities primarily because the resulting larger size of the pump permits operation with smaller relative clearances, reducing leakage losses, and surfaces that are hydrodynamically smoother, reducing the skin-friction losses. The lowspecific-speed pumps have less efficiency because they typically have disk-friction and leakage losses that are relatively higher. This can be seen from Fig. 6-23, which shows the efficiency as a function of both the specific speed and the volumetric flowrate. (All rocket-engine pumps do not fall exactly on these curves, but they show the correct trends.) The overall efficiency of rocket-engine propellant pumps of high developed head and rotating speed ranges from 60 to $85 \%$. This is up to $10 \%$ lower than that of highperformance industrial pumps. For a typical centrifugal pump, Fig, 6-24 shows the correlation between pump flowrate and the following three parameters: developed head, efficiency, and required brake horsepower.

Turbine overall performance and operating efficiency. The turbine generates the power needed to rotate the pump at the desired speed. The turbine derives its energy from the working fluid. The power generated equals the product of the flow times the available energy of the working fluid times the turbine efficiency. To achieve the desired power, the turbine designer will typically try to achieve the maximum efficiency within the constraints imposed, and the tradeoff between flow and available energy will depend on the particular cycle.

The available energy content of the turbine working fluid is defined as the enthalpy drop per pound of working fluid in the turbine:

$$
\Delta \mathrm{H}_{\mathrm{t}}=\mathrm{H}_{\mathrm{O}}-\mathrm{H}_{\mathrm{e}}
$$

where-

$\Delta H_{t}=$ available energy content of the working fluid, Btu/lb

$\mathrm{H}_{\mathrm{O}}=$ enthalpy per unit weight of the working fluid at turbine inlet, Btu/lb

$\mathrm{H}_{\mathrm{e}}=$ enthalpy per unit weight of the working fluid at exhaust pressure (assuming isentropic expansion), Btu/lb

Using Eq. (1-10) and (1-13), Eq. (6-12) can be rewritten for an ideal gas as follows:

$$
\Delta H_{t}=C_{p}\left(T_{o}-T_{c}\right)=C_{p} T_{o}\left[1-\left(\frac{p_{e}}{p_{o}}\right)^{\frac{\gamma-1}{\gamma}}\right]
$$

where-

$\mathrm{C}_{\mathrm{p}}=$ working-fluid specific heat at constant pressure, Btu/lb-F

$\mathrm{T}_{\mathrm{O}}=$ working-fluid total temperature at turbine inlet, ' $\mathrm{R}$

$\mathrm{T}_{\mathrm{e}}=$ working-fluid static temperature at turbine exhaust, "R

$\mathrm{p}_{\mathrm{O}}=$ working-fluid total pressure at turbine inlet, psia

$\mathrm{Pe}=$ working-fluid static pressure at turbine exhaust, psia

$\gamma=$ working-fluid specific heat ratio

The ratio of turbine-inlet and exhaust pressures $\mathrm{p}_{1} / \mathrm{p}_{2}$ can be expressed as the turbine pressure ratio $\mathrm{R}_{\mathrm{t}}$, which is a frequently used parameter in turbine design. Substituting this into Eq. (6-13) yields-

$$
\Delta \mathrm{H}_{\mathrm{t}}=\mathrm{C}_{\mathrm{p}} \mathrm{T}_{\mathrm{o}}\left[1-\left(\frac{1}{\mathrm{R}_{\mathrm{t}}}\right)^{\frac{\gamma-1}{\gamma}}\right]
$$

It can be seen that the available energy in the turbine working fluid is a function of gas properties, inlet temperature, and the turbine pressure ratio. 


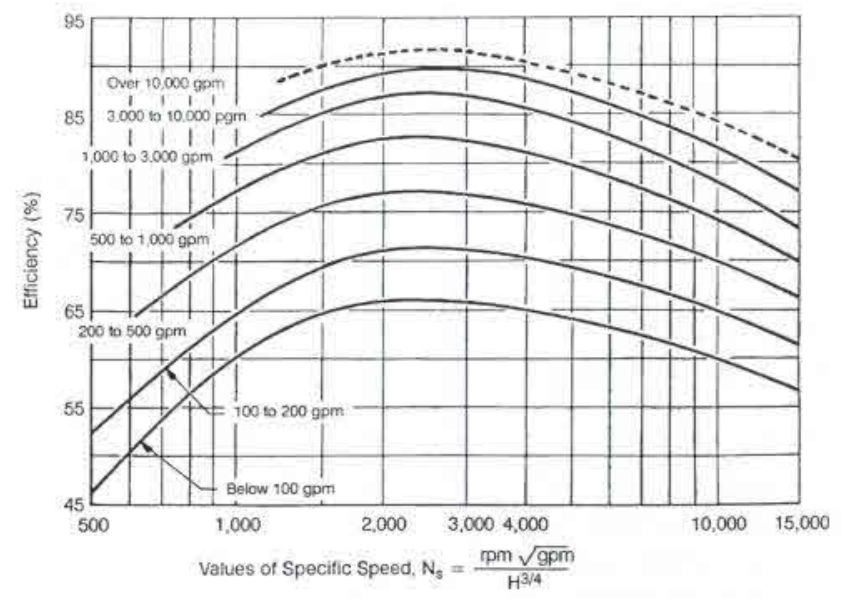

Fig. 6-23 Variation of pump efficiency with specific speed.

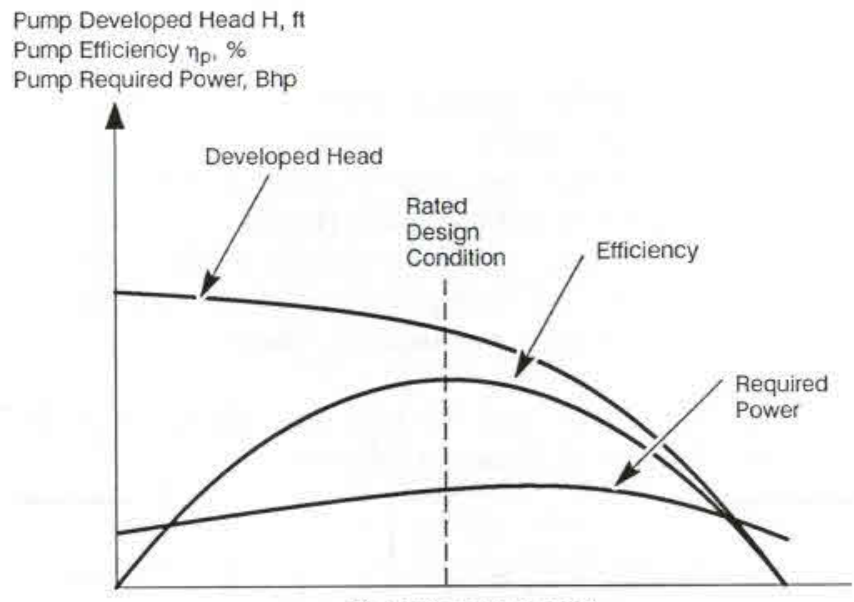

Pump Flowrate $Q$ (gpm)

Fig. 6-24 H-Q, efficiency, and required power characteristic curves of a typical centrifugal pump.

Most of the turbine working fluids for rocket-engine application are fuel-rich product gases generated by bipropellant combustion. Typical working-fluid properties are listed in Table 6-4. Precaution: these working fluids do not behave exactly as perfect gases; real fluid properties must be used to assess accurately a turbine's performance.

Figure 6-25 shows the relationship between turbine-inlet temperature and available working-fluid energy for a turbine pressure ratio of 20 for the two propellant combinations, $\mathrm{LO}_{2} / \mathrm{RP}-1$ and $\mathrm{LO}_{2} / \mathrm{LH}_{2}$. Turbine-inlet temperatures are limited by the hightemperature properties of the turbine materials. State-of-the art uncooled metallic designs have a practical design limit of about $1600^{\circ} \mathrm{F}$. Above certain levels, the gain from a higher turbine-inlet temperature will be offset by the turbine efficiency losses (see "blade losses" in following paragraphs) resulting from higher gas-jet speed (spouting velocity $\mathrm{Co}$ ), which is proportional to the turbine-inlet temperature.
Table 6-4 Properties of typical fuel-rich combustion product gases.

\begin{tabular}{|c|c|c|c|c|c|}
\hline Fluid & $\begin{array}{c}\text { Inlet } \\
\text { Temper- } \\
\text { ature } \\
\text { 'F }\end{array}$ & $\begin{array}{l}C_{p} \text {; } \\
\text { Btul } \\
\text { lb 'F }\end{array}$ & $\gamma$ & $\underset{\mathrm{ft} \mathrm{J}^{\prime} \mathbf{R}}{\mathbf{R}}$ & $\begin{array}{c}\text { Mixture } \\
\text { Ratio, } \\
\text { O/F }\end{array}$ \\
\hline \multirow[t]{13}{*}{ LOX/RP-1 } & 1100 & 0.635 & 1.097 & 43.3 & 0.308 \\
\hline & 1150 & 0.639 & 1.100 & 45.1 & 0.320 \\
\hline & 1200 & 0.643 & 1.106 & 47.1 & 0.337 \\
\hline & 1250 & 0.646 & 1.111 & 58.6 & 0.354 \\
\hline & 1300 & 0.648 & 1.115 & 50.4 & 0.372 \\
\hline & 1350 & 0.651 & 1.119 & 51.8 & 0.390 \\
\hline & 1400 & 0.653 & 1.124 & 53.6 & 0.408 \\
\hline & 1450 & 0.655 & 1.128 & 55.4 & 0.425 \\
\hline & 1500 & 0.657 & 1.132 & 58.0 & 0.443 \\
\hline & 1550 & 0.659 & 1.137 & 59.0 & 0.460 \\
\hline & 1600 & 0.660 & 1.140 & 60.7 & 0.478 \\
\hline & 1650 & 0.661 & 1.144 & 62.4 & 0.497 \\
\hline & 1700 & 0.662 & 1.148 & 64.0 & 0.516 \\
\hline \multirow[t]{6}{*}{$\mathrm{N}_{2} \mathrm{O}_{4} / \mathrm{CH}_{2}(\mathrm{UDMH})$} & 1400 & 0.380 & 1.420 & 87.5 & 0.110 \\
\hline & 1500 & 0.398 & 1.420 & 91.6 & 0.165 \\
\hline & 1600 & 0.416 & 1.420 & 95.7 & 0.220 \\
\hline & 1700 & 0.434 & 1.420 & 99.9 & 0.274 \\
\hline & 1800 & 0.452 & 1.420 & 104.0 & 0.328 \\
\hline & 1900 & 0.470 & 1.420 & 108.2 & 0.382 \\
\hline \multirow[t]{6}{*}{$\mathrm{LOX}^{\mathrm{L} \mathrm{H}_{2}}$} & 1000 & 2.05 & 1.374 & 434 & 0.785 \\
\hline & 1200 & 1.94 & 1.364 & 403 & 0.908 \\
\hline & 1400 & 1.86 & 1.354 & 378 & 1.025 \\
\hline & 1600 & 1.80 & 1.343 & 358 & 1.143 \\
\hline & 1800 & 1.73 & 1.333 & 336 & 1.273 \\
\hline & 2000 & 1.69 & 1.322 & 320 & 1.410 \\
\hline
\end{tabular}

Figure 6-26 shows the relationship between turbine pressure ratio and available energy of the working fluid for a turbine-inlet temperature of $1200^{\circ} \mathrm{F}$, again for the propellant combinations $\mathrm{LO}_{2} / \mathrm{RP}-1$ and $\mathrm{LO}_{2} / \mathrm{LH}_{2}$. Although a large amount of energy may be available in a working fluid, it may be difficult to convert it efficiently into turbine shaft horsepower because of the severe weight limitations on rocket-engine turbopumps. Thus the available pressure ratio of a turbine often cannot be fully used.

The overall efficiency of a turbine $\eta_{t}$ is defined as the ratio of turbine shaft horsepower thp to enthalpy drop rate, or the available energy-delivered rate of the working fluid:

$$
\eta_{\mathrm{t}}=\frac{550 \mathrm{thp}}{778 \dot{\mathrm{w}}_{\mathrm{t}} \Delta \mathrm{H}_{\mathrm{t}}}
$$

Again, for an ideal gas, this can be expressed using Eq. (6-14) to show the relationship to fluid properties, as follows:

$$
\eta_{\mathrm{t}}=0.707 \frac{\mathrm{thp}}{\dot{\mathrm{w}}_{\mathrm{t}} \mathrm{C}_{\mathrm{p}} \mathrm{T}_{\mathrm{O}}\left[1-\left(\frac{1}{\mathrm{R}_{\mathrm{t}}}\right)^{\frac{\gamma-1}{\gamma}}\right]}
$$

In turbine operation, efficiency can be affected by the following losses:

- Nozzle losses. Similar to those in thrust-chamber 


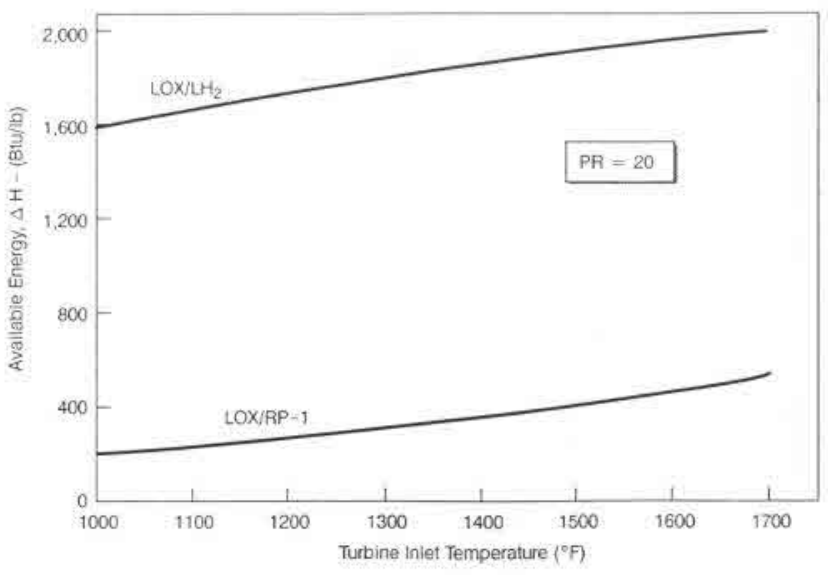

Fig. 6-25 Effect of turbine-inlet temperature on working-fluid available energy.

nozzles, these losses are due to the gas leaving the nozzle at a lower velocity than it would an ideal nozzle. The losses are due to flow turbulence, fluid friction, fluid turning, and loss of heat to and through the turbine nozzle-blocks.

- Blade losses. These are caused by residual velocity of the gas as it leaves the rotor blades, the obliquity of the nozzle (i.e., the nozzle angle in Fig. 6-10 cannot be made zero), flow turbulence, and fluid friction. Losses due to residual gas velocity can be reduced by optimizing the turbine blade-to-gas velocity ratio $\mathrm{U} / \mathrm{Co}$, where $\mathrm{U}$ is the pitch speed of the rotor blades and $\mathrm{Co}$ is the ideal spouting velocity of the gas based on available energy and isentropic expansion. These conditions can be analyzed quantitatively by means of turbine-gas-flow velocity diagrams (to be discussed in section 6.6). Flow turbulence can be reduced through improved blade shape and through full turbine nozzle admission.

- Leakage or clearance losses. The clearance required between rotor-blade tips and casing permits some gas to leak past the blades without doing work, thus causing energy losses. The gas leakage from stage to stage in a multistage pressure-compounded turbine due to required clearance between shaft and sealing diaphragm results in similar losses.

- Disk-friction losses. Fluid friction occurs at the interface of gas and rotor-disk surfaces. Also, centrifugal action of the rotor disk causes some of the gas to flow radially to the casing and to be dragged along the face of the casing by the rotor blades, causing friction.

- Mechanical losses. These result from the mechanical friction in bearings and rotating seals.

Efficiency. The design of turbines for rocket turbopumps tends toward the simpler and lighter impulse-types, with most of the expansion occurring in the stationary elements. Figure 6-27 shows the typical efficiency curves of various impulse-type turbines. The design problem becomes one of balancing efficiency (optimum velocity ratio $\mathrm{U} / \mathrm{Co}$ ), weight (number of stages or rows), and structural considerations. A higher performance can be achieved by employing a working fluid with high

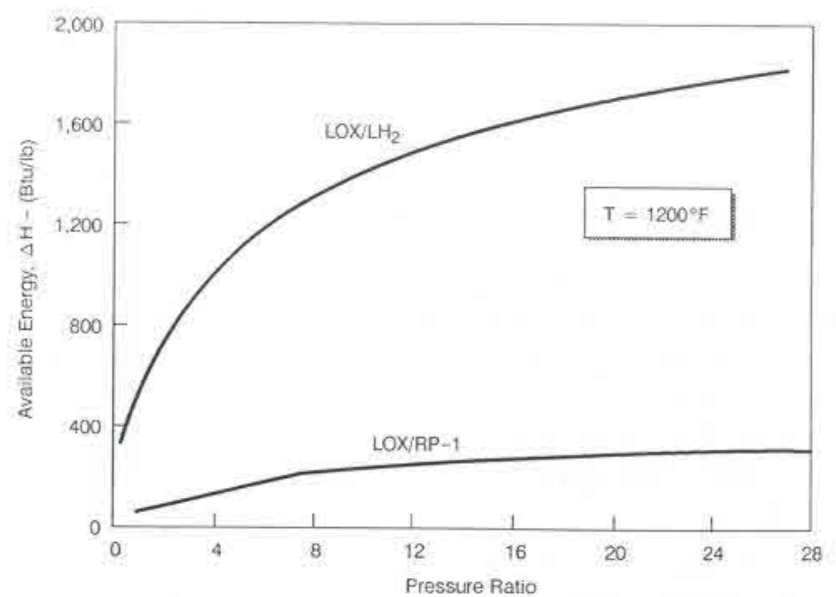

Fig. 6-26 Effect of turbine pressure ratio on working-fluid available energy.

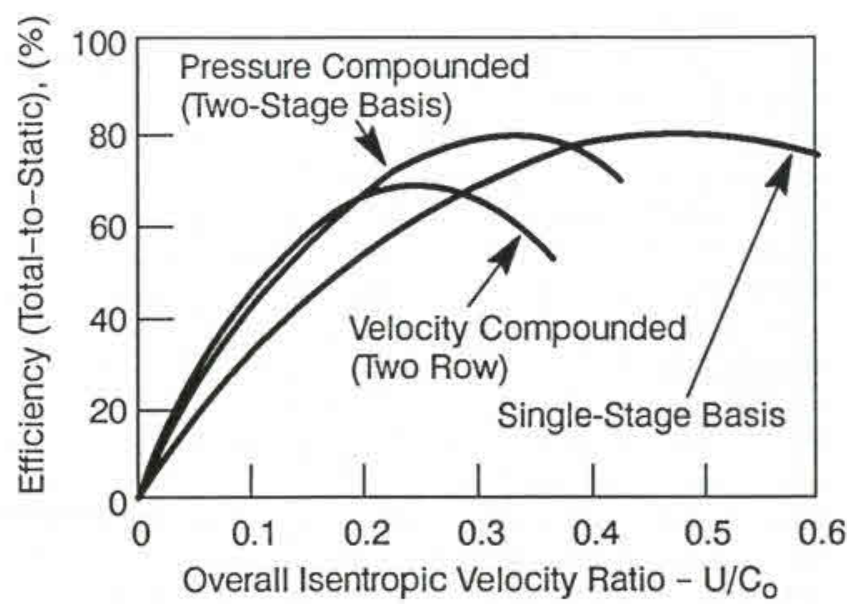

Fig. 6-27 Typical efficiency curves of impulsetype turbines.

available energy, and by matching its high gasspouting velocity Co with a high rotor-blade pitch speed $\mathrm{U}$. However, the blade speed is often limited by the required pump rpm, by the practical size of the rotor wheel, and the strength of materials. The optimum velocity ratio (or optimum value of blade speed for a given gas-spouting velocity) is reduced by the use of velocity or pressure-compounded arrangements (shown schematically in Fig. 6-9 and 611). However, these designs increase weight and complexity.

In general, a direct-drive configuration (Fig. 6-15) with an rpm lower than ideal produces a low value of $\mathrm{U} / \mathrm{Co}$, and a velocity-compounded turbine will be used because of its low overall weight and simplicity. Where a reduction gear is provided between pumps and turbine, a higher value of U/Co is possible and a more efficient pressure-compounded turbine can be used.

Since the turbine pressure ratio has only a small effect upon available energy content of the working fluid (Fig, 6-26), the power level of a turbine will usually be regulated by controlling the inlet pressure (po) and in turn the flow rate $\left(\mathrm{w}_{\mathrm{t}}\right)$ of the turbine. 
System calibration and off-design characteristics. Before the 1970s, rocket-engine systems operated at a single set of conditions, specifically, at rated thrust and mixture ratio. Each of the components in turn were designed for optimum functioning at that rated thrust level. However, most of the engine components, notably pumps and turbines, were also required to operate satisfactorily within a certain range different from the design point. Such a need may be caused by system and component calibration characteristics, system operational deviations, system start and shutdown transients, and special system requirements, such as throttling.

Owing to tolerance requirements on engine and component performance, and to correct for hydrodynamic variations within the components, it is sometimes necessary to calibrate a turbopump before its integration into an engine system. The performance of each pump will be determined experimentally. By modifying the pump geometry (impeller trimming) and varying the discharge hydraulic resistances, the desired operating characteristics required by the engine system can be achieved. For turbopumps with both pumps driven by a single turbine (as shown in Fig. 6-15), the calibration procedure begins with the experimental determination of the shaft speed at which one of the pumps (usually the oxidizer pump) develops the required head and flow. Simultaneously, the suction characteristics of this pump are determined at this speed. The other pump is then operated at the same speed and the discharge adjusted for the required flow. Based on the pressure readings, the diameter of the pump impeller will then be trimmed on a lathe until the desired head is produced at rated speed and flow. The pump suction characteristics at these conditions are also determined. Figure 6-28 shows the trimming effects of a typical pump. The trimming procedure requires that the pump impellers to be made sufficiently large initially, since addition of material is not feasible. The calibrated pumps are then combined with a turbine.

The turbine calibration requires either an adjustment of the flow to achieve the required power or, if the flow is limited, an adjustment of the fluid operating condition (e.g., temperature). This procedure considers the influence of turbine variations resulting from fabrication variations. The turbopump is then matched with the remaining engine system in complete-engine system calibrations. Adjustments in engine mixture ratio can be made by installing an orifice in the discharge of one of the pumps. Orifice installation considerations and type of propellant often make it desirable to ensure that the orificing is always installed for the same propellant; in LOX/RP systems, usually the fuel. For this reason, the pump for that system is trimmed for a slightly excess head. Adjustments in engine thrust level are made by varying the turbopump speed.

More recent engine designs (e.g., the SSME) require operation over a throttling range that forces the pumps to operate at off-design conditions. This requires an active control system that adjusts the respective pump parameters to achieve the desired operation. For the SSME, the oxidizer and fuel pumps are on separate shafts so that a separate adjustment of their respective speeds is achievable as well as an adjustment of flowrate.

One of the most significant pump off-design characteristics, the pump stall point, usually occurs in the low-flow region. The pump operation tends to be erratic at this point, resulting in the abrupt loss of developed head and the danger of overspeeding. Stall is the result of a boundary-layer separation at some point in the pump system. The first blade row to stall could be either a rotor or stator, depending on the particular design. The magnitude of the head falloff due to stall will also vary from pump to pump; but in general, axial-flow pumps tend to have a much more severe stall characteristic than centrifugal pumps, as illustrated in Fig. 6-29. Because of this, applications requiring a wide operating range typically use centrifugal designs rather than axial. It is the responsibility of the pump designer, of course, to establish the required operating range for a pump and to produce a design that will be satisfactory over this range.

During the start and shutdown transients of an engine system, propellant flow and pressure characteristics will be determined by the engine's systemdesign characteristics. Figure 6-30 shows the propellant flows and chamber-pressure buildup history for a typical gas-generator engine start transient (note temporary oxidizer-flow drop as a result of LOX-dome priming). A rocket turbopump must attain full power in substantially less time than conventional turbomachinery. Frequently, full-power operation must be attained in less than $1 \mathrm{~s}$-dictated by the need for propellant-consumption economy in flight and for avoiding the possibility of flow instability in the thrust chamber and other compo-

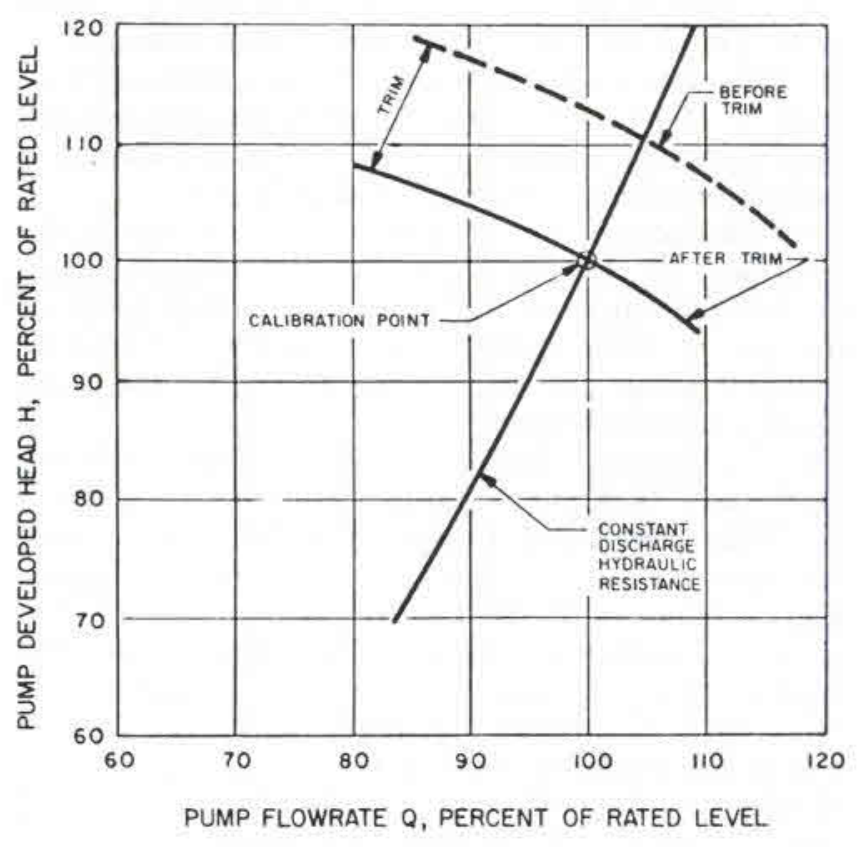

Fig. 6-28 Trimming effects of a typical pump. 


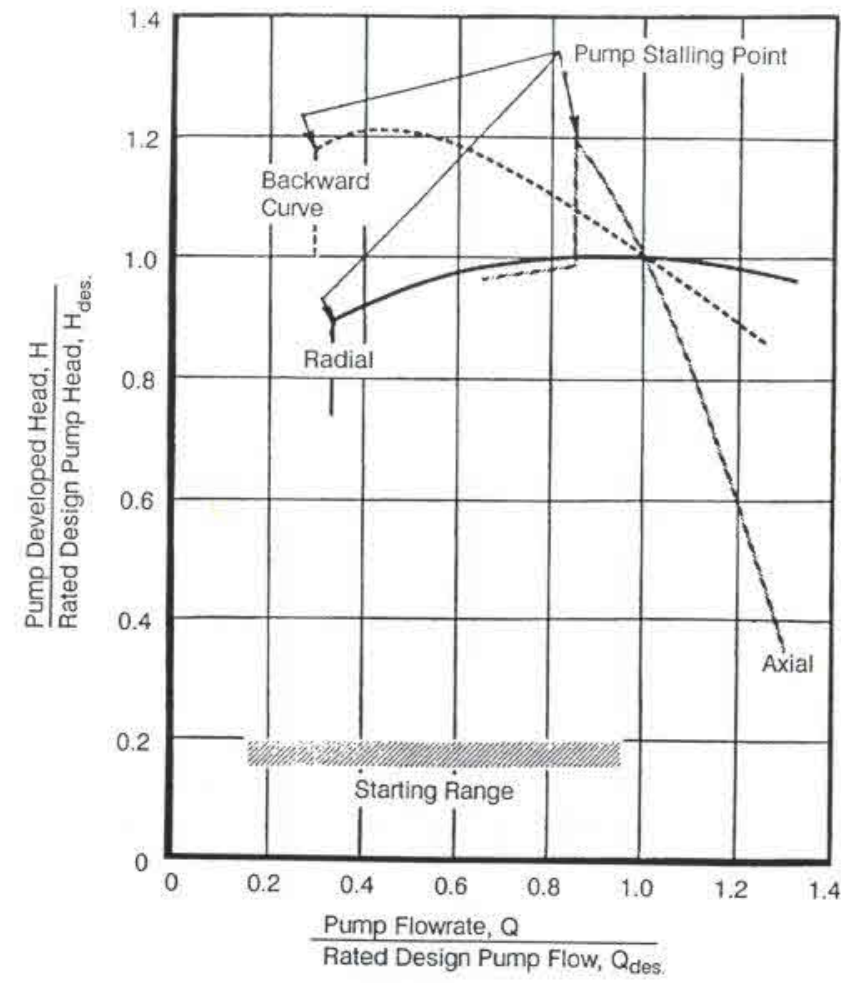

Fig. 6-29 Typical off-design characteristics of various types of pump.

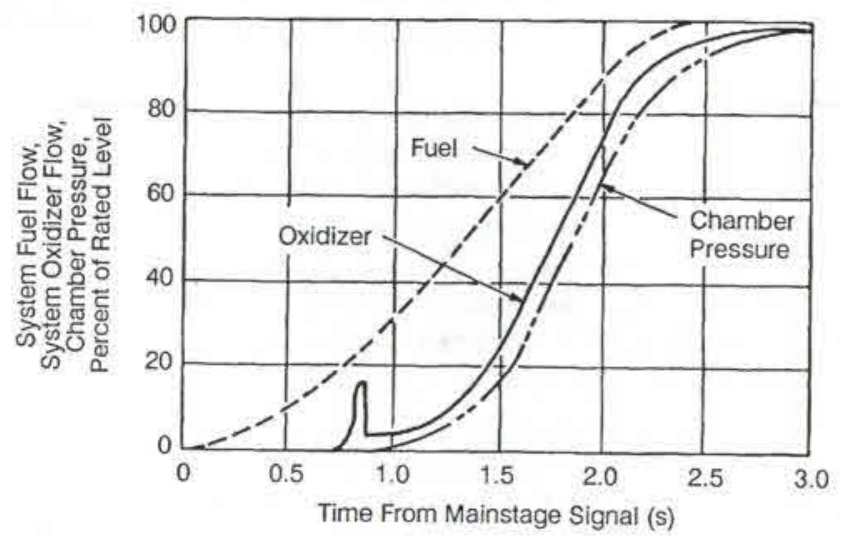

Fig. 6-30 Propellant-flow and chamberpressure transient characteristics during engine-system start.

nents. This, together with other transient requirements, such as throttling, must be satisfied by the offdesign characteristics of a turbopump. Throttling substantially influences selection of pump type. Basically, the problem involves coupling the pump characteristics with those of the rest of the engine system under off-design operating conditions.

Relative-velocity concepts. Before discussing the details of pump and turbine design, the concept of relative-velocity vectors must be understood because of the rotating blade rows. The rotating components have a velocity $U$ in the circumferential direction that is independent of flow. The velocity of the fluid relative to the rotor (W)

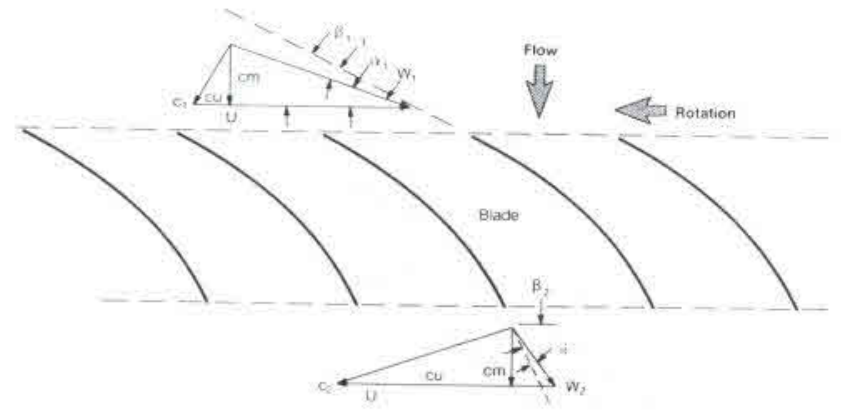

Fig. 6-31 Velocity diagrams for a pump.

plus the rotational velocity (U) equals the fluid velocity in the absolute reference plane. For example, Fig. 6-31 shows the absolute velocity at the inlet of a pump blade row as c with components $\mathrm{cm}$ in the through-flow direction and $\mathrm{cu}$ in the tangential direction (cu could be zero or even negative). The velocity $W_{1}$ is the inlet velocity relative to the blade that establishes the blade loading and performance. Similarly at the discharge, $\mathrm{W}_{2}$ is the relative vector experienced by the pump blade, but $c_{2}$ is the resulting absolute velocity. Note that, in the absolute plane, the velocity was increased $\left(c_{2}>c_{1}\right)$, i.e., the pump added energy to the flow. In the relative plane, however, the velocity is diffused (decreases, $\mathrm{W}_{2}<\mathrm{W}_{1}$ ). The boundary layers on the pump blade are based on the relative vectors, not the absolute vectors. These vector relationships will be used in further discussions of pump design.

\subsection{INDUCER DESIGN}

The rocket-engine industry has been the primary contributor to the development of inducer technology (Ref. 6-2). The inducer's axial-flow rotors can operate with a low inlet pressure without significant decrease in the discharge head. The inducer typically operates with some amount of cavitation, which may occur in the tip vortex flow or along the blade element itself as the inlet pressure is decreased. However, the technology has progressed to the point that long life can be achieved even with cavitation occurring. Inducers used in the initial rocket engines produced very low headrise with head coefficients that were typically less than 0.1 ; the inducer was operated in series, and generally was close-coupled to the main impeller. As the technology improved, inducer designs became more complex, with increased head coefficients (up to 0.25 with a single row of blades and in some cases over 0.5 with a double row of blades, as shown in Fig. 6-8). These larger head coefficients made it possible to use the inducer as a boost pump where the inducer is the only rotor on the shaft (e.g., LPOTP-see Fig. 6-7, and LPFTP pumps on the SSME). In some commercial applications, inducer pumps have been used where the only rotor is an inducer.

The need for an inducer is based on the required minimum NPSH, or alternatively, the maximum suction specific speed. Generally, an inducer will be required if the suction specific speed exceeds 

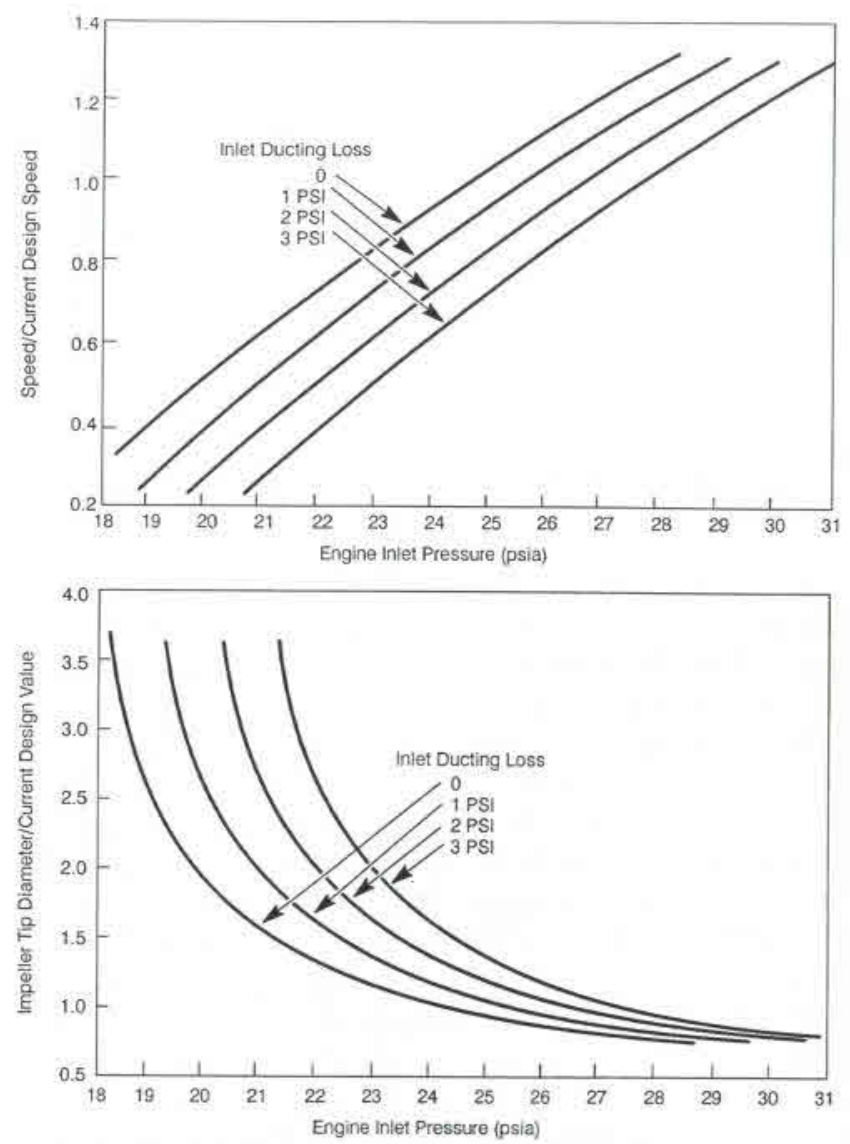

Fig. 6-32 Pump speed and diameter as a function of supplied inlet pressure.

10,000. For rocket-engine pumps that require suction specific speeds from 20,000 to 50,000 or higher, inducers are almost invariably used; and in fact, the suction performance will dictate the allowable speed of many turbopumps. This will in turn determine the size of the pump, number of stages, and general configuration to a large degree. Figure 6-32 shows the impact on speed and diameter of a given design as inlet pressure is reduced assuming constant head coefficient and number of stages for the pump.

Experience has shown that the suction of a pump strongly depends on its inlet flow coefficient. Figure 6-33 shows this effect for an inducer operating in a fluid with low vapor pressure (e.g., water). In cryogenics, the capability is even further improved because of the localized decrease in temperature and vapor pressure in the vicinity of the cavitation on the blade surface (an effect known as "thermodynamic suppression head"). This can be seen in Fig. 6-34, which shows actual data acquired from numerous pumps used in fluids with high vapor pressure, such as liquid hydrogen and oxygen. Some of the hydrogen pumps fall on the (NPSH $/ \mathrm{cm}^{2} / 2 \mathrm{~g}$ ) curve of 1.0 , reaching corrected suction-specificspeed values of up to 140,000 . Some of the LOX pumps fall on the curve of 2.0 .

Capabilities as expressed in Fig. 6-33 are based on some defined percentage of head loss in the pump. Common values used in industry are either 2 or $3 \%$ head falloff from the "noncavitating" head. If

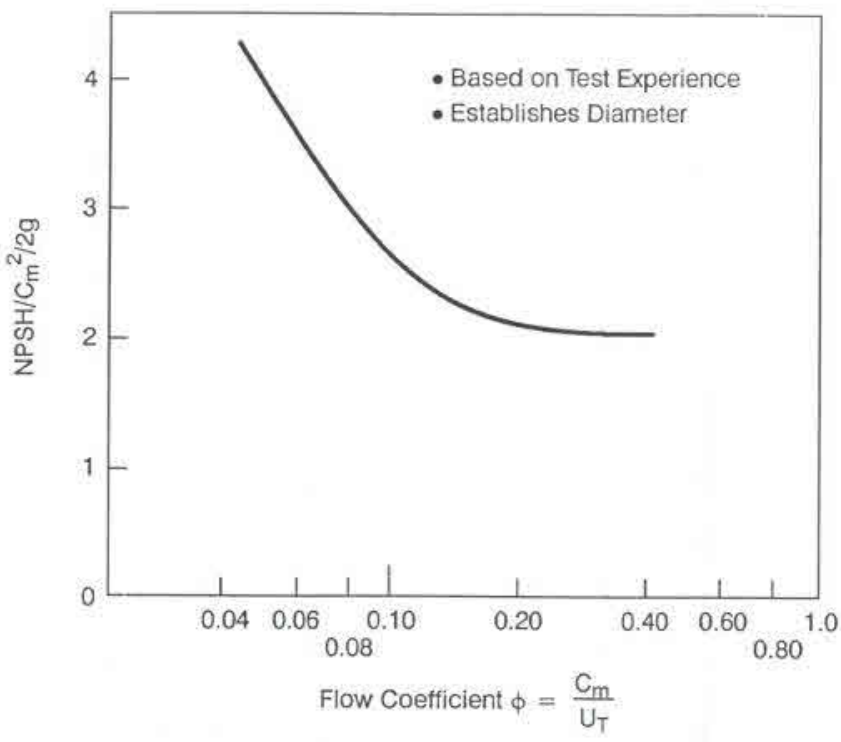

Fig. 6-33 Suction-specific-speed capability with no thermodynamic suppression head benefit.

the inducer is producing a small percentage of the overall head, as in a centrifugal or multi-stage axial pump, the total head lost by the inducer can be 15 to $30 \%$ when the pump head drops by $2 \%$. For boost pumps consisting of only the inducer, the head loss in the pump is equivalent to that for the inducer itself. The NPSH at which the defined head falloff occurs is called, as mentioned earlier, the critical $\mathrm{NPSH}\left(\mathrm{NPSH}_{\mathrm{C}}\right)$.

To design an inducer, the margin relative to the critical NPSH must be established based on the required use, life, and conservatism desired in the pump. For example, a pump designed for a nonmanrated expendable launch vehicle that only experiences the minimum NPSH at end of flight might be desired to operate at $\mathrm{NPSH}_{\mathrm{C}}$ at the end of the flight. For a reusable vehicle requiring long life and consistent operation at a given NPSH, however, a margin would be required, where the margin $M$ is expressed as a percentage using the relationship:

$\mathrm{M}=100\left(\mathrm{~min}\right.$. operating $\left.\mathrm{NPSH}-\mathrm{NPSH}_{\mathrm{C}}\right) / \mathrm{NPSH}_{\mathrm{C}}$

Typical values used in design would be $M=100 \%$ for long life at a given NPSH $=20 \%$ for short life at the minimum condition but ability to handle reasonable part-to-part and flight-to-flight variations.

To initiate an inducer design after defining the required margin, an experienced-based capability curve, as in Fig. 6-33 or 6-34, must be used. As was indicated above, fluids with a substantial vapor pressure (like liquid oxygen and liquid hydrogen) offer additional benefits. It has been shown that hydrogen inducers can operate at a critical NPSH equal to one inlet-velocity head, and liquid-oxygen inducers can operate with two inlet-velocity heads. That is, $\mathrm{NPSH}_{\mathrm{C}}=\mathrm{cm}^{2} / 2 \mathrm{~g}$ liquid hydrogen $=2 \mathrm{~cm}^{2} / 2 \mathrm{~g}$ liquid oxygen. However, this is only true when the inletvelocity head is small compared to the vapor head. 


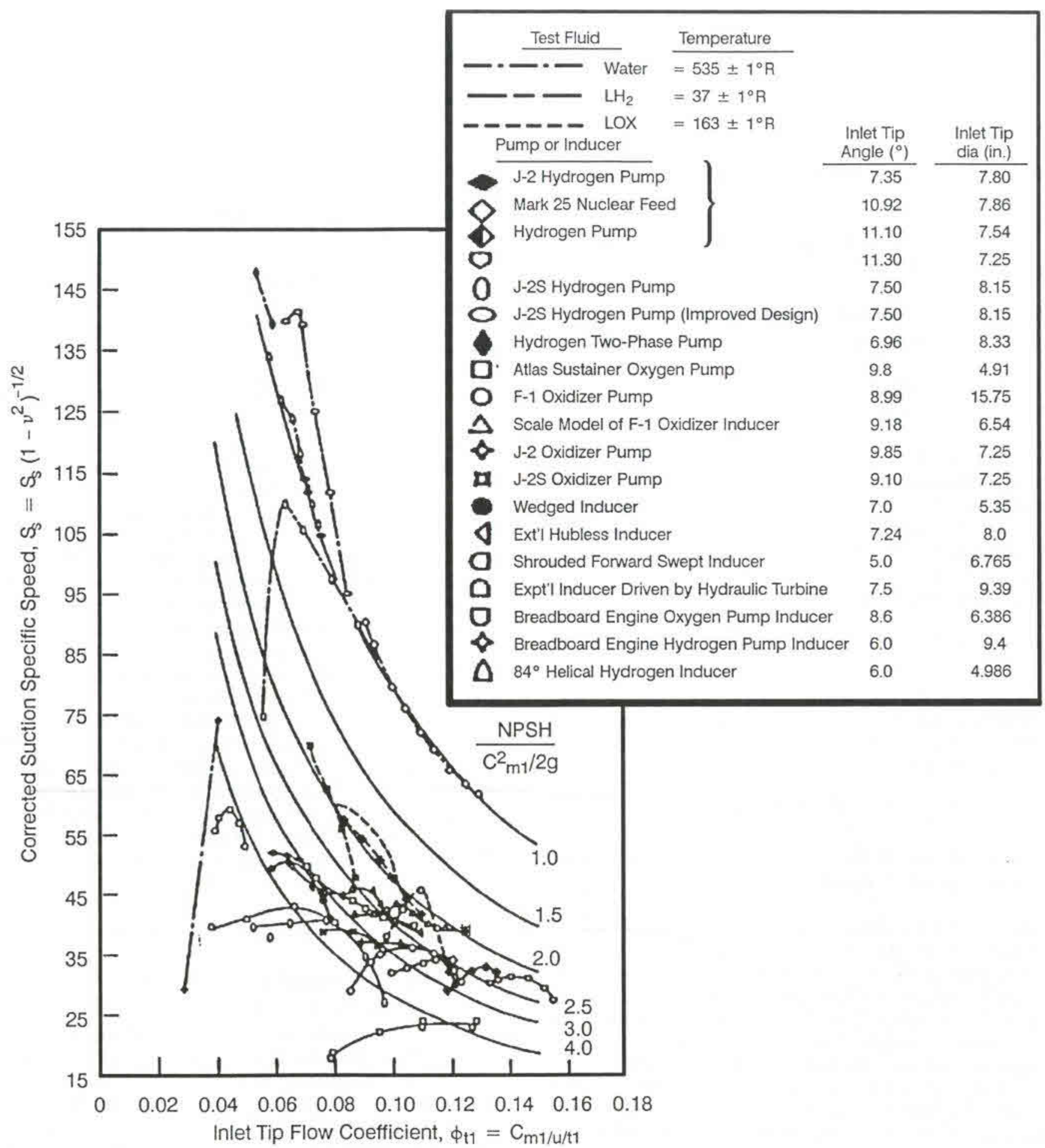

Fig. 6-34 Suction specific speed achieved in cryogenics.

As a general limit, the thermodynamic suppression head TSH of a fluid cannot exceed $50 \%$ of the vapor head.

To illustrate a typical design process, assume that an engine is being designed to deliver $25,000 \mathrm{gpm}$ of liquid oxygen with a minimum required total inlet pressure of 25 psia and a vapor pressure of 15 psia. Assume the design only operates at minimum conditions for a short time, nominally operating at much higher inlet pressure.
Let $M=20 \%$

NPSH minimum $=(25-15) \quad 144 / 70 \sim 20.6 \mathrm{ft}$

$\mathrm{NPSH}_{\mathrm{C}}=20.6 / 1.2=17.1 \mathrm{ft}$

Assume the inlet hub-diameter of the inducer is $30 \%$ of the tip diameter. For some high-speed inducers in a dense fluid like liquid oxygen, a larger value of $40 \%$ may be required for structural reasons. Calculate $\mathrm{cm}$ :

$$
\begin{aligned}
\mathrm{cm}= & \left(2 \mathrm{~g} \mathrm{NPSH} \mathrm{N}_{\mathrm{C}} / \mathrm{A}\right)^{0.5} \quad(\mathrm{~A}=1,2,3 \text { dependent on } \\
& \text { fluid; for } \left.\mathrm{LO}_{2} \text { use } 2\right) \\
\mathrm{cm}= & (2 \mathrm{~g}[17.1] / 2) 0.5=23.5 \mathrm{fps}
\end{aligned}
$$


Verify that inlet velocity head is less than 0.5 times vapor head to get full TSH benefit:

$$
\begin{aligned}
& \mathrm{cm}^{2} / 2 \mathrm{~g}=8.55 \mathrm{ft} \\
& 50 \% \text { of vapor head }=0.5(15)(144) / 70=15.4 \mathrm{ft}
\end{aligned}
$$

Calculate the tip diameter of the inducer inlet:

$$
\begin{aligned}
\mathrm{cm} & =\mathrm{Q} / \mathrm{A} \\
\mathrm{A} & =(\pi / 4) \mathrm{Dt}^{2}\left(1-\left[\mathrm{D}_{\mathrm{h}} / \mathrm{D}_{\mathrm{t}}\right]^{2}\right) / 144 \mathrm{ft}^{2} \\
& =4.96\left(10^{-3}\right) \mathrm{Dt}^{2}
\end{aligned}
$$

where $D_{t}$ is in units of inches and $Q$ must be in units of cfs. Substituting from the above values yields:

$$
\begin{aligned}
D_{t} & =21.86 \text { in. } \\
D_{h} & =6.56 \text { in }
\end{aligned}
$$

At this point, a flow coefficient must be assumed. For optimum suction performance, values in the range of 0.06 to 0.10 are used; the lowest values are more sensitive to fabrication tolerances and more limited in flow range. For long-life values higher than 0.15 are used. Inducers with values up to 0.3 have been tested. Assume:

$$
\begin{aligned}
\phi & =0.1 \\
U_{\mathrm{t}} & =\mathrm{cm} / \phi=235 \mathrm{ft} / \mathrm{s}
\end{aligned}
$$

This is a reasonable value for structural limits. The pump speed is then $\mathrm{N}=2464 \mathrm{rpm}$. Check suction specific speed:

$$
\mathrm{N}_{\mathrm{SS}}=\mathrm{N}(\mathrm{Q})^{0.5} /\left(\mathrm{NPSH}_{\mathrm{C}}\right)^{75}=46,300
$$

Based on Fig. 6-34, this is an achievable suction specific speed in liquid oxygen.

The head generated by the inducer usually will be selected to enable the following impeller to operate with essentially no cavitation. For example, the impeller might be designed for a maximum suction specific speed of 5000 to provide some margin in the impeller design. Thus, the head produced by the inducer must be sufficient to increase the NPSH from its inlet value to the value needed for the impeller. Using the numbers from the above example and letting $\mathrm{NPSH}_{\mathrm{I}}$ equal the NPSH at the impeller inlet yields-

$$
\begin{aligned}
\mathrm{N}_{\mathrm{SS}} & =5000=2464(25,000) 0.5 / \mathrm{NPSH}_{\mathrm{I}} 0.75 \mathrm{NPSH}_{\mathrm{I}} \\
& =332.8 \mathrm{ft}
\end{aligned}
$$

Therefore, the required inducer head rise will be $\Delta H$ $=332.8-20.6=312.2 \mathrm{ft}$.

With the tip speed of the inducer previously calculated, the inducer head coefficient will be $\psi=$ $312.2 /\left([235]^{2} / 32.2\right)=0.182$. This is an achievable head coefficient with a single row of blades. However, to achieve this it will be necessary to increase the diameter of the hub at the discharge.

The hub diameter of inducers for current rocketengine pumps will usually be larger at the discharge than at the inlet. This reduces the amount of diffusion being done along the hub streamline-a critical factor in preventing discharge recirculation and thereby providing a relatively uniform head and velocity into the impeller eye or into a downstream stator. The design objective is to achieve the head in the inducer, provide a relatively uniform discharge condition, avoid any recirculation at the inducer discharge, and avoid any cavitation on the leading edge of the downstream blades. The exact diameter selected will be based on analyses of the flow in both the inducer and impeller (or stator) using either a quasi-3D or full-3D approach.

Approximate selections can be based on velocity-vector diagrams at the inlet and exit of each blade row using simple radial-equilibrium equations at the discharge to account for variations from tip to hub. The resulting profile would appear as shown for the inducer in Fig. 6-7.

The number of blades for the inducer is kept small at the leading edge to minimize blockage and thus improve suction performance. Previous designs have used 3 to 7 inlet blades, but 3 to 4 is preferable for suction performance. Some inducers with three blades experience larger radial loads under partial cavitating conditions. In view of that, four blades are more frequently used in current designs. Larger flow coefficients $(\varphi>0.2)$ allow a larger number of blades. To increase head rise, partial-length blades are sometimes introduced downstream of the bladeoverlap region at the point where all cavitation is collapsed. Thus, the inducer exit would have twice the number of blades as the inlet. This is recommended if head coefficients must exceed 0.25 .

The inlet-velocity angle at the tip is established by the flow coefficient, assuming no inlet prewhirl. That is, letting $a_{i}$ equal inlet flow angle from tangential at the tip:

$$
\alpha_{\mathrm{i}}=\tan ^{-1} \mathrm{~cm} / \mathrm{U}_{\mathrm{t}}=\tan ^{-1} \phi
$$

With $\phi$ being generally less than 0.15 for rocketengine inducers, $\alpha_{i}$ is generally less than $8.5 \mathrm{deg}$. Inducers are designed to operate with an incidence angle $\mathrm{i}$ that varies from approximately $3 \mathrm{deg}$ up to a maximum of approximately one-half of the inlet blade angle. The effect of incidence angle on the design is discussed in detail in Ref. 6-2 through 6-5. The exact incidence chosen at the design point will depend on the required flow range of the part and the life characteristics. The inlet blade angle $\beta_{1 \mathrm{t}}$ equals the inlet flow angle plus the incidence angle, as shown in Fig. 6-31. The length of the blade is much longer in the tangential than in the axial direction because of the small blade angle. At the inlet, the blade angle varies from tip to hub according to the relationship-

$$
r \tan \beta=\text { constant }
$$

The discharge blade angle is set to achieve the desired head. This head can be expressed as-

$$
\Delta \mathrm{H}=\eta \Delta(\mathrm{Ucu}) / \mathrm{g}
$$


where $\eta$ is the inducer efficiency and the velocities $\mathrm{cu}$ are as illustrated in Fig. 6-31. The discharge axial velocity is calculated to satisfy continuity and radial equilibrium. The resulting velocities enable calculation of the discharge vector diagram. This establishes fluid velocities, but because of the blade loading the flow typically exits the inducer at an angle less than the blade angle. This difference (deviation angle) must be accounted for based on experience or an accurate Navier-Stokes solution of the inducer flow-field.

The distribution of blade angle between the inlet and discharge is designed to provide for both suction performance and efficiency. To improve suction performance, the inlet region to the point of overlap of the blades is typically designed with less camber than would be obtained, for example, from a circular-arc-blade profile. Final profiles are usually established based on quasi-3D or 3D analyses of the flow field.

The structural integrity of the blades must be evaluated over the full range of operation, including any transients. The blade loading due to partially cavitating conditions can be very different from the loads noncavitating. Typically, as inlet pressure decreases, more of the blade loading shifts to the back of the blading, owing to cavitation on the suction surface, which provides a minimum pressure limit.

\subsection{DESIGN OF CENTRIFUGAL PUMPS}

Because of its specific needs, the rocket industry has developed its own pump design approaches, which may differ from those for conventional applications. In addition, designers may employ their individual methods of analysis and calculation. However, the broad underlying principles are quite similar. The range of speeds, proportions, design coefficients, and other mechanical details for rocket-engine pumps has been well established by earlier designs as well as through experiments (Ref. 6-6).

\section{General Design Procedures}

As a rule, rated pump head capacity (H-Q) requirements and expected available NPSH at the pump inlets will be established by engine system design criteria. The first step then is to choose a suitable suction specific speed Nss and the type of inducer that will yield the highest pump speed $\mathrm{N}$ at design conditions [Eq. (6-7)]. The pump specific speed $\left(N_{S}\right)$ or type of impeller can now be established from the chosen pump speed and required head-capacity characteristics. Owing to its relatively light weight and simplicity of construction, a single-stage centrifugal pump may be given first consideration.

With suction specific speed and specific speed of the proposed pump design established, the designer can now look for a suitable "design model" among comparable existing pumps that approximate the desired performance (i.e., a pump with the same specific speed and suction specific speed). If a suitable model is available, the new pump design can be scaled from the previous design. Letting "f" equal the ratio of the scaled dimension, that is-

$$
\mathrm{f}=\mathrm{D}_{2} / \mathrm{D}_{1}
$$

makes the following correlations valid for pumps with like specific speed, based on the pump affinity laws [Eq. (6-3a) and (6-3b)]:

$$
\begin{aligned}
\mathrm{Q}_{2} & =\mathrm{Q}_{1} \mathrm{f}^{3}\left(\mathrm{~N}_{2} / \mathrm{N}_{1}\right) \\
\mathrm{DH}_{2} & =\mathrm{DH}_{1} \mathrm{f}^{2}\left(\mathrm{~N}_{2} / \mathrm{N}_{1}\right)^{2}
\end{aligned}
$$

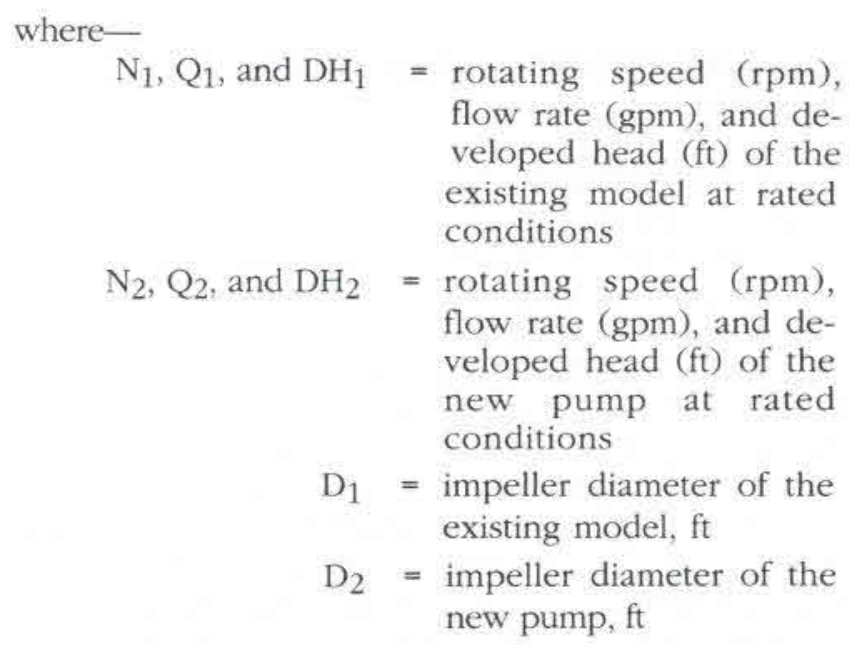

To achieve the same performance, all dimensions in the pump must be scaled by the scaling factor, including parameters such as surface finish and seal clearances. When scaling up, this is easily done, and usually the larger pump will achieve even better performance because of lower relative surface finish and clearance values. When scaling down, however, this works against the performance.

If a suitable model is not available for the design of a new pump, the designer can use "design factors" established experimentally by other successful designs. These may permit establishing relations between rated pump-developed head and flow rate and such parameters as velocity ratios. However, best results are obtained through experimental testing of the proposed design itself. The test results can then be used for design revisions and refinements. The discussion below uses the following basic symbols:
$c=$ flow velocity, absolute (relative to ducts and casing)
$\mathrm{v}=$ flow velocity, relative to inducer or impeller
$\mathrm{u}=$ velocity of points on inducer or im- peller

$\begin{aligned} \text { Subscript: } & \\ 0 & =\text { inducer inlet } \\ 1 & =\text { inducer outlet and impeller inlet } \\ 2 & =\text { impeller outlet } \\ 3 & =\text { pump casing exit } \\ \text { (prime) } & =\text { actual or design }\end{aligned}$




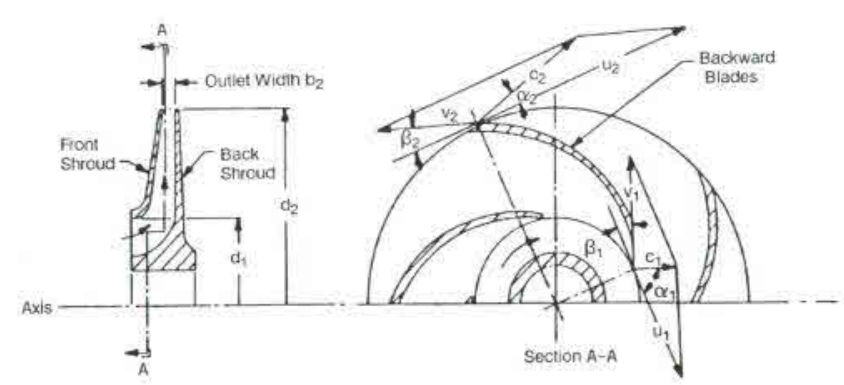

Fig. 6-35 Typical shrouded centrifugal impeller with backward-curved blades.

\section{Operating Principles of the Centrifugal Impeller}

In its simplest form, the impeller of a centrifugal pump can be regarded as a wheel with blades, rotating in an enclosure, with the fluid being admitted axially and ejected at the periphery, as shown schematically in Fig. 6-35. The tangential velocity component of the fluid increases as it moves out radially between the blades. The centrifugal force acting on the fluid therefore increases as the fluid moves out radially. For optimum performance, most impellers in high-speed centrifugal rocket-engine pumps have shrouded, backward-curved blades. The impeller width is tapered toward the periphery to keep the cross-sectional area of the radial flow path nearly constant, as shown in Fig. 6-35.

Velocity diagrams may be constructed to analyze the fluid flow vector correlations at various points of an impeller. Assume the following ideal conditions: no losses, such as fluid-friction losses, impeller passages completely filled with actively flowing fluid at all times, flow axisymmetrical (velocities at similar points on the flow lines are uniform), and fluid leaves the impeller passages tangential to the vane surfaces (complete guidance of the fluid at the outlet).

The ideal inlet- and outlet-flow velocity diagrams of the impeller described in Fig. 6-35 are shown in Fig. 6-36. With corresponding fluid velocities $\mathrm{u}, \mathrm{v}$, and $c$ (as identified above), $\alpha$ is the angle between $c$ and $u$, and $\beta$ is the angle enclosed by a tangent to the impeller vane and a line in the direction of vane motion. The latter is equal to the angle between $\mathrm{v}$ and $\mathrm{u}$ (extended). Based on these velocity diagrams, the following correlations have been established:

$$
\begin{aligned}
& \Delta \mathrm{H}_{\mathrm{ip}}=\left[\mathrm{u}_{2}{ }^{2}-\mathrm{u}_{1}^{2}+\mathrm{v}_{1}^{2}-\mathrm{v}_{2}{ }^{2} \mathrm{]} /(2 \mathrm{~g}) \quad \text { Eq. }(6-23)\right. \\
& \Delta \mathrm{H}_{\mathrm{i}}=\left[\mathrm{u}_{2}^{2}-\mathrm{u}_{1}^{2}+\mathrm{v}_{1}^{2}-\mathrm{v}_{2}^{2}+\mathrm{c}_{2}^{2}-\mathrm{c}_{1}{ }^{2}\right] /(2 \mathrm{~g}) \\
& =\left(\mathrm{u}_{2} \mathrm{Cu}_{2}-\mathrm{u}_{1} \mathrm{Cu}_{1}\right) / \mathrm{g} \\
& \text { Eq. (6-24) } \\
& Q_{\text {imp }}=448.8 \mathrm{~cm}_{1} \mathrm{~A}_{1}=448.8 \mathrm{~cm}_{2} \mathrm{~A}_{2} \text { Eq. (6-25) } \\
& \mathrm{cu}_{2}=\mathrm{u}_{2}-\mathrm{cm}_{2} /\left(\tan \mathrm{b} 2^{\prime}\right)
\end{aligned}
$$

where-

$\Delta \mathrm{H}_{\mathrm{ip}}=$ ideal static-pressure-head rise of the fluid flowing through the impeller due

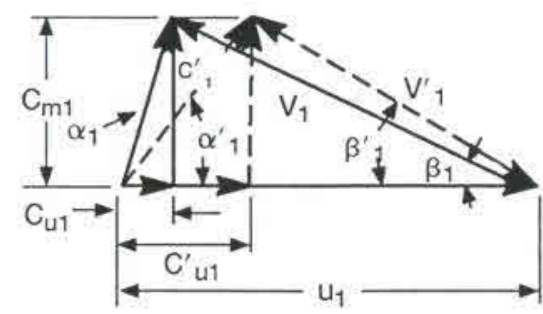

Inlet Velocity Diagram

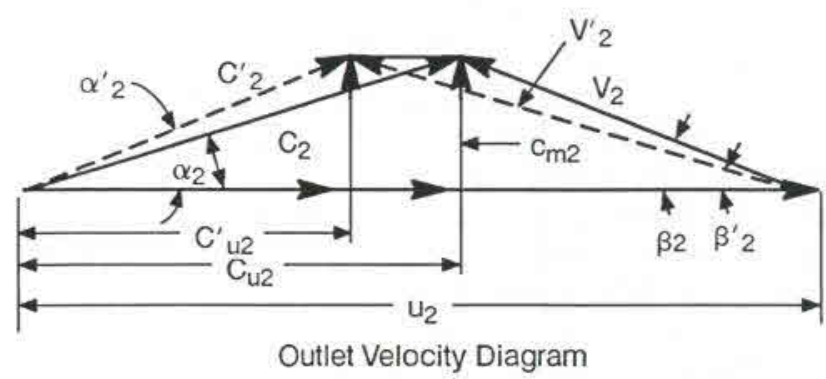

Fig. 6-36 Flow-velocity diagrams for the impeller shown in Fig. 6-35 (drawn in a plane normal to the impeller axis).

to centrifugal forces and to a decrease of flow velocity relative to the impeller, $\mathrm{ft}$

$\Delta \mathrm{H}_{\mathrm{i}}=$ ideal total-pressure-head rise of the fluid flowing through the impeller $=$ the ideal developed head of the pump impeller, $\mathrm{ft}$

$\mathrm{Q}_{\mathrm{imp}}=$ impeller flow rate at the design point (rated conditions), gpm

$A_{1}, A_{2}=$ area normal to the meridional flow at the impeller inlet and outlet, $\mathrm{ft}^{2}$

$\mathrm{d}_{1}, \mathrm{~d}_{2}=$ blade diameter at the impeller inlet and outlet, in.

$\mathrm{u}_{1}, \mathrm{u}_{2}=$ impeller peripheral velocity at inlet and outlet, $\mathrm{ft} / \mathrm{s}$

$\mathrm{v}_{1}, \mathrm{v}_{2}=$ flow velocity relative to the impeller at the inlet and outlet, $\mathrm{ft} / \mathrm{s}$

$\mathrm{c}_{1}, \mathrm{c}_{2}=$ absolute velocity of the flow at the inlet and outlet, $\mathrm{ft} / \mathrm{s}$

$\mathrm{cu}_{1}, \mathrm{cu}_{2}=$ tangential component of the absolute velocity at the inlet and outlet, $\mathrm{ft} / \mathrm{s}$

$\mathrm{cm}_{1}, \mathrm{~cm}_{2}=$ "meridional" or (by definition for radial-flow impellers) radial component of the absolute inlet flow velocity at the inlet and outlet, $\mathrm{ft} / \mathrm{s}$

$\beta_{1}, \beta_{2}=$ impeller blade angle at the inlet and outlet, deg

For centrifugal pumps of the noninducer type (now rarely used in rocket pumps), proper selection of the impeller inlet vane-angle $\beta_{1}$ or the provision of guide vanes at the inlet minimizes the absolute tangential component of fluid flow at the inlet $\mathrm{cu}_{1}$, which for best efficiency should be zero. This is defined as no prerotation, where $\alpha_{1}=90 \mathrm{deg}$. Thus, Eq. (6-24) becomes-

$$
\Delta \mathrm{H}_{\mathrm{i}}=\mathrm{u}_{2} \mathrm{Cu}_{2} / \mathrm{g}
$$


This discussion has assumed ideal conditions. For most rocket applications, centrifugal pumps are designed with an inducer upstream of and in series with the impeller. The flow conditions at the impeller inlet thus are affected by the inducer's discharge flow pattern. In addition, two types of flow usually take place simultaneously in the flow channels, namely, the main flow through the passages and local circulatory (vortex) flows. It is preferable to keep these secondary flows small, but they are always strong enough to significantly affect the overall flow field. As a consequence, the fluid is caused to leave the impeller at an angle $\beta_{2}$ ' less than the impeller discharge vane angle $\beta_{2}$ and to increase the absolute angle $\alpha_{2}$ to $\alpha_{2}{ }^{\prime}$. This and the hydraulic losses in the impeller correspondingly change the relative flow velocities $\mathrm{v}_{1}$ and $\mathrm{v}_{2}$ to $\mathrm{v}_{1}{ }^{\prime}$ and $\mathrm{v}_{2}{ }^{\prime}$, the absolute flow velocities $c_{1}$ and $c_{2}$ to $c_{1}{ }^{\prime}$ and $c_{2}{ }^{\prime}$, and the absolute tangential components $\mathrm{cu}_{1}$ and $\mathrm{cu}_{2}$ to $\mathrm{cu}_{1}{ }^{\prime}$ and $\mathrm{cu}_{2}{ }^{\prime}$. Since the radial-flow areas $\mathrm{A}_{1}$ and $\mathrm{A}_{2}$, and the impeller flow rate $Q_{i m p}$ remain constant, the absolute radial or meridional components $\mathrm{cm}_{1}$ and $\mathrm{cm}_{2}$ also remain unchanged. The inlet- and outlet-flow velocity diagrams in Fig. 6-36 may now be redrawn as represented by the dotted lines. The correlation established in Eq. (6-24) may be rewritten as follows:

$$
\Delta \mathrm{H}_{\mathrm{imp}}=\left(\mathrm{u}_{2} \mathrm{Cu}_{2}{ }^{\prime}-\mathrm{u}_{1} \mathrm{cu}_{1}{ }^{\prime}\right) / \mathrm{g}
$$

where-

$$
\begin{aligned}
\Delta \mathrm{H}_{\mathrm{imp}}= & \text { actual developed head of impeller, } \mathrm{ft} \\
\mathrm{cu}_{1}= & \text { tangential component of the design } \\
& \text { absolute-inlet-flow velocity, } \mathrm{ft} / \mathrm{s} \\
\mathrm{cu}_{2}{ }^{\prime}= & \text { tangential component of the design } \\
& \text { absolute-outlet-flow velocity, } \mathrm{ft} / \mathrm{s}
\end{aligned}
$$

The ratio of the design flow velocity $\mathrm{Cu}_{2}$ ' to the ideal flow velocity $\mathrm{Cu}_{2}$ can be expressed as-

$$
\mathrm{e}_{\mathrm{V}}=\mathrm{cu}_{2}{ }^{\prime} / \mathrm{cu}_{2}
$$

where $\mathrm{e}_{\mathrm{V}}=$ impeller vane coefficient; typical design values range from 0.65 to 0.75 . Referring to Fig. 6-36, Eq. (6-26) may be rewritten:

$$
\mathrm{cu}_{2}^{\prime}=\mathrm{u}_{2}-\mathrm{cm}_{2} /\left(\tan \beta_{2}\right)
$$

By definition, the required impeller-developed head can be determined from-

$$
\Delta \mathrm{H}_{\mathrm{imp}}=\Delta \mathrm{H}+\mathrm{H}_{\mathrm{e}}-\Delta \mathrm{H}_{\text {ind }}
$$

where-

$\Delta \mathrm{H}=$ rated-design pump-developed head, $\mathrm{ft}$

$\Delta \mathrm{H}_{\text {ind }}=$ required inducer head at the rated design point, $\mathrm{ft}$

$\mathrm{H}_{\mathrm{e}}=$ hydraulic head losses in the diffusion system including the volute, ft. Typical design values of $\mathrm{H}_{\mathrm{e}}$ vary from 0.10 to $0.30 \Delta \mathrm{H}$. The required impeller flow rate can be estimated from-

$$
Q_{\text {imp }}=Q+Q_{e}
$$

$$
\begin{aligned}
\text { where- } & \\
\text { Qimp = } & \text { required impeller flow rate at the rated } \\
& \text { design point, gpm } \\
\mathrm{Q}= & \text { rated delivered-pump-flow rate, gpm } \\
\mathrm{Qe}= & \text { impeller leakage losses, gpm. Most of } \\
& \text { these occur at the clearance between } \\
& \text { impeller wearing rings and casing. Typical } \\
& \text { design values of } \mathrm{Qe} \text { vary from } 1 \text { to } 5 \% \text { of } \\
& \text { Qimp. (Oxidizer pumps may be higher.) }
\end{aligned}
$$

\section{Centrifugal-Impeller Design Elements}

After general pump-design parameters, such as developed head $\Delta \mathrm{H}$, capacity $\mathrm{Q}$, suction specific speed $\mathrm{N}_{S S}$, rotating speed $\mathrm{N}$, and specific speed $\mathrm{N}_{\mathrm{S}}$ have been established, a centrifugal (radial) pump impeller may be designed in two basic steps. The first will be selection of velocities and vane angles that are needed to obtain the desired characteristics with optimum efficiency. Usually this can be done with the help of available design or experimental data such as pump-head coefficient $\psi$, impeller vane coefficient $\mathrm{e}_{\mathrm{v}}$, and leakage loss-rate $\mathrm{Q}_{\mathrm{e}}$. The second step, design layout of the impeller for the selected angles and areas, requires considerable experience and skill of the designer to work out the bestperforming configuration based on the given design inputs. This can be done graphically or numerically, but either approach will require a flow analysis to confirm the design using at least a quasi-3D analysis. Codes capable of solving the 3D full Navier-Stokes equations in the complex flow field of a centrifugal impeller are being used but have not yet demonstrated that they are qualified to optimize the design. Three-dimensional Euler codes are used with success in analyzing the flow fields at least near the maximum efficiency point.

The following are considered minimum basic design elements required for proper layout of a radial-flow impeller:

1) The inlet flow coefficient $\phi=\mathrm{cm}_{1} / \mathrm{U}_{\mathrm{e}}$, where $\mathrm{U}_{\mathrm{e}}$ is the velocity of the impeller at the eye. A typical design guideline imposes constant meridional velocity from the inducer discharge to the impeller eye. Thus the inlet flow coefficient is primarily set by the inducer discharge, the hub and tip diameters of the impeller inlet being set to achieve an area equal to, or larger than, that at the inducer discharge. The eye diameter of the impeller frequently is increased relative to the inducer discharge tip-diameter to account for added flow through the wear rings of the impeller. These leakage flows can be relatively large in small pumps, and if the pumped fluid is cryogenic the density of the leakage fluid may be lower, thereby increasing the volumetric flowrate. These leakage flows will be mixing with the core flow from the inducer, but complete mixing will not occur before entering the impeller eye. Thus, the area must be increased to maintain a constant inlet meridional velocity. In the early phases of the design, the leakage flows will not be known but must be estimated based on 
Table 6-5 Allowable impeller tip speeds for different materials.

\begin{tabular}{|l|lllll|}
\hline \multicolumn{7}{|c|}{ Approxmiate tip-speed limit, FT/S } \\
\hline Material & $\begin{array}{l}\text { Forged } \\
\text { Inco 718 }\end{array}$ & $\begin{array}{l}\text { Cast } \\
\text { Inco 718 }\end{array}$ & $\begin{array}{l}\text { Cast } \\
\text { Al 357 }\end{array}$ & $\begin{array}{l}\text { Forged } \\
\text { Ti-5-2.5 }\end{array}$ & $\begin{array}{l}\text { Cast } \\
\text { Ti-5-2.5 }\end{array}$ \\
\hline \multirow{2}{*}{ LOX } & & 760 & N/A & N/A & N/A \\
Hydrogen 1650 & 1400 & 1400 & 1190 & 2000 & 1870 \\
Methane & 1490 & 1260 & 600 & 1240 & 1200 \\
\hline
\end{tabular}

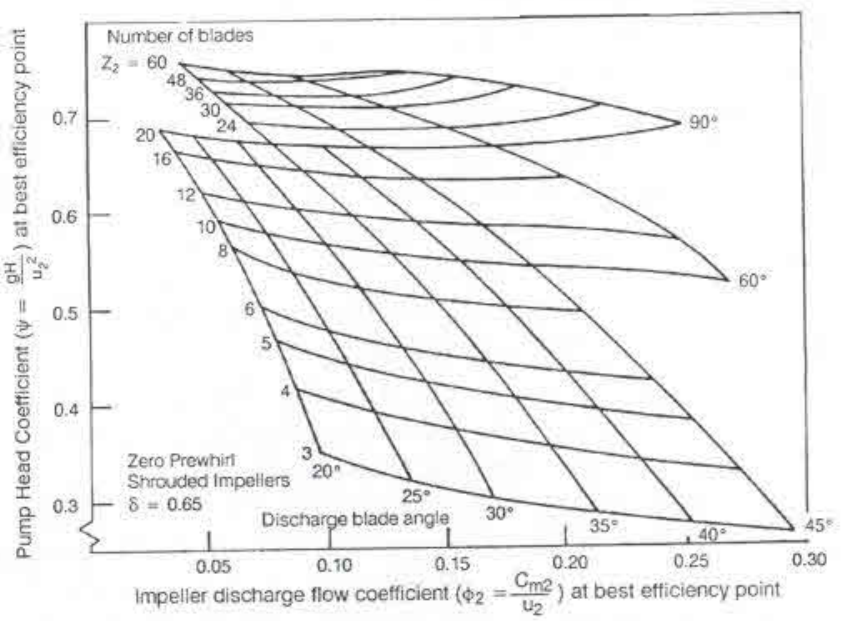

Fig. 6-37 Impeller head coefficient as a function of discharge flow coefficient, blade number, and blade angle.

experience. The flow coefficient for the impellers usually will be higher than for the inducer inlet, but values from 0.08 to 0.4 have been used. Setting this parameter will set the inlet diameters.

The impeller hub diameter must of course be large enough to fit over the shaft. The required shaft diameter can be estimated using the relationships:

$$
\begin{aligned}
& \mathrm{S}_{\mathrm{S}}=16 \mathrm{~T} /\left(\pi \mathrm{d}_{\mathrm{s}} 3\right) \\
& \mathrm{S}_{\mathrm{t}}=32 \mathrm{M} /\left(\pi \mathrm{d}_{\mathrm{s}} 3\right)
\end{aligned}
$$

where-

$\mathrm{d}_{\mathrm{S}}=$ impeller shaft diameter, in.

$\mathrm{T}=$ shaft torque, in. $-\mathrm{lb}$

$\mathrm{M}=$ shaft bending moment, in.-lb.

$\mathrm{S}_{\mathrm{S}}=$ shear stress due to torque, $\mathrm{lb} /$ in $^{2}$

$\mathrm{S}_{\mathrm{t}}=$ tensile stress due to bending moment, $\mathrm{lb} /$ in. $^{2}$

2) The discharge flow coefficient $\phi_{2}=\mathrm{cm}_{2} / \mathrm{U}_{2}$, where $\mathrm{U}_{2}$ is the velocity of the impeller at the tip. The discharge meridional velocity $\mathrm{cm}_{2}$ should be approximately 1.0 to 1.5 times the inlet meridional velocity $\mathrm{cm}_{\mathrm{i}}$. The flow coefficient will then be set by the impeller tip speed selected to meet the head requirements. Values of discharge flow coefficient from 0.05 to 0.3 have been used.

3) Ratio of impeller eye to tip diameters. This ratio should be maintained below 0.70 to avoid efficiency penalties. Larger values have been used, up to 0.8 , but a mixed-flow impeller will usually give a better efficiency for these higher values.

4) The impeller peripheral velocity at the discharge $\mathrm{U}_{2}$. The value of $\mathrm{U}_{2}$ can be calculated by Eq. (6-2) for a given pump-developed head $\mathrm{H}$ and a selected overall pump head coefficient $\psi$. The maximum design value of $\mathrm{U}_{2}$ depends on the material strength, which determines the maximum developed head that can be obtained from a singlestage impeller. Typical maximum values of $\mathrm{U}_{2}$ range from 600 to $2000 \mathrm{ft} / \mathrm{s}$, as shown in Table 6-5. With $\mathrm{U}_{2}$ and $\mathrm{N}$ known, the impeller discharge diameter $\mathrm{d}_{2}$ can be calculated readily.

5) The inlet blade angle $\beta_{1}$. Because the value of $\beta_{1}$ will be affected by the inlet flow conditions, $\beta_{1}$ should ordinarily be made equal or close to the inlet flow angle $\beta_{1}$ ' so that the incidence angle is a small positive value. The incidence may be varied from hub to tip because of the velocity gradients; therefore, it is usually best to select the tip incidence because the tip has the highest relative velocity, which makes it more prone to cavitation. Also, if the pump must operate over a wide flow range, the incidence at the "design point" may be adjusted to enable noncavitating and nonstalled performance over the full required range. Typical design values for $\beta_{1}$ range from 2 to $8 \mathrm{deg}$, with the smaller values at the tip.

6) The discharge blade angle $\beta_{2}$. In the special case of radial-bladed impeller designs, $\beta_{2}$ equals 90 deg. Backward-curved impellers, with $\beta_{2}$ less than 90 $\mathrm{deg}$, are usually more efficient and more stable, and these are used on almost all modern designs. Usually the selection of $\beta_{2}$ is the first step in determining the other impeller design-constants, since most of them depend on $\beta_{2}$. Pump efficiency and head-capacity characteristics are also important considerations for the selection. For a given $\mathrm{U}_{2}$, head and capacity increase with $\beta_{2}$. Figure 6-37 shows the rela- 
tionship between $\beta_{2}$, the discharge flow coefficient $\phi_{2}$, and the number of impeller blades. Typical design values for $\beta_{2}$ range from 17 to 40 deg for backward swept blades.

7) Number of blades. Figure 6-37 has shown the relationship of the number of blades to other key design parameters. The figure shows the minimum number of blades needed to achieve a given head coefficient for an impeller with a ratio of eye-to-tip diameter $(\delta)$ of 0.65 . The number of blades derived from the figure is the number required for the discharge of the impeller. Fewer blades will usually be required at the inlet to avoid cavitation. It is not uncommon, therefore, for a rocket-engine impeller to have partial blades (also known as splitters) which begin partway through the impeller and continue to the exit. Impellers have been successfully used with more than one set of partials; e.g., the SSME HPFTP (Fig. 6-5) has 6 blades at the inlet and 24 blades at the exit. The impeller has a set of long partials and then a set of short partials. The placement of the partial leading edge in the blade passage is critical; it must be chosen by an adequate flow analysis to avoid leading-edge cavitation and to achieve a relatively uniform flow-split. The discharge blade characteristics will usually be identical for full and partial blades, the objective being to achieve a uniform velocity field in each exit passage. As an alternative to Fig. 6-37, the designer can use other published slip factors to calculate the blade angle required to achieve a given fluid angle. (The slip factor is a ratio of the discharge fluid's tangential velocity compared to the ideal velocity obtained if the flow followed the blade.)

8) The width of the impeller at the exit can be calculated by the following correlation:

$$
\beta_{2}=Q_{\text {imp }} /\left(3.12 \pi \mathrm{d}_{2} \mathrm{~cm}_{2} \varepsilon_{2}\right)
$$

where-

$\beta_{2}=$ impeller width at the discharge, in.

$\varepsilon_{2}=$ contraction factor at the discharge. Typical design values range from 0.85 to 0.95 . This nondimensional factor accounts for the blockage due to the blades and the boundary layers. Smaller pumps may require a smaller value.

Qimp = impeller flowrate at the rated design point, gpm

After the blade angles and other dimensions at inlet and discharge have been established, no set rule guides designing the backward-curved blades. The blade profile must be smooth and avoid steep gradients along the suction surface to avoid boundary-layer separation. The blade shape is typically generated using smooth profiles, and then analyzed using an appropriate code (quasi-3D or better). The blade will then be adjusted to attain a smooth variation of velocity from inlet to discharge, with no local areas of large diffusion on the suction surface and no potential for cavitation along the blade. It is also desirable to minimize the length of the passage to reduce friction losses, but this must be balanced by the requirement to control the diffusion rate. The flow-passage shape should be as close to square as possible. It is also desirable to have the blade at the exit be at a relatively constant angular location from tip to hub shroud to avoid corner angles that can increase boundary-layer blockage. The blades should be as thin as the material strength and manufacturing processes will permit. They may be of constant thickness, but the leading-edge profile will be contoured to avoid cavitation and reduce incidence losses. This leading-edge contour may require an appropriate ellipse rather than just a simple circular arc.

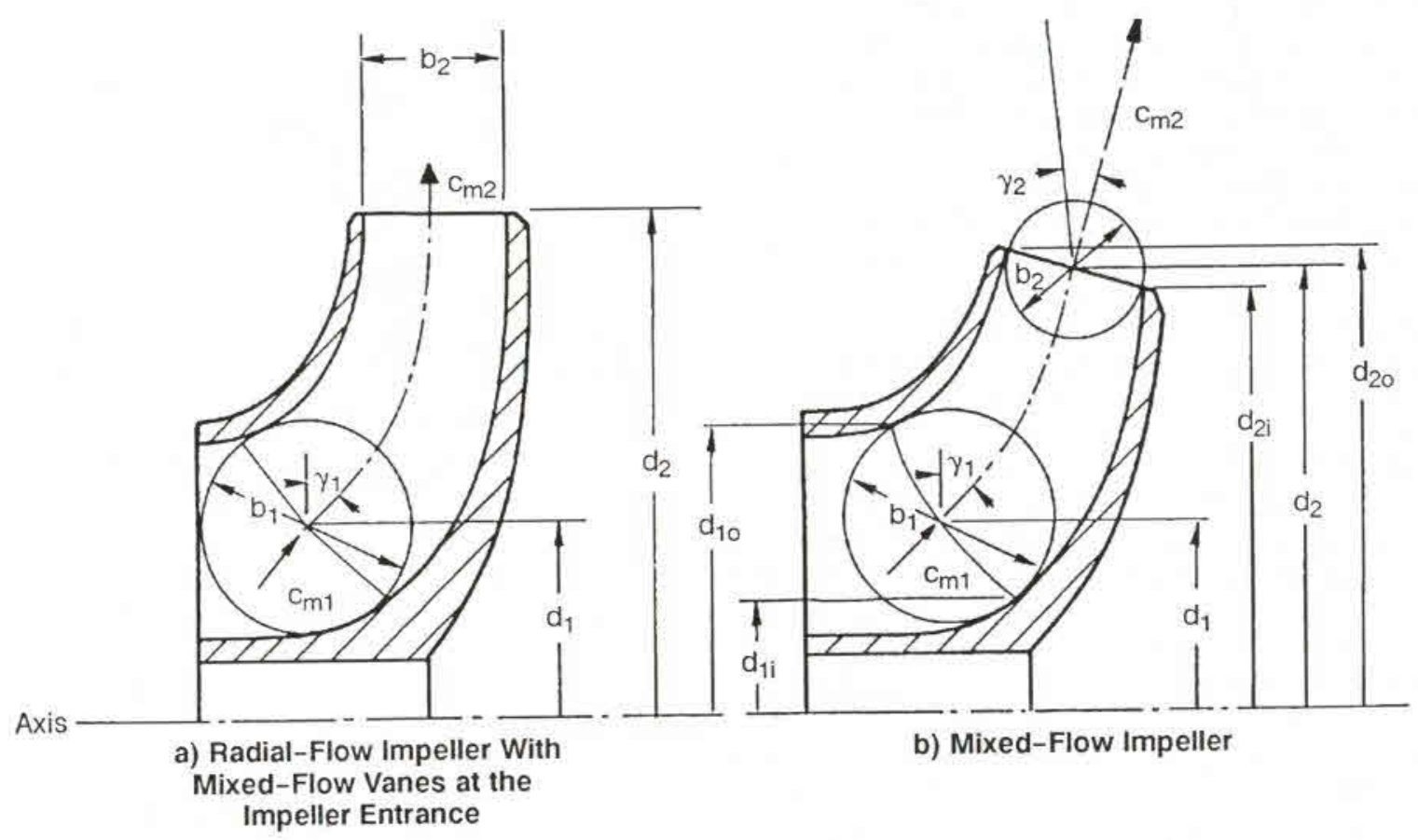

Fig. 6-38 Imneller designs. 
Mixed-flow-type vanes that extend into the impeller entrance or eye (shown in Fig. 6-38a) are frequently used in rocket-engine centrifugal pumps. This is done to match the impeller-inlet flow path with the inducer-discharge flow pattern, to provide more efficient turning of the flow, and to provide structural support to the shrouds.

The mixed-flow-type impeller (shown in Fig. 6$38 \mathrm{~b})$ is used when the impeller needs a large ratio of eye-to-tip diameter. The velocity correlations and design constants of a mixed-flow impeller are essentially the same as those of a radial-flow impeller. Mean effective impeller diameters are used in the calculations for head rise, flow velocities, etc.

\section{Design of Casings}

The pump casing must have the structural integrity to carry loads from the pump to the engine-support system. Internally it must provide hydrodynamic passages efficiently to collect the high-velocity fluid exiting the impeller and convert this kinetic energy into pressure while minimizing the imposed radial load on the rotating members. It does not contribute to the generation of head, but a poor design can significantly reduce both head and efficiency. The construction of a typical centrifugal-pump casing is shown in Fig. 6-4. The front section of the casing, which provides the pump inlet and houses the inducer, is called the suction nozzle. The rear section of the casing, which collects the fluid from the impeller and converts the velocity head into pressure before the discharge, is called the volute. There may be an additional set of stationary vanes called a vaned diffuser between the impeller and the volute.

Since the flow path in a suction nozzle is short and the flow velocities are relatively low, the head loss in a suction nozzle due to friction is very small. The contour of the suction nozzle is designed to suit the inducer configuration. A tapered suction nozzle (as shown in Fig. 6-16) has the area gradually decreasing toward the impeller eye, which steadies the flow and ensures uniform feed to the impeller. However, many pumps cannot efficiently use a tapered inducer because of the need for axial motion of the shaft to balance axial loads. In liquidoxygen pumps, frequently a liner made of a material such as Kel-F or silver is inserted between the inducer and suction-nozzle wall. This eliminates the possibility of metal-to-metal rubbing in the presence of narrow inducer-tip clearances. Rubbing in liquidoxygen pumps may cause dangerous explosions.

Pump inlets are not always configured to permit an axial inlet flow. In such cases the inlet must be designed to provide a relatively uniform inletvelocity field at the inducer inlet and to minimize the losses which reduce the available NPSH for the inducer. These design requirements can be met by using either vaned elbows, a volute scroll with vanes (Fig. 6-5), or a manifold inlet that enters radially on one side and divides the flow into two circumferential flow paths wrapped around the pump and feeding radially inward. Vanes are used in all of these designs to preserve a uniform velocity field at the inducer inlet.
Two basic types of discharge casing are used in rocket centrifugal pumps: the plain volute and the vaned-diffuser volute (see Fig. 6-39). In the first, the impeller discharges into a volute channel of gradually increasing area. Here, the major part of the conversion of velocity to pressure takes place in the conical pump-discharge nozzle at the volute exit. In the latter, the impeller first discharges into a diffuser provided with vanes. A major portion of the diffusion takes place in the channels between the diffusing vanes before the fluid reaches the volute channel. The main advantage of the plain volute is its simplicity. However, the vaned diffuser plus volute can usually provide a higher degree of point efficiency, particularly in high-head pumps. Head losses in pump volutes are relatively high. Approximately $70-90 \%$ of the flow kinetic energy is converted into pressure head in either volute type.

The hydraulic characteristics of a plain volute are determined by several design parameters, such as volute throat area $a_{v}$ and flow areas $a_{\theta}$, included angle $\theta_{s}$ between volute side walls (Fig. 6-40), volute tongue angle $\alpha_{\mathrm{v}}$, radius $\mathrm{r}_{\mathrm{t}}$ at which the volute tongue starts, and volute width $b_{3}$. Pump specific speed Ns somewhat influences the design values. All of the

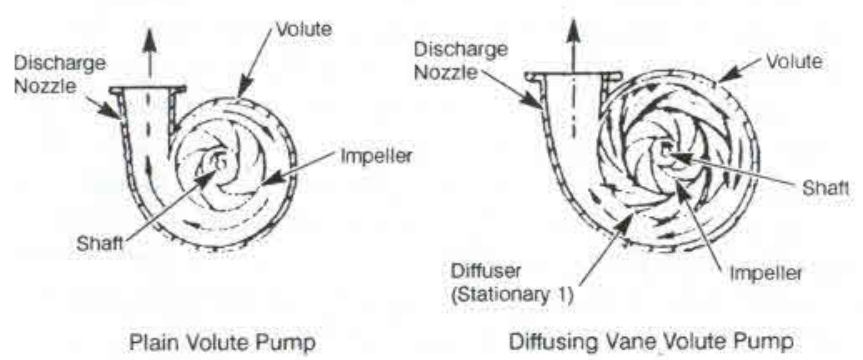

Fig. 6-39 Plain-volute and vaned-diffuser-volute centrifugal pump casings.

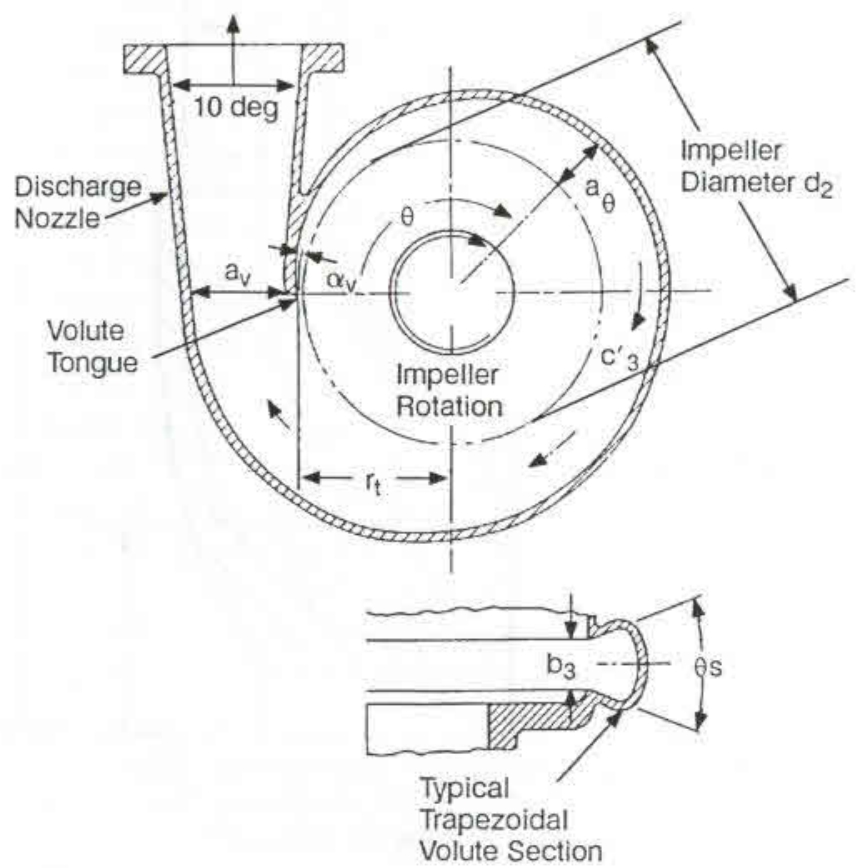

Fig. 6-40 Plain volute casing of a centrifugal pump. 
pump flow $Q$ passes through the volute throat section $a_{v}$, but only part of it passes through any other section; the amount depending on the location away from the volute tongue.

As its primary design requirement, the volute must provide a uniform pressure boundary at the impeller outer diameter at least at the design point. That is, the single discharge introduces an asymmetry to the pump that at off-design conditions produces a pressure gradient at the impeller discharge, causing a radial load acting on the rotating member that must be carried by the bearings. At the design point of the volute, these radial loads are theoretically zero. This design condition approximates a linear area distribution with circumferential angle, but is not exactly linear for two reasons. First, the friction forces acting on the volute walls affect the velocity/pressure field. Second, the decrease in velocity because of the change in radius (conservation of angular momentum) must be included.

To avoid impact shocks and separation losses at the volute tongue, the volute angle $\alpha_{\mathrm{v}}$ is designed to correspond to the direction of the absolute velocity vector at the impeller discharge. The radius $\mathrm{r}_{\mathrm{t}}$ at which the tongue starts should be $5-10 \%$ larger than the outside radius of the impeller to minimize dynamic loads on the impeller blades and the volute tongue.

The dimension $b_{3}$ at the bottom of a trapezoidal volute cross-section will be chosen to minimize losses from friction between impeller discharge flow and volute side-walls. The width $b_{3}$ is generally set between 1.0 and 1.25 times the impeller width at the discharge for pumps without a vaned diffuser; lowspecific-speed pumps could be even higher. The shape of the volute shown in Fig. 6-40 results in a symmetric design that will generate a double vortex, as shown in Fig. 6-41. An asymmetric cross section is preferred because it produces a single vortex that is more stable and improves overall efficiency. For designs with diameter limits, a folded volute can be used (also shown in Fig. 6-41). For the symmetrical volute, the maximum included angle $\theta_{\mathrm{S}}$ between the volute sidewalls should be about $60 \mathrm{deg}$. For higherspecific-speed pumps or for higher impeller-discharge flow angles, the value of $\theta_{\mathrm{S}}$ should be made smaller.

The pressure at the outer diameter of the impeller cannot always be kept uniform, especially under off-design operating conditions. This results in a radial thrust on the impeller shaft. To eliminate or reduce the radial thrust, both double-tongue and double-discharge volute designs have been used (Fig. 6-42). Here, the flow is divided into equal streams by two (or more) tongues set $180 \mathrm{deg}$ apart. Although the volute pressure unbalances may be the same as in a single-volute casing, the resultant of all radial forces may be reduced to a reasonably low value because of symmetry.

Vaned diffusers have also been used extensively, particularly in higher-pressure pumps. The diffuser vanes serve as structural members to maintain the integrity of the volute under hoop stresses induced

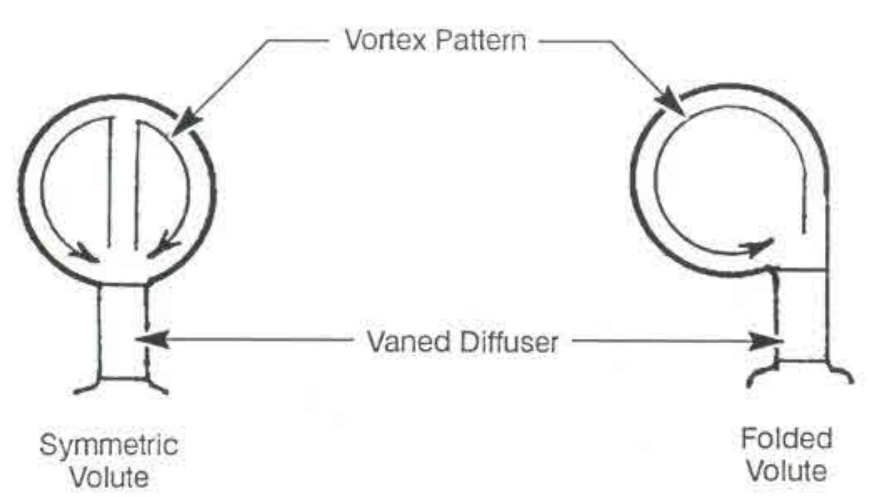

Fig. 6-41 Potential volute configurations.
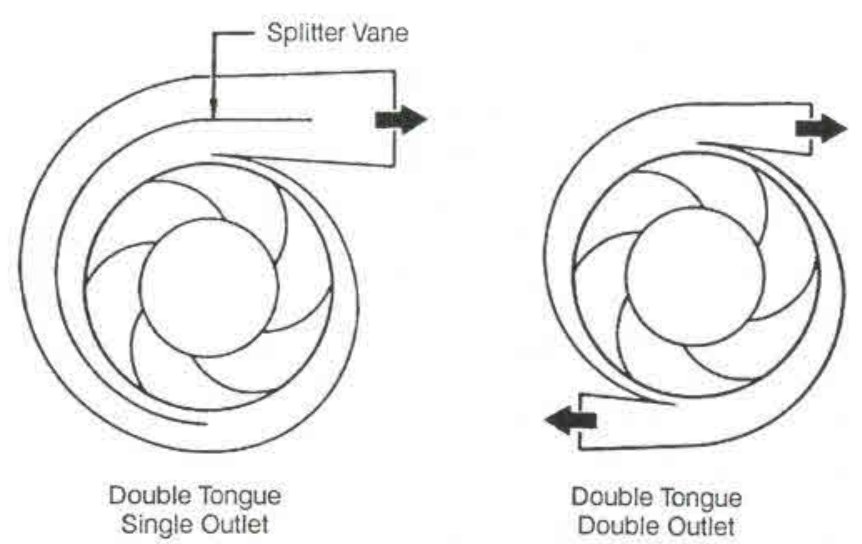

Fig. 6-42 Typical double-tongue and doubledischarge volute configurations.

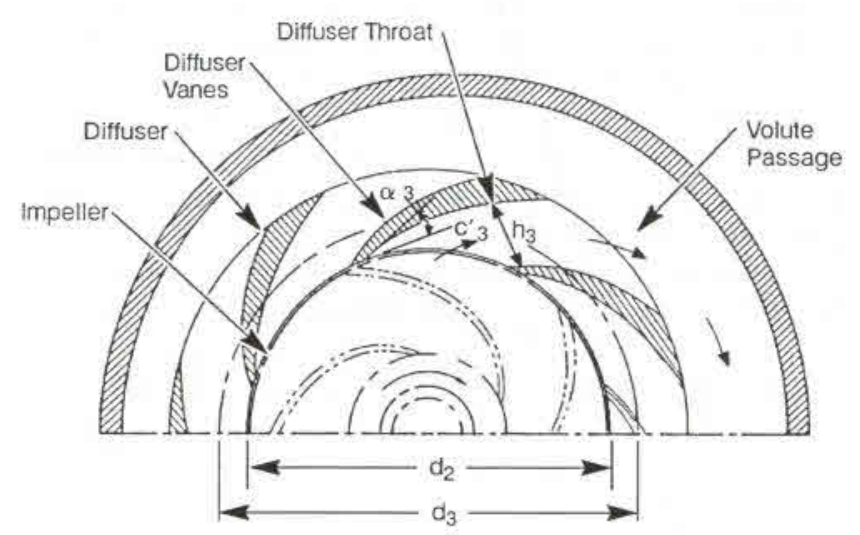

Fig. 6-43 Typical layout of the diffuser for a pump.

by internal pressures. Also, they are sometimes used as the primary path for structural loads carried through the housing. Hydrodynamically, the use of the diffuser improves efficiency, at least near the design point and tends to reduce radial loads at the off-design conditions by significantly reducing the velocity in the volute and maintaining a more nearly constant velocity angle. Figure 6-43 shows a vane-island diffuser that is desirable when the diffuser is in the structural-load path. Diffuser vanes with aerodynamically shaped discharges as well as inlets are used for peak efficiencies where the vanes do not 
have to carry large loads. The radial clearance between impeller and diffuser-inlet vane tips must be sufficiently large to avoid large dynamic stresses on the impeller blades and diffuser vanes because of dynamic interaction. The preferred ratio of diffuserinlet to impeller-discharge diameter is in the range of 1.07 to 1.10 . However, for peak efficiencies and to reduce size and weight, smaller values are sometimes used, e.g., 1.05, particularly in low-density fluids such as liquid hydrogen. The width of the diffuser at its inlet should be similar to or up to $10 \%$ smaller than the width of the impeller's discharge flow.

The design of the vane shapes requires careful flow analysis to achieve efficiency and avoid inlet stall or cavitation. Typically, the design-point incidence angle of the inlet will be kept small unless the desired operating flow range allows the design be biased in one direction to minimize stall over the whole range. At the low-flow condition it is not uncommon for the vaned diffuser inlet to be the first location to experience stall, although the head loss due to the stall may be relatively small (e.g., less than $5 \%$ of the overall head).

The number of diffuser vanes (z) should be kept to the minimum, consistent with good performance, and to avoid resonances should have no common factor with the number of impeller vanes. If possible, the cross section of the passages in the diffuser should be made nearly square (i.e., $b_{3}=h_{3}$ ). The shape of the passage below the throat should be diverging, with an angle between 10 and $12 \mathrm{deg}$. The flow leaving the diffuser should have velocity kept slightly higher than the velocity in the pumpdischarge line.

Multistage centrifugal pumps require crossover channels to direct the flow from the discharge of one stage to inlet of the next. Crossovers can be external using a volute collector at the discharge of one stage and a duct routed to the inlet manifold of the next. However, this leads to a much larger, heavier, more-costly design as compared with an internal-crossover system.

Internal-crossover design involves conversion of kinetic energy into pressure, turning the flow 180 deg to the inlet of the next stage, and distributing the flow uniformly at the inlet to the next impeller. Crossover requirements can best be met in separate steps. The continuous-passage crossover (as found in the SSME HPFTP, Fig. 6-5) is a good example. The majority of the pressure recovery is achieved in the upcomer, which is a passage diffuser with design features similar to a vaned diffuser's. Flow will be turned in a constant-area turnaround duct, designed to minimize secondary flows and their associated losses. The remaining pressure recovery takes place in the downcomer, which is also designed to provide the desired inlet velocity vector at the next stage inlet. The flow is discharged from the downcomer in an annular channel long enough to provide some mixing of the wakes and boundary-layer growth developed in the crossover passage. The flow then enters the eye of the next impeller.

\section{Balancing the Axial Thrust of Centrifugal Pumps}

The pressures generated in these pumps and the areas of the rotating members are sufficiently large that the designer must hydrodynamically balance the axial loads acting on the rotating components. This balance must be sufficient to maintain low axial loads on the bearings throughout operation-for transient operation as well as steady state. If the pump is required to throttle, the balance must be adequate throughout the throttle range to protect the bearings from overload. Various techniques are available to achieve a balance design. For the turbopump in Fig. 6-16, the two impellers are mounted back-to-back with balance ribs on the back of the impeller that can be adjusted to achieve a reasonable balance at a fixed operating condition. The discharge pressures attained in this pump and the single-point operation yielded an acceptable design, requiring no self-adjusting control system.

The pressure forces acting on the surface of the rotating members are strongly affected by the magnitude of the rotational velocity of the fluid in the cavity between the rotating and stationary walls.

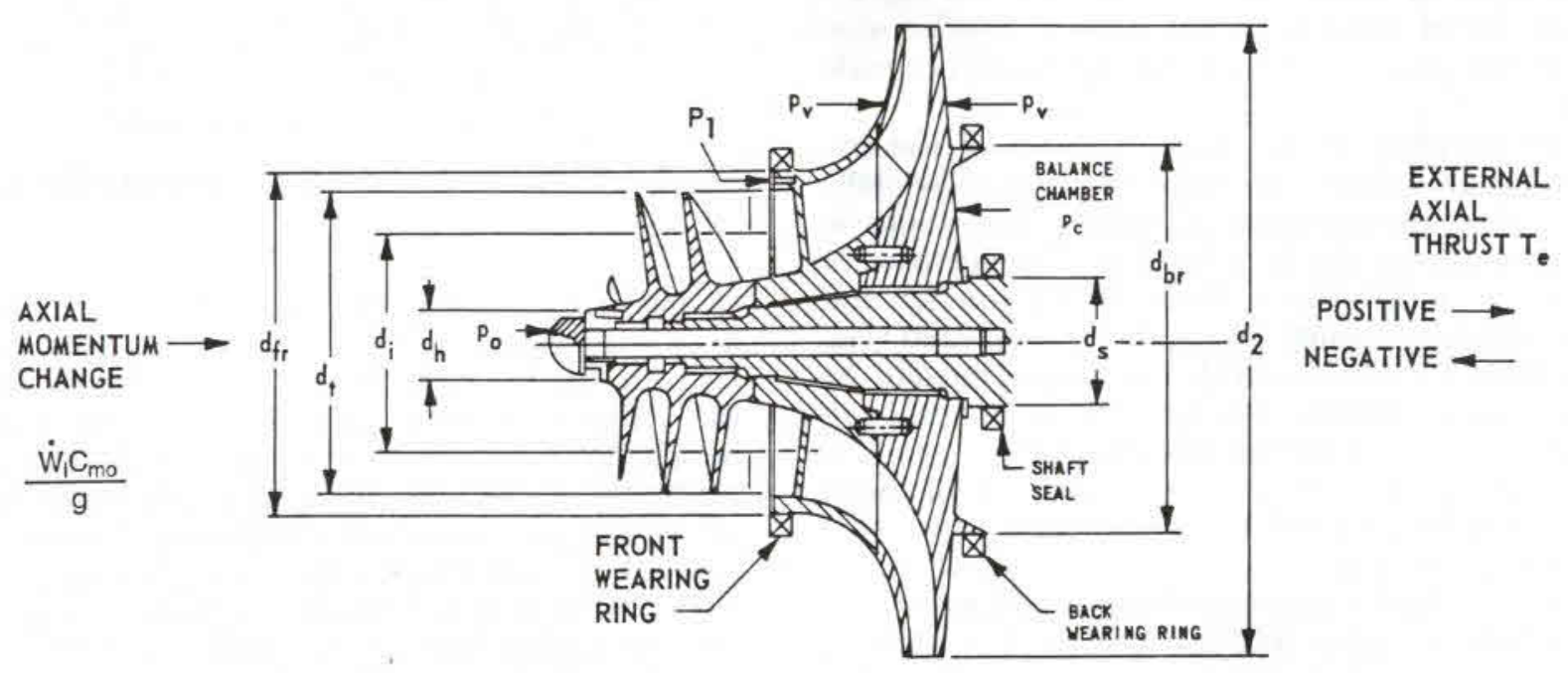

Fig. 6-44 Balancing axial thrusts of a centrifugal pump by the balance-chamber method. 
Frequently, the velocity in the cavity can be approximated by a forced-vortex type of flow (velocity linearly dependent on radius). With such an approximation, pressure at any point along the rotating surface is given by-

$$
\mathrm{p}_{\mathrm{r}}=\mathrm{p}_{1}-\rho \mathrm{k}^{2}\left(\mathrm{U}_{1}^{2}-\mathrm{U}_{\mathrm{r}}^{2}\right) / 2 \mathrm{~g}
$$

where $p_{1}, p_{r}$ are the pressures at the tip and at any other radius, respectively; $\mathrm{U}_{1}$ and $\mathrm{U}_{\mathrm{r}}$ are the corresponding rotational speeds of the wheel; $\rho$ is the fluid specific weight, and $g$ is the gravitational constant; $\mathrm{k}$ is the ratio of the average fluid tangential velocity $\mathrm{c}$ divided by the rotational velocity of the wheel $\mathrm{U}$ at any radius. Note that if $\mathrm{k}$ is large, the pressure gradient is large, and the pressure force is less as the diameter decreases. Placing balance ribs on the rotating wheel increases the fluid velocity; that produces a higher $\mathrm{k}$ value and reduces the force. As another option, placing antivortex ribs on the stationary surface tends to decrease the $\mathrm{k}$ value and increase the fluid force. For two smooth surfaces with no appreciable through-flow, the average value of $\mathrm{k}$ will be 0.5 .

Rather than ribs, seals can be used to achieve a balance, as illustrated in Fig. 6-44. A balance chamber is provided at the back shroud of the impeller, between back wearing-ring diameter $\mathrm{d}_{\mathrm{br}}$ and shaft-seal diameter $d_{S}$. Proper selection of the projected chamber area and of the admitted fluid pressure balances axial loads. The pressure level pc in a balance chamber can be controlled by careful adjustment of the clearances and leakages of the back wearing ring and the shaft seals. The required $\mathrm{p}_{\mathrm{c}}$ can be determined by integrating the pressure forces over the respective areas to achieve the necessary balance.

The high-pressure pumps on the SSME operate over a throttle range and involve such large pressures that more sophisticated balancing techniques were required. To illustrate the techniques available to the designer, consider the SSME HPFTP of Fig. 6-5. The pump and turbine being on the same shaft, both must be included in the axial-thrust model. The turbine thrust was designed to oppose the pump thrust to help achieve an overall balance. The labyrinth seals on the back side of the impellers
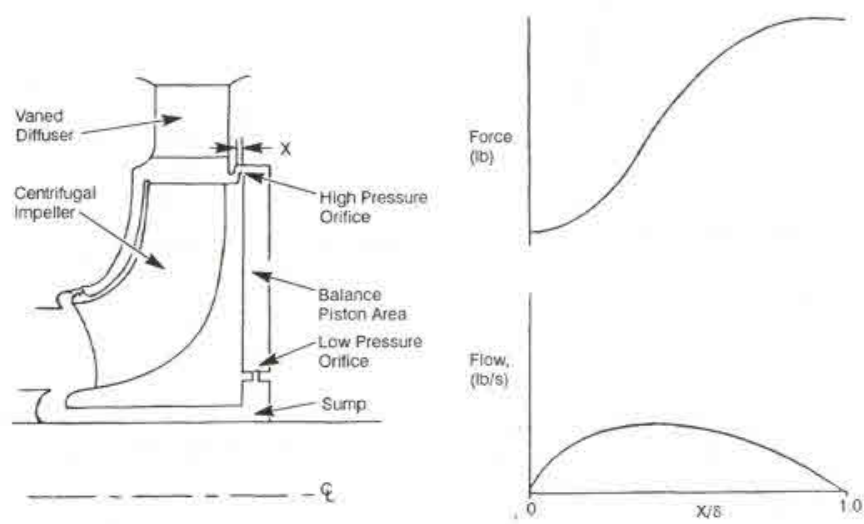

Fig. 6-45 Balance-piston concept. were given a diameter that would improve overall balance. Finally, to provide some self-adjusting ability, a balance piston (Fig. 6-45) was incorporated on the back side of the third-stage impeller. The complete rotating assembly is free to move over a limited range, in the axial direction, to respond to any unbalanced loads. As the assembly moves it opens one of the two orifices of the balance piston and closes the other. This adjusts the pressure on the back side of the impeller that changes until arriving at a balanced force. The total available travel is typically small (e.g., 0.020 in.), and the balance piston is designed to operate at the middle of the force range at the operating point with the highest speed.

\subsection{DESIGN OF AXIAL-FLOW PUMPS}

Except when used as inducers, axial-flow pumps for rocket engines have essentially been limited to liquid-hydrogen systems in a multistage configuration where the required flow range of operation was limited (Ref. 6-7). Multistage axial-flow hydrogen pumps are used in applications going beyond the capability of a single-stage centrifugal pump, since the multistage construction is comparatively simple (Fig. 6-3). As shown in Fig. 6-6, the fluid in an axialflow pump flows from one stage to the next with a minimum of connecting passages.

The head coefficient of a typical single-stage centrifugal hydrogen pump falls in the range of 0.45 0.65 . For an axial pump, however, the head coefficient for a single stage will usually be limited to the range of $0.2-0.4$. Thus, more stages will be

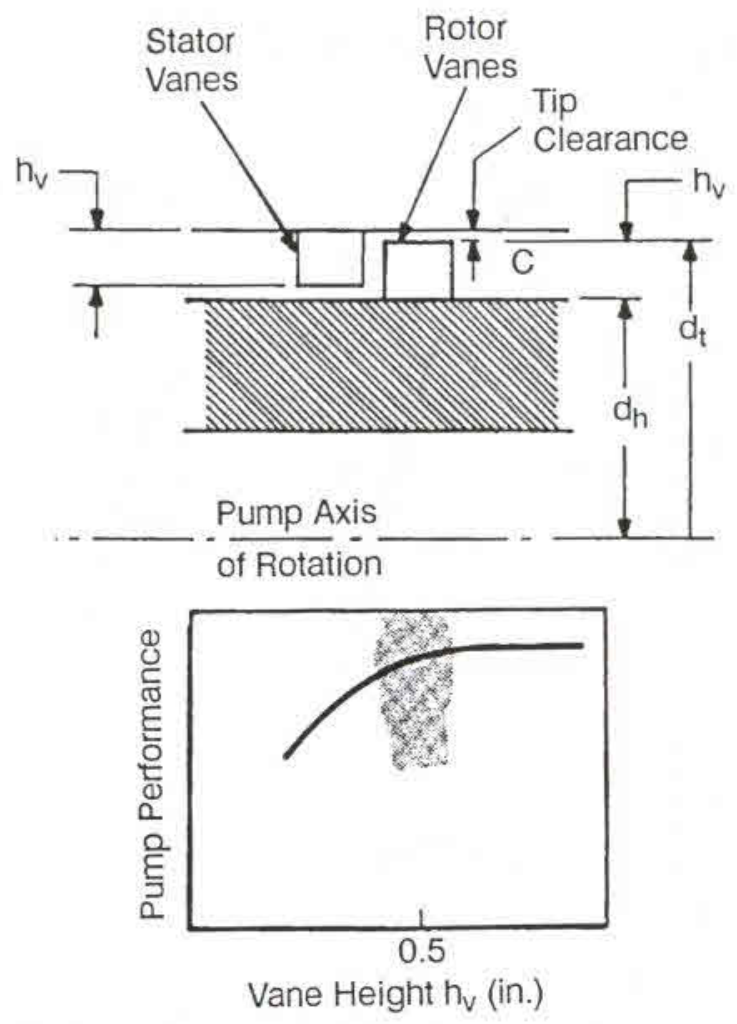

Fig. 6-46 Effect of vane height on the performance of an axial-flow pump. 
required to get the same head for the axial pump, but the staging is much easier to achieve. Typically, the axial pump will have a lower flow-limit because of the minimum practical height $h_{\mathrm{V}}$ of the vanes (Fig. 6-46). For heights below 0.5 in., the tip clearance required for efficient performance becomes critical, causing manufacturing problems or increased risk of operation. Each axial stage should preferably have a flow coefficient in the range of 0.25 or higher and the stage hub/tip diameter ratio should generally be less than 0.9 for efficient operation. Using these guidelines, the designer can parametrically evaluate the relative design features, such as diameter and blade height, to meet requirements. Also, a reduction in rotor diameter below certain values will not be practical because of the high $\mathrm{rpm}$ required for proper blade speed.

Axial pumps typically are used where specific speeds per stage are in the range of 3000 to 6000 . An area of overlap allows either a multistage axial or centrifugal pump. The better solution will be dictated by other considerations, such as space envelope, mounting and ducting arrangement, required flow range, etc. Probably the major limit of axial pumps is their tendency to stall at low-flow conditions. This stall involves separation of the boundary layers on the blades, and it significantly reduces both pump head and efficiency. This can be seen in Fig. 6-47, which presents actual data of a multistage axial flow pump. The pump operation must be maintained at some comfortable margin (e.g., 10\%) of flow above the stall condition.

\section{Basic Assumptions for Axial-Flow Pumps}

During operation of an axial-flow pump, it is assumed that the meridional or axial component $\mathrm{cm}$ of the absolute flow velocity remains constant throughout all stages of the impeller rotor and the stator. This approximation assumes that the thermodynamic conditions do not change sufficiently to require adjusting the density of the fluid in the stages. If the density changes are significant, they should be accounted for at least in the interstage planes. (There is seldom enough density change to require analyzing the pump flow as a compressible fluid in a given stage. However, the pump designer must be aware of the potential for cavitation and design to avoid it.) To satisfy the flow-continuity equation, the cross-sectional areas of the various flow passages at right angles to $\mathrm{cm}$ must also remain constant. This assumption is reasonable, except for the effects of frictional drag at the casing walls and the vanes. Again, where significant boundary layers develop it may be desirable to increase the blade heights in successive stages to maintain an approximately constant $\mathrm{cm}$ in the core flow.

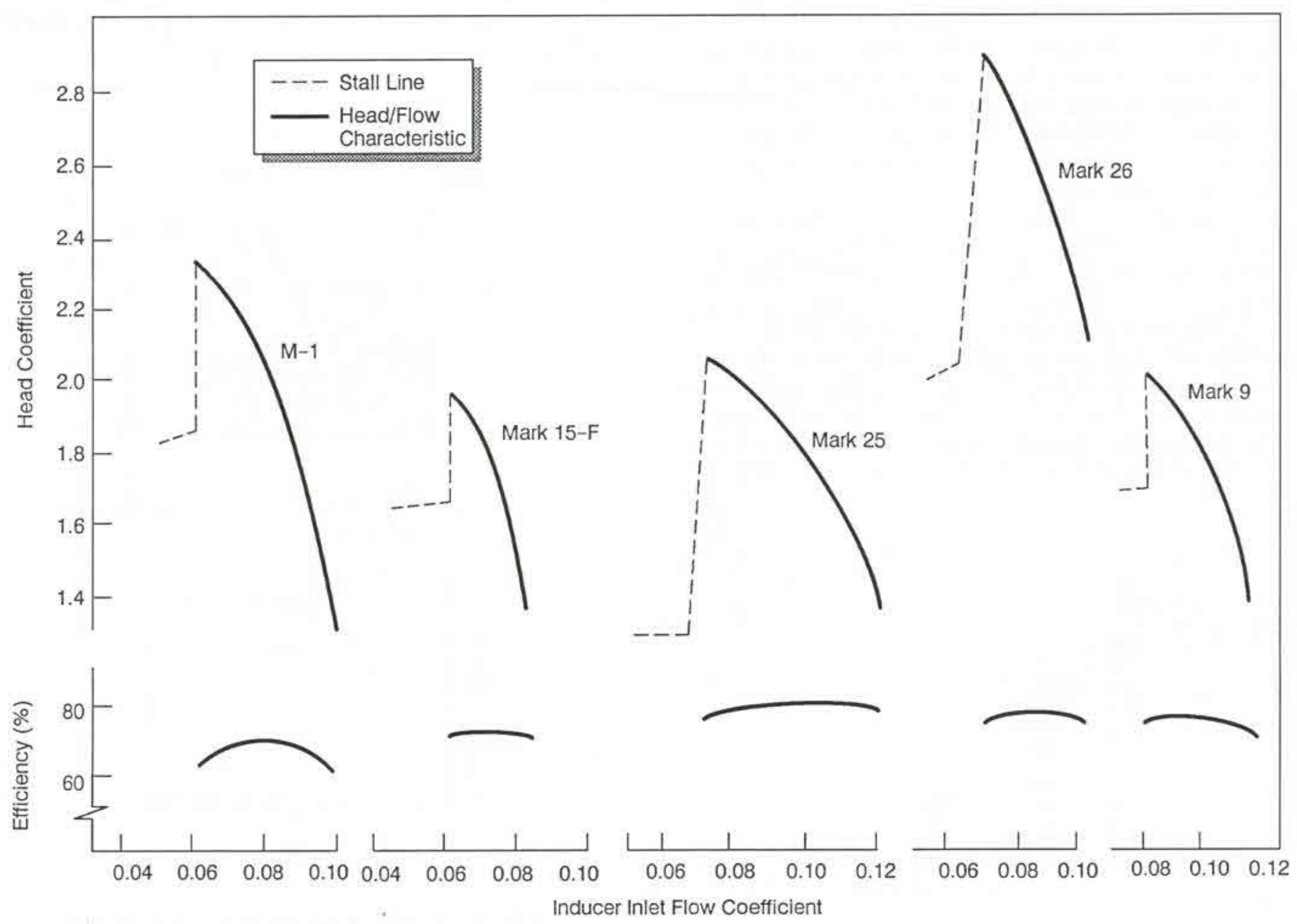

Fig. 6-47 H-Q, $\eta-Q$ data for axial pump with stall. 


\section{Operation of the Impeller Rotor}

As its main function, the impeller rotor of an axialflow pump-by the action of airfoil-shaped rotor vanes (Fig. 6-48 and 6-49-imparts kinetic energy to the fluid by increasing the tangential component of the absolute flow velocity. It is convenient to describe the vanes on several developed cylindrical sections. Three sections are of particular interest: at the impeller tip diameter $d_{t}$, at the impeller hub $d_{h}$, and at its mean effective diameter $\mathrm{d}_{\mathrm{m}}$ (inches). The mean effective diameter is defined by-

$$
\mathrm{d}_{\mathrm{m}}^{2}=0.5\left(\mathrm{~d}_{\mathrm{t}}^{2}+\mathrm{d}_{\mathrm{h}}^{2}\right)=0.5 \mathrm{~d}_{\mathrm{t}}^{2}\left(1+\mathrm{r}_{\mathrm{d}}^{2}\right)
$$

where $r_{d}=$ impeller hub ratio, or $d_{h} / d_{t}$. For simplicity, vane characteristics and flow conditions are discussed here only with respect to the mean effective diameter $d_{m}$. The vanes are equally spaced at a circumferential distance $\mathrm{P}_{\mathrm{r}}$ :

$$
\mathrm{P}_{\mathrm{r}}=\pi \mathrm{d}_{\mathrm{m}} / \mathrm{z}_{\mathrm{r}}
$$

where $-\mathrm{P}_{\mathrm{r}}=$ pitch or rotor vane spacing at the mean effective diameter $\mathrm{d}_{\mathrm{m}}$, in., and $\mathrm{z}_{\mathrm{r}}=$ number of rotor vanes. The ratio of the rotor-vane chord length $C_{T}$ to the pitch $\mathrm{P}_{\mathrm{r}}$ is called rotor-vane solidity $\mathrm{S}_{\mathrm{r}}$ :

$$
\mathrm{S}_{\mathrm{r}}=\mathrm{C}_{\mathrm{r}} / \mathrm{P}_{\mathrm{r}}
$$

where $\mathrm{S}_{\mathrm{r}}=$ rotor-vane solidity at the mean effective diameter $d_{m}$. The solidity generally increases from rotor-tip diameter $d_{t}$ to hub diameter $d_{h}$ for structural reasons.

The profile of the vane can be represented by the vane mean line (Fig. 6-49), which determines most of the important hydraulic properties of the vane. The thickness of the vane varies along the mean line for better performance and for structural strength. To effectively impart the driving action to the fluid, the angle of the vane mean line, or rotorvane angle, is gradually increased from $\beta_{2}$ to $\beta_{3}$. The difference between the two, $\beta_{3}-\beta_{2}$, gives a measure of the vane curvature (camber) along any particular vane section. Mean-line geometries developed for axial compressors and airfoils are generally applicable to pumps, but the potential for leading-edge cavitation must be evaluated and avoided for a good design. In axial-flow pump designs, all vane mean lines can often be approximated by a circular arc. For this case, the following correlations can be established:

$$
\begin{gathered}
\beta_{\mathrm{C}}=0.5\left(\beta_{2}+\beta_{3}\right) \\
\mathrm{C}_{\mathrm{r}}=2 \mathrm{R}_{\mathrm{r}} \sin \left[0.5\left(\beta_{3}-\beta_{2}\right)\right]=\mathrm{L}_{\mathrm{r}} / \sin \beta_{\mathrm{c}}
\end{gathered}
$$

where-

$\beta_{\mathrm{C}}=$ chord angle of the rotor vane, deg

$\beta_{2}=$ vane angle at the rotor inlet, deg

$\beta_{3}=$ vane angle at the rotor outlet, deg

$\mathrm{C}_{\mathrm{r}}=$ chord length of the rotor vane, in .

$\mathrm{R}_{\mathrm{r}}=$ radius of the rotor vane curvature, in.

$\mathrm{L}_{\mathrm{r}}=$ axial length of the rotor vane, in.

An angle of attack (incidence angle) " $\mathrm{i}$ " between rotor-inlet vane angle $\beta_{2}$ and the direction of the relative velocity of the flow entering the rotor $\beta_{2}$ ' allows more effective driving of the fluid. Also, an angle "ii' allows deviation of the flow from the rotoroutlet vane angle $\beta_{3}$, the direction of the relative velocity of the flow leaving the rotor being $\beta_{3}$ '. For the design of impeller rotors, velocity diagrams of the flows at the inlet and outlet of rotor vanes can be constructed (Fig. 6-49) with the following

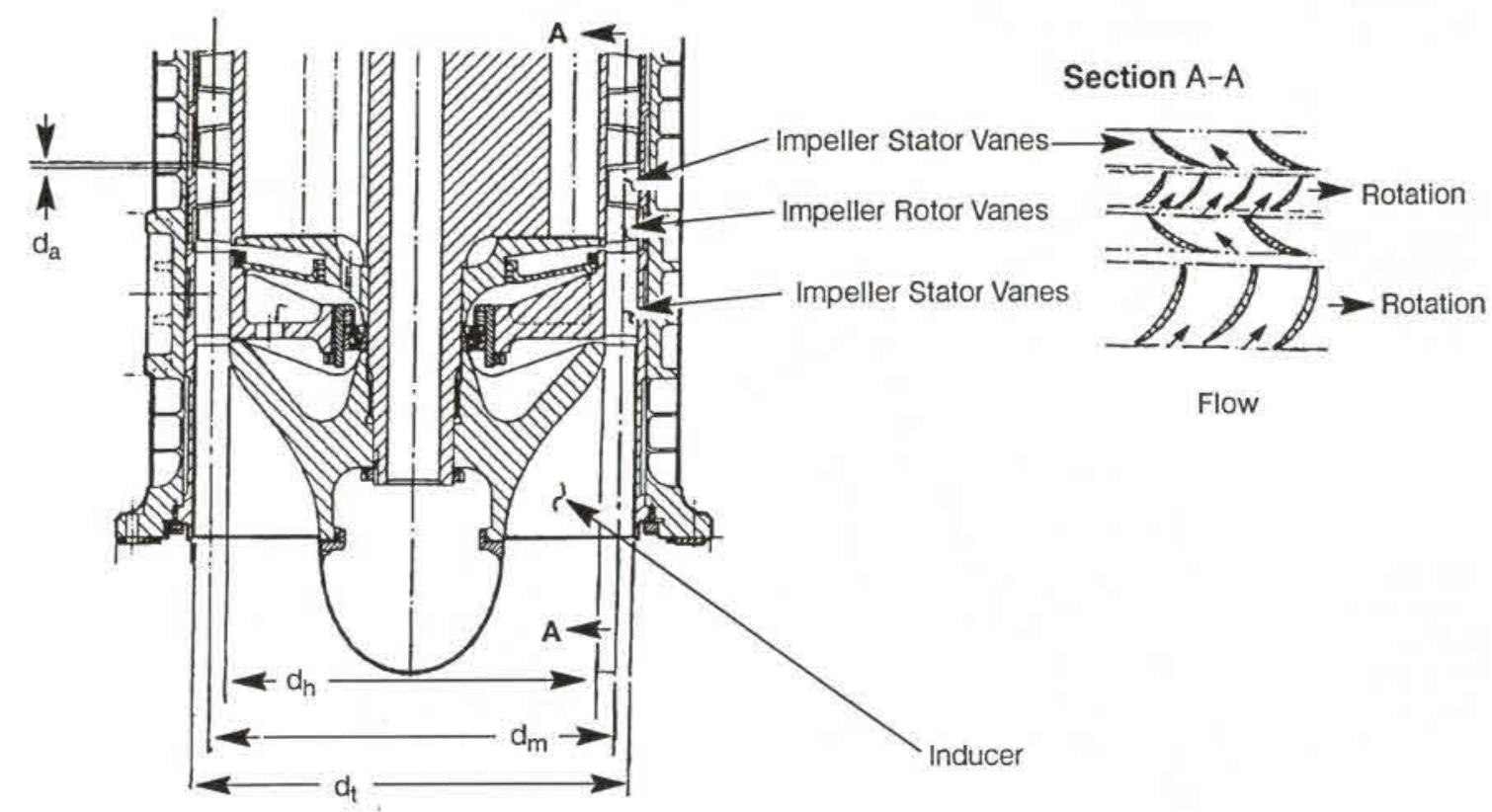

Fig. 6-48 Inducer, inducer stator, impeller rotor, and impeller stator of an axial-flow pump. 
correlations:

$$
\begin{aligned}
& \beta_{2}=\beta_{2}^{\prime}+i \\
& \beta_{3}=\beta_{3^{\prime}}+\mathrm{ii} \\
& c_{m}=Q_{i m p} /\left[3.12 p\left(d_{t}^{2}-d_{h^{2}}\right) \varepsilon / 4\right] \\
& \mathrm{Q}_{\mathrm{imp}}=\mathrm{Q}+\mathrm{Q}_{\mathrm{e}} \\
& \mathrm{u}_{\mathrm{m}}=\pi \mathrm{N} \mathrm{d}_{\mathrm{m}} / 720 \\
& \Delta \mathrm{H}_{\mathrm{imp}}=(\Delta \mathrm{H})_{1}+\mathrm{H}_{\mathrm{e}} \\
& =\left[\mathrm{u}_{\mathrm{m}} \mathrm{c}_{\mathrm{u} 3^{\prime}}-\mathrm{u}_{\mathrm{m}} \mathrm{c}_{\mathrm{u} 2^{\prime}}\right] / \mathrm{g} \\
& (\psi)_{1}=(\Delta \mathrm{H})_{1} / \mathrm{u}^{2} / \mathrm{g} \\
& c_{\mathrm{m}} \quad=\mathrm{c}_{\mathrm{u} 2}{ }^{\prime} \tan \alpha_{2}^{\prime}=\mathrm{c}_{\mathrm{u} 3^{\prime}} \tan \alpha_{3}^{\prime} \\
& =c_{2}{ }^{\prime} \sin \alpha_{2}^{\prime}=c_{3}^{\prime} \sin \alpha_{3}^{\prime} \\
& =\mathrm{v}_{2}^{\prime} \sin \beta_{2}^{\prime}=\mathrm{v}_{3}^{\prime} \sin \beta_{3^{\prime}}
\end{aligned}
$$

where

$i=$ angle of attack, deg

ii = discharge deviation angle, deg

$\beta_{2}^{\prime}, \beta_{3}^{\prime}=$ relative flow angles at the rotor inlet and outlet, deg

$\alpha_{2}^{\prime}, \alpha_{3}^{\prime}=$ absolute flow angles at the rotor inlet and outlet, deg

$\mathrm{cm}=$ meridional or axial component of the absolute flow velocities, $\mathrm{ft} / \mathrm{s}$

$\mathrm{u}_{\mathrm{m}}=$ rotor peripheral velocity at mean effective diameter $\mathrm{dm}, \mathrm{ft} / \mathrm{s}$

$c_{2}^{\prime}, c_{3}^{\prime}=$ design absolute flow velocities at the rotor inlet and outlet, $\mathrm{ft} / \mathrm{s}$

$\mathrm{cu}_{2}^{\prime}, \mathrm{cu}_{3}^{\prime}{ }^{\prime}=$ tangential components of the design absolute rotor inlet and outlet flow velocities, $\mathrm{ft} / \mathrm{s}$

$\mathrm{v}_{2}{ }^{\prime}, \mathrm{v}_{3}{ }^{\prime}=$ design relative flow velocities at the rotor inlet and outlet, $\mathrm{ft} / \mathrm{s}$

$$
\begin{aligned}
Q_{\text {imp }}= & \text { required impeller flowrate at the } \\
& \text { rated design point, gpm } \\
\mathrm{Q}= & \text { rated design pump flowrate, gpm } \\
\mathrm{Qe}_{\mathrm{e}}= & \text { impeller-leakage loss rate }(2 \text { to } 10 \% \\
& \text { of } \mathrm{Q}) \text {, gpm } \\
\varepsilon= & \text { contraction factor of vane passage } \\
& (0.85 \text { to } 0.95) \\
\mathrm{H}_{\mathrm{imp}}= & \text { developed head required per } \\
& \text { impeller stage, } \mathrm{ft} \\
(\Delta)_{1}= & \text { rated design developed head per } \\
& \text { axial-flow-pump stage, } \mathrm{ft} \\
\mathrm{H}_{\mathrm{e}}= & \text { hydraulic-head losses per stage of } \\
& \text { impeller stator, } \mathrm{ft} \\
(\psi)_{1}= & \text { head coefficient per axial-flow pump } \\
& \text { stage }
\end{aligned}
$$

At various cylindrical sections between vane tip diameter $d_{t}$ and hub diameter $d_{h}$, the following correlations between vane angles and flow velocities have been established:

$d_{m} \tan \beta_{2}=d_{t} \tan \beta_{2 t}=d_{h} \tan \beta_{2 h}=d_{x} \tan \beta_{2 x}$

$d_{m} \tan \beta_{3}=d_{t} \tan \beta_{3 t}=d_{h} \tan \beta_{3 h}=d_{x} \tan \beta_{3 x}$

$\mathrm{u}_{\mathrm{m}} / \mathrm{d}_{\mathrm{m}} \quad=\mathrm{u}_{\mathrm{t}} / \mathrm{d}_{\mathrm{t}}=\mathrm{u}_{\mathrm{h}} / \mathrm{h}=\mathrm{u}_{\mathrm{x}} / \mathrm{d}_{\mathrm{x}}$

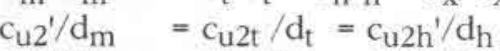

$\mathrm{c}_{\mathrm{u} 3^{1}} / \mathrm{d}_{\mathrm{m}}=\mathrm{c}_{\mathrm{u} 3 \mathrm{t}^{1}} / \mathrm{d}_{\mathrm{t}}=\mathrm{c}_{\mathrm{u} 3 \mathrm{~h}^{1}} / \mathrm{d}_{\mathrm{h}}$

where-

$\beta_{2 \mathrm{t}}, \beta_{2 \mathrm{~h}}=$ rotor-inlet vane angles at tip and hub diameter, deg

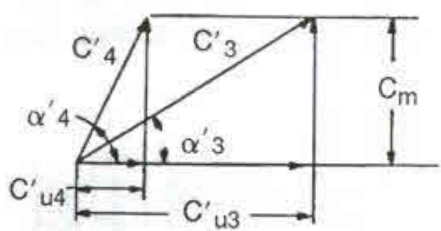

Impeller

Stator Vane

Elements and

Flow Velocity

Diagrams
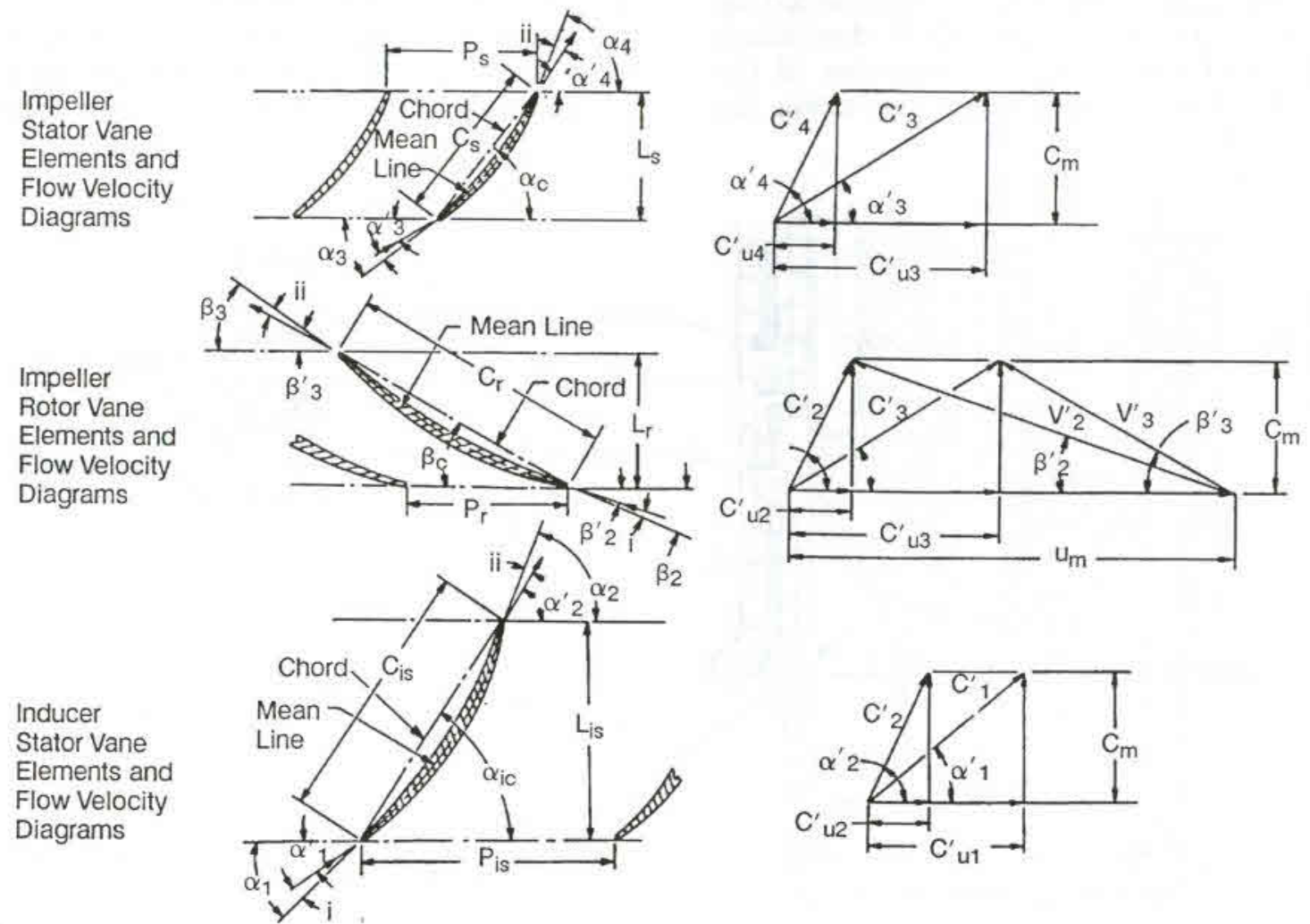

Fig. 6-49 Vane elements and flow-velocity diagrams of axial-flow pumps. 


\section{$\beta_{3 \mathrm{t}}, \beta_{3 \mathrm{~h}}=$ rotor-outlet vane angles at tip and hub diameter, deg \\ $\mathrm{u}_{\mathrm{t}}, \mathrm{u}_{\mathrm{h}}=$ rotor peripheral velocities at tip and hub diameter, $\mathrm{ft} / \mathrm{s}$

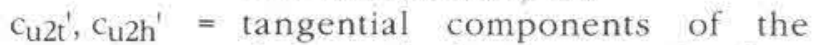 design absolute rotor inlet-flow velocities at tip and hub diameter, $\mathrm{ft} / \mathrm{s}$

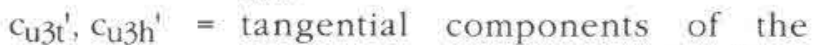 design absolute rotor outlet-flow velocities at tip and hub diameter, $\mathrm{ft} / \mathrm{s}$}

Some parameters in the equations above require further definition. The parameter $\varepsilon$ represents the ratio of effective flow area to the geometric area-a parameter introduced to express the blockage caused by the buildup of boundary layers, particularly at the hub and tip-shroud. Thus, this parameter will usually be smaller at stages further downstream. Estimation of this parameter is usually based on an empirical database, although solutions to the Navier-Stokes equations with an adequate turbulence model are also acceptable. The parameter $Q_{e}$ is used to account for any leakage introduced at the various points in the flow path. Calculation of leakage flows is generally done independently and successively iterated with the pump design. The incidence angle is chosen by the designer considering the efficiency, cavitation potential, and the required flow-range. The deviation angle "ii" must be calculated in terms of the real fluid effects or estimated using some empirically based estimating technique. One such technique that has been widely used, the "Carter's Rule" or "modified Carter's Rule", can be found in various references (e.g., Ref. 6-2 and 6-7). As can be seen from Eq. (6-47), calculation of the deviation angle to design a blade to get the correct discharge velocity is absolutely critical to obtaining the correct head.

\section{Function of the Stator}

The stator of an axial-flow pump converts a major portion of the tangential component of the absolute flow velocity leaving the rotor into static pressure by "straightening" the flow after it leaves the rotor. The stator-vane curvature is designed so that the fluid enters the vanes with minimum loss and leaves the stator with a reduced tangential component of the absolute flow velocity. The cross-sectional areas of the stator flow passages normal to the axial direction equals those of the rotor, thus maintaining the axial component of the absolute flow velocity. The dimensions $d_{t}$ and $d_{h}$ of the stator, (Fig. 6-48) can be treated as equal to the tip and hub diameters of the rotor. The solidity of the stator vanes generally increases from hub diameter $\left(\mathrm{d}_{\mathrm{h}}\right)$ to tip diameter $\left(d_{t}\right)$ because the vanes are supported structurally at the tip. The axial length $\left(\mathrm{L}_{\mathrm{S}}\right)$ of the stator vane at the mean effective diameter is usually made equal to that of the rotor $\left(\mathrm{L}_{\mathrm{r}}\right)$, but it is governed by the solidity and the required number of vanes.

Regarding Fig. 6-49, the velocity diagrams for the stator inlet and outlet are constructed with the assumption that the absolute flow velocities and angles at stator inlets and outlets equal the corresponding ones at the rotor outlets and inlets. This facilitates the design of multistage axial-flow pumps using uniform rotor and stator stages.

To deflect the fluid effectively, the inlet-vane angles $\left(\alpha_{3}\right)$ of the stator should be greater, by a few degrees, than the inlet absolute-flow angles $\left(\alpha_{3^{\prime}}\right)$; i.e., an angle of attack "i" should be allowed. Also, an angle "ii" should be allowed between outlet-vane angle $\left(\alpha_{4}\right)$ and outlet absolute-flow angle $\left(\alpha_{4}^{\prime}\right)$ for the deviation angle. The following correlations can be established for the vane and flow velocity diagrams of the stator (Fig. 6-49):

$$
\begin{aligned}
& \mathrm{P}_{\mathrm{s}}=\pi \mathrm{d}_{\mathrm{m}} / \mathrm{z}_{\mathrm{s}} \\
& \mathrm{S}_{\mathrm{s}}=\mathrm{C}_{\mathrm{s}} / \mathrm{P}_{\mathrm{S}} \\
& \alpha_{\mathrm{c}}=0.5\left(\alpha_{3}+\alpha_{4}\right) \\
& \mathrm{C}_{\mathrm{s}}=2 \mathrm{R}_{\mathrm{S}} \sin \left[0.5\left(\alpha_{4}-\alpha_{3}\right)\right] \\
&=\mathrm{L}_{\mathrm{s}} / \sin \alpha_{\mathrm{c}} \\
& \alpha_{3}=\alpha_{3^{\prime}}+\mathrm{i} \\
& \alpha_{4}=\alpha_{4^{\prime}}+\mathrm{ii} \\
& \mathrm{c}_{\mathrm{m}}=\mathrm{c}_{\mathrm{u} 3^{\prime}} \tan \alpha_{3^{\prime}}=\mathrm{c}_{\mathrm{u} 4^{\prime}} \tan \alpha_{4}^{\prime} \\
&=\mathrm{c}_{3^{\prime}} \sin \alpha_{3^{\prime}}=\mathrm{c}_{4}^{\prime} \sin \alpha_{4}^{\prime} \\
& \mathrm{d}_{\mathrm{m}} \tan \alpha_{3}=\mathrm{d}_{\mathrm{t}} \tan \alpha_{3 \mathrm{t}}=\mathrm{d}_{\mathrm{h}} \tan \alpha_{3 \mathrm{~h}} \\
&=\mathrm{d}_{\mathrm{x}} \tan \alpha_{3 \mathrm{x}} \\
& \mathrm{d}_{\mathrm{m}} \tan \alpha_{4}=\mathrm{d}_{\mathrm{t}} \tan \alpha_{4 \mathrm{t}}=\mathrm{d}_{\mathrm{h}} \tan \alpha_{4 \mathrm{~h}} \\
&=\mathrm{d}_{\mathrm{x}} \tan \alpha_{4 \mathrm{x}}
\end{aligned}
$$

where-

$$
\begin{aligned}
& \mathrm{P}_{\mathrm{S}}=\text { pitch or stator-vane spacing, in. } \\
& \mathrm{Z}_{\mathrm{S}}=\text { number of stator vanes } \\
& \mathrm{S}_{\mathrm{S}}=\text { stator-vane solidity } \\
& \mathrm{C}_{\mathrm{S}}=\text { stator-vane chord length, in. } \\
& \alpha_{c}=\text { stator-vane chord angle, deg } \\
& \alpha_{3}, \alpha_{4}=\text { vane angles at stator inlet and } \\
& \text { outlet, deg } \\
& \mathrm{R}_{\mathrm{S}}=\text { radius of the stator-vane curvature, } \\
& \mathrm{L}_{\mathrm{S}}=\text { axial length of the stator vane, in. } \\
& \mathrm{i}=\text { angle of attack, deg } \\
& \text { ii = angle allowed for circulatory flow } \\
& \text { at the outlet, deg } \\
& \alpha_{3}{ }^{\prime}, \alpha_{4}^{\prime}=\text { absolute-flow angles at stator inlet } \\
& \text { and outlet, deg } \\
& \mathrm{cm}=\text { axial component of the absolute- } \\
& \text { flow velocities, } \mathrm{ft} / \mathrm{s} \\
& \mathrm{c}_{3}{ }^{\prime}, \mathrm{c}_{4}^{\prime}=\text { design absolute-flow velocities of } \\
& \text { stator inlet and outlet, } \mathrm{ft} / \mathrm{s} \\
& \mathrm{c}_{\mathrm{u} 3^{\prime},}, \mathrm{c}_{\mathrm{u} 4}{ }^{\prime}=\text { tangential components of the } \\
& \text { design absolute velocities at stator } \\
& \text { inlet and outlet, } \mathrm{ft} / \mathrm{s} \\
& \alpha_{3 \mathrm{t}}, \alpha_{3 \mathrm{~h}}, \alpha_{3 \mathrm{x}}=\text { stator-inlet vane angles at tip, hub, } \\
& \text { and any intermediate diameter, } \\
& \text { deg } \\
& \alpha_{4 \mathrm{t}}, \alpha_{4 \mathrm{~h}}, \alpha_{4 \mathrm{x}}=\text { stator-outlet vane angles at tip, } \\
& \text { hub, and any intermediate di- } \\
& \text { ameter, deg }
\end{aligned}
$$




\section{Design of Impeller Rotors and Stators}

A number of design factors directly affect the performance characteristics of an axial-flow pump. Evaluation of test information, on the basis of specific speed per stage $\left(N_{S}\right) 1$, shows the following definitive correlations:

Impeller-bub ratio $\boldsymbol{r}_{\boldsymbol{d}}$. The ratio of impeller hub diameter $\mathrm{d}_{\mathrm{h}}$ to tip diameter $\mathrm{d}_{\mathrm{t}}$ (Fig. 6-48) has a direct bearing on the specific speed per stage $\left(\mathrm{N}_{\mathrm{s}}\right)_{1}$. Higher-specific-speed pumps have higher relative flows and therefore smaller hub ratios that result in greater free-flow area; but they also have lower head $(\mathrm{H} / \mathrm{Q})$ characteristics. However, a higher hub ratio tends to yield a higher head coefficient per stage $(\psi)_{1}$. Typical values of $r_{d}$ in rocket-engine hydrogen pumps range from 0.75 to 0.86 . Typical design values for $\left(N_{S}\right)_{1}$ and $(\psi)_{1}$ range from 3000 to 5000 and from 0.25 to 0.40 , respectively. A key factor that sets the head coefficient, the diffusion factor, will be discussed more fully below.

Vane solidities $\boldsymbol{S}_{\boldsymbol{n}} \boldsymbol{S}_{\boldsymbol{s}}$. The vane solidities or chord-spacing ratios of the rotor and statorimportant design parameters-are selected on the basis of previous experience. A higher pump specific speed is linked with lower solidity primarily because the required head is smaller and so gives the higher specific speed. Typical design values for vane solidities for the rotor and stator at the mean effective diameter $\left(\mathrm{d}_{\mathrm{m}}\right)$ range from 1 to 1.34 and 1.5 to 1.8 , respectively. Lower values are generally more efficient when the diffusion factor and head generated are lower.

Number of vanes $z_{r}, z_{s}$. A lower pump specific speed usually results in a larger number of vanes. Design values of $\mathrm{z}_{\mathrm{r}}$ range from 14 to 20 . Design values of $z_{S}$ are typically higher, up to a factor of approximately 2 . To minimize the potential for dynamic interaction, the number of stator vanes should have no common factor with the number of rotor blades.

Vane curvature and setting. Experiments indicate that the head developed by the rotor is essentially determined by vane curvature, i.e., $\beta_{3}-\beta_{2}$. Changes in vane settings (i.e., outlet-vane angle $\beta_{3}$ and inlet-vane angle $\beta_{2}$ ) by the same amount will not affect head rise and efficiency significantly.

The design procedure for the impeller rotors and stators of a multi-stage axial-flow pump is essentially the same as that for a single-stage centrifugal pump, except for the determination of the number of pump stages. Design parameters and coefficients established experimentally using prior successful designs should be utilized to the fullest. Special development tests will still be required to verify the characteristics of a new design. The design procedure should include the following steps:

1) To meet a given set of engine system requirements such as rated design pump-developed head $\mathrm{H}$, flow rate $\mathrm{Q}$, and rated pump (NPSH)c, the pump rotating speed $\mathrm{N}$ is determined first through selection of a suitable inducer of a given suction specific speed $\left(\mathrm{N}_{\mathrm{SS}}\right)_{\text {ind }}$.
2) With $\mathrm{N}$ established, selection of impeller rotor and stator of a given specific speed per stage $\left(\mathrm{N}_{\mathrm{S}}\right)_{1}$, combined with a determination of the number of pump stages, can proceed with the aid of the following correlations:

$$
\begin{gathered}
\left(\mathrm{N}_{\mathrm{S}}\right)_{1}=\mathrm{N}(\mathrm{Q})^{0.5} /(\Delta \mathrm{H})_{1} 0.75 \\
\Delta \mathrm{H}=\Delta \mathrm{H}_{\text {ind }}-\mathrm{H}_{\mathrm{ee}}+\mathrm{n}(\Delta \mathrm{H})_{1}
\end{gathered}
$$

where-

$$
\begin{aligned}
\left(\mathrm{N}_{\mathrm{S}}\right)_{1}= & \text { specific speed per axial-flow-pump } \\
& \text { stage } \\
\mathrm{N}= & \text { rated design pump rotating speed, } \\
& \text { rpm } \\
\mathrm{Q}= & \text { rated design pump flow rate, gpm } \\
(\Delta)_{1}= & \text { rated design pump-developed head } \\
& \text { per axial-flow pump stage, } \mathrm{ft} \\
\Delta \mathrm{H}= & \text { rated design pump-overall-developed } \\
& \text { head, } \mathrm{ft} \\
\Delta \mathrm{H}_{\mathrm{ind}}= & \text { inducer rated head rise, } \mathrm{ft} \\
\mathrm{H}_{\mathrm{ee}}= & \text { hydraulic-head loss at the inducer } \\
& \text { stator, } \mathrm{ft} \\
\mathrm{n}= & \text { number of axial-flow pump stages }
\end{aligned}
$$

3) For the specific speed per stage $\left(N_{S}\right)_{1}$ thus obtained, various design factors and coefficients such as impeller hub ratio $r_{d}$, vane solidities $S_{r}$ and $S_{S}$, number of vanes $z_{r}$, and $z_{S}$, head coefficient per stage $(\psi)_{1}$, etc., will be selected based on past designs with comparable $\left(\mathrm{N}_{\mathrm{s}}\right)_{1}$ values.

4) The required impeller rotor and stator diameters, velocity diagrams, and vane profiles can now be derived from Eq. (6-37) through (6-63).

Impeller rotor and stator vanes are usually machined from forgings of aluminum alloys or nickel-base alloys such as K-Monel. In view of the relatively low head produced by an individual axialflow-impeller stage, reduction of skin-friction and flow-turbulence losses prove more important than with centrifugal pumps. High efficiency demands a high degree of vane streamlining and polishing.

The axial distance $d_{a}$ between impeller rotor vanes and stator vanes (Fig. 6-49) has some bearing on performance. Typical design values of $d_{a}$ range from 0.02 to $0.05 d_{t}$, where $d_{t}$ equals the impeller-tip diameter. This spacing is also expressed as a function of the chord of the upstream blade. In general, the spacing should not be less than $10 \%$ of this chord to avoid large dynamic stresses because of the interaction of the rotor-stator blades. As improved CFD codes are being developed, the dynamic stresses can be calculated to give guidance to the design. Design values for tip clearance $\mathrm{c}$ for both the rotor and stator (Fig. 6-46) should be no more than approximately $2 \%$ of the blade or vane height. Smaller values can be used depending on the manufacturing tolerances and the dynamic behavior of the pump that controls the rotor's radial motion.

The vane thickness along the mean line will mainly be determined by structural considerations because experiments indicate that there is very little effect on performance from variation of vane thickness as long as the leading and trailing edges 
are kept at a small radius and the thickness is developed along the meanline in an efficient manner. The problems with calculating stresses for pump rotor vanes are similar to those with turbine blades. The methods given in section 6.6 for turbines can also be applied here. Vanes experience centrifugal, bending (due to lift and drag loading), and vibrational stresses.

\section{Diffusion and Retardation Factors}

In designing the axial pump, it is desirable to increase the head per stage to reduce the total number of stages. However, the head per stage is limited by the ability of the blading to turn the flow without inducing a separation that causes pump stall, an undesirable operating condition. Usually the peak efficiency of the design will be maximum as the head per stage is increased, but design margins must be maintained to avoid stall. To assess the stall potential and the losses in an axial stage, a "parameterdiffusion" factor has been developed; it has been shown to be a reliable indicator. Using the vector diagrams of Fig. 6-49, the diffusion factor for the rotor can be expressed as follows:

$$
\mathrm{DF}_{\mathrm{r}}=1.0-\left(\mathrm{v}_{3}^{\prime} / \mathrm{v}_{2}^{\prime}\right)+\left(\mathrm{cu}_{3}^{\prime}-\mathrm{cu}_{2}^{\prime}\right) /\left(2 \mathrm{~S}_{\mathrm{r}^{\prime}} \mathrm{v}^{\prime}\right)
$$

Similarly, for the stator:

$$
\mathrm{DF}_{\mathrm{S}}=1.0-\left(\mathrm{c}_{4}^{\prime} / \mathrm{c}_{3}^{\prime}\right)+\left(\mathrm{cu}_{4}^{\prime}-\mathrm{cu}_{3}^{\prime}\right) /\left(2 \mathrm{~S}_{\mathrm{S}^{\prime}} \mathrm{c}^{\prime}\right)
$$

Good efficiencies at reasonable stall margins have been achieved with the diffusion factors in the range of 0.45 to 0.55 . Diffusion factors above this range have been successfully used, but require a more sophisticated analysis to assure that stall will be avoided. Designs have been achieved that push the stall up to a diffusion factor of 0.75 ; obviously, the higher the diffusion factor, the smaller will be the unstalled flow range.

Also used to estimate the stall condition has been a "retardation" factor. Using again the vectors of Fig. 6-49, the retardation factor for the rotor is given by $\left(v_{3}^{\prime} / v_{2}^{\prime}\right)$ and for the stator by $\left(c_{4}^{\prime} / c_{3}^{\prime}\right)$. The designer should assume that stall will occur whenever the retardation factor reaches 0.5 . Successfull designs have been achieved with this factor as low as 0.63 .

\section{Design of Casings for Axial Pumps}

As shown in Fig. 6-6 and 6-50, the casing of an axialflow pump consists of a cylindrical section housing the inducer and impeller stages. It also includes a volute section with radial guide vanes situated behind the last rotor stage of the impeller. In addition to converting the tangential-flow-velocity component into pressure, the volute section also serves to reduce the axial-velocity component by gradually increasing the flow area toward the volute discharge.

The radial guide vanes of the volute section are designed so that the fluid enters them with minimum losses and leaves them in a radial plane, analogous to a centrifugal pump (Fig. 6-43). The number of radial guide vanes will be chosen to achieve the desired turning with a reasonable diffusion factor in the space provided. They should have no common factor with the number of impeller rotor blades.

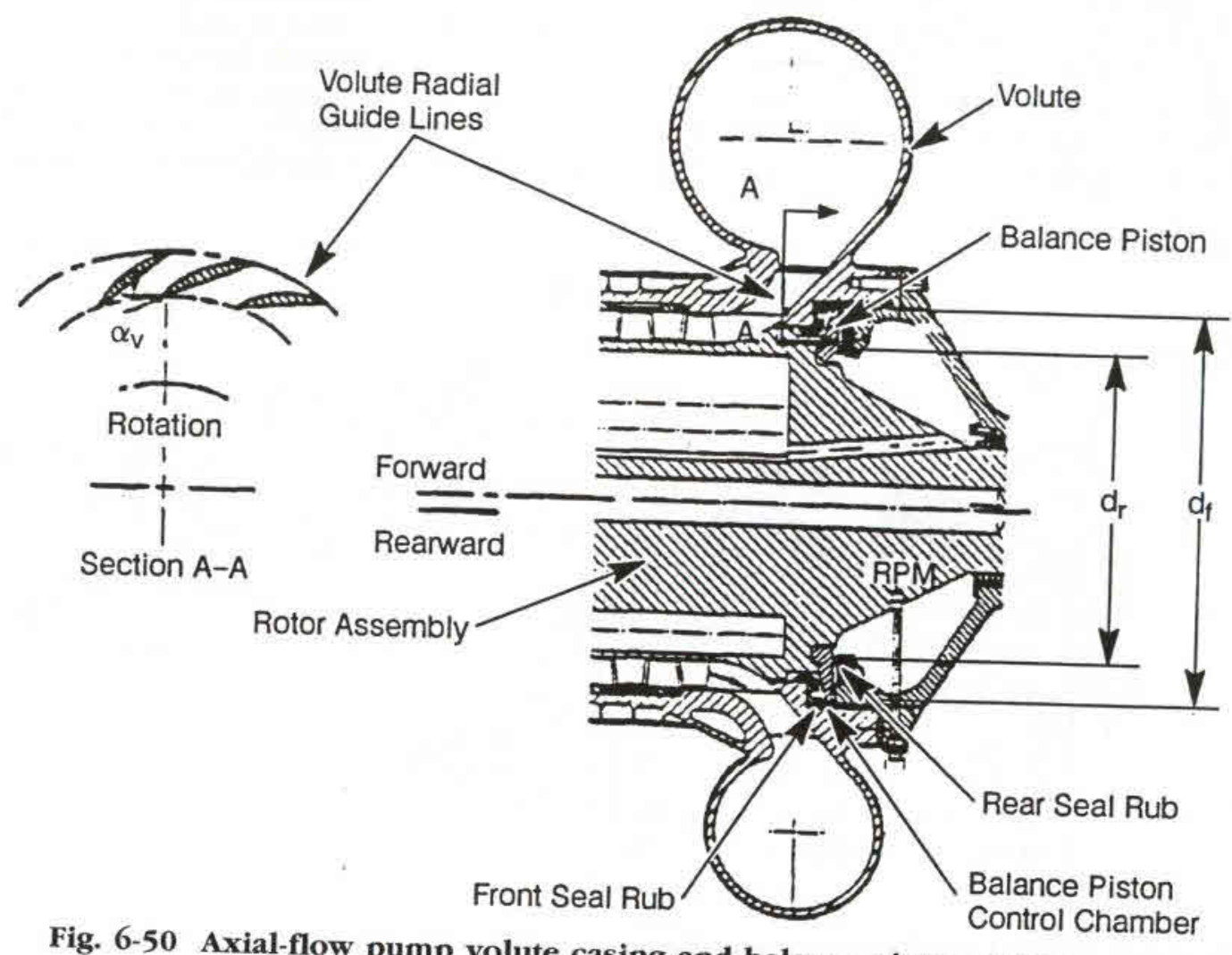


Vane angle $\left(\mathrm{a}_{\mathrm{v}}\right)$ can be determined by constructing the flow velocity diagram for that section.

The design calculations for the volute are the same as for a centrifugal-pump volute. This includes the area distribution, cross-sectional shape, and the volute tongue angle.

\section{Balancing the Axial Thrust of MultiStage Axial Pumps}

Balancing of the combined axial thrust of a multistage axial-flow pump is an important function because of the high pressures involved. Special balancing devices, such as automatic balance pistons, are frequently used. Secured to the rotor assembly, as shown in Fig. 6-50, the balance piston operates on the same principle as the balance piston of a centrifugal pump, consisting of a disk with small clearances and a pair of seal rubs located on either side of the disk. The balance piston has a forward movement (toward the inlet) of the rotor assembly. That reduces the clearance at the front-seal rub, simultaneously increasing clearances at the rear-seal rub. As a result, the pressure drops in the control chamber between front and rear seal rubs. This effect counteracts the forward hydraulic axial thrust of the rotor assembly. Similarly, a pressure increase in the control chambers counteracts a rearward movement (reverse thrust) of the rotor assembly. The volume to the rear of the control chamber communicates with the low-pressure region of the pump inlet through cavities in the rotor.

With the smaller diameter of the axial-flow pump, the available area for axial thrust control will usually be smaller, and thus requires higher pressures to achieve similar thrust ranges. The higher pressures also lead to higher leakage rates, which can penalize the pump efficiency. Thus, the final performance comparison of axial- vs. centrifugalpump design must include evaluation of the effects of the balance-piston design.

\subsection{TURBINE DESIGN}

Gas-generator-cycle rocket engine applications will favor impulse turbines for their simplicity, high power-to-weight ratio, and low axial thrust (Ref, 6-8). Figure 6-51 shows the general arrangement of a typical single-stage, two-rotor, velocity-compounded impulse turbine. Gas-generator-cycle turbines are characterized by relatively high pressure ratios (e.g., as high as 20 for a single stage) with supersonic velocities and low flowrates. Turbine flowrate is minimized in a gas-generator cycle to maximize engine specific impulse because the turbine flowrate bypasses the engine thrust chamber and produces a relatively low thrust. In some cases the turbine flowrate may be low enough to require partial-admission turbine nozzles (nozzles flowing around only part of their circumference) for higher performance.

Staged-combustion-cycle and series-expandercycle rocket engine applications will use low-reaction turbine stages to maximize turbine performance. Figure 6-52 shows the two-stage reaction turbine in the SSME HPFTP. Staged-combustion and expandercycle turbines are characterized by relatively low pressure ratios (e.g., less than 2 for two stages) with subsonic velocities and high flowrates. Turbine pressure ratio will be kept as low as possible to minimize the pump-discharge pressure required to achieve a given main-chamber pressure and pump power requirements. The low-reaction staging minimizes the maximum velocities in the stage, resulting in maximum efficiency. In high-chamber-pressure rocket engines, the low pressure ratios still result in high turbine-rotor pressure drops and high axial thrust of the turbine.

A turbine stage consists of an expanding stationary-vane row (nozzle) followed by a single row or multiple rows of rotating blades. Low expansions may occur in the rotating-blade rows and redirecting stator. A second expanding nozzle begins another stage. The total available energy is divided between the stages based on the desired power split.

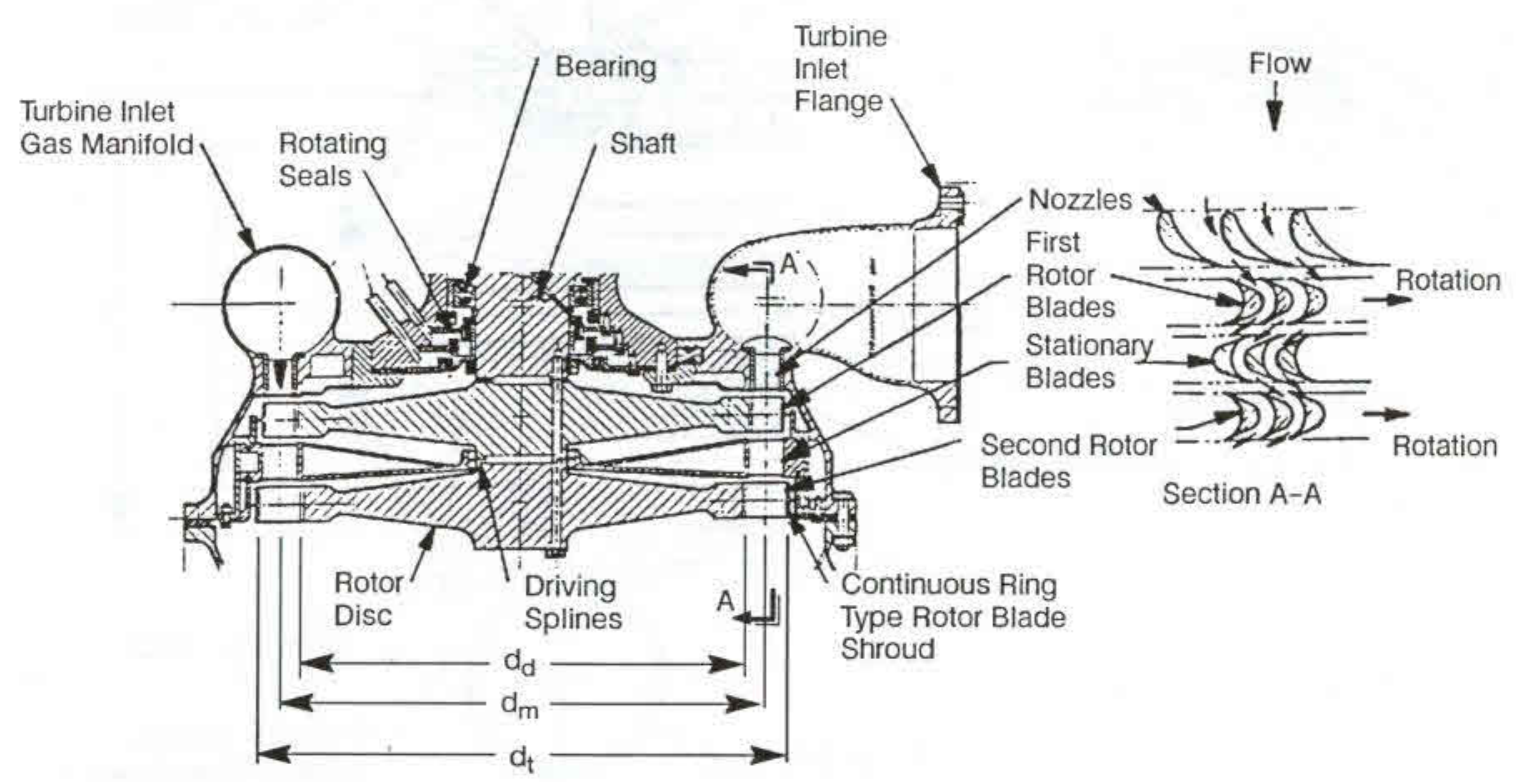

Fig. 6-51 Typical single-stage, two-rotor, velocity-compounded impulse turbine. 


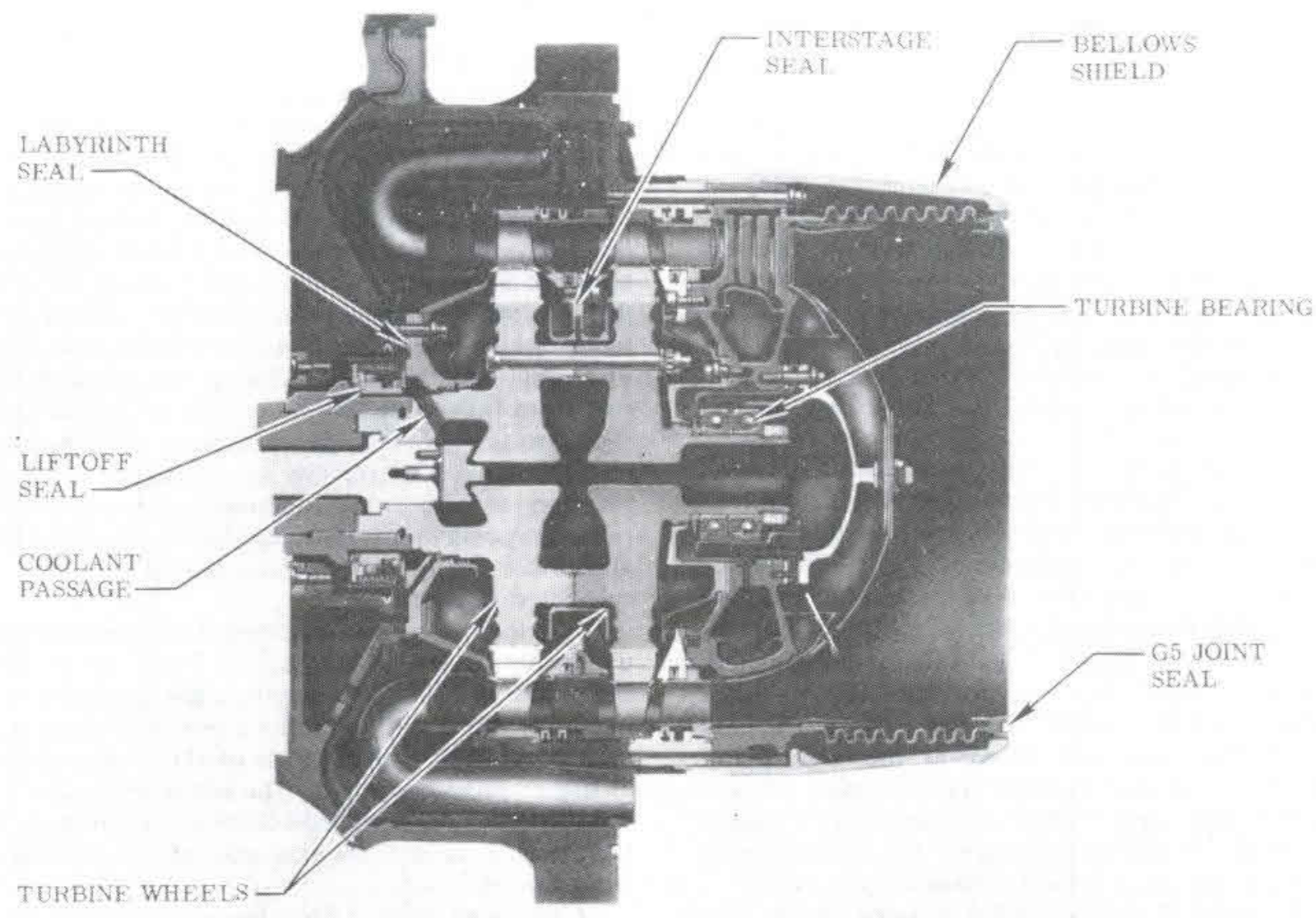

Fig. 6-52 Two-stage reaction turbine for SSME HPFTP.

\section{General Design Procedure}

An integrated turbine aerothermodynamic design will have overall requirements specified by a rocketengine balance calculation. The following steps (Fig. 6-53) are essential in the design of a rocket-engine turbine.

Selection of turbine type. The first item of importance is the selection of the proper type. A single-stage rotor turbine (Fig. 6-10) will be used if the required turbine power is low, if the efficiency of the turbine has less effect on overall engine system performance, or if the pressure ratio is high and partial admission is required for high performance. When the available energy of the turbine workingfluid, and therefore the gas spouting velocity $\mathrm{Co}$ is relatively low, a higher turbine velocity ratio $\mathrm{U} / \mathrm{Co}$ may be achieved with a moderate turbine rotorblade speed U. As shown in Fig. 6-27, this suggests the use of a relatively simple single-stage, singlerotor impulse turbine. This type was selected for the A-2 Stage oxidizer turbopump, at the same time taking advantage of its overall simplicity.

In most direct-drive turbopump configurations (such as the A-1 Stage engine turbopump, Fig. 6-79), where the turbine rotating speed $\mathrm{N}$ and consequently turbine velocity ratio $\mathrm{U} / \mathrm{Co}$ is lower than peak efficiency, a single-stage, two-rotor, velocity-compounded impulse turbine (Fig. 6-11 and 6-51) will be

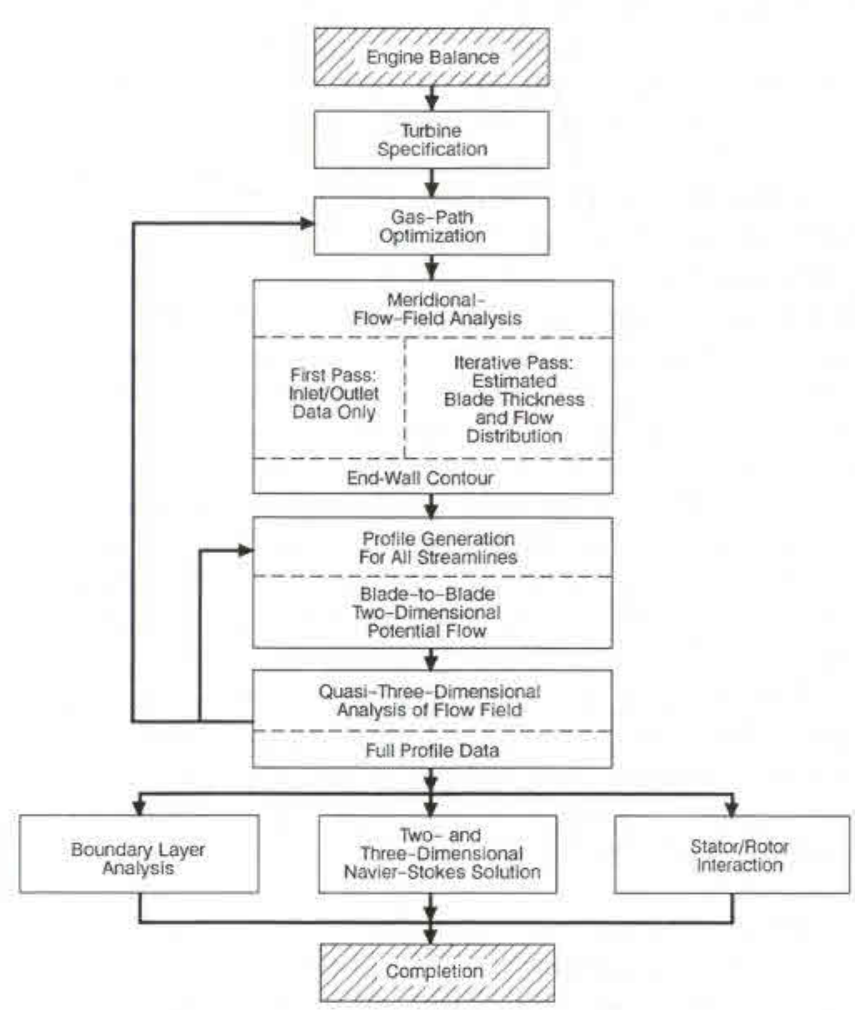

Fig. 6-53 Typical steps in the turbine design process. 
selected for best results. Figure 6-27 indicates that the optimum efficiency of a velocity-compounded turbine can be achieved at a relatively low U/Co value.

The turbine can operate at a much higher rotating speed (over 25,000 rpm) if a reduction gear train couples the pump and the turbine (Fig. 6-16). A higher value of $\mathrm{U} / \mathrm{Co}$ can be achieved with reasonable turbine rotor size. Then a higher-performance, two-stage, two-rotor, pressure-compounded impulse turbine (Fig. 6-12) may be used.

Low-reaction staging (about 25\% reaction) enables low turbine pressure ratios to maximize efficiency. Stage velocity ratio U/Co and efficiency can be increased and blade loading reduced by using two low-reaction stages. The power split between stages typically has been about equal.

Size of turbine. After selecting the type of turbine, the designer determines the turbine-rotor size. Once the characteristics of the turbine working fluid, the turbine pressure ratio $\mathrm{R}_{\mathrm{t}}$, and the pump or turbine rotational speed $\mathrm{N}$ have been established, choosing a larger diameter for the turbine rotor tends to result in a higher velocity ratio $\mathrm{U} / \mathrm{Co}$, and thereby higher efficiency. However, the larger-diameter turbine rotor also spells higher assembly weight, larger envelope, and higher working stresses. Final selection of the turbine-rotor size, and consequently the $\mathrm{U} / \mathrm{Co}$ ratio, often entails a design compromise,

Determine flowrate and pressure ratio. The required power output from the turbine must be equal to the net input to the propellant pumps, plus the mechanical losses in the bearings, seals, gears (if any), plus the net power required to drive any auxiliary drives. The required flowrate of the turbine working fluid can be calculated using Eq. (6-15) after the required power, available energy of the working fluid [Eq. (6-13)], and the overall turbine efficiency (from Fig. 6-27 in the initial phases of design) have been estimated,

Dimensions of blade rows. The initial dimensions of the stationary nozzles and of the rotating blades can be calculated using a preliminarydesign pitch-line, gas-path program. The pitch-line analysis includes the selected staging, pressure distribution, and flow angles as well as representative losses due to friction and turning for each blade row, tip-clearance losses, partial-admission losses, rotordisk windage losses, and shaft- and interstage-seal leakage losses. The blade-row outlet flow area can be calculated using continuity for the outlet conditions. Stage power, efficiency, and reaction are calculated from the Euler equation, pressure distribution, and the losses. The interactive program allows the designer to alter the various design parameters to achieve the optimum configuration within the design constraints. Computer graphics can display the resulting velocity-vector diagrams and blade-path geometry.

When blades are relatively short (height-toradius ratio less than 0.10 ) and when turbine efficiency does not have a strong effect on engine cycle performance, constant-section blading can be used to lower cost and gain good performance. The radial-pressure variation is determined for constant- flow-angle blading to determine axial thrust. Constant-section blading usually employs relativelyshort, high-pressure-ratio supersonic blades.

For longer turbine blades (usually in subsonic turbine stages), a free-vortex radial distribution with twisted blades may be selected. Higher efficiency results from twisted blades due to reduced-incidence losses and more uniform flow from hub to tip. The ideal characteristics of a free-vortex design are constant axial velocity and rotor work from hub to tip. (Actual operation with efficiency gradients hub to tip cause variations in both velocity and work.) The tangential absolute velocity out of each blade row from hub to tip can be calculated assuming that the product of the mean radius and the mean absolute tangential velocity is a constant. The rotor reaction at the mean diameter should be set to provide positive reaction at the hub. Inlet-blade angles are set to minimize incidence losses for the calculated flow-angles.

Efficiency can be increased to the maximum by using a forced-vortex radial work distribution. Increased work is performed in the lower-loss midheight of the blade and reduced work is done in the higher-loss hub and tip ends of the blade, resulting in higher stage efficiency. The reduced tip work also reduces the unshrouded-blade tip clearance loss. Figure 6-54 shows an example of a forced-vortex design rotor blade.

CFD analyses. The design and analyses of forced-vortex-stage 3D blading requires extensive computer graphics and computational fluiddynamics (CFD). Computer-aided engineering (CAE) graphics must define, stack, and display the three-dimensional

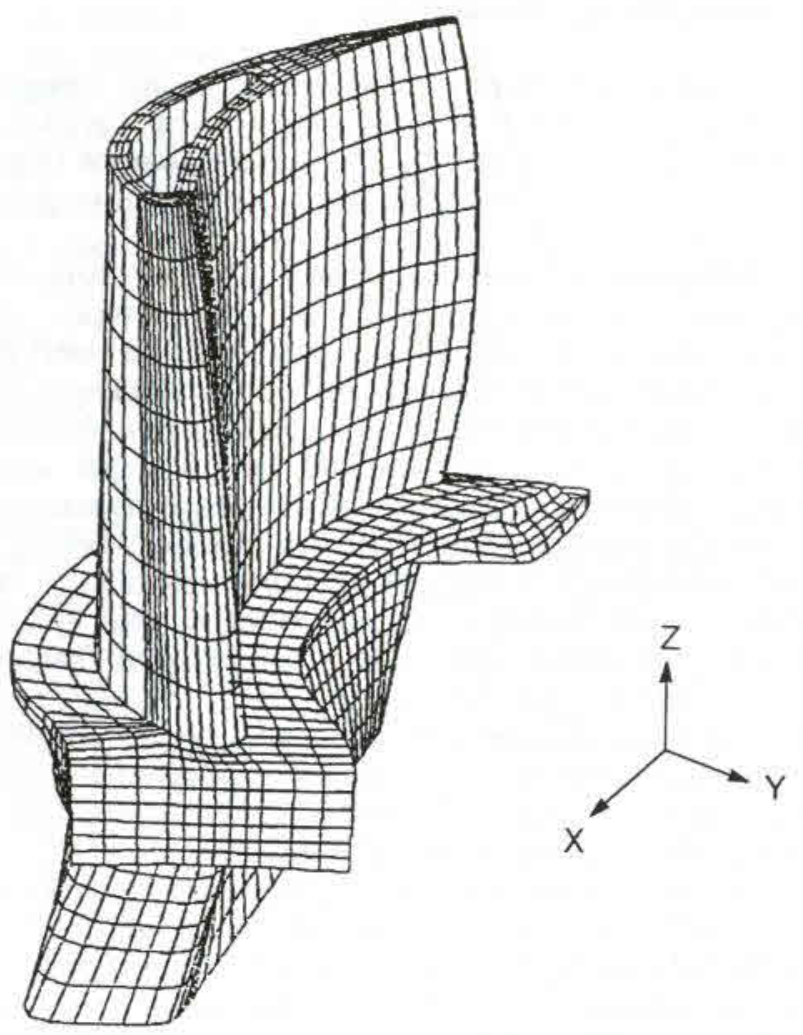

Fig. 6-54 Example of a forced-vortex-design rotor blade. 
blade shapes and establish the input data to the three-dimensional analysis programs. The detail design analysis that establishes the flowpath elevation and blade-profile dimensions may be done using an inviscid quasi-3D or full-3D analysis. The vane and blade surface distributions of velocity, Mach number, static pressure, and temperature are calculated from hub to tip for each blade row. The surface boundary layers are calculated assuming turbulent flow for the high-Reynolds-number combustor products of the rocket engine. The blade shapes are modified until the surface distributions and boundary layers have acceptable diffusion levels and minimum loss and separation potential.

Blade-profile designs finalized, the viscous-flow solvers may be executed to verify the viscous effects. When the stage design is completed, the nozzle/ rotor interaction should be analyzed to establish the blade integrity under dynamic loading.

\section{Design of Turbine Nozzles}

Turbine nozzle designs are classified as convergentdivergent for high-pressure-ratio, supersonic, singlestage turbines, convergent for subsonic and transonic stages, and partial admission when the rotorblade height would be too small for full admission.

Supersonic convergent-divergent nozzles. The nozzles of most gas-generator-cycle rocketengine turbines are basically similar to those of rocket thrust chambers. They are of the conventional converging-diverging De Laval type with supersonic outlet velocities. In the main, the nozzles of an impulse-type turbine efficiently convert the major portion of available energy of the working fluid into kinetic energy or high gas-spouting velocity. The gas flow processes in the thrustchamber nozzles are directly applicable to turbine nozzles. However, the gas flow in an actual nozzle deviates from ideal conditions because of fluid viscosity, friction, boundary-layer effects, etc. In addition, the energy consumed by friction forces and flow turbulence will cause an increase in the static temperature of the gases flowing through a nozzle, above that of an isentropic process. (This effect is known as "reheat.") As a result of the above effects, the actual gas-spouting velocity at the turbine nozzle exit tends to be less than the ideal velocity calculated for isentropic expansion (from stagnation state at the nozzle inlet to the static pressure at the rotor-blade inlet). Furthermore, the effective flow area of a nozzle will usually be less than the actual area because of circulatory flow and boundary-layer effects. The following correlations have been established for the design calculations of turbine nozzles:

Nozzle velocity coefficient $k_{n}$ :

$$
\mathrm{k}_{\mathrm{n}}=\mathrm{C}_{1} / \mathrm{Co}
$$

where $C_{1}=$ actual gas spouting velocity at the nozzle exit, and $\mathrm{Co}$ is the ideal gas velocity calculated for isentropic expansion from the stagnation state at the nozzle inlet to static pressure at the rotor blade inlet.
Nozzle efficiency $\eta_{n}$ :

$$
\eta_{\mathrm{n}}=\mathrm{k}_{\mathrm{n}}^{2}
$$

Nozzle throat area coefficient $e_{n t}$

$$
\begin{aligned}
\varepsilon_{\mathrm{nt}}= & (\text { effective area of the nozzle throat }) / \\
& \text { (actual area) }
\end{aligned}
$$

Actual gas-spouting velocity at the nozzle exit, $\mathrm{ft} / \mathrm{s}$ :

$$
\begin{aligned}
\mathrm{C}_{1} & =\mathrm{k}_{\mathrm{n}} \mathrm{C}_{\mathrm{o}}=\mathrm{k}_{\mathrm{n}} \sqrt{2 \mathrm{gJC}_{\mathrm{p}} \mathrm{T}_{\mathrm{o}}\left[1-\left(\frac{\mathrm{p}_{1}}{\mathrm{p}_{\mathrm{o}}}\right)^{\frac{\gamma-1}{\gamma}}\right]} \\
& =\mathrm{k}_{\mathrm{n}} \sqrt{2 \mathrm{~g} J \Delta \mathrm{H}_{\mathrm{o}-1^{\prime \prime}}}
\end{aligned}
$$

Amount of nozzle rebeat:

$$
\mathrm{q}_{\mathrm{nr}}=\frac{\left(1-\mathrm{k}_{\mathrm{n}}^{2}\right) \mathrm{C}_{1}^{2}}{\mathrm{k}_{\mathrm{n}} 2 \mathrm{gJ}}=\frac{\left(-\eta_{\mathrm{n}}\right) \mathrm{C}_{1}^{2}}{\eta_{\mathrm{n}} 2 \mathrm{gJ}}
$$

Required total nozzle throat area, in 2 :

$$
\mathrm{A}_{\mathrm{nt}}=\frac{\dot{\mathrm{w}}_{\mathrm{t}}}{\epsilon_{\mathrm{nt}} \mathrm{p}_{\mathrm{o}} \sqrt{\frac{\mathrm{g} \gamma\left[\frac{2}{\gamma+1}\right]^{\frac{\gamma+1}{\gamma-1}}}{\mathrm{RT}_{\mathrm{o}}}}}
$$

where-

$\mathrm{C}_{\mathrm{p}}=$ turbine-gas (working-fluid) specific heat at constant pressure, $\mathrm{Btu} / \mathrm{lb}-{ }^{-\mathrm{R}}$

$\gamma=$ turbine-gas specific heat ratio

$\mathrm{R}=$ turbine-gas constant (1544/molecular weight), $\mathrm{ft} /{ }^{\circ} \mathrm{R}$

$\mathrm{T}_{\mathrm{O}}=$ turbine-gas total (stagnation) temperature at the nozzle inlet, $R$

$\mathrm{W}_{\mathrm{t}}=$ turbine-gas mass flow rate, $\mathrm{lb} / \mathrm{s}$

$\mathrm{p}_{\mathrm{O}}=$ turbine-gas total pressure at the nozzle inlet, psia

$\mathrm{p}_{1}=$ turbine-gas static pressure at the rotorblade inlet, psia

$\Delta \mathrm{H}_{\mathrm{O}-1 "}=$ isentropic enthalpy drop of the gases flowing through the nozzles, due to expansion, Btu/lb

The performance of a turbine nozzle, as expressed by its efficiency or velocity coefficient, will be affected by a number of design factors, such as; exit velocity of the gas flow, properties of the turbine gases, angles and curvatures at nozzle inlet and exit, radial height and width at the throat, and pitch (spacing) and number of nozzles. Design values for the efficiency and velocity coefficients of a given turbine nozzle may be determined experimentally or estimated from past designs. Design values of nozzle efficiency $\eta_{\mathrm{n}}$ range from 0.80 to 0.96 . Design values of nozzle velocity coefficient $\mathrm{k}_{\mathrm{n}}$ vary from 0.89 to 0.98 . The nozzle-throat area coefficient $\left(\varepsilon_{\mathrm{nt}}\right)$ generally will increase with nozzle radial height, design values ranging from 0.95 to 0.99 .

Rocket-turbine nozzles have rectangular cross section (Fig. 6-55). Nozzle vanes are closely spaced 


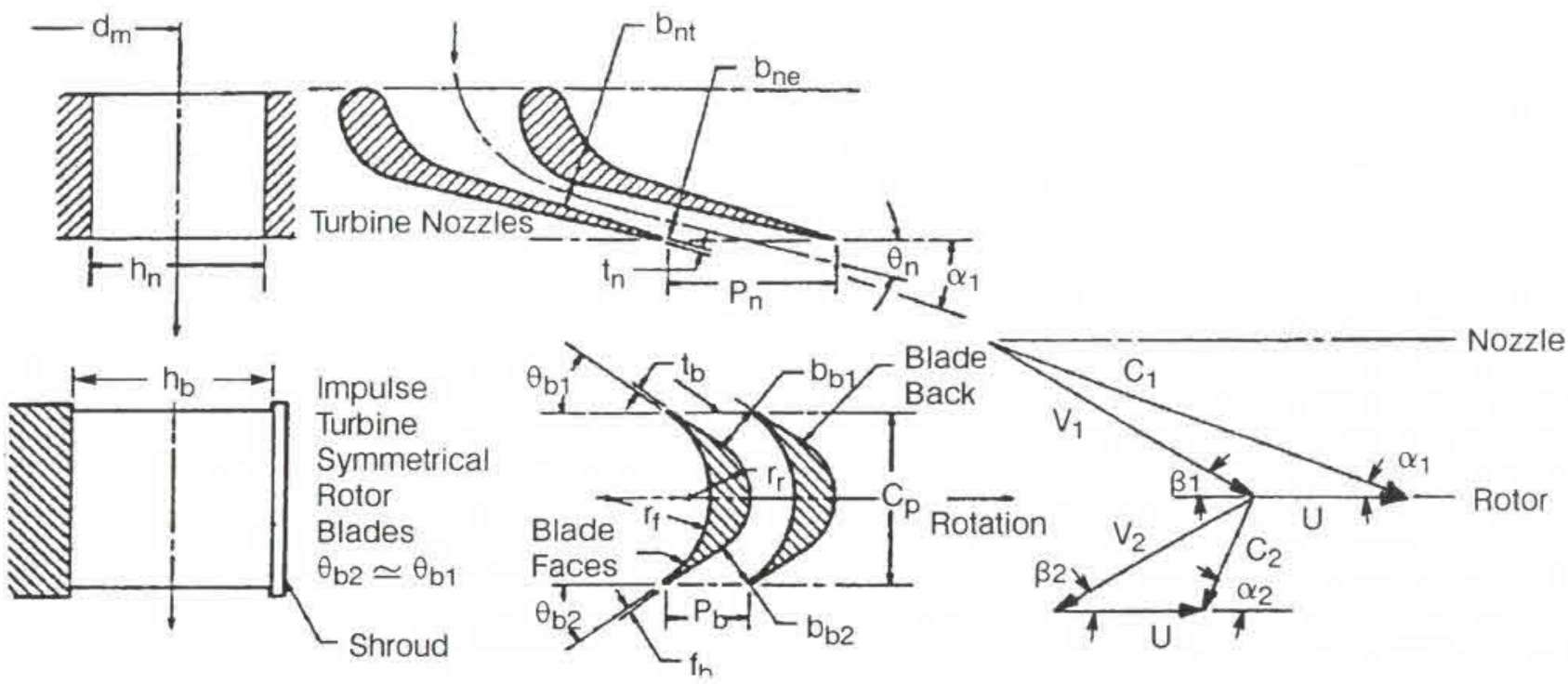

Velocity Diagrams of a Typical Single-Stage

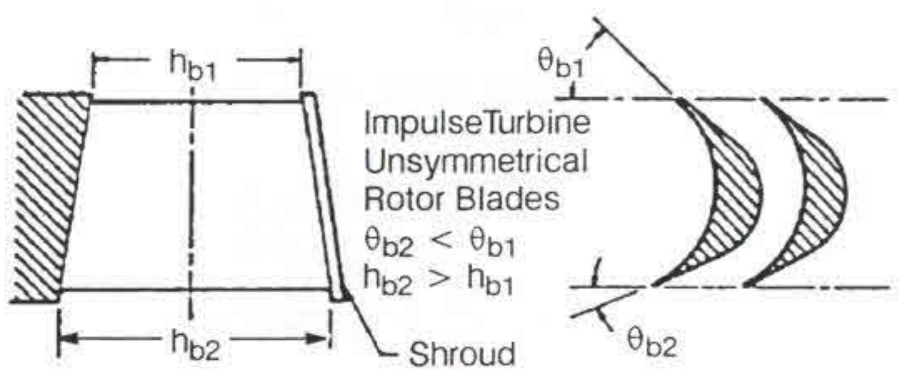
Impulse Turbine

Fig. 6-55 Nozzles, rotor blades, and velocity diagrams of a typical single-stage impulse turbine.

on a circular arc extending over a part of (partial admission) or all of (full admission) the circumference. Most high-power turbines use full admission for better performance.

While the gases are passing through a nozzle and expanding, the direction of flow is changing from an approximately axial direction to one forming the angle $\alpha_{1}$ (Fig. 6-55) with the plane of rotation, at the nozzle exit. Therefore, the turning angle is $90-\alpha_{1} \mathrm{deg}$. The angle $\left(\theta_{n}\right)$ of the nozzle centerline at the exit usually represents a design compromise. Theoretically, a smaller nozzle-exit-angle yields better efficiency, since the rotor blading work is larger and the absolute flow velocity at the rotor-blade exit is smaller. However, a smaller nozzle-exit-angle means a larger angle of flow deflection within the nozzle, which causes higher friction losses. Design values of $\theta_{\mathrm{n}}$ range from 15 to $30 \mathrm{deg}$. The actual effective discharge angle $\alpha_{1}$ of the gas jet leaving the nozzle tends to be greater than $\theta_{\mathrm{n}}$ because of the unsymmetrical nozzle shape at the exit.

A sufficiently large nozzle passage aspect ratio $h_{n t} / b_{n t}$ is desirable for better nozzle efficiency. For a given nozzle height, an increase in aspect ratio can be secured by decreasing the nozzle pitch $P_{n}$. However, a small pitch and consequently a large number of nozzles $\left(\mathrm{z}_{\mathrm{n}}\right)$, with attendant increase in wall surface, tends to increase friction losses.
Determination of nozzle pitch also requires a design compromise. The following correlations have been established for calculation of nozzle-flow areas:

Total nozzle throat area, in. 2 :

$$
A_{n t}=z_{n t} b_{n t} h_{n t}
$$

Total nozzle exit area, in.2:

$$
\begin{aligned}
A_{n e} & =144 \dot{w}_{t} /\left(\rho_{1} C_{1} \varepsilon_{n e}\right)=z_{n} b_{n e} h_{n e} \\
& =z_{n} h_{n e}\left(P_{n} \sin \theta_{n}-t_{n}\right)
\end{aligned}
$$

Pitch or nozzle spacing:

$$
\mathrm{P}_{\mathrm{n}}=\pi \mathrm{d}_{\mathrm{m}} / \mathrm{z}_{\mathrm{n}}
$$

where-

$\dot{\mathrm{w}}_{\mathrm{t}}=$ turbine-gas mass flowrate, $\mathrm{lb} / \mathrm{s}$

$\rho_{1}=$ density of the gases at nozzle exit, $\mathrm{lb} / \mathrm{ft}^{3}$

$\mathrm{C}_{1}=$ gas-spouting velocity at nozzle exit, $\mathrm{ft} / \mathrm{s}$

$\varepsilon_{\text {ne }}=$ nozzle-exit-area coefficient

$\mathrm{h}_{\mathrm{nt}}=$ radial height at nozzle throat, in

$\mathrm{h}_{\mathrm{ne}}=$ radial height at nozzle exit, in.

$\mathrm{b}_{\mathrm{nt}}=$ width normal to flow at nozzle throat, in.

$b_{\text {ne }}=$ width normal to flow at nozzle exit, in. 


$$
\begin{aligned}
z_{n}= & \text { number of nozzles } \\
\theta_{n}= & \text { angle between nozzle-exit centerline and } \\
& \text { plane of rotation, deg } \\
t_{n}= & \text { thickness of nozzle partition at exit, in. } \\
d_{m}= & \text { mean diameter of nozzles and rotor } \\
& \text { blades, in. }
\end{aligned}
$$

Subsonic convergent nozzles. Stagedcombustion and series expander-engine-cycle-drive turbines use subsonic convergent nozzles. The nozzle turns and accelerates the flow to the desired outlet Mach number from the inlet conditions. The nozzle passage converges with the minimum flow area at the outlet of the control channel (the throat). Additional expansion and turning may occur downstream of the throat. Nozzles with relatively short vanes can have constant section from hub to tip. The minimum passage width may be set by manufacturing constraints that would also affect the number of nozzle vanes. The height-to-throat opening ratio has typically been between 3.5 to 8 . The trailing-edge thickness typically varies between 5 to $15 \%$ of the throat opening. The fillet radius at the endwalls usually will be less than $30 \%$ of the throat opening. The number of subsonic nozzles used in rocket turbines has varied from 25 to 61 . Nozzle efficiency values range to 0.97 . Radial variations from hub to tip become significant for longer nozzles. The nozzle-outlet flow angle and area distributions are set to achieve the desired work distribution.

Laid out using computer graphics and CFD analyses, nozzle profiles are designed to achieve the required acceleration and turning for the desired stage distributions. Numerous profiles are defined from hub to tip and analyzed as two-dimensional vanes, including the effects of streamtube thickness to determine the suction and pressure surface distributions of velocity, Mach number, static pressure, and static temperature. The vane loading, diffusion, and boundary layers are evaluated for acceptability, and the resulting $3 \mathrm{D}$ vane shape compared with the stage requirements.

Cylindrical endwalls ordinarily will be used with low-pressure-ratio turbine nozzles; contoured outer endwalls will be used on some first-stage nozzles. The preliminary subsonic-nozzle calculations for nozzle efficiency, outlet velocity, reheat, nozzle exit area, and tangential spacing use the same equations as the section for supersonic nozzles. The factors that affect supersonic nozzle performance, as discussed in the section above, apply to subsonic convergent-nozzle performance as well.

Partial-admission nozzles. Partial-admission nozzles are open for flow over only a fraction of the circumference of the turbine. The nozzles may be supersonic or subsonic, depending on the application. The minimum number of nozzles is one, but generally two or more nozzles will be used with an equal sector 180 deg apart and opposite each other, for structural and dynamic symmetry. As the number of sectors increases, losses are incurred due to the increasing number of transitions from the active to inactive arc of admission, as shown in Fig. 6-56.

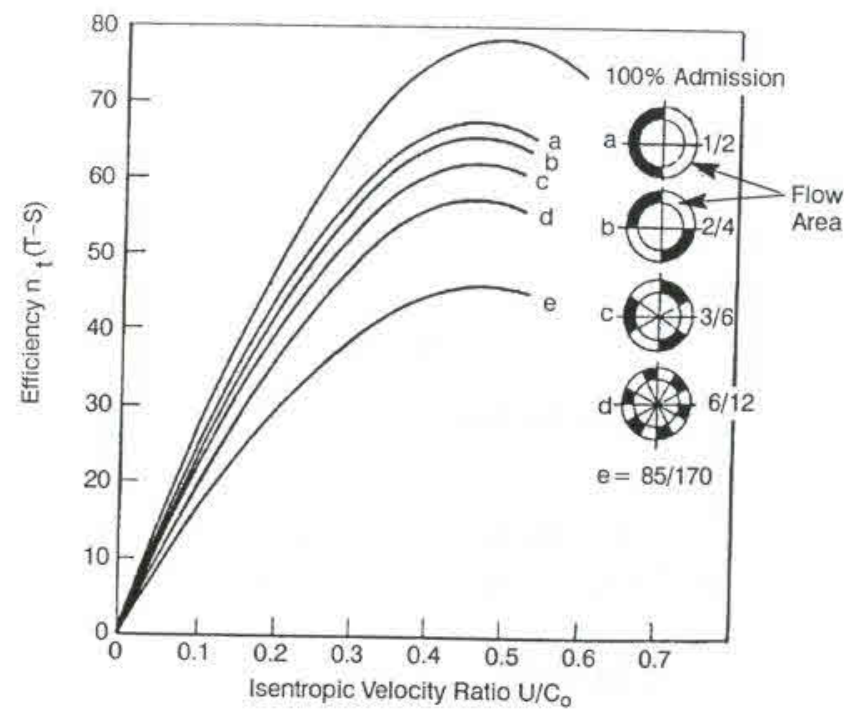

Fig. 6-56 Effect of number of active arcs on partial-admission turbine efficiency.

If the second-stage nozzle also has partial admission, the second nozzle can be set to capture the kinetic energy out of the first rotor at the design condition to maximize performance. An axial space upstream of the second nozzle will aid circumferential flow out of the first rotor into the second nozzle for off-design operation, such as startup and shutdown transients.

\section{Design of Turbine Rotor Blades}

Turbine rotor blades used in rocket-engine turbines can be classified as supersonic impulse blades and subsonic impulse and reaction blades.

Supersonic impulse rotor-blades. Rotor blades in an impulse turbine (Fig. 6-51 and 6-55) transform a maximum of the kinetic energy of the gases from the nozzles into useful work. Theoretically, there should be no change of gas static pressure, temperature, or enthalpy in the rotor blades. In actual operation, however, some gas expansion (i.e., reaction) usually occurs. Furthermore, the actual gas flow through the rotor blades deviates from ideal flow because of friction, eddy currents, boundary layers, and reheating.

The velocity-vector diagram shown in Fig. 6-55 describes graphically the flow conditions at the rotor blades of a single-stage, single-rotor turbine at the mean diameter $\mathrm{d}_{\mathrm{m}}$. The gases enter the rotor blades with an absolute velocity $C_{1}$ and at an angle $\alpha_{1}$ to the plane of rotation. The rotor blades have tangential or peripheral speed $U$ at the mean diameter. The relative velocities at the blade inlet and outlet differ (i.e., $V_{1}>V_{2}$ ) due to friction losses. Ideally, the gas should leave the blades at very low absolute velocity $\mathrm{C}_{2}$ and in a direction close to axial for optimum energy conversion in the blades. The forces generated at the rotor blades are a function of the change of momentum of the flowing gases. The following correlations can be established for design 
calculations of the rotor blades of a single-stage, single-rotor turbine.

Tangential force acting on the blades $(\mathrm{lb} / \mathrm{lb}$ of gas flow/s):

$$
\begin{aligned}
\mathrm{F}_{\mathrm{t}} & =\left(\mathrm{C}_{1} \cos \alpha_{1}+\mathrm{C}_{2} \cos \alpha_{2}\right) / \mathrm{g} \\
& =\left(\mathrm{V}_{1} \cos \beta_{1}+\mathrm{V}_{2} \cos \beta_{2}\right) / \mathrm{g}
\end{aligned}
$$

Work transferred to the blades ( $f t-l b$ per unit of gas flow $/$ s):

$$
\begin{aligned}
\mathrm{E}_{\mathrm{b}} & =\mathrm{U}\left(\mathrm{C}_{1} \cos \alpha_{1}+\mathrm{C}_{2} \cos \alpha_{2}\right) / \mathrm{g} \\
& =\mathrm{U}\left(\mathrm{V}_{1} \cos \beta_{1}+\mathrm{V}_{2} \cos \beta_{2}\right) / \mathrm{g} \\
\mathrm{U} & =\pi \mathrm{d}_{\mathrm{m}} \mathrm{N} / 720
\end{aligned}
$$

For subsequent calculations, the following relation will be useful:

$$
\tan \beta_{1}=C_{1} \sin \alpha_{1} /\left(C_{1} \cos \alpha_{1}-U\right)
$$

Axial thrust at blades (lb per unit of gas flow/s);

$$
\mathrm{F}_{\mathrm{a}}=\left(\mathrm{C}_{1} \sin \alpha_{1}-\mathrm{V}_{2} \sin \beta 2\right) / \mathrm{g}
$$

Blade velocity coefficient:

$$
\mathrm{k}_{\mathrm{b}}=\mathrm{v}_{2} / \mathrm{v}_{1}
$$

Blade efficiency:

$$
\eta_{\mathrm{b}}=\mathrm{E}_{\mathrm{b}} /\left(\mathrm{C}_{1} 2 / 2 \mathrm{~g}\right)
$$

Ideally, $\eta_{b}$ will be a maximum for a single-rotor impulse turbine when the turbine velocity ratio is-

$$
\mathrm{U} / \mathrm{C}_{1}=\frac{\cos \alpha_{1}}{2}
$$

i.e., when $\mathrm{U}=0.5 \mathrm{C}_{1 \mathrm{t}}$ where $\mathrm{C}_{1 \mathrm{t}}$ is the tangential component of $\mathrm{C}_{1}$. This yields a maximum ideal efficiency:

$$
\eta_{\mathrm{b}}=0.5 \cos ^{2} \alpha_{1}\left(1.0+\mathrm{k}_{\mathrm{b}} \cos \beta_{2} / \cos \beta_{1}\right)
$$

If there is some reaction or expansion of the gas flowing through the blades, the relative-gas-flow velocity at the rotor-blade outlet can be calculated as follows:

$$
\mathrm{v}_{2}=\left(\mathrm{k}^{2} \mathrm{v}_{1}^{2}+2 \mathrm{gJ} \eta_{\mathrm{n}} \Delta \mathrm{H}_{1-2^{\prime}}\right)^{0.5}
$$

Amount of rebeat in the rotor blades (Btu/lb of gas flow):

$$
\mathrm{qbr}_{\mathrm{br}}=\left(1.0-\mathrm{k}_{\mathrm{b}}{ }^{2}\right) \mathrm{V}_{1}{ }^{2 /(2 \mathrm{gJ})}+\left(1.0-\eta_{\mathrm{n}}\right) \Delta \mathrm{H}_{1-2^{\prime}}
$$

where-

$$
\alpha_{1}, \alpha_{2}=\text { absolute-gas-flow angles at the inlet }
$$
and outlet of the rotor blades, deg

$$
\begin{aligned}
& \beta_{1}, \beta_{2}=\text { relative-gas-flow angles at the inlet } \\
& \mathrm{C}_{1}, \mathrm{C}_{2}=\text { absolute-gas-flow velocities at the inlet } \\
& \text { and outlet of the rotor blades, } \mathrm{ft} / \mathrm{s} \\
& \mathrm{V}_{1}, \mathrm{~V}_{2}=\text { relative-gas-flow velocity at the inlet } \\
& \text { and outlet of the rotor blades, } \mathrm{ft} / \mathrm{s} \\
& \mathrm{U}=\text { peripheral speed of the rotor, } \mathrm{ft} / \mathrm{s} \\
& \mathrm{d}_{\mathrm{m}}=\text { mean diameter of the rotor, in } \text {. } \\
& \eta_{\mathrm{n}}=\text { equivalent nozzle efficiency appli- } \\
& \text { cable to the expansion process in the } \\
& \text { blades }
\end{aligned}
$$

All parameters refer to the mean diameter $d_{m}$ unless specified otherwise. The turbine overall efficiency $n_{t}$ defined by Eq. (6-15, 6-16) can be established for a single-stage, single-rotor impulse turbine in the following form:

$$
\eta_{\mathrm{t}}=\eta_{\mathrm{n}} \eta_{\mathrm{b}} \eta_{\mathrm{m}}
$$

where-

$\eta_{\mathrm{n}}=$ nozzle efficiency

$\eta_{\mathrm{b}}=$ rotor-blade efficiency

$\eta_{\mathrm{m}}=$ machine efficiency, indicating the mechanical, leakage, and disk-friction losses

Equation (6-84) shows that the blade efficiency $\left(\eta_{b}\right)$ improves when $\beta_{2}$ becomes much smaller than $\beta_{1}$. Reduction of $\beta_{2}$ without decreasing the flow area at the blade exit can be achieved through an unsymmetrical blade (Fig. 6-55), in which the radial blade height increases toward the exit. In actual designs, the amount of decrease of $\beta_{2}$, or the increase of radial height, will be limited by incipient flow separation and centrifugal stresses. Generally, the $\beta_{2}$ of an unsymmetrical blade will be approximately $\beta_{1}-(5$ to $15 \mathrm{deg}$ ). Equation (6-84) also indicates that $\eta_{\mathrm{b}}$ improves as $\alpha 1$ is reduced. Design values of $\mathrm{k}_{\mathrm{b}}$ vary from 0.80 to 0.90 . Design values of $\eta_{\mathrm{b}}$ range from 0.7 to 0.92 .

Referring to Fig. 6-55, the radial height at the rotor inlet $h_{\mathrm{b}}$ will usually be slightly larger $(5$ to $10 \%)$ than the nozzle radial height $h_{n}$. This height, together with the blade peripheral speed $U$, will determine the centrifugal stress in the blades. The mean diameter of the rotor blades is defined as $d_{m}$ $=d_{t}-h_{b}$ where $d_{t}$ is the rotor tip diameter. Pitch or blade spacing $\mathrm{P}_{\mathrm{b}}$ is measured at the mean diameter $\mathrm{d}_{\mathrm{m}}$. There is no critical relationship between blade pitch $\mathrm{P}_{\mathrm{b}}$ and nozzle pitch $\mathrm{P}_{\mathrm{n}}$. There should be a sufficient number of blades in the rotor to direct the gas flow. The number of blades $z_{b}$ to be employed is established by the blade aspect ratio $h_{b} / C_{b}$ and the solidity $\mathrm{C}_{\mathrm{b}} / \mathrm{P}_{\mathrm{b}}$, where $\mathrm{C}_{\mathrm{b}}$ is the chord length of the rotor blades. The magnitude of the blade aspect 
ratio ranges from 1.3 to 2.5 . Design values of blade solidity vary from 1.4 to 2 . Best results will be determined by experiment. The number of rotor blades should have no common factor with the number of nozzles or of stator blades for the purpose of minimizing dynamic loading.

The blade face is concave, with radius $\mathrm{r}_{\mathrm{f}}$. The back is convex, with a circular arc of small radius $r_{r}$ concentric with the face of the adjoining blade ahead. Two tangents to this arc to form the inlet; and outlet-blade angles $\theta_{\mathrm{b} 1}$ and $\theta_{\mathrm{b} 2}$ complete the blade back. The leading and trailing edges may have a small thickness $t_{b}$. Unsymmetrical-impulse-blade profiles have also been defined using tangent parabola curves and combinations of straight lines, circular arcs, and parabolas.

The inlet-blade angle $\theta_{\mathrm{b} 1}$ should be slightly larger than the inlet relative-flow angle $\beta_{1}$. If $\theta_{\mathrm{b} 1}<\beta_{1}$ the gas stream will strike the backs of the blades at the inlet, exerting a retarding effect on the blades and causing losses. If $\theta_{\mathrm{b} 1}>\beta_{1}$ the stream will strike the concave faces of the blades and tend to increase the impulse. The outlet-blade angle $\theta_{\mathrm{b} 2}$ is usually made equal to the outlet relative-flow angle $\left(\beta_{2}\right)$.

The mass flow rate $w_{t}$ through the various nozzle and blade sections of a turbine is assumed constant. The required blade flow areas can be calculated by the following correlation (Note that the temperature values used in calculating the gas densities at various sections must be corrected for reheating effects from friction and turbulence):

$$
\begin{aligned}
\dot{\mathrm{w}}_{\mathrm{t}} & =\rho_{1} \mathrm{~V}_{1} \mathrm{~A}_{\mathrm{b} 1} \varepsilon_{\mathrm{b} 1} / 144 \\
& =\rho_{2} \mathrm{~V}_{2} \mathrm{~A}_{\mathrm{b} 2} \varepsilon_{\mathrm{b} 2} / 144
\end{aligned}
$$

Total blade inlet area, in. 2 :

$$
\begin{aligned}
A_{b 1} & =z_{b} b_{b 1} h_{b 1} \\
& =z_{b} h_{b 1}\left(P_{b} \sin \theta_{b 1}-t_{b}\right)
\end{aligned}
$$

Total blade exit area, in. 2 :

$$
\begin{aligned}
A_{b 2} & =z_{b} b_{b 2} h_{b 2} \\
& =z_{b} h_{b 2}\left(P_{b} \sin \theta_{b 2}-t_{b}\right)
\end{aligned}
$$

where-

$$
\begin{aligned}
& \mathrm{P}_{\mathrm{b}}=\text { pitch or rotor-blade spacing } \\
& \rho_{1}, \rho_{2}=\text { density of gases at inlet and outlet of } \\
& v_{1}, v_{2}=\text { relative gas velocities at inlet and } \\
& \text { outlet of the rotor blades, } \mathrm{ft} / \mathrm{s} \\
& \varepsilon_{\mathrm{b} 1}, \varepsilon_{\mathrm{b} 2}=\text { area coefficients at inlet and outlet of } \\
& \mathrm{Z}_{\mathrm{b}}=\text { number of blades } \\
& h_{b 1}, h_{b 2}=\text { radial height at inlet and outlet of the } \\
& \text { rotor blades, in. } \\
& \mathrm{b}_{\mathrm{b} 1}, \mathrm{~b}_{\mathrm{b} 2}=\text { passage widths (normal to flow) at } \\
& \text { inlet and outlet of the blades, in. } \\
& \theta_{\mathrm{b} 1}, \theta_{\mathrm{b} 2}=\text { rotor blade angles at inlet and outlet, } \\
& \text { deg } \\
& t_{b}=\text { thickness of blade at inlet and outlet, }
\end{aligned}
$$
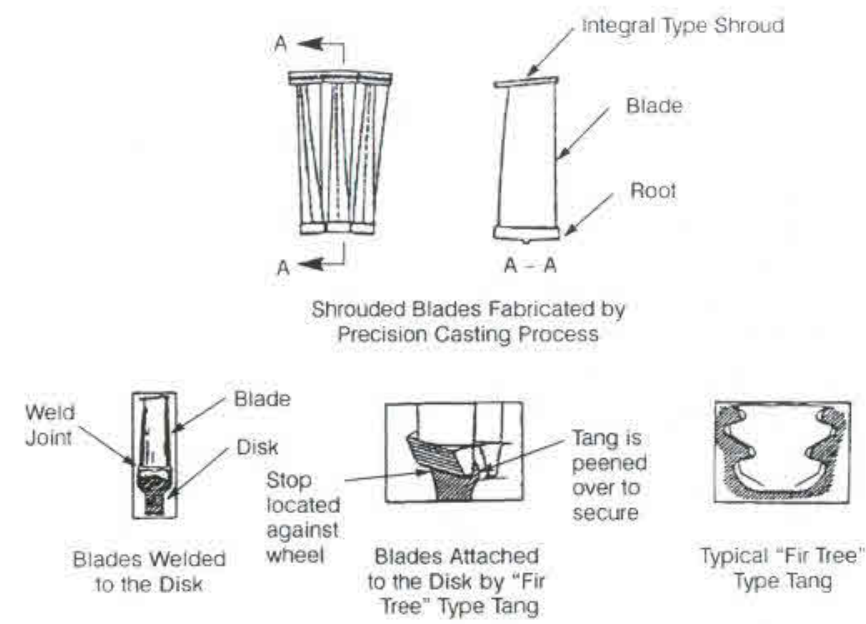

Fig. 6-57 Typical rotor-blade construction.

Typical construction of turbine rotor blades and disks are shown in Fig. 6-52, 6-51, 6-55, and 6-57. Usually, blades are designed with a shroud to prevent leakage over the blade tips and to reduce turbulence and thereby improve efficiency. The shroud also limits blade vibration amplitudes and increases rotor dynamic stability. Frequently the shroud forms an integral portion of the blade, the shroud sections fitting closely together when assembled. In other designs the shroud may form a continuous ring (Fig. 6-51) attached to the blades by tongues at the blade tip or by rivets. It can also be welded to the shrouds. The blades may be either welded to the disk or attached to it using "fir-tree" or other dovetail shapes.

The main loads experienced by a rotor blade can be divided into three types:

- Tension and bending due to centrifugal forces. The radial component of the centrifugal forces acting on the blade body produces a centrifugal tensile stress that reaches a maximum at the root section. As a remedy, blades are often hollowed and tapered, with the thinner section at the tip, to lower centrifugal root-stresses. The centroids of various blade sections at different radii generally do not fall on a true radial line. Therefore, the centrifugal forces acting upon the offset centroids will produce bending stresses that also are at maximum at the root section.

- Bending due to gas loading. The tangential driving force and the axial thrust produced by the momentum change of the gases passing over the blades produce bending stresses. These forces are sometimes integrated and treated as acting at the midheight of the blade to determine the amount of bending induced. Otherwise, distributed pressures on the blade are defined from a 3D flow analysis.

- Bending due to vibration loads. Gas does not flow uniformly in the blade passages, as assumed in theory, but varies cyclically from minimum to maximum. The resultant loads represent a dynamic force on the blades, having a corresponding cyclic variation. The frequency of this force coming equal to the natural frequency of the blades may cause deflections that will induce bending stresses of considerable magnitude. Therefore, the primary 
forcing functions acting on the blades are designed so as not to correspond in frequency with any blade natural frequency.

Detail stress analyses for rotor blades can be rather complex. A basic approach counteracts a major portion of the bending moments from gas loading with the bending moments induced by the centrifugal forces at nominal operating speeds. This can be done by careful blade design. Thus the centrifugal tensile stresses become a first consideration in blade design, while other details such as centroid location and root configuration are established later to fulfill other design requirements. The following correlations have been established at the blade-root section where stresses are most critical:

Centrifugal tensile stress at the root section of blade of a uniform cross section, psi:

$$
\mathrm{S}_{\mathrm{C}}=0.00548 \rho_{\mathrm{b}} \mathrm{h}_{\mathrm{b}} \mathrm{d}_{\mathrm{m}} \mathrm{N} 2 / \mathrm{g}
$$

Centrifugal tensile stress at the root section of tapered blade, psi:

$$
\begin{aligned}
\mathrm{S}_{\mathrm{ct}} & =0.00548 \frac{1}{\mathrm{~g}} \rho_{\mathrm{b}} \mathrm{h}_{\mathrm{b}} \mathrm{d}_{\mathrm{m}} \mathrm{N}^{2} \\
\times & {\left[1-\frac{1-\left(\frac{\mathrm{a}_{\mathrm{t}}}{\mathrm{a}_{\mathrm{r}}}\right)}{2}\left(1+\frac{\mathrm{h}_{\mathrm{b}}}{3 \mathrm{~d}_{\mathrm{m}}}\right)\right] }
\end{aligned}
$$

Bending moment due to root-section gas loading, in-lb:

$$
\mathrm{M}_{\mathrm{g}}=\mathrm{h}_{\mathrm{b}} \dot{\mathrm{w}}_{\mathrm{t}}\left(\mathrm{F}_{\mathrm{t}}^{2}+\mathrm{F}_{\mathrm{a}}{ }^{2}\right)^{0.5 / 2 \mathrm{z}_{\mathrm{b}}}
$$

where

$$
\begin{aligned}
\text { where } & =\text { gravitational constant, } \mathrm{ft} / \mathrm{sec}^{2} \\
\rho_{\mathrm{b}} & =\text { density of the blade material, } \mathrm{lb} / \mathrm{in} .3 \\
\mathrm{~h}_{\mathrm{b}} & =\text { average blade height, in. } \\
\mathrm{d}_{\mathrm{m}} & =\text { mean diameter of the rotor, in. } \\
\mathrm{N} & =\text { turbine speed, rpm } \\
\mathrm{a}_{\mathrm{r}} & =\text { sectional area at the blade root, in. }{ }^{2} \\
\mathrm{a}_{\mathrm{t}} & =\text { sectional area at the blade tip, in. }{ }^{2} \\
\dot{\mathrm{w}}_{\mathrm{t}} & =\text { turbine gas flow, } \mathrm{lb} / \mathrm{s} \\
\mathrm{z}_{\mathrm{b}} & =\text { number of blades } \\
\mathrm{F}_{\mathrm{t}} & =\text { tangential force acting on the blades, } \\
& \text { lb/lb/s } \\
\mathrm{F}_{\mathrm{a}} & =\text { axial thrust acting on the blades, } \mathrm{lb} / \mathrm{lb} / \mathrm{s}
\end{aligned}
$$

The bending stresses at the root can be calculated from the resultant bending moment and the blade-root section properties. The vibration stresses can be estimated from past design data. A shroud added to a blade increases stresses at the root. The total stress at the root section adds these stresses to those caused by the centrifugal forces acting on the blades.

The stresses in a turbine-rotor disk are induced by the blades and the centrifugal forces acting on the disk material itself. In addition, torque causes shear stresses. As seen in Fig. 6-51, turbine disks are generally quite thick at the axis, but taper off to a thinner disk rim to which the blades are attached. In single-rotor applications it is possible to design a solid disk so that both radial and tangential stresses are uniform at all points, shear being neglected. In multirotor applications, it is difficult to do this because of the greatly increased axial length and the resulting large gaps between rotor and stator disks.

Equation (6-94) may be used to estimate the average tangential stress in any turbine disk, neglecting rotor-blade effects:

$$
\mathrm{S}_{\mathrm{d}}=0.00137\left(\rho_{\mathrm{d}} / \mathrm{g}\right) \mathrm{d}_{\mathrm{d}^{2}} \mathrm{~N}^{2} /\left[\log _{\mathrm{e}}\left(\mathrm{t}_{\mathrm{o}} / \mathrm{t}_{\mathrm{r}}\right)\right]
$$

where-

$\mathrm{S}_{\mathrm{d}}=$ average tangential tensile stress of a constant-stress turbine disk, psi

$\rho_{\mathrm{d}}=$ density of the disk material, $\mathrm{lb} / \mathrm{in} \cdot 3$

$\mathrm{d}_{\mathrm{d}}=$ diameter of the disk, in .

$\mathrm{N}=$ turbine speed, $\mathrm{rpm}$

$t_{\mathrm{O}}=$ thickness of the disk at the axis, in.

$\mathrm{t}_{\mathrm{r}}=$ thickness of the disk rim at dd, in .

$\mathrm{g}=$ gravitational constant, $\mathrm{ft} / \mathrm{sec}^{2}$

Equation (6-95) permits estimation of the stresses in any turbine disk, neglecting effects of the rotor blades:

$$
\mathrm{S}_{\mathrm{d}}=0.00548 \mathrm{w}_{\mathrm{d}} \mathrm{r}_{\mathrm{i}} \mathrm{N}^{2} /\left(\mathrm{a}_{\mathrm{d}} \mathrm{g}\right)
$$

where-

$$
\begin{aligned}
\mathrm{S}_{\mathrm{d}}= & \text { centrifugal tensile stress of the turbine } \\
& \text { disk, psi } \\
\mathrm{W}_{\mathrm{d}}= & \text { weight of the disk, } \mathrm{lb} \\
\mathrm{r}_{\mathrm{i}}= & \text { distance of the center of gravity of the } \\
& \text { half disk from the axis, in. } \\
\mathrm{a}_{\mathrm{d}}= & \text { disk cross-sectional area, in }{ }^{2} \\
\mathrm{~N}= & \text { turbine speed, rpm } \\
\mathrm{g}= & \text { gravitational constant, } \mathrm{ft} / \mathrm{sec}^{2}
\end{aligned}
$$

For good turbine design, it is recommended that at the maximum allowable design rotating speed the $\mathrm{S}_{\mathrm{d}}$, calculated by Eq. (6-95), be about 0.75 to 0.8 the material yield-strength.

Turbine rotor blades and disks are made of hightemperature alloys of three different base materials: iron, nickel, and cobalt, with chromium forming one of the major alloying elements. High tensile strength and fatigue resistance at the operating temperature are important criterion for material selection. Other required properties include resistance to hydrogen embrittlement, low creep rate, and oxidation and erosion resistance. Astroloy, Waspaloy, Mar M-246, A 286, and Inconel 718 are alloys frequently used. The rotor blades are fabricated either by precision casting or by precision forging. Rotor disks are best made of forgings for optimum strength. Integrallybladed disks ("blisks") are also used, but blade damping is more difficult to achieve, requiring more care to avoid fatigue damage.

Subsonic Blades. The design of subsonic rotor blades uses extensive, interactive computer graphics and computer-aided engineering (CAE). The design 
process, depicted in Fig. 6-53, sets the basic requirements of each blade row by a meanline-gaspath analysis. The turbine-configuration optimization follows various constraints. The rotor profile will be defined by a multistage meridional-flow-field analysis for the specified blade-path elevation. The results of the meridional analysis establish the acceleration and turning characteristics of each rotor-profile section from hub to tip. Initial rotor profiles are generated on the computer at several radial locations. The initial profile surface-velocity and static-pressure distributions can be checked using estimated streamtube thicknesses for adverse pressure gradients based on established guidelines. The rotor profiles are stacked from hub to tip, as can be seen in Fig. 6-54, including an estimate of the lean and tilt that may be required from the structural design to establish the 3D blade profile.

The 3D geometry is used in an inviscid multistage quasi-3D or full-3D analysis program that determines the streamline and blade-to-blade flowfield characteristics. The multistage capability being required, a complete evaluation can be made of potential changes of individual blade surfaces, including the effects on the other turbine-blade rows. The profile geometry along a specified streamline will be determined so that detailed suction and pressure surface distributions can be calculated using a suitable flow-code. These distributions will be used in the boundary-layer code to determine the boundary-layer characteristics that are assessed for losses and separation potential. Solid modeling of the defined 3D blade path that can be viewed from any orientation allows an early look at the turbine blading for mechanical, structural, and thermal design analyses.

\section{Design of Single-Stage, Two-Rotor, Velocity- Compounded Impulse Turbines}

Most impulse turbines will have only one or two rotors. It is assumed that a single-stage, two-rotor, velocity-compounded impulse turbine expands gases completely in the nozzle, and that no further staticpressure change occurs during gas flow through the moving blades. As mentioned earlier, the two-rotor, velocity-compounded arrangement provides high efficiency at low velocity ratios. In this case, the gases ejected from the first rotor blades still possess considerable kinetic energy. They are therefore redirected by a row of stationary blades into a second row of rotor blades that extract additional work from them. The gases usually leave the second rotor-blade row at a moderate velocity and in a direction close to axial.

The velocity diagrams of a single-stage, tworotor, velocity-compounded impulse turbine are based on a mean rotor diameter, as shown in Fig. 658 , the peripheral speed of the rotor blades at the diameter being $U$. The gases leave the nozzles and enter the first rotor blades with an absolute velocity $C_{1}$, at an angle $\alpha_{1}$ with the plane of rotation; $V_{1}$ and $\mathrm{V}_{2}$ are the relative flow velocities in $\mathrm{ft} / \mathrm{s}$ at the inlet and outlet of the first rotor blades. The gases leave the first rotor blades and enter the stationary blades at an absolute flow velocity $\mathrm{C}_{2}$, and at an angle $\alpha_{2}$. After passing over the stationary blades, the gases enter the second rotor blades at an absolute flow velocity $C_{3}$, and at an angle $\alpha_{3} ; V_{3}$ and $V_{4}$ are the relative inlet and outlet flow velocities at the second rotor blades. Angles $\beta_{1}, \beta_{2}, \beta_{3}$, and $\beta_{4}$ represent the flow directions of $\mathrm{V}_{1}, \mathrm{~V}_{2}, \mathrm{~V}_{3}$, and $\mathrm{V}_{4}$.

As with single-rotor turbines, the exit velocity from any row of blades (rotary or stationary) will be less than the inlet velocity because of friction losses. It can be assumed that the blade velocity coefficient $\mathrm{k}_{\mathrm{b}}$ has the same value for any row of blades:

$$
\mathrm{k}_{\mathrm{b}}=\mathrm{V}_{2} / \mathrm{V}_{1}=\mathrm{C}_{3} / \mathrm{C}_{2}=\mathrm{V}_{4} / \mathrm{V}_{3}
$$

In a multirotor turbine, the total work transferred will be the sum from the individual rotors:

Total work transferred to the blades of a two-rotor turbine, $\mathrm{ft}-\mathrm{lb} / \mathrm{lb}$ of gas flow/s:

$$
\begin{aligned}
E_{2 b}= & \frac{U}{g}\left(C_{1} \cos \alpha_{1}+C_{2} \cos \alpha_{2}+C_{3} \cos \alpha_{3}+C_{4} \cos \alpha_{4}\right) \\
= & \frac{U}{g}\left(V_{1} \cos \beta_{1}+V_{2} \cos \beta_{2}+V_{3} \cos \beta_{3}\right. \\
& \left.+V_{4} \cos \beta_{4}\right)
\end{aligned}
$$

Combined nozzle and blade efficiency of a tworotor turbine:

$$
h_{n b}=E_{2 b} /(J \Delta H)
$$

where $\Delta H=$ overall isentropic enthalpy drop of the turbine gases $(\mathrm{Btu} / \mathrm{lb})=$ total available energy content of the turbine gases (Eq. 6-13). Equation (6-87) can be rewritten for the turbine overall efficiency $\eta_{t}$ of a two-rotor turbine as follows:

$$
\eta_{\mathrm{t}}=\eta_{\mathrm{nb}} \eta_{\mathrm{m}}
$$

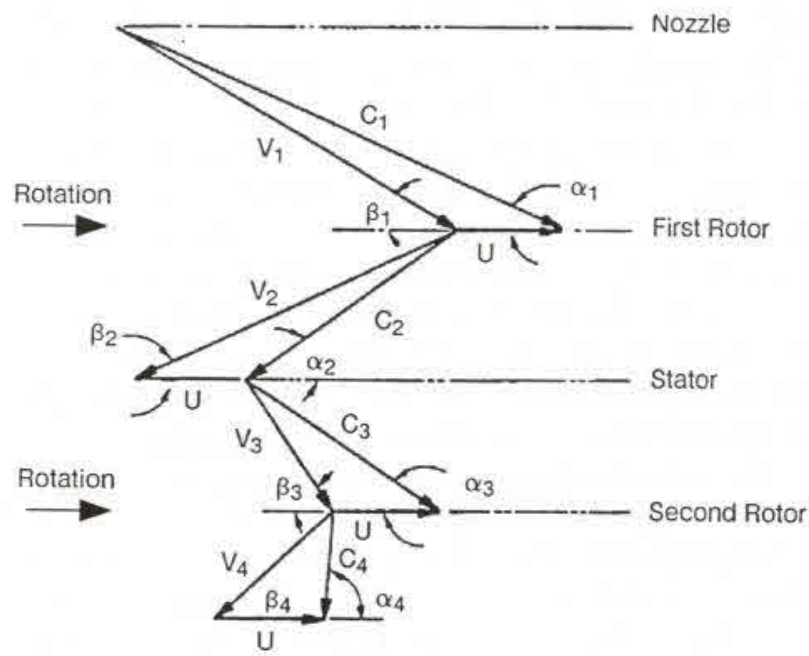

Fig. 6-58 Velocity diagrams of a typical singlestage, two-rotor, velocity-compounded impulse turbine. 
Ideally, $\eta_{\mathrm{b}}$ would be a maximum for the single-stage, two-rotor, velocity-compounded impulse turbine velocity ratio:

$$
\mathrm{U} / \mathrm{C}_{1}=0.25 \cos \alpha_{1}
$$

i.e., when $\mathrm{U}=0.25 \mathrm{C}_{1 \mathrm{t}}$. The workload for the second rotor of a two-rotor, velocity-compounded turbine is designed at about one-fourth of the total work.

The design procedures for the gas-flow passages of the rotor and stationary blades of a single-stage, two-rotor turbine are exactly the same as those for a single-rotor turbine. However, velocities and angles of flow change with each row of blades. As a result, the radial height of symmetrical blades increases with each row, approximately as shown in Fig. 6-51. The effects of reheating (increase of gas specific volume) in the flow passages must be taken into account when calculating the gas densities at various sections. Equation (6-86) may be used to estimate the amount of reheat at each row of blades.

In the calculations for multi-row unsymmetrical blades, the radial heights at the exit side of each row are determined first by Eq. (6-90). The radial heights at the blade inlets are then made slightly larger, by approximately $8 \%$, than those at the exit of the preceding row.

\section{Design of Two-Stage, Two-Rotor Pressure- Compounded Impulse Turbine}

An operational schematic of a typical two-stage, tworotor, pressure-compounded impulse turbine and its velocity diagrams at the mean diameter are shown in Fig. 6-12 and 6-59. Each stage of a pressure-compounded impulse turbine may be regarded as a single-stage impulse turbine rotating in its own individual housing. Most of the design characteristics of a single-stage turbine are applicable to the individual stages. The gas-spouting velocities $\mathrm{C}_{1}$ and $\mathrm{C}_{3}$, at flow angles $\alpha_{1}$ and $\alpha_{3}$, of the first- and secondstage nozzles are designed to be approximately the same; $V_{1}, V_{2}, V_{3}$, and $V_{4}$ represent the relative flow velocities at inlets and outlets of the rotor blades; $\beta_{1}$, $\beta_{2}, \beta_{3}$, and $\beta_{4}$ are the corresponding flow angles for $\mathrm{V}_{1}, \mathrm{~V}_{2}, \mathrm{~V}_{3}$, and $\mathrm{V}_{4}$. The second-stage nozzles are designed to receive the gas flow discharged from the first-stage rotor blades at an absolute velocity $\mathrm{C}_{2}$, and to turn it efficiently to a desired angle $\alpha_{3}$. Simultaneously, by expansion to a lower pressure, the gases are accelerated to a desired velocity $\mathrm{C}_{3}$. Flow at the outlet of the second rotor has an absolute velocity $\mathrm{C}_{4}$ and flow angle $\alpha_{4}$; $\mathrm{U}$ is the rotor peripheral speed at the mean effective diameter $d_{m}$.

The total work performed in the turbine sums the separate stages. These may be designed to divide the load equally (i.e., the velocity diagrams of each stage will be identical: $C_{1}=C_{3}, C_{2}=C_{4}, \alpha_{1}=\alpha_{3}, \alpha_{2}=\alpha_{4}$, etc.). The friction losses occurring in the first stage are passed on in the gas stream as additional enthalpy and increase the available energy for the second stage. Also, the kinetic energy of the gases leaving the first stage will not be entirely lost, as with

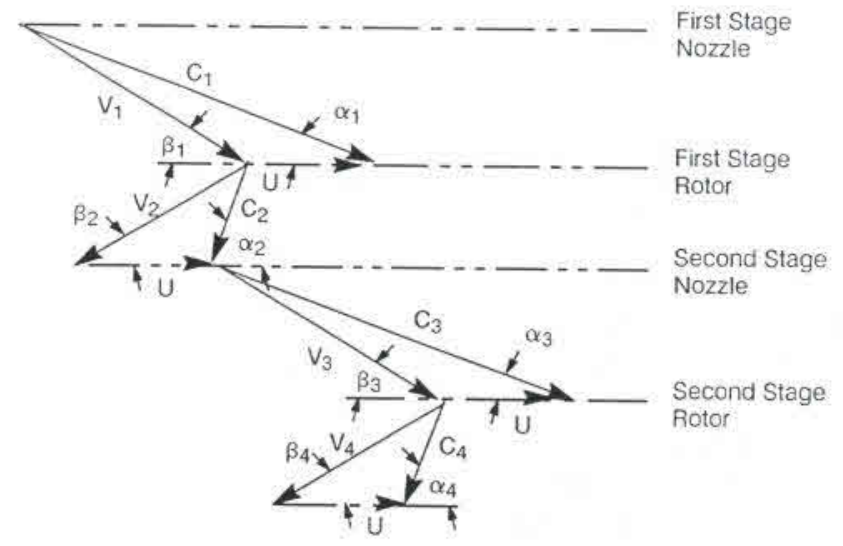

Fig. 6-59 Velocity diagrams of a typical two-stage, two-rotor, pressure-compounded impulse turbine.

a single-stage turbine. The carryover ratio $r_{C}$-i.e., the ratio of the kinetic energy actually utilized as inlet energy by the second-stage nozzles to the total kinetic energy of the gases leaving the first stagecan vary from 0.4 to close to 1.0 . The axial distance between the first-stage rotor and the second-stage nozzle, as well as the leakages through the sealing diaphragm between stages, should be minimized for optimum carryover.

Determining the correct enthalpy drop resulting in equal work for each stage may require a trial-anderror approach, in relation to the effects of reheating, or the proper enthalpy drop may be estimated from previous designs and test data. With the velocity coefficients for nozzles and blades given by past or concurrent experiments, Eq. (6-72) and (686) can be used to estimate the amount of reheating.

Most equations established for the single-stage turbines may be used in the design calculations for two-stage turbines. The following additional correlations are available for the design of choked second-stage nozzles:

$$
\begin{gathered}
\mathrm{T}_{2 \mathrm{t}}=\mathrm{T}_{2}+\mathrm{r}_{\mathrm{c}} \frac{\mathrm{C}_{2}^{2}}{2 \mathrm{gJC} \mathrm{C}_{\mathrm{p}}} \\
\mathrm{P}_{2 \mathrm{t}}=\mathrm{P}_{2}\left(\frac{\mathrm{T}_{2 \mathrm{t}}}{\mathrm{T}_{2}}\right)^{\frac{\gamma}{\gamma-1}} \\
\mathrm{C}_{3}=\mathrm{k}_{\mathrm{n}} \sqrt{2 \mathrm{gJC}_{\mathrm{p}} \mathrm{T}_{2 \mathrm{t}} \sqrt{\mathrm{r}_{\mathrm{c}} \mathrm{C}_{2}^{2}+2 \mathrm{gJ \Delta \textrm {H } _ { 2 - 3 }}}} \\
\left(\mathrm{A}_{\mathrm{nt}}\right)_{2}=\frac{\left.\left.\dot{\mathrm{p}}_{3}\right)^{\frac{\gamma-1}{\gamma}}\right]}{\epsilon_{\mathrm{nt}} \mathrm{P}_{2 \mathrm{t}} \sqrt{\frac{\mathrm{g} \gamma\left[\frac{2}{\gamma+1}\right]^{\frac{\gamma+1}{\gamma-1}}}{\mathrm{RT}_{2 \mathrm{t}}}}}
\end{gathered}
$$




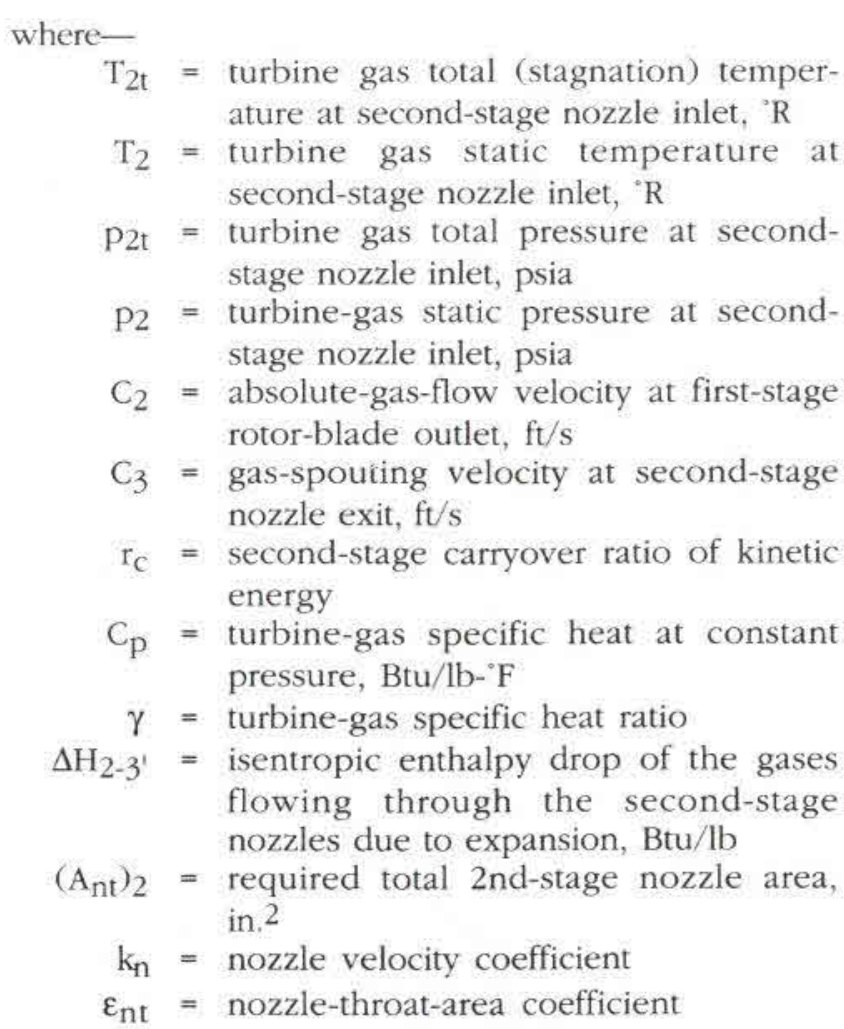

\section{Design of Two-Stage, Two-Rotor, Low-Reaction Turbine}

Two-stage, two-rotor, low-reaction turbines are used in staged-combustion-cycle or series-expander-cycle rocket engine systems to provide the highest turbine efficiency. In these engine systems, the turbine pressure ratio is low (1.5 to 2.0) and the stage velocity ratio is high ( 0.4 or higher). In highchamber-pressure oxygen/ hydrogen engines like the SSME, using two stages increased blade height, gave higher efficiency, and divided the high power between two rotating blade rows, lowering blade stresses in comparison with a single-stage turbine

The design calculation and resulting vector diagrams of a two-stage, low-reaction turbine (see Fig. 6-13) are similar to those for the two-stage, pressurecompounded impulse turbine (Fig. 6-12) discussed in the previous section (6.6.5), except for greater stage reaction (increased rotor-static-pressure drop fraction) and lesser nozzle and rotor turning. Twostage, low-reaction turbines usually optimize for nearly equal power split between the rotors, with the first stage producing the slightly higher power. The blading is usually twisted to minimize inlet incidence losses for the significant radial-flow-angle variations from hub to tip. Rotor-outlet absolute velocities are usually near axial (zero swirl). Reaction turbines have higher rotor axial thrust due to the higher static-pressure drop across the rotor.

Figure 6-60 shows the mean diameter velocity vector diagram for the SSME HPFTP turbine at full power level. Note the proportions of blade speed to nozzle-outlet velocity and relative velocity increase across the rotors compared with Fig. 6-59 for the pressure-compounded impulse turbine.

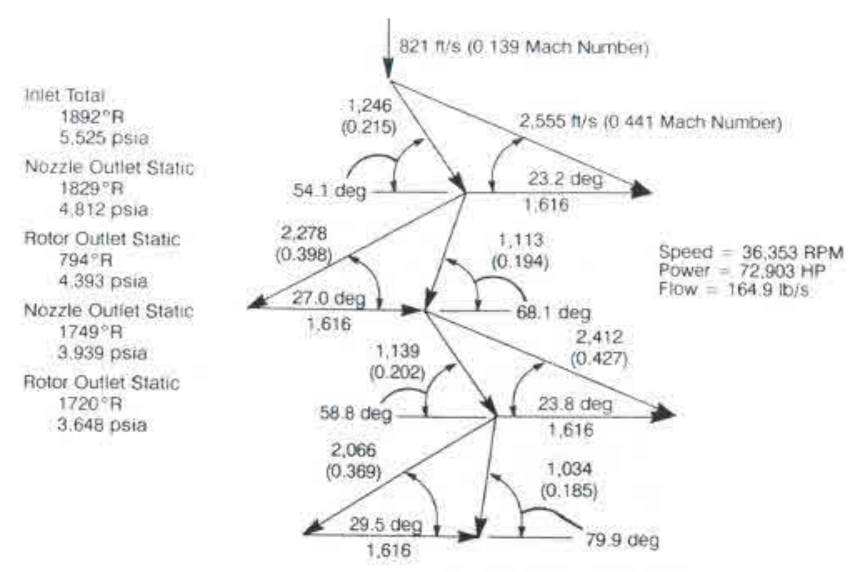

Fig. 6-60 SSME HPFTP turbine velocity diagram at full power.

\subsection{TURBOPUMP ROTORDYNAMICS AND MECHANICAL ELEMENTS}

\section{Rotordynamics}

The rotor structure will resonate when excited at its natural frequency. With the pump propellants used, the amount of damping obtained in a rocket engine turbopump is typically too small to critically damp the system. Therefore, at the resonance, large vibration amplitudes can be experienced and generally must be avoided. The rotating-assembly natural frequency will be dictated by the rotor mass and bending stiffness distribution coupled with the bearing-support-structure stiffness, and will include the gyroscopic effect of the spinning disks. "Rotor synchronous motion" means rotation with the shaft spin speed and precessional speed equal in direction and magnitude. The most common cause of forward synchronous motion is rotor unbalance. Rotor vibration amplitude is determined by the proximity of the natural frequency to the synchronous excitation and the amount of damping present in the system. Figure 6-61 shows how these factors influence the behavior of a simple rotor.

Operation of turbomachinery at or near a natural frequency causes excessive response, increases wear, and reduces reliability. Synchronous motion coincident with the rotor natural frequency is defined as operation at a critical speed. The dynamic response can be reduced by increasing the effective damping or designing the rotor to locate its natural frequencies far from the shaft-spin-speed excitation.

In dynamic analysis of the rotating assemblyan essential part of the turbopump design processcritical speeds, rotor-stability margins, bearing loads, shaft loads, and shaft deflections will be calculated to ensure that rotor-dynamic characteristics will be compatible with operating speed and transientresponse requirements when traversing critical speeds. Additional analyses are performed to investigate potential problem areas due to nonlinearities resulting in nonsynchronous shaft whirl or shaft loads and deflections due to steady-state hydrodynamic sideloads. Turbopump rotors are designed 
so that the critical speeds are well separated from the operating range. The rotor bearing supports are designed to provide the stiffness required to separate the critical speeds from the operating range and minimize the dynamic bearing loads and shaft deflections. The most desirable situation is to design the rotating assembly to operate below the first critical speed; that produces minimal bearing loads and shaft deflections even in the transients. A $20 \%$ separation margin between calculated critical speeds and operating speeds follows standard design practice, as demonstrated successfully in pump-fed rocket engines. Torsional vibration is not normally a problem in direct-drive turbopumps due to lack of a significant forcing function and sufficient damping.

Most turbomachinery rotors are a complex arrangement of components mounted on a shaft of variable diameter. The relationship between the rotor stiffness, mass, and damping distribution combined with gyroscopic effects determines the rotordamped natural frequencies and associated mode shapes. The mode shape is the relative rotor deformation present when operating at a natural frequency. Every natural frequency has a unique mode shape associated with its resonant vibration. Rotor mode shapes provide valuable information that graphically indicate factors that are influential in determining its characteristics, and, thus, provide guidance as to what design changes would be most effective in changing the natural frequencies away from the operating point.

Figure 6-62 graphically displays how physical change to the rotor supports can alter rotatingmachinery critical speeds and associated mode shapes. It is apparent from this data that support stiffness is a very important rotor-dynamic parameter. Design revisions will be necessary if inadequate critical-speed margins are present. Review of the rotor mode shapes can identify design modifications. In general, modifications to bearing and seal stiffness and damping can be effective if significant relative motion exists at that location. Modifications to the shaft bending stiffness can be effective if sufficient shaft angulation exists at the change location for the mode in question. Modifications to the mass distribution will only be effective when significant relative motion exists at the mass location to be relocated. Common practice maximizes the stiffness and damping and locates the bearings as close as possible to large rotor masses.

In addition to bearing and seal stiffness and damping as a function of shaft speed, dampednatural-frequency analyses include variable hydrodynamic influences. The damped critical speeds are identified as the coincidental occurrence of these natural frequencies and the shaft spin speed, as shown in Fig. 6-63.

These damped critical speeds and associated mode shapes are not only important in defining the safe-operating-speed margin, but also in predicting rotor stability. Most rotor-dynamic motion will either decay with time or maintain a constant level after application of an external excitation (perturbation) - evidence of a stable system. Unstable rotor

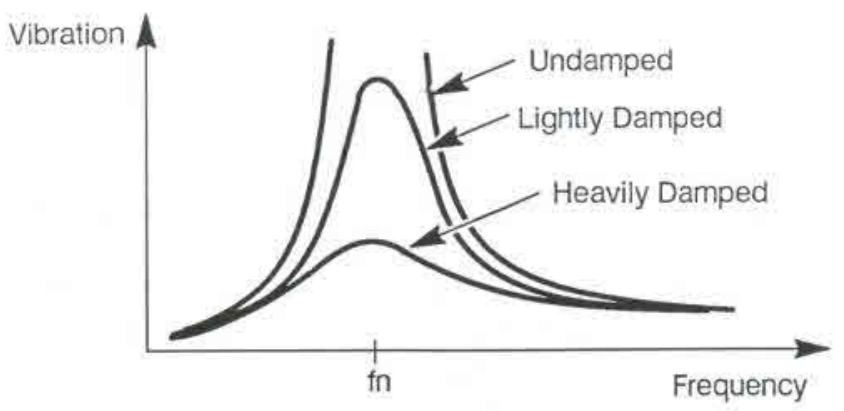

Fig. 6-61 Dynamic response of a simple system.

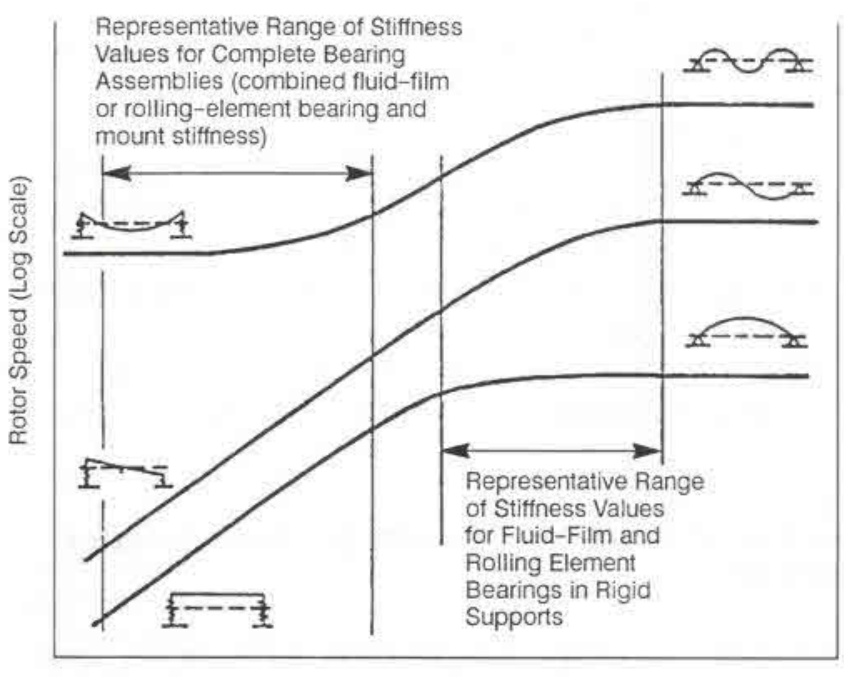

Rotor Support Stiffness (Log Scale)

Fig. 6-62 Effect of rotor supports on critical speeds.

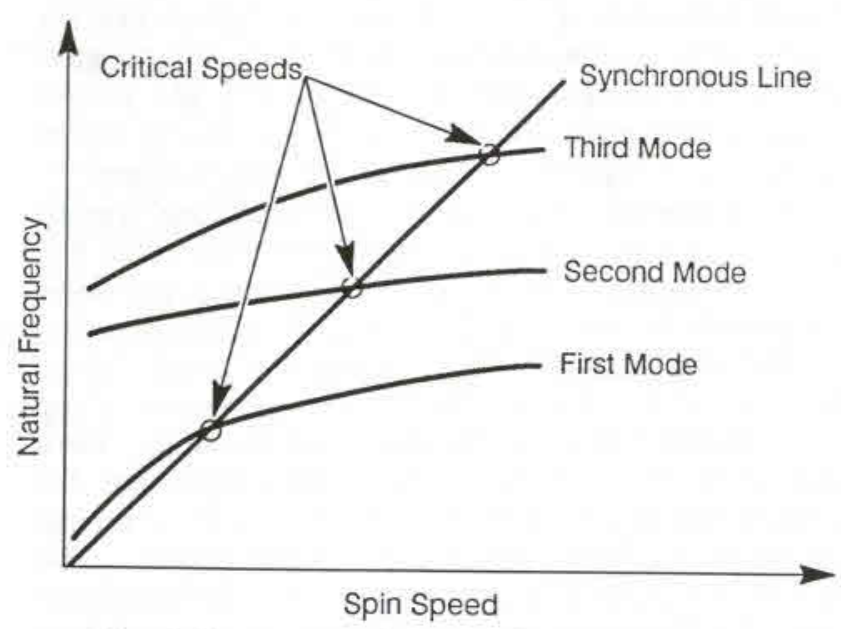

Fig. 6-63 Damped critical speed map.

assemblies will increase in response amplitude without bound after a perturbation, as shown in Fig. 6-64.

The most common form of rotor-dynamic instability, subsynchronous whirl, finds the rotor precessional speed less than the spin speed and coincident with a rotor natural frequency. This situation without sufficient damping can produce a vibration that may result in destruction or catas- 
trophic failure. Rotor instability exhibits a threshold spin speed at which the normal rotor response will increase without bound. The design goal is to maintain the threshold speed above the operating speed with a large margin.

Rotordynamic stability is controlled by providing an abundance of damping to suppress the destabilizing whirl drivers. These instability drivers produce tangential forces to the radial motion that oppose the damping between the rotor and housing. The tangential forces-commonly referred to as "cross-coupling" forces-can be in the direction of shaft rotation or counter to the rotation.

The most common destabilizing tangential forces that excite nonsynchronous rotor motion are the following:

Hydrodynamic forces under pump partial-cavitation

Tangential blade-tip forces

Annular seal forces due to Couette flow

Nonlinear load-deflection characteristics in bearings

Frictional forces between shafts and shrink-fit parts

Tangential rubbing forces acting on the rotor

Hydrodynamic loads on the rotor that rotate relative to the rotor

Hysteresis forces within the rotor

Coupling of unbalance forces from one rotor through the engine structure to another rotor

Common design techniques minimize crosscoupling forces and enhance support stiffness and damping. Hydrodynamic forces are primarily functions of blade and flow-path design, but rotordynamic performance is generally improved by the addition of shrouds. Friction forces can be controlled by avoiding the use of long-length shrink fits on the shaft. For instance, if a long spacer is required, it should not be shrunk-fit on the shaft along its entire length. Coupling of unbalance forces through the engine structure is not normally a problem and can be controlled by proper turbopump mount-design. Close-clearance annular shaft seals can be detrimental, but can also be used to significant rotor-dynamic benefit. Labyrinth seals provide minimal stiffness and damping, and under certain conditions can provide negative stiffness and stability concerns. Rotor-dynamic characteristics of these seals can be improved significantly by converting to smooth annular seals, possibly with a slight convergent taper to the stator bore. The seal coefficients can also be further enhanced generally with the addition of fluid anti-rotation ribs at the seal
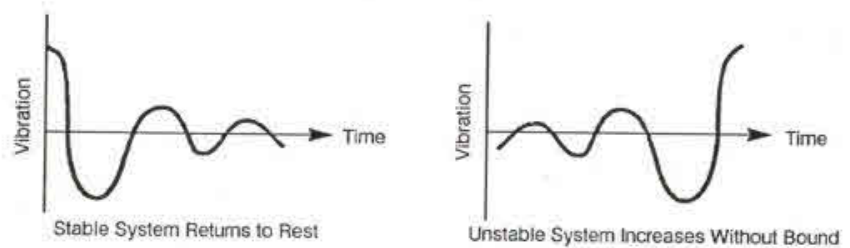

Fig. 6-64 Stability of a simple rotor system. entrance or roughening the seal bore relative to the shaft journal. The bearings provide a relatively large source of stiffness as the main rotor support. Rolling-element bearings tend to provide a nonlinear support stiffness, because clearance between the bearing outer diameter and housing will be made to meet other functional requirements during transient axial-shaft excursions. This nonlinearity might produce nonsynchronous rotor motion at a limited amplitude, which is usually not detrimental. Hydrostatic bearings can provide superior stiffness and damping as a rotor support and, when located optimally along the rotor, can critically damp the rotor-dynamic response.

The rotor stability is controlled by designing significant margin into the design. Rotor-dynamic analysis, including the various instability drivers and dampers along the rotating assembly, will predict the most unstable rotor mode. It usually will be the lowest mode because it usually has the least damping. Operating at twice the lowest mode is a common concern because seal tangential forces are near half shaft speed and are therefore potentially coincident with the lowest mode in this situation. Sufficient damping is required to preclude instabilities, and adequate margins should be maintained. Normal design practice avoids operation within $20 \%$ of the calculated stability-threshold speed. Additionally, rotor stability is controlled by providing sufficient damping at the operating speed to ensure a rapid vibration decay after a perturbation as a measure of the system safety margin. The "log-decrement" parameter quantifies the relative rate of vibration decay as a dimensionless number, allowing turbopumps of different size, weight, performance, etc. and different rotor modes all to be compared on an equal basis. The larger the log-decrement number, the more stable the system. At the threshold speed the log decrement equals zero, and a critically-damped rotor mode has a log decrement of 0.7 . It is debatable what log decrement should be maintained as suitable; but successful turbopump rotor experience dictates the log decrements for all modes at all rotational speeds within the operating range be at least 0.05 . Physically, this means that a vibration response after a perturbation will decay to half the initial response amplitude in 20 revolutions of the shaft because the reciprocal of the log decrement indicates the number of cycles decayed in one time-constant. For clarity, Fig. 6-65 shows a graphical representation of the rotor-stability evaluation.

In general, the rotor-dynamic analysis guides the mechanical arrangement of the various rotor components on the shaft to provide a rotating assembly with minimal dynamic response, thus arguing a long service life.

\section{Turbopump Bearing Design}

A turbopump shaft is supported by two or more bearings designed to transmit all the loads acting on the rotating members to the housing (Ref. 6-9). The loads acting on the rotating members can be divided into two classes: radial acting normal to the 


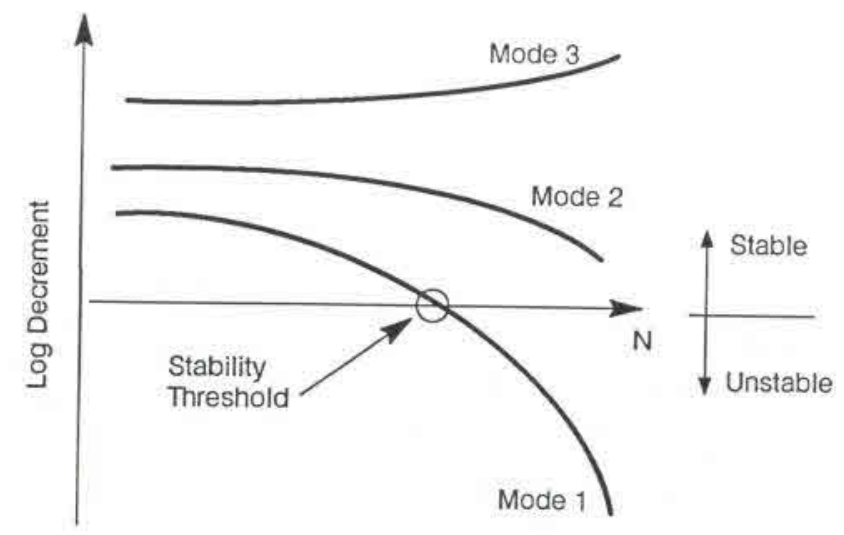

Fig. 6-65 Graphical representation of rotor stability. shaft axis and axial acting parallel to the shaft axis. In some cases, the radial loads also act to produce a tangential moment on the shaft that can be either in the direction of rotation (e.g., the tip-seal forces on turbines) or opposite the direction of rotation (e.g., the damping achieved by certain seal designs). The objective of the turbopump designer is to define any resultant loads that must be carried by the bearings and to minimize these over the operating range.

Rolling-element bearings have been used most extensively in rocket-engine turbopumps. These include both ball bearings, as seen in Fig. 6-4, and roller bearings, as seen in the turbine end of Fig. 6-9. The ball bearings can also be used as duplex pairs, as shown in Fig. 6-5. Rolling-element bearings have been used because of their high load capability independent of speed, high radial stiffness, ability to start and shut down reliably, and compatibility with the propellant fluids being used. Normally, these bearings operate directly in the propellant medium, the propellant serving to cool the bearing by a positive controlled flow. However, some propellants

Table 6-6 Comparison of turbopump rolling-element bearings.

\begin{tabular}{|c|c|c|}
\hline $\begin{array}{l}\text { Type of } \\
\text { Bearing }\end{array}$ & Advantages & Disadvantages \\
\hline $\begin{array}{l}\text { Conrad-type } \\
\text { ball }\end{array}$ & $\begin{array}{l}\text { Any combination of } \\
\text { radial and thrust } \\
\text { direction; large } \\
\text { misalignment capa- } \\
\text { bility; moment-load } \\
\text { capacity }\end{array}$ & $\begin{array}{l}\text { Limited number of } \\
\text { balls; two-piece } \\
\text { cage necessary }\end{array}$ \\
\hline $\begin{array}{l}\text { Angular- } \\
\text { Contact ball }\end{array}$ & $\begin{array}{l}\text { Thirty percent more } \\
\text { capacity than simi- } \\
\text { lar-size Conrad; one- } \\
\text { piece cage }\end{array}$ & $\begin{array}{l}\text { Predominant thrust } \\
\text { required; one- } \\
\text { direction capacity; } \\
\text { lower misalignment } \\
\text { tolerances than } \\
\text { Conrad }\end{array}$ \\
\hline Split-ring & $\begin{array}{l}\text { Thirty percent more } \\
\text { thrust capacity than } \\
\text { similar-size Conrad; } \\
\text { one-piece cage; two- } \\
\text { direction thrust } \\
\text { capability; lower } \\
\text { axial clearance through } \\
\text { use of Gothic arch }\end{array}$ & $\begin{array}{l}\text { Predominant thrust } \\
\text { required; lower mis- } \\
\text { alignment tolerance } \\
\text { than Conrad }\end{array}$ \\
\hline Cylindrical & $\begin{array}{l}\text { Much higher radial } \\
\text { capacity than ball } \\
\text { bearing; provides } \\
\text { axial freedom of } \\
\text { shaft; higher radial } \\
\text { stiffness than ball } \\
\text { bearings; one-piece } \\
\text { cage }\end{array}$ & $\begin{array}{l}\text { No axial load } \\
\text { capacity; roller ends } \\
\text { wear in nonlubrica- } \\
\text { ting coolants; } \\
\text { lower misalignment } \\
\text { tolerance than ball } \\
\text { bearings; requires } \\
\text { negative internal } \\
\text { clearance }\end{array}$ \\
\hline
\end{tabular}

\section{Primary condition for use}

Combined load; twodirection thrust loads

High speed, high-load single-direction thrust; can be used in duplex pairs for two-direction thrust

Two-direction thrust

High radial capacity without axial restraint; higher radial stiffness 


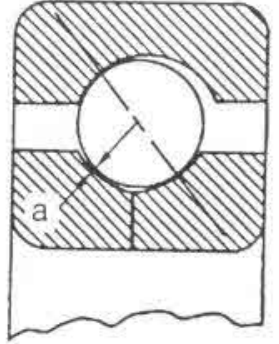

Normal Operation (Clearance at a)

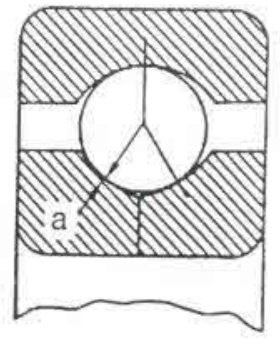

Three-Point Contact (Wiping Contact at a)
Split-Inner-Ring Ball Bearing

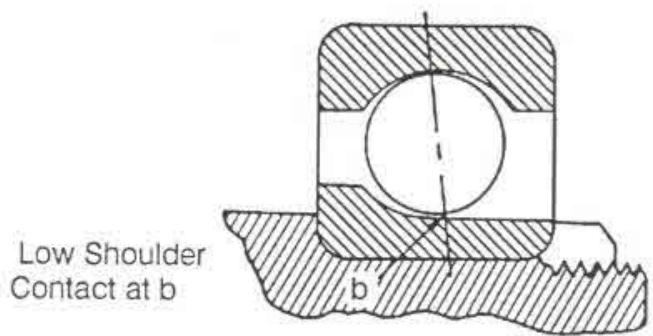

Angular-Contact Ball Bearing Fig. 6-66 Typical ball-bearing designs.

do not provide lubrication to the bearing and, as a result, allow bearing wear or surface distress. For many turbopump applications, the wear rate is sufficiently low that the resulting life of the rollingelement bearing more than adequately meets the turbopump requirements. Such bearings have been used very successfully in turbopumps for expendable launch vehicles.

To overcome the limitations of rolling-element bearings for long-life high-speed turbopumps, hydrostatic bearings are being introduced. These bearings operate on a fluid film and derive their ability to sustain loads and provide required rotor-dynamic stiffness through a high-pressure supply of the propellant from the pump discharge. These bearings also offer basically unlimited speed and positive damping to the turbopump rotor. There-fore, they are of particular value when high speeds are desirable and operation above one or more critical speeds is desired. Primary limitation: they must start and shut down in a rubbing contact. The ability to do this successfully has been demonstrated for certain applications and is the subject of ongoing technology developments.

Hybrid systems consisting of both rolling element and hydrostatic bearings can also be used where the rolling-element bearing carries the loads at start and shutdown when the hydrostatic pressure for the bearing is low. As pump speed increases, the hydrostatic pressure increases, and the hydro-static bearing begins to take an ever-increasing percentage of the applied loads because of its larger stiffness. Thus, the life of the rolling-element bearing is extended because it carries low loads at speed, and the life of the hydrostatic bearing is extended because it does not experience contact at start and shutdown. The hybrid system, of course, requires more axial length and must be designed to prevent the two bearings from fighting each other to avoid overload.

The primary types of rolling element bearings are listed in Table 6-6 along with advantages and disadvantages. Figures 6-66 and 6-67 show examples of such bearings. Various arrangements of duplex pairs of angular-contact ball bearings can be used, as shown in Fig. 6-68, to provide bidirectional axialload capability and overcome their main disadvantage. Springs are typically used to maintain axial preload of the bearings to remove internal clearance and control the radial stiffness. The springs also provide axial compliance to accommodate thermal effects. In order to provide roller guidance without end wear, some roller bearings are preloaded radially by negative internal clearance achieved through a thin-flexible outer race.

Rolling-element bearings are speed-limited due to parasitic loads from ball or roller inertia. This is typically expressed as a DN limit, defined as the product of the inner-race bore diameter $\mathrm{D}$ (in $\mathrm{mm}$ ) and the pump rotational speed $\mathrm{N}$ (in $\mathrm{rpm}$ ). A practical DN limit for Conrad-type and cylindrical roller bearings is 1.6 million, while angular-contact bearings have shown reliability up to 2.1 million. However, the life of the bearings at even lower DN values may be limited because of the lubrication available and the load combinations experienced. The higher DN values lead to higher centrifugal forces, higher sliding velocities, and increased fluid churning. The propellant will also affect the DN limit; e.g., the maximum values just cited have been achieved in liquid hydrogen, but liquid-oxygen bearings have not typically been used at these limits. Note that for a given transmitted horsepower, the shaft diameter based on allowable stress does not decrease proportionally with the increase of shaft speed. Thus, the required bearing DN value rapidly increases for high-speed turbopumps. As a result, the turbopump rpm may be limited by the bearing DN value.

Ball-bearing radial stiffness ranges from 0.2-1.5million $\mathrm{ft}^{-1}$ depending upon bearing size, contact angle, speed, mounting compliance, and applied load. As an example, the stiffness of a particular 45$\mathrm{mm}$ ball bearing with $750-\mathrm{lb}$ axial preload will be approximately $500,000 \mathrm{ft}^{-1}$ at $30,000 \mathrm{rpm}$ if the outer ring is allowed to tilt, but will be significantly stiffer if the outer ring is rigidly mounted. Roller-bearing stiffness is typically higher, ranging up to 2.0 -million $\mathrm{ft}^{-1}$. To achieve a given stiffness, bearing design is critical to the successful control of rotor-dynamic response.

During the turbopump design phase, computer programs are used to predict bearing characteristics and to optimize bearing geometry to meet design goals. Stiffness is calculated to support rotordynamic analysis of critical speeds and unbalance response. Ball excursions determine required cage pocket-clearance and structural criteria. Fatigue life, local stresses, and sliding-velocity calculations are used to assess the effects of variations in bearing geometry and operating conditions. Although the rolling bearing is a dynamic system, it is most often 


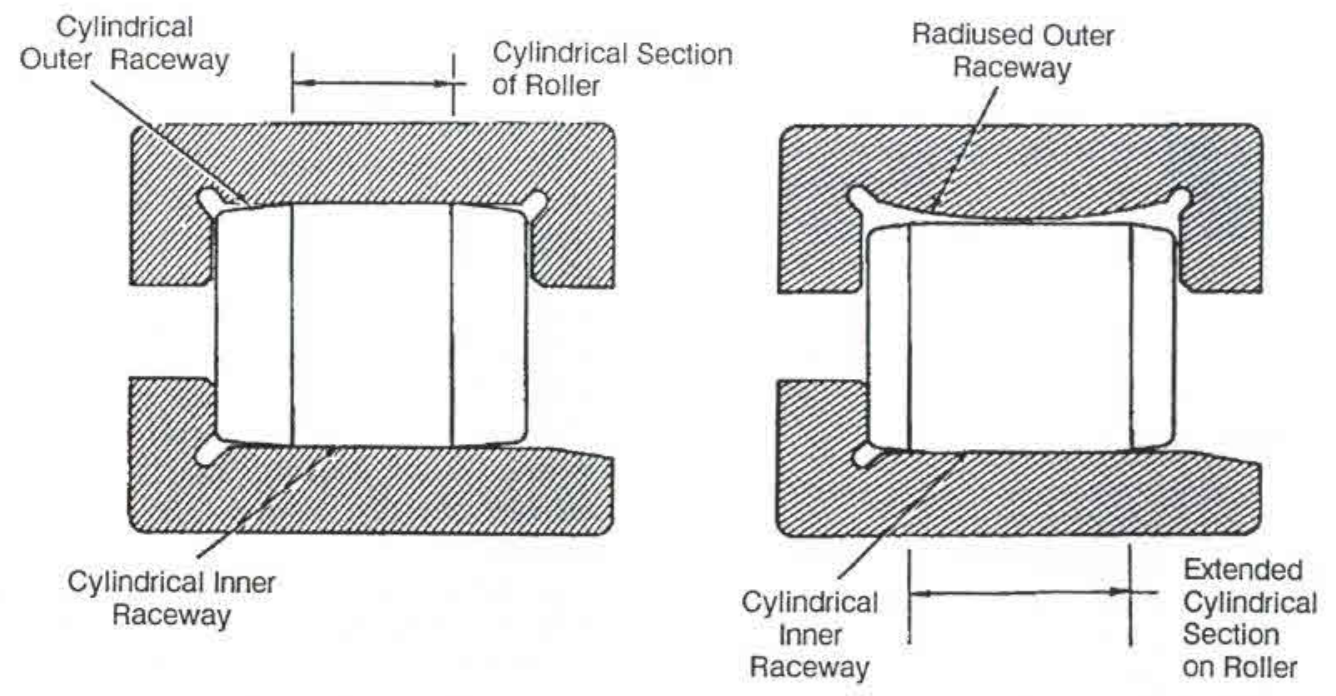

Fig. 6-67 Typical roller-bearing designs.

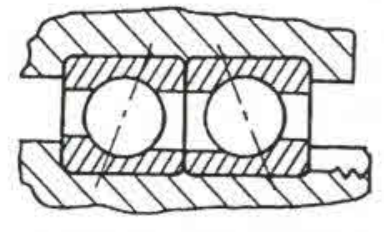

Duplex DB (back-to-back)

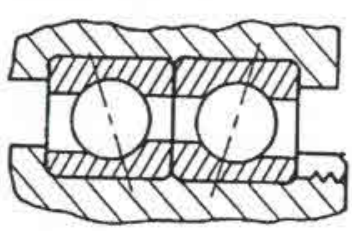

Duplex DF (face-to-face)
Fig. 6-68 Typical duplex-pair arrangements of ball bearings.

analyzed in a quasi-static manner; the forces and moments due to motion are determined for the assumed design configuration (speed, load, number and size of rolling elements, internal clearance, and material properties), and then the bearing-system equilibrium is established by treating the forces and moments as static quantities. These solutions are contained in commercially available computer codes. Several of the first were written by A.B. Jones, based upon his high-speed bearing analyses (Ref. 612). SHABERTH (SKF Technology Services, Ref. 613) includes options for automatically adjusting fits and clearances in response to temperature effects on shaft and bearing materials. The ADORE computer code by Pradeep Gupta (Ref. 6-14) performs a true dynamic analysis by solving the equations of motion for the interaction of the cage with the balls and rings, and can be used to assess the effects of cage clearances and mass and geometry on cage stability.

The axial loads generated by turbopump rotors at mainstage often exceed the load capacity of ball bearings, so the function of rotor axial-position control is performed by a balance piston operated by pump internal pressure. Ball bearings with axialtravel stops can limit axial motion during start and shutdown transients when loads are of short duration and within ball-bearing strength. Relatively compliant springs permit the balance piston to position the rotor without loading the ball bearings excessively.
The most commonly used material for bearing rings and balls, vacuum-melted 440-C steel, although not superior in either category, combines hardenability and corrosion resistance better than most other materials. Since short bearing life is a major constraint on engine utility, several alternative materials have been identified which may enhance life and reliability. These include CRB-7 and BG- 42 steels, which have higher hardness, and the ceramic silicon nitride $\left(\mathrm{Si}_{3} \mathrm{~N}_{4}\right)$, which is harder and $60 \%$ less dense than steel. In addition, the failure mode of the silicon nitride is generally fatigue-spalling rather than fracture. It thus appears to be a better material for the balls of a high-speed turbopump, but further demonstration of this is required. The most widely used cage material for propellant-cooled bearings is Armalon $^{\mathrm{TM}}$, a fiberglass-reinforced polytetrafluoroethylene (PTFE), or Teflon ${ }^{\mathrm{TM}}$. The glass fabric, impregnated with PTFE, gets mandrel-wrapped under heat and pressure into a tube of near net size, and then machined to final shape. Its advantages include light weight and high strength at cryogenic temperature, and resistance to ignition in $\mathrm{LO}_{2}$. Although the Teflon ${ }^{\mathrm{TM}}$ provides some friction reduction through film transfer, the glass fibers may promote wear. Friction-reducing treatments for Armalon $^{\mathrm{TM}}$ include phosphate-bonded $\mathrm{MoS}_{2}$ (which is quickly worn away) and a fused coating of fluorinated ethylene propylene (FEP), which has shown good improvement in bearing wear-life.

To maximize propellant-cooled-bearing life potential, a positive flow of propellant must be provided, and coolant temperature and pressure should be controlled to avoid vaporization of the fluid in the bearing. This may require maintaining high pressure in the bearing area by throttling flow downstream of the bearing. Coolant may be jet- or flood-fed, preferably to each bearing individually rather than in series. Particulate matter should be excluded by filter or traps to avoid bearing-surface damage.

A most important consideration in bearing design concerns the required operating life of the 
rocket engine. The bearings must have adequate statistical probability of exceeding this. One accepted life rating for rolling-element bearings, the "B-1 life," denotes the operating life in hours at which $1.0 \%$ of a set of bearings at a fixed load and speed will have failed by contact fatigue. B-1 life is often used to show life potential, but it is recognized that the calculation methodology evaluates the fatigue life under ideal lubrication. By contrast, the bearing environmental conditions in rocket-engine turbopumps frequently lead to wear rather than fatigue. Therefore, adequate B-1 life is necessary but not sufficient to assure meeting bearing-life needs.

Hydrostatic bearings offer an attractive means of overcoming limitations of rolling bearings, as they are theoretically speed-limited only by the centrifugal shaft stress, and thus can operate at journal speeds to approximately $5,000,000$ DN. Additional benefits include increased stiffness and load capacity (with sufficient fluid pressure and flow available) and significant damping, which is minimal with rolling bearings. Since external pressurization must develop the significant load capacity, hydrostatic bearings are subject to rubbing during start and shutdown. Means of avoiding wear during these periods include auxilliary rolling bearings, wearresistant materials, and "prepressurization" before start. Two types of radial hydrostatic bearings are used in turbopumps: orifice-compensated and axially-fed.

Figure 6-69 illustrates a recessed, orifice-compensated radial hydrostatic bearing. Its load capacity arises from fluid pressure responding to two flow restrictions in series. The pressure drop across the bearing has approximately equal steps: the first in the compensating orifice and the second in the close clearance between the journal and the lands defining the recess boundaries. A radial motion of the journal reduces the flow area on the side of the approach, increasing the local flow-restriction, causing the fluid pressure to rise in the associated recess. Simultaneously, the land flow restriction opposite is reduced, with a resultant decrease in the associated recess pressure. The relative pressure change, acting over the projected bearing area, provides a force tending to restore the journal to its original position.

Fluid forced through an annular space can generate significant stiffness across the radial gap. With the large pressures available in turbopumps, stiffnesses of over a million $\mathrm{lb} / \mathrm{in}$. can be produced. Converging clearance assures positive stiffness and enhances load capacity with minor increases in leakage flow.

Hydrostatic bearings differ from rolling bearings because the fluid film introduces significant damping and cross-coupling. The importance of these characteristics must be assessed based on rotor-dynamic response. High values of direct stiffness can be obtained by adjusting size, pressure available, and bearing geometry, somewhat independent of the fluid used, while the cross-coupling and damping generally increase with the fluid viscosity.

Hydrostatic bearings permit additional flexibility in selecting the basic turbopump configuration; the restrictions associated with the rolling-bearing DN and stiffness limits often result in a rotor configuration that operates between critical speeds and, therefore, has significant operating-speed restrictions to maintain safety margins. By increasing the shaft and bearing stiffness, subcritical designs with less

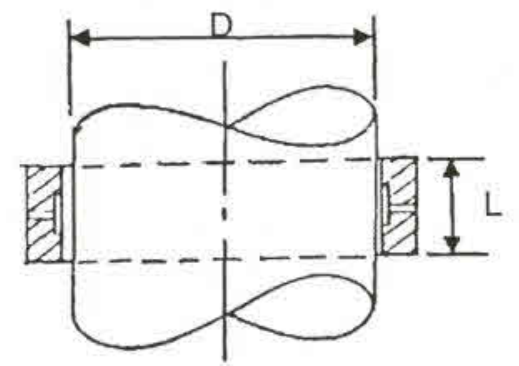

(B)

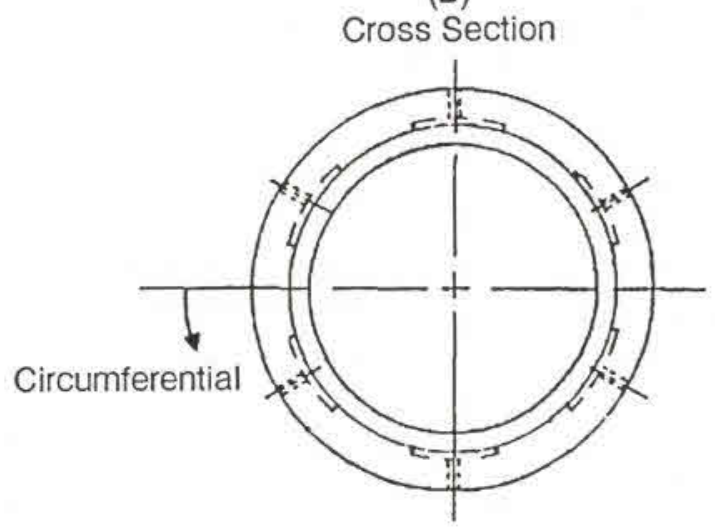

(A)

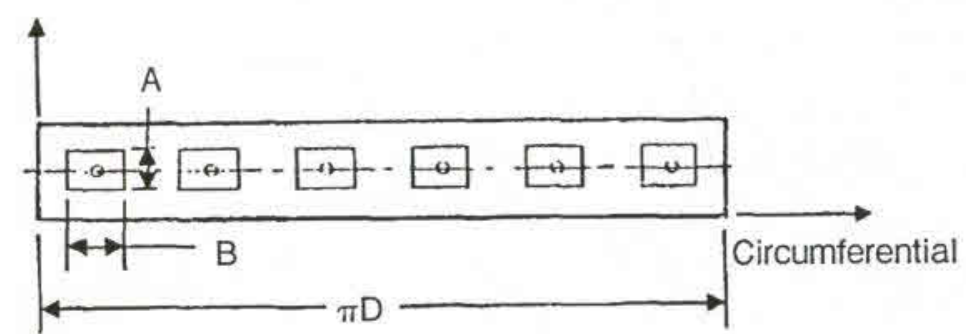

(C)

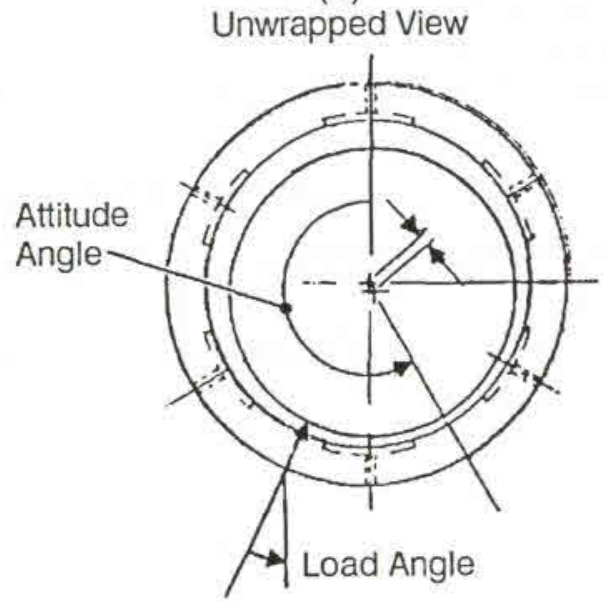

Axial View

Fig. 6-69 Typical hydrostatic-bearing features. 


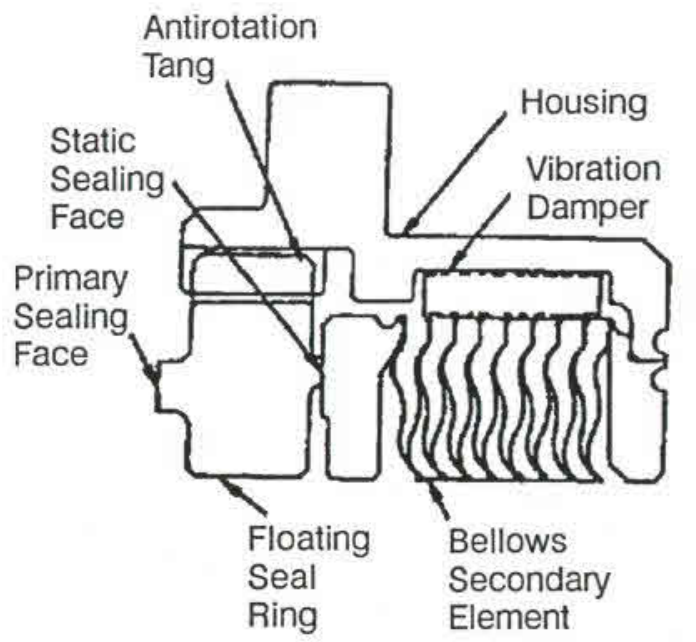

Face Contact Floating Seal Ring Bellows Secondary Element Seal

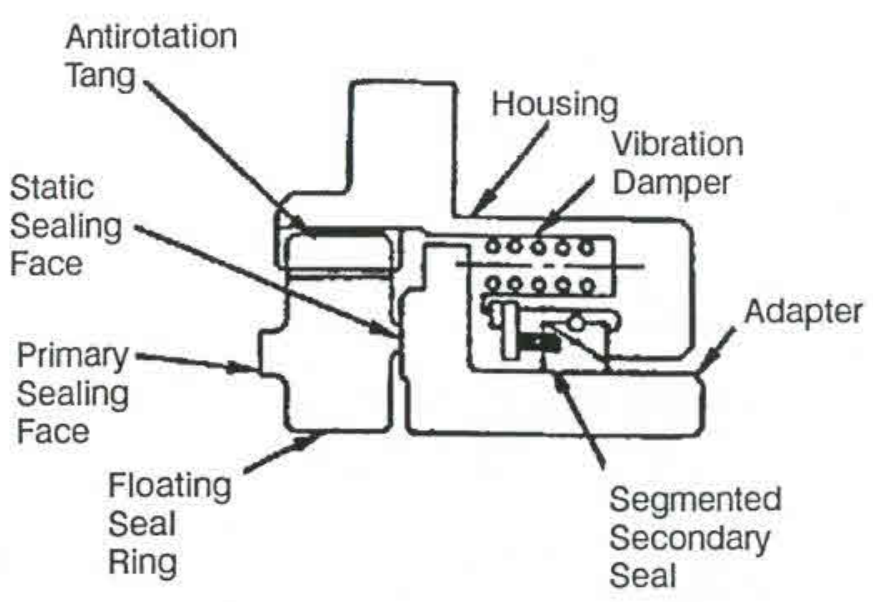

Face Contact Floating Seal Ring Segmented
Secondary Seal

Fig. 6-70 Face contact seals.

restriction of operating speeds are more attainable.

The approximate load capacity of an orificecompensated hydrostatic bearing is $25 \%$ of the product of the projected area (length $\mathrm{x}$ diameter) and the available pressure drop, and this rough rule may be used in preliminary size-selection. However, the minimum journal diameter will often be determined by other considerations, such as shaft strength and stiffness.

Bearing radial clearance has a significant effect upon the bearing stiffness, damping, crosscoupling, and leakage flow. As a general rule, there should be at least $0.001 \mathrm{in}$. of radial clearance per inch of journal radius. Sometimes a larger value is used for manufacturing economy, but the relative benefit of larger tolerances must be traded off against the consequent increase in leakage flow and lesser stiffness.

The hydrodynamic relations describing flow and pressures in a hydrostatic bearing are complex, and analyses are performed with computer programs specifically developed for this purpose. For orificecompensated bearings, analysis is generally based on Reynolds' equations with corrections applied to approximate the effects of high levels of turbulence. Improved codes are being developed that use the more basic Navier-Stokes relations. Analytic methods used for axially-fed hydrostatic bearings is based upon work initially performed to determine the characteristics of seals.

\section{Dynamic-Seal Design}

Turbopump dynamic seals have two main purposes: to prevent or minimize the leakage of propellants or fluids between the rotating components, and thus improve efficiency, and to prevent mixing of incompatible fluids, e.g., fuel and oxidizer, for reliable, safe operation. A secondary purpose has proven to be of value in high-speed turbopumps: ability of seals through proper design to provide significant damping and stiffness to the rotordynamic system. The seal system, i.e., the arrangement of seals, drains, and purges, must seal reliably at all extremes of operation and allow a single seal failure without destructive failure of the turbopump (Ref. 6-10).

The type of seal for each application will be selected to satisfy the pump operating conditions and the seal limitations. The seal system design and the seal performance limits are iterated in terms of pressure capability, temperature limits, speed limits, wear life, leakage, space requirements, and cost. The principal types of seal used in turbopumps are facecontact, segmented shaft-riding, floating-ring,

Table 6-7 Relative leakage of seals.

\begin{tabular}{|llcl|}
\hline $\begin{array}{c}\text { Type of } \\
\text { Seal }\end{array}$ & Leakage & $\begin{array}{c}\text { Typical } \\
\text { radial gap, in. }\end{array}$ & Fluid \\
\hline Face contact & Minimum & 0.0003 & Liquid/Gas \\
$\begin{array}{l}\text { Segmented shaft-riding } \\
\text { Hydrostatic/hydrodynamic }\end{array}$ & Low & 0.0005 & Gas \\
Floating ring & Low & $0.0003-0.0005$ & Liquid/Gas \\
Labyrinth & Medium & $0.002-0.003$ & Liquid/Gas \\
& High & $0.005-0.010$ & Liquid/Gas \\
\hline
\end{tabular}


Table 6-8 Face-contact-seal speed and PV limits for typical propellants.

\begin{tabular}{|lcc|}
\hline Fluid & $\begin{array}{c}\text { Max. Speed, } \\
\mathbf{f t} / \mathbf{s}\end{array}$ & $\begin{array}{l}\text { Max. PV, } \\
\text { psi X ft/s }\end{array}$ \\
\hline $\mathrm{LH}_{2}$ & 500 & 50,000 \\
$\mathrm{LOX}$ & 200 & 25,000 \\
$\mathrm{RP}-1$ & 300 & 25,000 \\
GH 2 & 400 & 20,000 \\
Hot gas & 200 & 10,000 \\
\hline
\end{tabular}

hydrostatic/hydrodynamic, and labyrinth. Table 6-7 gives a relative comparison of their effectiveness.

The face-contact seal (Fig. 6-70) provides the lowest leakage and generally will be used when the pressure-speed-life limits are consistent with reliable operation. The sealing comes through rubbing contact between a rotating mating ring attached to the shaft and the seal face. The mating ring is generally plated with a wear-resistant hard plating and is lapped flat. The seal ring is generally a selflubricating carbon material with a flat sealing face. The seal ring can be floating to minimize thermal distortions or can be press-fitted into a metal adapter. The seal ring is loaded against the mating ring with either a welded-metal-bellows secondary element or compression springs. The sealing dam is established to balance the pressure-induced closing forces to maintain a satisfactory face load at high pressure. The secondary sealing element can be a welded bellows, piston ring, segmented ring, or elastomer, depending on the operating requirements. Welded metal bellows are ordinarily used for temperatures below -65 or above $500^{\circ} \mathrm{F}$.

Face-contact seals are speed-limited by heat generation at the rubbing face and cooling capacity of the environment. The heat generation rate is a function of the contact load and velocity of the rubbing surface. The limit is given as a PV factor, where $\mathrm{P}$ is the unit contact load in psi and $\mathrm{V}$ is the rubbing velocity in $\mathrm{ft} / \mathrm{s}$. The PV limit is based on the cooling capacity of the environment. Table 6-8 gives PV limit for typical propellants. Clearancetype seals, such as labyrinth, floating-ring, or hydrostatic/hydro-dynamic, are used for higher speeds.

The segmented shaft-riding seal (Fig. 6-71) consists of segmented sealing rings that are loaded against the shaft with a garter spring and the pressure differential to form a radial dynamic seal. An axial static seal is formed by loading the segments against a flat surface on the seal housing using springs and the pressure differential. The segmented rings may consist of three rings with overlapping joints or single rings with tongue and groove joints. Having the segmented rings in rubbing contact minimizes leakage. The segments are loaded by the pressure differential and are generally. limited to approximately 100 psi.

The floating-ring seal (Fig. 6-72) consists of a solid sealing ring that is free to float in the radial direction for minimum operating clearance. The radial dynamic seal is formed by the close clearance to the shaft; the axial static seal, by loading the ring against the seal housing. The ring is partially pressure balanced in the axial direction to control the axial load and the radial friction force. The centering force is provided by the pressure profile in the clearance gap. The force increases on the side approaching contact and decreases on the opposite side to give a centering force when the ring moves off center relative to the shaft. The centering force is increased by a factor of approximately 3 when a convergent taper is utilized on the seal-ring bore. The optimum taper ratio of inlet clearance to exit clearance is approximately 1.5 to 2.0 for most fluids.

Hydrostatic and hydrodynamic geometry may be utilized on face seals, segmented seals, and floating-ring seals to provide a lift force proportional to the operating gap at the sealing surface. The lift force increases as the sealing surface approaches rubbing contact and decreases for larger gaps to form a very small $(0.0005$-in.) self-adjusting

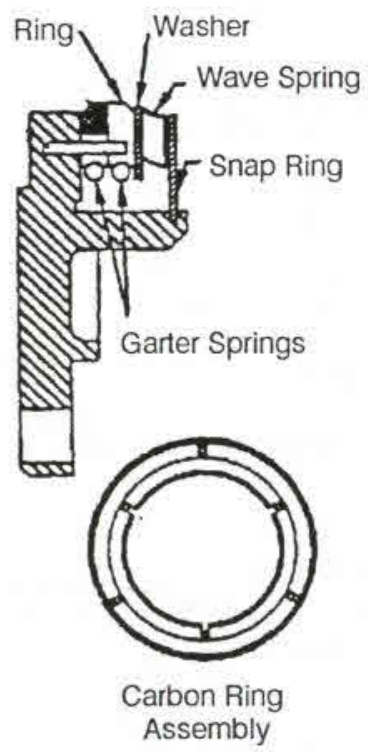

Segmented 3-Ring Design with Overlapping Joints
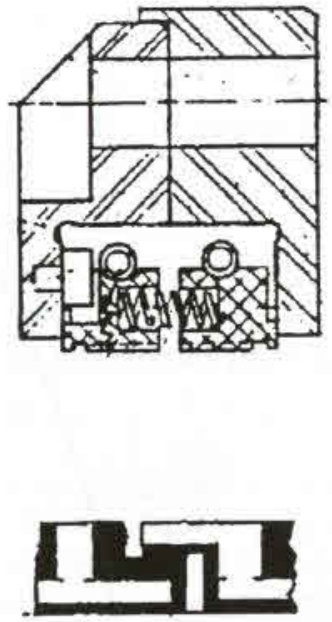

Segmented Single Ring Design With Tongue and Groove Joints
Fig. 6-71 Segmented shaft-riding seals.
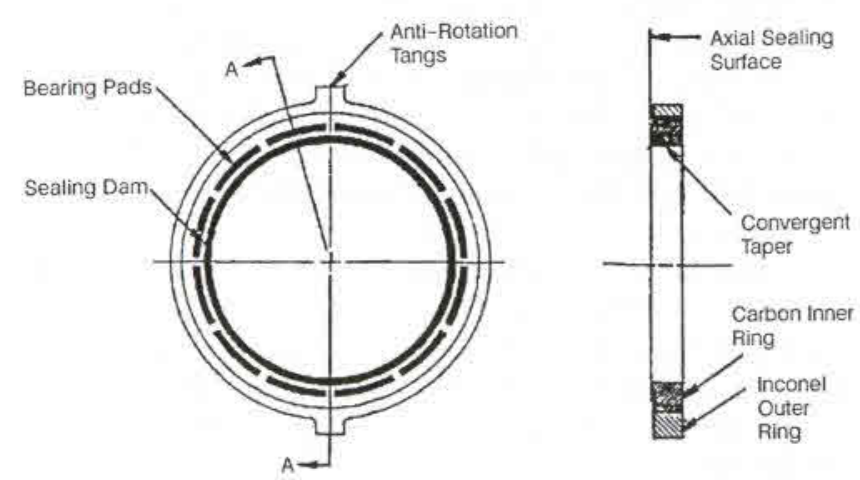

Fig. 6-72 Floating-ring seal. 


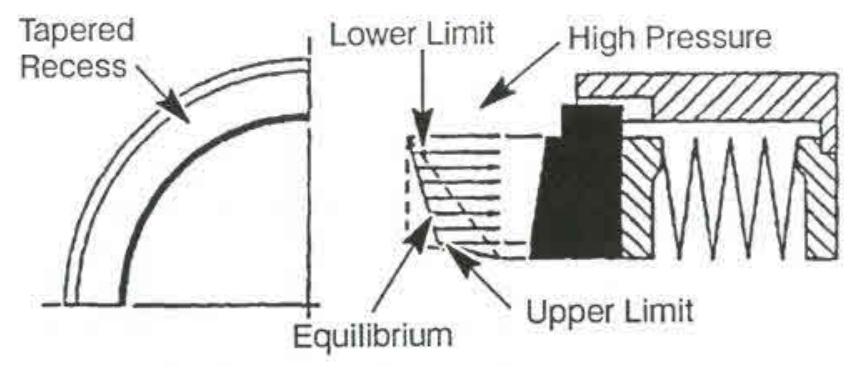

Fig. 6-73 Convergent tapered-face hydrostatic face seal.

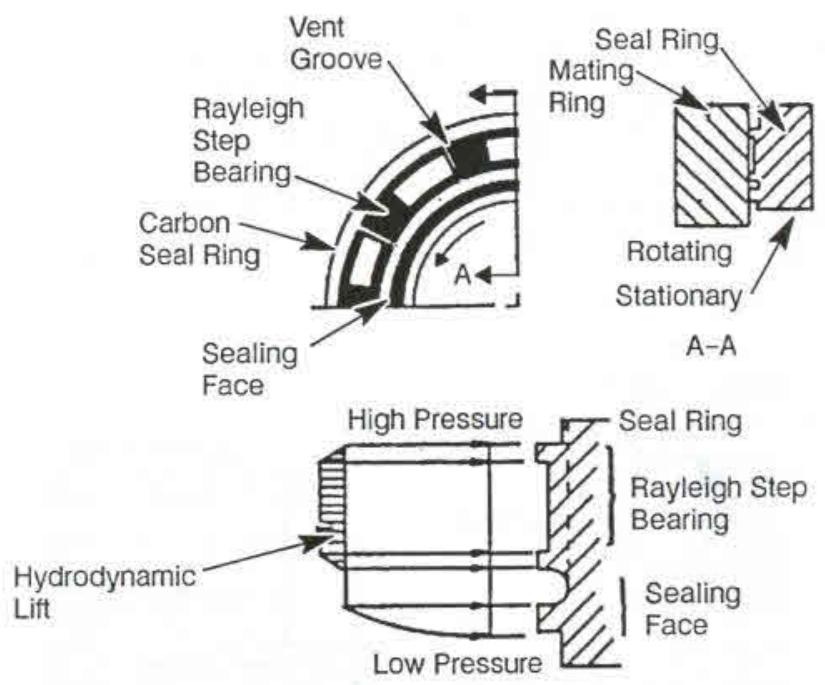

Pressure Profile

Fig. 6-74 Rayleigh-step hydrodynamic face seal.

clearance yielding minimum leakage and no steadystate rubbing contact. A convergent tapered face (Fig, 6-73) may be used to provide hydrostatic lift. Hydrodynamic lift may be provided by Rayleigh step pads (Fig. 6-74) or spiral grooves (Fig. 6-75). The Rayleigh step pads provide hydrodynamic lift by viscous pumping of the sealed fluid in the recessed pads. The spiral grooves develop hydrodynamic lift by viscous pumping the sealed fluid in the spiral grooves. Both concepts are pressurebalanced by locating the sealing dam inside of the hydrodynamic geometry. Both the hydrostatic and hydrodynamic-lift seals, as successfully tested in the laboratory, have demonstrated positive control of the quality of the sealed fluid. To date, these seals have not been used in flight turbopumps and are not as reliable if the fluid is susceptible to vaporization in the seal.

The labyrinth seal (Fig. 6-76) can be used when low cost and reliability are the primary consideration or when the pressure and speed limits of other types of seal are exceeded. Typically, the wear rings on the rotating impellers are labyrinth seals because of the higher velocity due to the required diameter. Since the clearance gap must be large enough to allow for mislocation tolerances and shaft
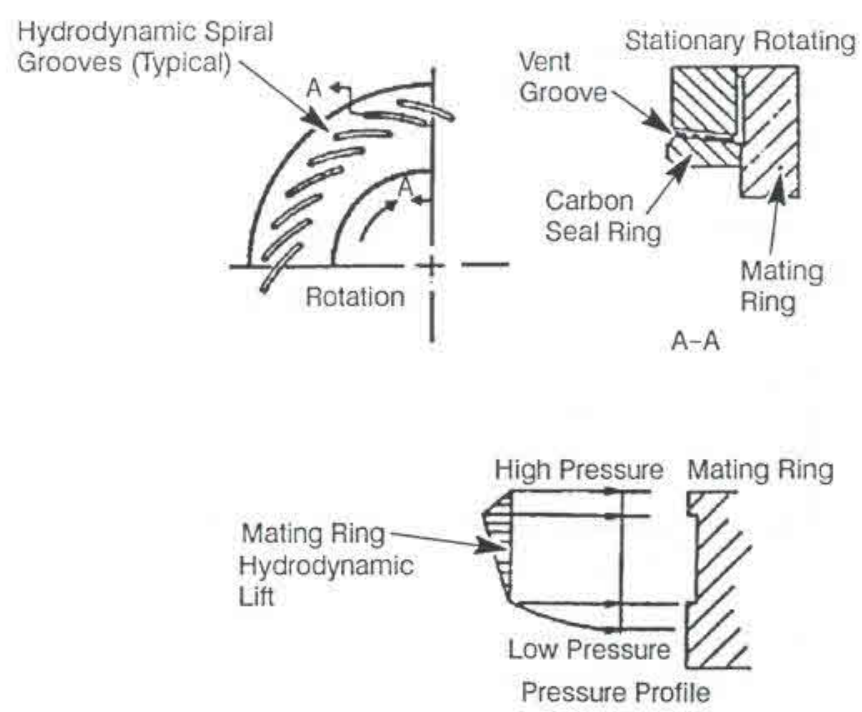

Fig. 6-75 Spiral-groove hydrodynamic face seal.

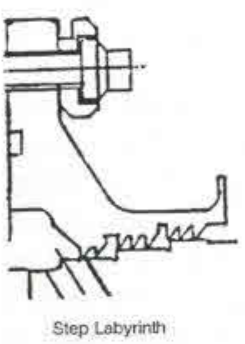

Fig. 6-76 Labyrinth-seal designs.

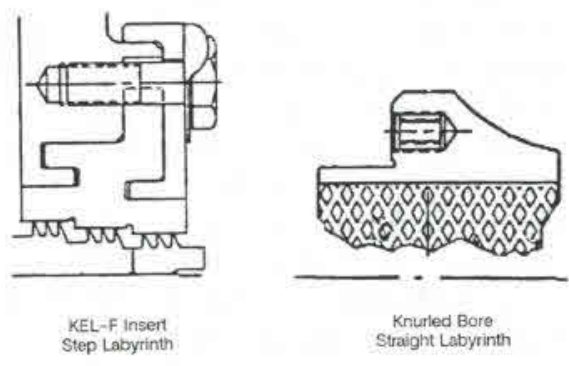

radial motion, the leakage is significantly higher than for the other types. Step-labyrinth seals are used to reduce the leakage by creating barriers to reduce the velocity head. The number of teeth, tooth pitch, and height will be optimized to give minimum leakage. Figure 6-77 shows typical data on the effectiveness of the labyrinth design for reducing leakage with a liquid propellant. Kel-F plastic inserts are used for oxidizer seals to allow rubbing contact without the hazard of ignition. A straight or convergent labyrinth with a knurled bore and smooth shaft provides additional dynamic stability by reducing the circumferential velocity relative to the axialleakage velocity.

Table 6-9 gives pressure and temperature limitations for the principal types of seal. The seal type will be selected to satisfy the pressure-speed-life limits with minimum leakage and maximum reliability.

Oxidizer and fuel on a rotating shaft will usually be separated by a primary oxidizer seal, an inert-gaspurged intermediate seal, and a fuel seal with separate drains for the oxidizer and fuel. The purged intermediate seal provides a pressure barrier between the drain cavities to prevent mixing of the propellants. For reliable operation, high-pressure propellants may require staged sealing to reduce the pressure at the intermediate seal. Figure 6-78 shows a representative seal system incorporating these 

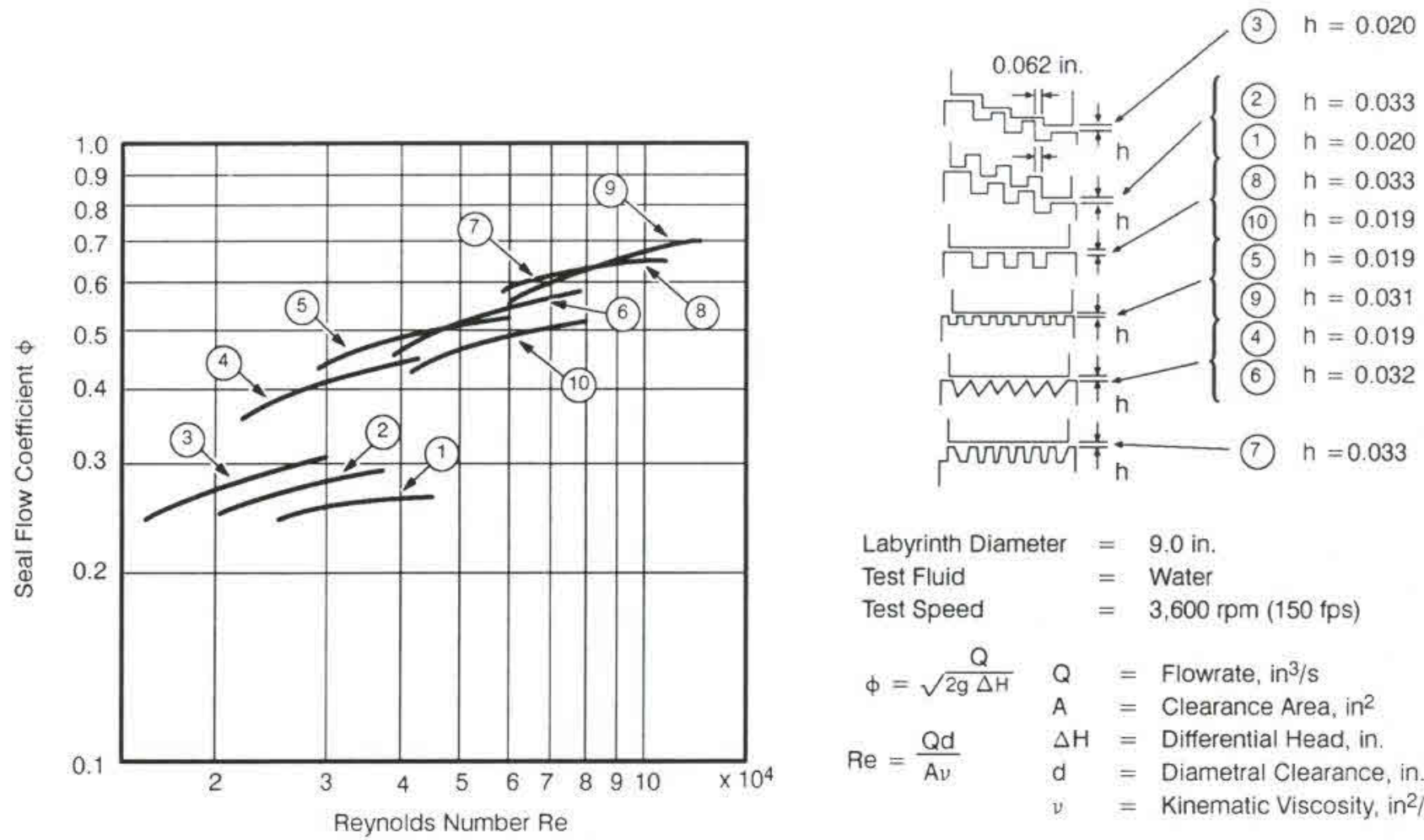

$$
\begin{array}{ll}
\text { Labyrinth Diameter } & =9.0 \mathrm{in} . \\
\text { Test Fluid } & =\text { Water } \\
\text { Test Speed } & =3,600 \mathrm{rpm}(150 \mathrm{fps})
\end{array}
$$

$$
\begin{aligned}
\phi=\sqrt{2 \mathrm{~g} \Delta H} & \mathrm{Q}=\text { Flowrate, in } 3 / \mathrm{s} \\
\mathrm{A} & =\text { Clearance Area, in }{ }^{2} \\
\mathrm{Re}=\frac{\mathrm{Qd}}{\mathrm{Av}} & \mathrm{\Delta H}=\text { Differential Head, in. } \\
& \mathrm{d}=\text { Diametral Clearance, in. } \\
v & =\text { Kinematic Viscosity, in } 2 / \mathrm{s}
\end{aligned}
$$

\begin{tabular}{|c|c|c|c|c|}
\hline \multirow{2}{*}{ Seal Type } & \multirow{2}{*}{$\begin{array}{l}\text { Recommended } \\
\text { Max Pressure } \\
\text { (psig) }\end{array}$} & \multicolumn{2}{|c|}{$\begin{array}{l}\text { Temperature } \\
\text { Limit ('A }\end{array}$} & \multirow{2}{*}{ Remarks } \\
\hline & & Min & Max & \\
\hline $\begin{array}{l}\text { Face Contact } \\
\text { Metal bellows } \\
\text { Piston ring } \\
\text { Elastomeric }\end{array}$ & $\begin{array}{r}500 \\
750 \\
1,000\end{array}$ & $\begin{array}{r}.423 \\
.423 \\
.65\end{array}$ & $\begin{array}{r}1500 \\
1500 \\
500\end{array}$ & $\begin{array}{l}\text { Cryogenic or reactive fluids } \\
\text { Hot gas or cryogenic } \\
\text { Lubricated }\end{array}$ \\
\hline $\begin{array}{l}\text { Circumferential } \\
\text { Segmented } \\
\text { Floating ring } \\
\text { Labyrinth }\end{array}$ & $\begin{array}{l}100 \\
5,000 \\
\text { Unlimited }\end{array}$ & $\begin{array}{l}.423 \\
.423 \\
.423\end{array}$ & $\begin{array}{l}1000 \\
1200 \\
1800\end{array}$ & $\begin{array}{l}\text { Hot gas and purge gas } \\
\text { Hot or purge gas, long life } \\
\text { All fluids, reliable }\end{array}$ \\
\hline Hydrostatichydrodynamic & 500 & & & \\
\hline
\end{tabular}

Fig. 6-77 Effect of labyrinth design on leakage.

Table 6-9 Seal pressure and temperature limits.

concepts. The leakage drains are sized to accommodate the maximum leakage while maintaining a pressure at the intermediate-seal discharge that does not exceed the purge pressure between the intermediate-seal rings. This assures a positive separation of the fluids at all times.

Some applications require a positive seal during prestart conditioning but an open seal during operation. For such cases, static liftoff seals are typically used that close by spring forces until internal pressures increase with rotation to provide a force large enough to completely open the seal. Typically, the seal will open at a shaft speed of 20 $30 \%$ of the mainstage speed.

\section{Turbopump Gear Design}

Gear drives for rocket-engine turbopumps are used to achieve maximum efficiency in low-to-medium

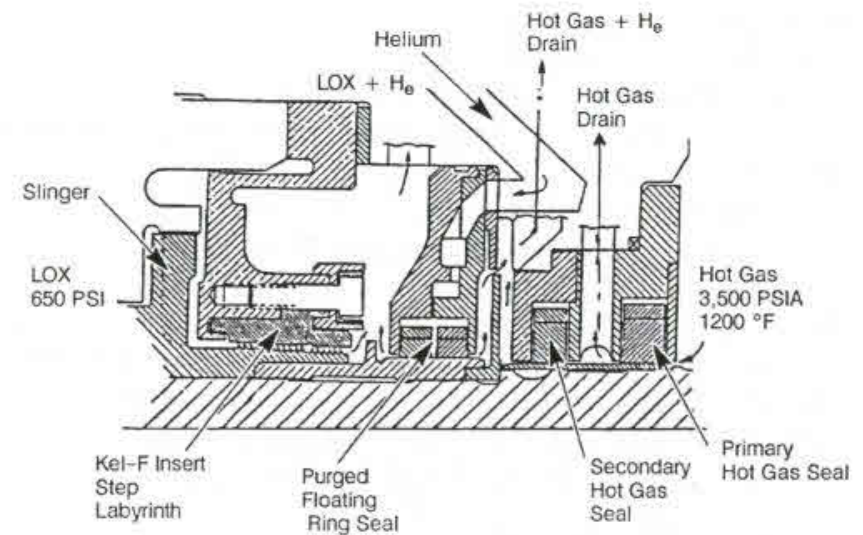

Fig. 6-78 Typical seal system for separating high-pressure propellants.

power applications $(100-5000 \mathrm{hp})$. The need to operate the rocket-engine pumps at low inletpressure frequently limits the allowable operational pump speeds. In general, the optimum turbine speed will differ from that of the pumps; and if there are significant differences in oxidizer and fuel density, it is desirable to operate the pumps at different speeds as well. Geared systems provide a means of operating all components at optimum speed (Ref. 6-11).

Geared turbopumps find application in gasgenerator cycles for both storable and cryogenic propellants because of the high turbine speed required for the high pressure ratio and low mass flow. Upper-stage liquid-hydrogen-fueled engines are also 
candidates because the large difference in density between fuel and oxidizer causes different optimum pump-speeds, and the low power level results in a geared pump being lighter than separate, directdriven fuel and oxidizer pumps.

Several major U.S. launch vehicles (Atlas, Delta, and Titan) utilize geared turbopumps for boost stages of flight, as does Japan's N-2, which is similar to the Delta. The European Ariane and the U.S. Centaur use a gear-driven LOX-LH 2 turbopump.

Lubrication. The method used for lubrication and cooling has a fundamental influence on the design of the gear system. Lubrication schemes are often categorized by use of oil or propellant, but the design will be most influenced by the degree of good or poor lubrication.

For small $\mathrm{LOX} / \mathrm{LH}_{2}$ pumps (the Centaur RL10, for example), gaseous hydrogen can be used to cool the gears and bearings with lubrication supplied by dry film coatings, such as molybdenum disulfide, applied to contacting surfaces. Such coating gives poor lubrication, but allows a workable design by keeping contact loads and speeds low. Design emphasizes minimize wear.

Higher-power applications (typically above several hundred HP) demand good lubrication. This generally means oil; but, with RP-1 (kerosene)-fueled engines, equal success has been obtained using kerosene with an extreme pressure additive (EPA) to minimize scoring of the gear. With good lubrication, turbopump gear design follows conventional practice, but gives special emphasis to factors significant at high pitchline speeds and tooth loadings.

Although fluid lubricating properties are important, flowrates and method of application are dictated by cooling requirements. Frictional heating in the gear mesh takes roughly $0.5 \%$ of the power transmitted. As pitchline velocity increases, tooth cooling becomes critical. Directing jets of lubricant at the disengaging side of the mesh, where the working flanks of the teeth are first exposed, provides efficient cooling if high jet velocities (velocities at least as large as the pitchline velocity) can overcome windage flows.

Both recirculating and single-pass oil systems are being used on larger turbopumps. The lubricant systems must be capable of meeting both gear and bearing flow-requirements. Inert-gas purges may also be used to control gearbox pressure in order to reduce lubricant foaming and to improve drainage or oil scavenging.

Gearbox arrangement. Figure 6-16 shows a cross section of the turbopump used on the Delta first-stage engine. It illustrates several factors that influence the overall turbopump and gear-train layout. The pumps and turbine are usually significantly larger than the gears and must interface with large fluid ducts. Therefore, packaging requirements usually force the impellers or turbine wheels to overhang the end of the shafts. It is often necessary to use idler gears or multiple reductions (even when the required speed reduction ratio could be obtained with a single mesh) to obtain sufficient spacing between pump and turbine shafts.
Gear alignment (shaft parallelism) is critical to obtaining maximum gear capacity. Highly loaded gears should, therefore, be centrally mounted between two bearings to minimize the deflection caused by the large transverse loads arising in the mesh. Gear alignment as well as the need to isolate the hot turbine housing and cold pump housing (in the case of cryogenic propellant) usually requires the gearcase structure be isolated from pump and turbine structures.

Rotor-dynamic and shaft bending limit the mass of discs, particularly on a high-speed shaft. To avoid this constraint, the turbine may be supported by its own set of bearings and linked to the high-speed pinion by means of a quill shaft that will accommodate misalignment and isolates loads.

Gear geometry. The factors that differentiate turbopump power gears from other high-speed aerospace power gears are higher tooth-face loads (tangential force per unit tooth width) and short life. Tooth loads may be as high as $5000 \mathrm{lb} / \mathrm{in}$. for a turbopump power gear, but life requirements are seldom longer than a few hours. These factors, along with the need for minimum weight and extremely high reliability, determine the design of the gears.

Involute spur gears have been used exclusively because of their high efficiency and simplicity. Although helical gears offer advantages by somewhat higher load capacity and smoother operation, they introduce axial loads that complicate bearing and casing design, and therefore have been avoided.

The critical concerns in gear performance are tooth-bending fatigue life, pitting fatigue life, and scoring resistance. Gear design must balance the sometimes competing parameters that affect these concerns to achieve the desired life.

Tooth bending strength is related to tooth size (diametral pitch), pressure angle, number of teeth, and face width. Face width is generally limited to 0.5 to 0.7 times the pitch diameter of the pinion (smaller gear) because unavoidable misalignment tends to load the tooth ends as width increases. Relatively high pressure angles (angle between a normal-to-the-tooth surface and the tangent-to-thepitch circle), typically $25 \mathrm{deg}$, are used because they allow a wider tooth base for strength and a larger radius of curvature at the point of contact to reduce hertzian contact stress.

Tooth size and number of teeth are related to the gear diameter. Smaller diameters reduce pitchline velocity and weight at the expense of higher tooth-loads. For a given pitch diameter, the larger the teeth, the smaller the contact ratio (i.e., the average number of teeth in contact at one time). High contact ratios give smoother operation (for reduced dynamic loads, especially at high pitchline velocities) and lower contact loads at the tooth tips where sliding velocity is a maximum. Contact ratio must be traded against tooth size (larger teeth provide greater bending strength). A contact ratio of 1.5 has proven near-optimum for turbopumps.

Standard tooth proportions can be modified to improve gear performance further. Pinions are frequently made with long addendums and gears made with short addendums to reduce sliding at the 
pinion tip, where wear tendencies are greatest, and to strengthen the pinion, which experiences a larger number of stress cycles than the gear. Pinion teeth can be further strengthened by increasing thickness at the expense of the mating gear. The involute profile may be relieved near the tip and root to account for large elastic deflections of teeth as they enter and leave the mesh. Crowning or end-easing is also employed to prevent maximum loads from occurring at the tooth ends.

Gear materials, processing, and quality. Materials, processing, and quality control are as essential as the basic design and geometry in meeting life requirements. For highly loaded power gears, life is generally limited by bending fatigue and wear. Deep-carburized, case-hardened steel provides high surface hardness (58-62 Rc) to gain wear resistance and fatigue life. Vacuum-melted and forged materials can further improve fatigue properties.

Tight control of tooth-form tolerances proves critical for minimizing dynamic loads from tooth spacing errors and ensuring uniform load distribution across the width of the teeth. To obtain the desired tooth-form accuracy without introducing detrimental machining effects in the critically stressed fillet region at the base of the teeth, the following process may be used:

- Teeth are rough-cut before heat-treatment, leaving a slight undercut below the working-flank surface.

- After heat treatment, the root area may be shotpeened to improve fatigue resistance further.

- Working flanks are finish-ground, taking particular care to avoid grinding in the fillet and root.

\subsection{DESIGN LAYOUT OF TURBOPUMP ASSEMBLIES}

Figure 6-79 presents the design layout of the A-1 Stage engine turbopump assembly. Logical packaging and arranging of the basic mechanical elements of the turbopump are among the considerations in preparing the layout. For instance, ease of development will be one of the more important criteria that influences the selection or arranging of the turbopump mechanical elements. Standard or proven mechanical detail should be extensively adopted in the layouts. Important turbopump designlayout considerations include these:

- Compatibility with engine systems packaging and plumbing

- Structural integrity

- Material selection (e.g., for weight and fluid compatibility)

- Control of system critical speeds and dynamic response

- Control of axial and radial loads on the rotating members

- Positive sealing of fluid mediums

- Control of fits and deflections

- Compensation for thermal expansion and contraction

- Ease of development

- Ease of assembly

- Ease of manufacturing

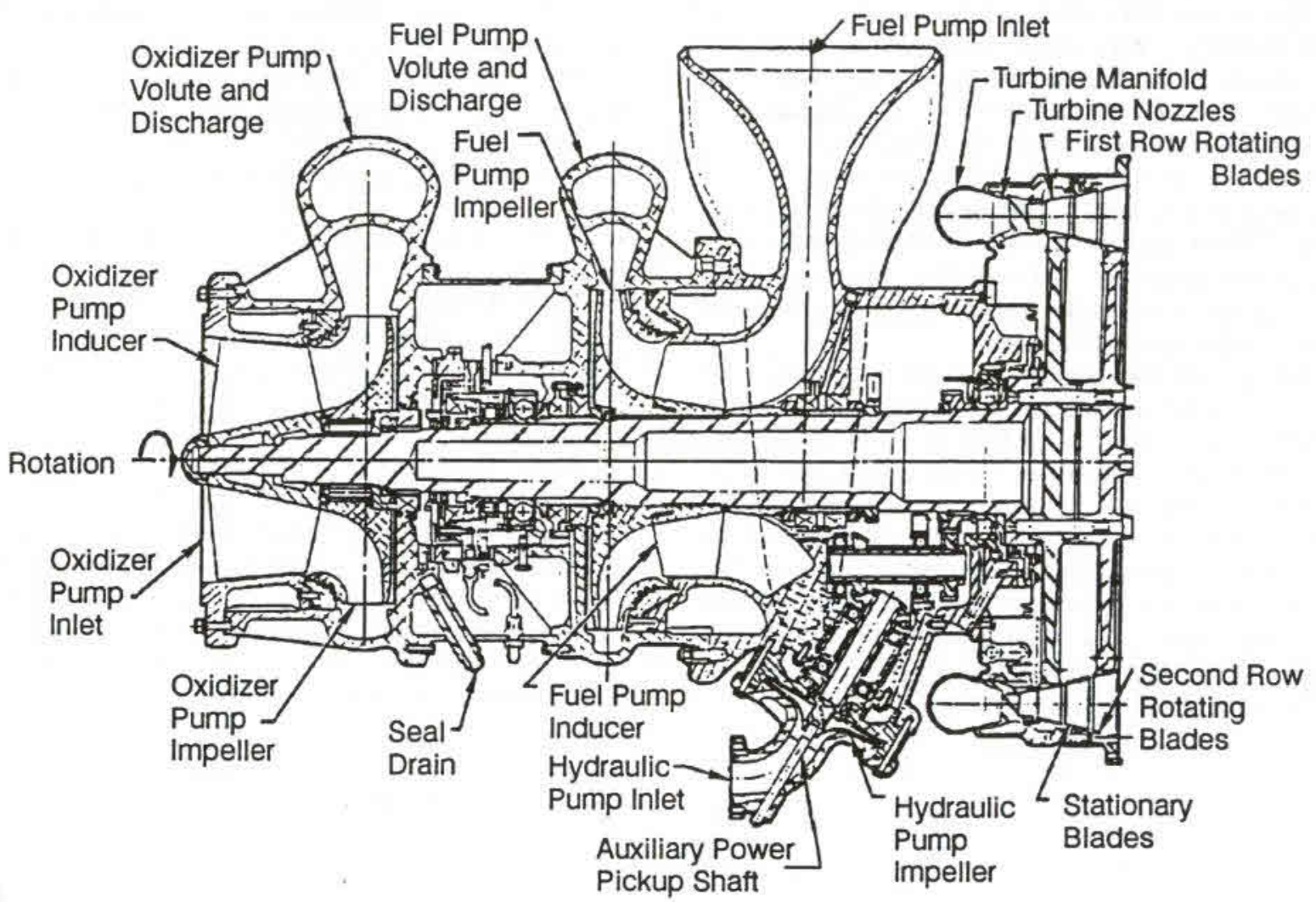

Fig. 6-79 Assembly design layout of the hypothetical A-1 Stage engine turbopump. 
Turbopump design layout demands considerable experience and skill. To illustrate the influence of some of these parameters, consider the SSME HPFTP of Fig. 6-5. The use of a scroll inlet to the first stage was driven by an engine system arrangement that would not permit an axial inlet. Also, the way the turbopump was mounted to the hot-gas manifold and the structural backbone of the engine led to a decision to limit the turbine-pitchline diameter, thereby giving up several points of turbine efficiency to reduce weight and provide better structural margin. In selecting materials for the turbine disks, no material was available that provided the structural integrity at the temperature of the hot-gas turbine drive fluid. As a result, the turbine disks had to be cooled with liquid hydrogen. However, this created an incompatibility; the hydrogen embrittled the disk material. Gold-plating the disks eliminated this problem.

One of the key ways to control the critical speeds is through the selection of the bearing locations. For the SSME pump in Fig. 6-5, the bearings were placed outboard, but this was driven by bearing DN limits that prevented placing the bearings over the part of the shaft that was carrying the torque. At the outboard locations the shaft diameter can be reduced because there is no shaft torque. To improve the dynamic response with these bearing locations, the pump must operate between the second and third critical-speed, and the seals must be designed to provide additional stiffness and damping. The ball bearing at the centerline of the shaft at the pump end was added after the original design to serve as a transient axial-thrust-control device (rather than using rubbing seal surfaces within the pump). This is a clutching ball bearing that carries the loads until the balance-piston pressure can take control. This usually results in the bearing being engaged until the pump speed reaches approximately $7000 \mathrm{rpm}$. Another favorable design feature of this pump is that every external seal is only required to seal the pump-inlet-pressure, which is close to $300 \mathrm{psi}$, rather than sealing the discharge pressure of $7000 \mathrm{psi}$. This was achieved by providing internal piston-ring seals to seal the high pressures and venting the area under the external casing back to the inlet pressure of the pump.

In laying out the pump, it is necessary to plan the complete turbopump for assembly and, as well, disassembly with a minimum loss of parts. However, the fits required sometimes dictate that parts be assembled by selective heating/cooling of adjacent parts that prevents disassembly without destruction of one of the parts. Another consideration in the design layout is the requirement for instrumentation and/or inspection. For example, it is frequently desirable to be able to inspect bearings between tests, but it is hard to do this without taking the turbopump off the engine. With duplex bearing pairs, some of the bearings are generally not inspectable without turbopump disassembly. Future turbopumps will likely rely more heavily on advanced instrumentation to measure bearing condition, rather than visual inspection.

The decision to design for ease of fabrication will depend on the relative importance of other parameters. For example, in the SSME pump (Fig. 65) welds were used extensively rather than bolted joints, even though this significantly increased the cost and difficulty of fabrication. This was done because in the design phase of this turbopump the emphasis was on weight and performance to assure meeting all projected mission requirements.

\subsection{REFERENCES}

6-1. "Turbopump Systems for Liquid Rocket Engines," NASA Monograph SP-8107, Aug 1974.

6-2. "Liquid Rocket Engine Turbopump Inducers," NASA Monograph SP-8052, May 1971.

6-3. Stripling, L. B., and Acosta, A. J., "Cavitation in Turbopumps - Part 1," Journal of Basic Engineering, Vol. 84, Sep 1962, pp. 326-338.

6-4. Stripling, L. B., "Cavitation in Turbopumps - Part 2," Journal of Basic Engineering, Vol. 84, Sep 1962, pp. 339-350.

6-5. Furst, R., and Desclaux, J., "A Simple Procedure For Prediction of NPSH Required By Inducers,"ASME Pumping Machinery - 1989. FED Vol. 81, July 1989 .

6-6. "Liquid Rocket Engine Centrifugal Flow Turbopumps," NASA Monograph SP-8109, Dec 1973

6-7. "Liquid Rocket Engine Axial Flow Turbopumps," NASA Monograph SP-8125, April 1978.

6-8. "Liquid Rocket Engine Turbines," NASA Monograph SP-8110, Jan 1974.

6-9. "Liquid Rocket Engine Turbopump Bearings," NASA Monograph SP-8048, Mar 1971.

6-10. "Liquid Rocket Engine Turbopump Rotating-Shaft Seals," NASA Monograph SP-8121, Feb 1978.

6-11. "Liquid Rocket Engine Turbopump Gears," NASA Monograph SP-8100, Mar 1974.

6-12. Jones, A. B., "A General Theory for Elastically Constrained Ball and Radial Roller Bearings Under Arbitrary Load and Speed Conditions," Journal of Basic Engineering, Jun 1960, pp. 309-320.

6-13. Hadden, G. B., Kleckner, R. J., Ragen, M. A., and Sheynin, L., "Steady State and Transient Thermal Analysis of a Shaft Bearing System Including Ball, Cylindrical and Tapered Roller Bearings," NASA CR No. 165365 (SKF Rept. No. AT810040) submitted to NASA Lewis Research Center, May 1981.

6-14. Gupta, P. K., Advanced Dynamics of Rolling Elements, Springer-Verlag, New York, 1984. 